\title{
Field reconstructions and range tests for acoustics and electromagnetics in homogeneous and layered media
}

\author{
Dissertation \\ zur Erlangung des Doktorgrades \\ der Mathematisch-Naturwissenschaftlichen Fakultäten \\ der Georg-August-Universität zu Göttingen
}

vorgelegt von

Jochen Schulz

aus Hannover

Göttingen 2007 
D7

Referent: ................... Prof. Dr. Roland Potthast Korreferent: ....................... Prof. Dr. Rainer Kreß Tag der mündlichen Prüfung: ......... 4ter Dezember 2007 


\section{Acknowledgements}

First of all, i want to thank my advisor Prof. Dr. Roland Potthast for giving me the opportunity of writing a thesis in the mathematical faculty as this is somewhat unusual and challenging for a physicist, which i am, or should i say was ? He tried to make the challenge of learning all the mathematics i missed a little bit easier and encouraged me to make all the small steps needed to accomplish this. Thoughout the time he acquired interesting projects and initiated activities going beyond the work on the thesis, which made the time much more interesting and joyful. I also want to thank him for beeing more than an advisor and occasionally sharing relaxing hours after the work. Furthermore, my thanks go to my second advisor Prof. Dr. Rainer Kreß for very helpful discussions and tips in the last months, as well as for contributing a substantial part of the work for a paper about mine detection.

Over the years of my thesis Prof. Potthast and Prof. Kress ensured my financial support, for which i am very grateful. In particular the financial support of the Fachhochschule Hildesheim/Holzminden/Göttingen, the state of lower saxony through funding the junior research group "Neue numerische Verfahren zur Lösung inverser Probleme" and the German Federal Ministry of Education and Research (BMBF) through funding the network "Metal detectors for Humanitarian Demining" is gratefully acknowledged.

I would like to thank all the technical and administrative staff of the institute for providing the environment and basis to make effective work possible.

Thanks go to my formerly room mate Klaus Erhard, who shared some of the work and joy and had encouraging words for me. I thank my room mate and friend Eric Heinemeyer for beeing always helpful, nice and sharing the many hours of, more or sometimes less, philosophical discussions while having tea or wine. Further, he introduced me to many music which can be heard while working and is inspiring all along. He also proof-read parts of my thesis, for what $i$ am very grateful.

Last, but not least, i want to express my deepest gratitude for my girlfriend Susanne Moysich for sharing good and bad times, as well as supporting and strongly believing in me over the years while i was writing this thesis. Further, i want to thank my family for always lending a ear for my problems while giving encouragement and in particular my mother for providing a place of comfort and good meals. 


\begin{abstract}
In acoustic and electromagnetic scattering various methods for the reconstruction of the shape of an unknown object are examined. In particular, the multiwave range test is developed based upon the range test in the acoustic case. This method reconstructs the shape of the object from the data of many incident plane waves without the need of knowing the boundary condition of the unknown object. Strong connections between this method and other methods from shape reconstruction are presented. With this method an alternative approach to the singular sources method is developed. Further, the range test, based upon one incident plane wave, is carried over to the $3 \mathrm{D}$ electromagnetic case. For all methods under consideration numerical examples for the acoustic case $(2 \mathrm{D})$ and the electromagnetic case $(3 \mathrm{D})$ are presented.

We participated in the BMBF-funded project for the improvement of existing handheld mine detectors. Here, a full reconstructions of the metal parts of mines, under small modifications of the metal detector, is tried. This has the aim of significantly reduce the false alarm rate. During this research a program were developed and implemented, which simulates the setting in two-layered media using integral equations in a fast and efficient way. Also it provides two different methods of reconstructing the shape of the object. The Greens tensor for two-layered media were constructed via an new approach and adapted to the situation at hand.
\end{abstract}

\title{
Zusammenfassung
}

Im Rahmen von akustischer und elektromagnetischer Streuung werden verschiedene Verfahren zur Objektrekonstruktion untersucht. Insbesondere wird das neue Verfahren Multiwave Range Test aus dem Range Test im akustischen Fall entwickelt und dargestellt, welches aus mehreren einfallenden ebenen Wellen, ohne Kenntnis der Randbedingung auf dem unbekannten Rand, eine Rekonstruktion des gesuchten Objektes erstellen kann. Es werden tiefgreifende Zusammenhänge dieser Methode mit anderen Methoden der Objektrekonstruktion aufgezeigt. Dabei wird gezeigt und nachgewiesen, dass der Multiwave Range Test zu einer alternativen Form der Methode singulärer Quellen weiterentwickelt werden kann. Weiterhin wird der auf einer einfallenden Welle basierende Range Test in den elektromagnetischen Fall in drei Dimensionen übertragen. Für alle behandelten Methoden werden numerische Beispiele in der Akustik (2D) sowie in der Elektromagnetik (3D) gezeigt.

Wir nahmen am BMBF-geförderten Projekt für die Verbesserung von bestehenden, handgetragenen, Minensuchgeräten teil. Hierbei wird, unter leichter Modifikation des Minensuchgerätes, eine volle Rekonstruktion der zu suchenden Metallteile versucht. Dieses hat zum Ziel, die Falschalarmrate signifikant zu verbessern. Im Rahmen dieser Forschung wurde ein Programm entwickelt und implementiert, welches die gegebenen Situationen in zwei-geschichteten Medien schnell und effizient mittels Integralgleichungsmethoden simuliert, sowie mit zwei verschiedenen Variationen auch Objektrekonstruktionen liefert. Der benötigte Green'sche Tensor für ein zweigeschichtetes Medium wurde über einen neuartigen Zugang der Situation angepasst. 


\section{Contents}

1 Introduction 1

I Acoustic scattering 9

2 Setting and Tools 13

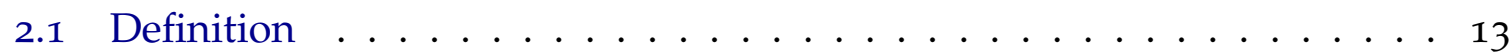

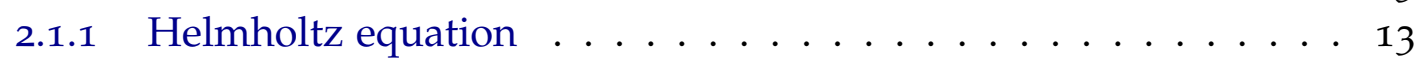

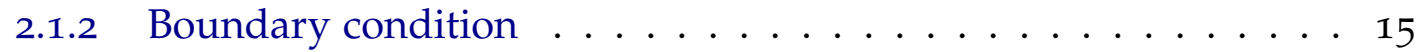

2.1.3 Radiation condition and far field pattern .......... 16

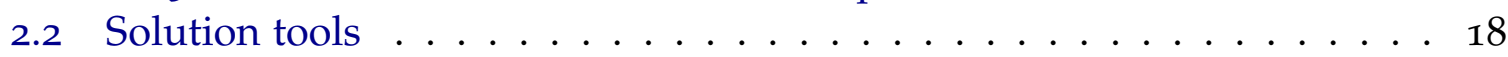

2.2.1 Fundamental solution . . . . . . . . . . . . . 18

2.2.2 Surface potentials . . . . . . . . . . . . . . . 19

3 Direct problem 23

3.1 Dirichlet or sound-soft problem . . . . . . . . . . . . . 25

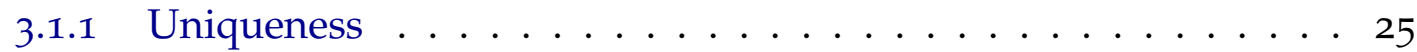

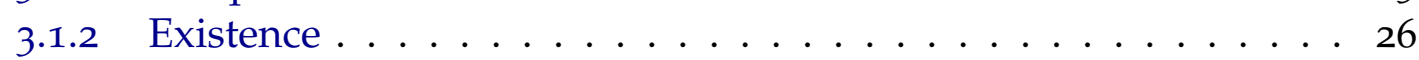

3.2 Neumann or sound-hard problem . . . . . . . . . . . . 27

3.2.1 Uniqueness . . . . . . . . . . . . . . . 27

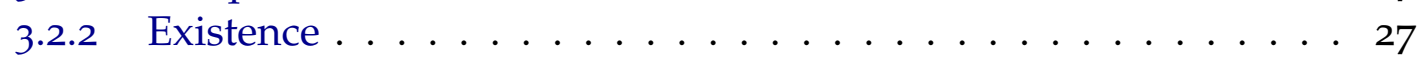

4 Inverse problem $\quad 29$

4.1 Range test . . . . . . . . . . . . . . . . . . . . . 29

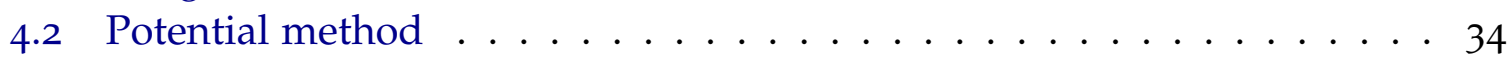

4.2.1 Modification using the range test . . . . . . . . 35

4.3 Multiwave range test . . . . . . . . . . . . . . . 37

4.4 Singular sources method - alternative . . . . . . . . . . . . 43

4.5 Relation of sampling methods under consideration . . . . . . . . . 44

5 Numerics $\quad 47$

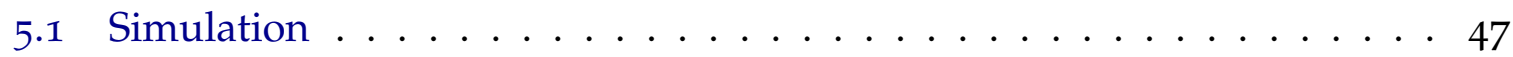

5.2 Choice of the test domains and regularisation parameters $\ldots \ldots . . .48$

5.3 Range test . . . . . . . . . . . . . . . . . . . . 49

5.4 Modified potential method . . . . . . . . . . . 50

5.5 Multiwave range test . . . . . . . . . . . . . $5^{1}$

5.6 Singular sources method - alternative . . . . . . . . . 52 
II Electromagnetic scattering $\quad 55$

6 Setting and tools $\quad 59$

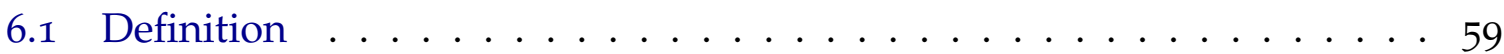

6.1.1 Time-dependent and time-harmonic Maxwell equations . . . . 59

6.1 .2 Transmission conditions . . . . . . . . . . . . . . 62

6.1 .3 Boundary condition .................. 62

6.1.4 Radiation condition and Rellich . . . . . . . . . . . . 63

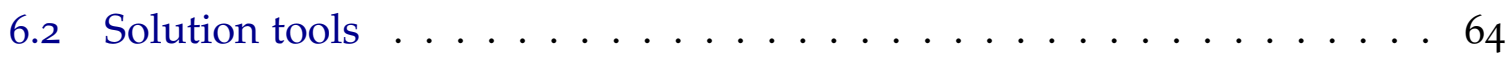

6.2.1 Fundamental solution and Green's tensor . . . . . . . . . . . . 65

6.2.2 Derivation of the Green's tensor . . . . . . . . . . . . . 66

6.2.3 Surface potentials . . . . . . . . . . . . . 73

6.3 Common settings for mine detection . . . . . . . . . . . 75

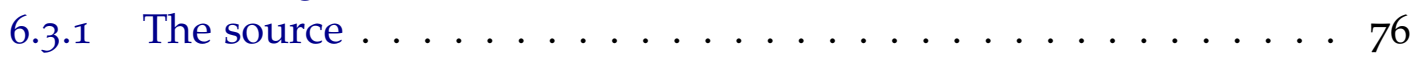

6.3 .2 The measurement . . . . . . . . . . . . . 77

6.3 .3 Physical constants . . . . . . . . . . . . . 78

7 Direct Problem $\quad 79$

7.1 Perfect conductor in a homogenous background medium . . . . . . 80

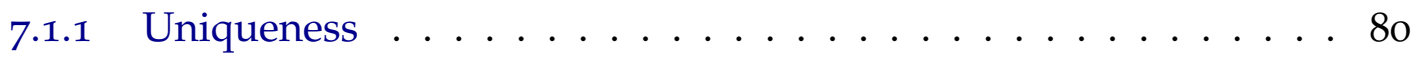

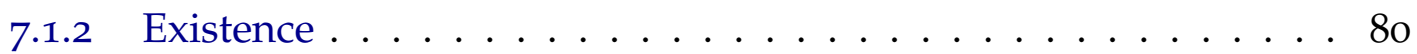

7.2 Perfect conductor in a layered background medium . . . . . . . . 83

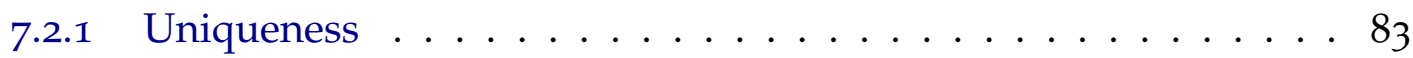

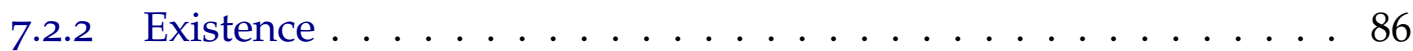

$7 \cdot 3$ Transmission problem in a homogeneous background medium . . . . . 87

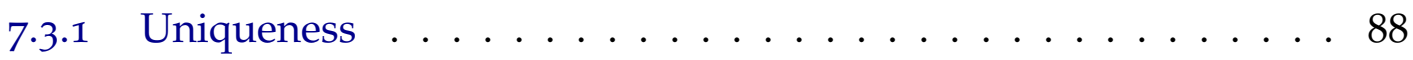

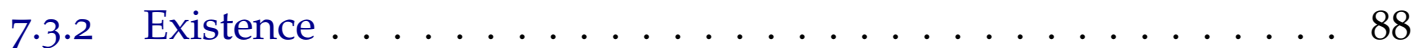

$7 \cdot 4$ Transmission problem in a layered background medium . . . . . . 91

$7 \cdot 4.1$ Uniqueness ......................... 91

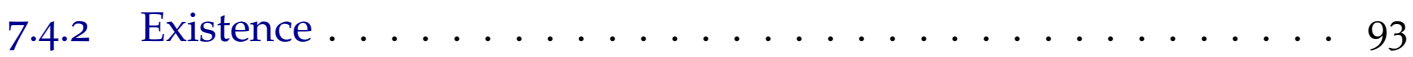

8 Inverse Problem $\quad 95$

8.1 An inverse optimisation problem . . . . . . . . . . . . . . 95

8.2 Nelder-Mead simplex method . . . . . . . . . . . . . . . . . . 96

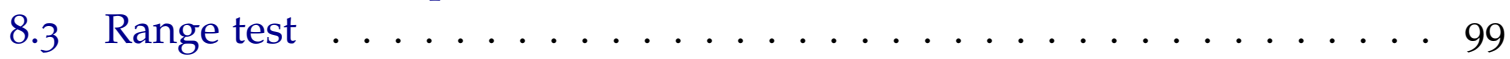

9 Numerics 105

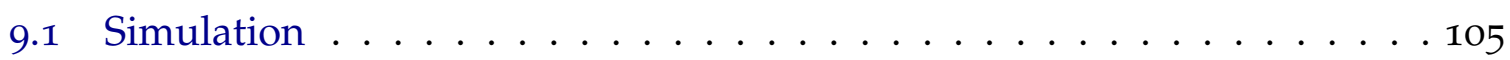

9.1.1 Rate of convergence . . . . . . . . . . . . . 108

9.2 Nelder-Mead simplex method . . . . . . . . . . . . . . . . . . 110

9.3 Range test ............................ . . . . . . . .

$\begin{array}{ll}\text { III Appendix } & 119\end{array}$ 
A Integral equations 121

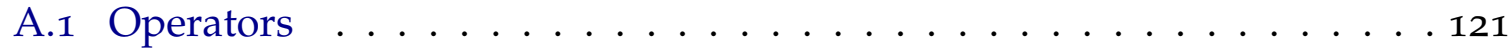

A.2 Riesz-Fredholm theory . . . . . . . . . . . . . . . . . . . . . . . . . . . . . . . . . . . . . .

A.3 Ill-posed problems . . . . . . . . . . . . . . . . 125

A.4 Tikhonov regularisation . . . . . . . . . . . 126

$\begin{array}{lr}\text { B Common tools for scattering theory } & 129\end{array}$

B.1 Green's theorem and formula . . . . . . . . . . . . . . . . . . . . . . . . . . . . . . . . . . . . . .

B.2 Stratton-Chu formulas . . . . . . . . . . . . . . . 132

B.3 Spherical Bessel and Hankel functions . . . . . . . . . . . 132

C Numerical treatment $\quad 139$

C.1 Nyström method . . . . . . . . . . . . . . . . . . . . 139

C.2 Fast Hankel transform . . . . . . . . . . . . . . . . . . 140

$\begin{array}{ll}\text { Bibliography } & 147\end{array}$

$\begin{array}{ll}\text { List of Figures } & 151\end{array}$

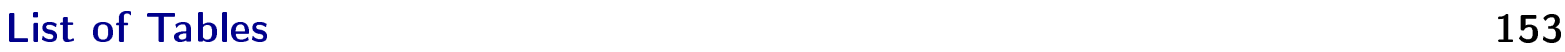

$\begin{array}{ll}\text { Nomenclature } & 155\end{array}$

$\begin{array}{ll}\text { Index } & 159\end{array}$

$\begin{array}{ll}\text { Curriculum Vitae } & 162\end{array}$ 
Contents

viii 


\section{Chapter 1}

\section{Introduction}

From a practical point of view, there is much interest in getting information about the interior of objects or subjects with a minimum of disturbance and interaction or even damaging the object or injuring the subject. The areas of interest are for example medical imaging, geophysical exploration and nondestructive testing. In particular, a goal would be to observe machines during their operation without the need of dismantling or stopping the machine. The interest often lies in finding the location and shape of inclusions or scatterers inside the domain of observation in a fast way, which yield good reconstructions possibly without prior information. These problems are covered by the scientific fields of acoustic or electromagnetic scattering. In this thesis, the focus lies on methods for solving the direct and the ill-posed inverse problem for problems from acoustic and electromagnetic scattering.

\section{Main topics}

In this work, the following two topics are presented.

- A new method called the multiwave range test is presented for the case of acoustic scattering. It is an extension of the range test originally developed for the acoustic case that is carried over to the case of electromagnetic scattering. It also leads to new views on existing methods and provides an alternative approach to the singular sources method.

- For the simulation of handheld mine detection a forward solver using integral equation methods is developed and implemented. The use of integral equation instead of the usual finite element methods (FEM) yields a reduction in complexity, as the problem can be solved from calculations on the boundary of the obstacle rather than the full three dimensional space.

\section{Range test and multiwave range test}

The range test that was introduced by Potthast, Sylvester and Kusiak (2003) [41] solves the inverse problem of locating and reconstructing the shape of an unknown object inside an observation area while using only the knowledge of one incident field and the measurement of the scattered field in some predefined domain or sphere 
around the observation area. Originally it handles only one incident time-harmonic wave in an acoustic setting which reconstructs a part of the scatterer, the so called convex scattering support. This method is carried over to the case of electromagnetic scattering. Furthermore, it is extended to a multiwave variant, i.e. it uses multiple incident waves, which allows reconstructions of the full shape of the obstacle, cf. [40].

Both methods belong to the class of sampling methods, which have in common that they are testing properties of the field or have other indicators to identify regions which are inside or outside the unknown object. The class of sampling methods (cf. [36]) contains, for example

- the linear sampling method from Colton and Kirsch (1996) [7],

- the factorisation method from Kirsch (1998) [18],

- the enclosure method from Ikehata (1999) [16],

- the singular sources method from Potthast (2000) [34],

- the no response test from Luke and Potthast (2002) [27].

Sampling methods are quick and powerful methods for the reconstruction of location and shape of obstacles which are usually faster than the simulation of one forward problem and which do not need the knowledge of the boundary condition. This missing information is usually compensated by measurements for a large number of different incident waves, but the range test and the no response test have been formulated for scattering of one incident wave, i.e. they need much less data than the other methods listed above, but their reconstructions are not as good as the methods using more data.

Range test. Consider the scattering of time-harmonic acoustic or electromagnetic waves by some, possibly multiply connected, scatterer $D$ in $\mathbb{R}^{m}$ for $m=2,3$. An acoustic incident wave $u^{i}$ gives rise to the scattered field $u^{s}$ with far field pattern $u^{\infty}$. In the electromagnetic case the incident electric field $E^{i}$ gives rise to the scattered field $E^{S}$ with nearfield $\left.E^{s}\right|_{M}$ on a measurement area $M$. Then, the basic idea of the range test is to determine the maximal set onto which the scattered field may be analytically extended. This is done via testing the solvability of the equations

$$
S_{\partial G}^{\infty} \varphi=u^{\infty} \quad \text { or } \quad P_{1, \partial G} a=\left.E^{S}\right|_{M},
$$

where $S_{\partial G}^{\infty}$ is the far field operator (2.23) and $P_{1, \partial G}$ the near field evaluation operator (7.4) with densities $\varphi, a$ on the boundary of some test domain $G \supset D$, in the respective cases. The complement of this set is a subset of the unknown scatterer $D$. Through testing with a fixed test shape for many different locations and taking the intersections for all test domains, for which the above equation is solvable, a reconstruction of the shape of the unknown obstacle is achieved. If the set of test domains is generated through translations of one fixed test domain, the testing can be done very efficiently. It should be pointed that this method does not deliver full 


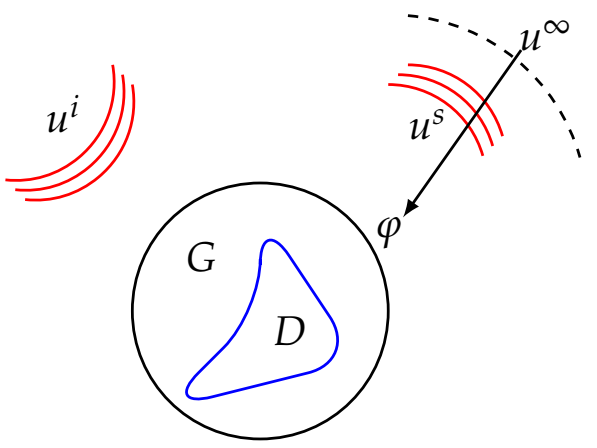

(a) Range test

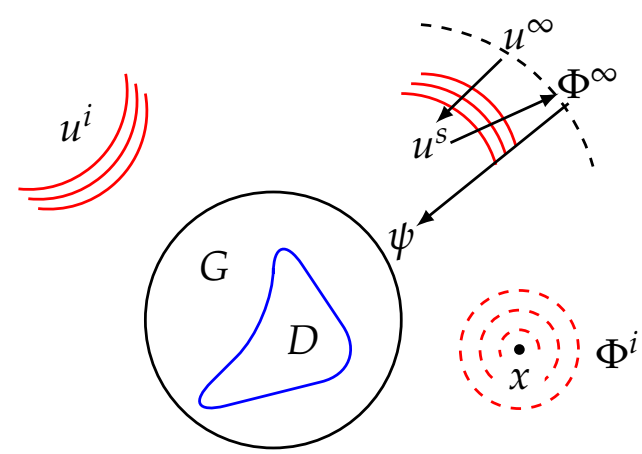

(b) Multiwave range test

Figure 1.1: Setup and idea of the range test (a) and the multiwave range test (b). Shown is one test domain $G$ containing the scatterer $D$. The arrows shows the steps to be taken for the method.

reconstructions of the shape of scatterers, because from the knowledge of only one wave and without knowing the boundary condition it is not possible to calculate the full shape of an unknown scatterer $D$. The idea of the method is shown for the acoustic case in a) of Figure 1.1. The electromagnetic case is analogous. Further, it can be used to modify the potential method [19-21] leading to convergence results and a better splitting of the parts of the method.

Multiwave range test. The range test is extended in the $2 \mathrm{D}$ case to a multiwave variant, for which full reconstructions of the shape of $D$ are possible. It is proven that if the available data consists of the far field patterns $u^{\infty}$ for all directions $d \in$ $\mathrm{S}:=\left\{x \in \mathbb{R}^{2}:\|x\|=1\right\}$ of incident plane waves, the data uniquely determines the unknown scatterer even if the boundary condition is not known (see [35]). The steps for the extension to a multiwave version of the range test are as follows. Similar to the range test one checks for the solvability of the equation above for each incident plane wave with direction $d \in \mathrm{S}$ and for all points $x$ in the region of observation $M$. If the equation is solvable the resulting density is used to calculate a scattered field $u^{S}(\cdot, d)$ via a single-layer operator $(2.21) S_{\partial G}$. Now, the mixed reciprocity relation (Theorem 3.0.9) states that

$$
u^{S}(x,-d)=c \Phi^{\infty}(d, x) \quad, d \in S, x \in M \backslash \bar{G},
$$

where $\Phi^{\infty}$ is the far field pattern of a point source and $c$ some constant. Then, the far field pattern of a point source is calculated from the measured far field pattern

$$
\Phi^{\infty}(d, x)=\frac{1}{c} S_{\partial G}\left(\left(S_{\partial G}^{\infty}\right)^{-1} u^{\infty}(\cdot,-d)\right)(x) \quad, d \in \mathbb{S}, x \in M \backslash \bar{G} .
$$

Now, given the far field pattern $\Phi^{\infty}(\cdot, x)$ for fixed $x$ on the set $\mathrm{S}$, the extensibility of this field into the exterior of $\mathbb{R}^{m} \backslash \bar{G}$ is tested in the same way as in the range test for one wave above, i.e. the range test is repeated, but now applied to $\Phi^{\infty}(\cdot, x)$ as far field pattern. This method can, in principle, reconstruct the full shape of the 


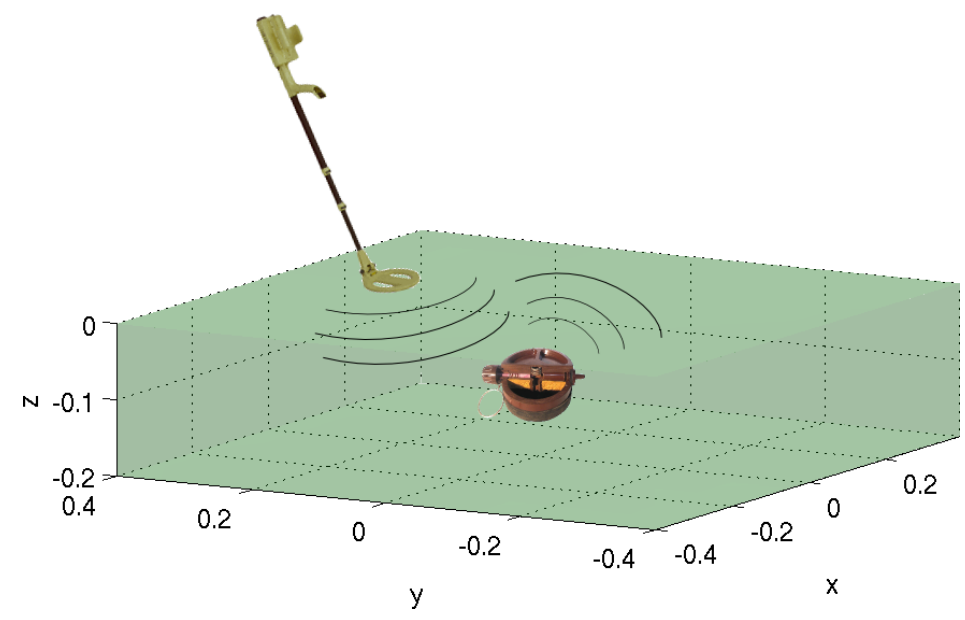

Figure 1.2: Common setting for mine detection with a handheld detector.

unknown obstacle. This is due to the fact that the scattered field $\Phi^{s}(x, x)$ of a point source located in $x$ becomes singular, and therefore also the corresponding density blows up, when $x$ approaches the boundary. The idea of the multiwave range test is illustrated in $b$ ) of Figure 1.1. The method then can be extended to an alternative approach of the singular sources method [34, 35] via using the density to evaluate the scattered field outside of the obstacle.

\section{Mine detection with handheld detectors}

The detection of metallic objects from remote measurements is an important application nowadays in, for example, nondestructive testing and detection of buried metallic objects. In this thesis the focus is on detection of mines with handheld mine detectors.

The research was done as part of the project "Metal detectors for Humanitarian Demining: Development potentials for data analysis and measurement techniques" which was funded by the german federal ministry of education and research (BMBF). It is a goal of the project to analyse possible improvements for handheld mine-detectors in searching for anti-person mines. These mines cover large areas mainly due to the increase of smaller asynchronous and civil conflicts. By the means of cheap handheld mine-detectors and better algorithms it is the hope to increase efficency of the mine-detection process and the removal of the dangerous mines. Four different models are used to simulate the mine-detection process. The best model of the four is a three-dimensional inverse electromagnetic scattering problem in a two-layered background medium with conducting obstacles. This setting is shown in Figure 1.2. An aim was to develop a fast forward solver which was done with an integral equations approach. The inverse problem is to find the location, size and rough shape of the unknown mine. The methods used was a simple gradient-free minimisation algorithm known as Nelder-Mead simplex method and the range test, as described above. Both methods are first steps taken towards more sophisticated methods which have the potential to greatly improve the correct detection of mines. 
For the simulation of a handheld mine detector the following setting is used. The mine detector is simulated to be composed of an emitter and receiver loop which are linked together in a static chassis. The emitter has roughly the form of a circle and the receiver loop has the shape of a "double-D". The handheld detector operates in the air and tries to find metallic objects, i.e. mines, beneath the ground. The environment can roughly be separated into two layers, the air and the earth. The conductivity $\sigma$ in the air is assumed to be negligible and in the earth $\sigma$ is assumed to have the approximately value of $0.05 \mathrm{~A} / \mathrm{Vm}$.

The mine detector scans through an roughly plane area $\mathcal{M}$ in a specific height above the ground. For every position $x \in \mathcal{M}$ the emitter sends out the incident electromagnetic field $E^{i}$, which is scattered by the buried objects. The scattered field $E^{S}$ from the objects induces a voltage $U$ inside the receiver loop.

The direct problem is solved with an integral equation approach which, due to the two-layered medium, needs a dyadic Green's tensor to correctly solve the problem. There are several forms of the dyadic Green's tensor in the literature, but here a new version specifically adapted to the problem at hand is derivated and implemented. It has the property that it allows complex and non-complex wave numbers and it uses two layers. This allows a compact theory and gives a good overview of the scattering problem itself. The results for the two-layered problem were published in [10].

\section{Outline and abstract of the thesis}

The thesis is split into three parts. The first part focuses on acoustic scattering in two dimensions and the multiwave range test, the second part on electromagnetic scattering and the simulation of the mine-detection problem, whereas the third part is the appendix which summarises some basic mathematical and numerical tools that are used throughout this work.

\section{Acoustic scattering}

The part of acoustic scattering is organised into four chapters. Chapter 2 introduces a partial differential equation, namely the Helmholtz equation, which is used to model the acoustic scattering problem. Furthermore it develops the basic properties and tools for the direct and inverse problems under consideration. The boundaryand radiation conditions are introduced. For the acoustic part the Dirichlet and Neumann boundary conditions are used, which correspond to either sound-soft and sound-hard boundary conditions. Section 2.2 covers the fundamental solution to the Helmholtz equation and the surface potentials and operators used for the solution of the boundary value problems.

Chapter 3 describes the direct acoustic scattering problem and its solution via an integral equation approach. The direct problem is to calculate the scattered field $u^{s}$ and the far field pattern $u^{\infty}$ from the knowledge of the scatterer and the incident field $u^{i}$. In the two sections of the chapter uniqueness and existence are proven for 
the scattering problems with either Dirichlet- or Neumann boundary conditions on the obstacle. These proofs follow the literature.

Chapter 4 presents the inverse problem under consideration and four methods to solve it. The inverse problem is to reconstruct the location, shape and properties of the unknown scatterer from the knowledge of the incident field $u^{i}$ and the far field pattern $u^{\infty}$. The first method introduced is the range test, which already is described above. Furthermore, extensibility properties and convergence are proven.

The second method in Section 4.2 is a modified version of the potential method of Kirsch and Kress (1986) (see [8], [19], [20] and [21]) with improved convergence properties. The basic idea of the original potential method is first to reconstruct the scattered field $u^{s}$ from its far field pattern $u^{\infty}$ by fitting the far field pattern of some single-layer potential on a test domain lying inside the unknown obstacle. Then, using a known incident field $u^{i}$ it is possible to search for the unknown scatterer as the zero set of the total field $u^{i}+u^{s}$. To obtain convergence in [8] Colton and Kress needed to combine the two steps into a nonlinear optimization procedure. With the range test it is possible to modify the potential method to obtain a convergence statement where the method is split into two separate steps - an ill-posed linear step and a well-posed nonlinear step.

The third method in Section 4.3 the range test and the potential method are used to construct the multiwave range test. The description of the method is stated above. Furthermore, extensibility properties and convergence are proven.

The fourth method in Section 4.4 is an alternative approach to the singular sources method which then uses the multiwave range test in the process. In its original versions [34], [35] the singular sources method is based on the point source method ([37] and [38]). Using the density calculated from the multiwave range test an approximation for the scattered field $\Phi^{s}(z, z)$ of an incident point source $\Phi(\cdot, z)$ in its source point $z$ can be calculated. Then the blow-up property

$$
\left|\Phi^{S}(z, z)\right| \rightarrow \infty, z \rightarrow \partial D
$$

can be used to find the unknown shape $\partial D$.

In Section 4.5 the relations between the methods are worked out in more detail. The range test is the most simple approach, whereas the more complex potential method may be based on the range test. Then, the multiwave range test can be understood to be based on the range test and the potential method. Finally, the multiwave range test can be extended to the singular sources method.

In Chapter 5 the numerical implementation is discussed and examples for all methods under consideration are presented. First, in Section 5.1 the forward solver is discussed in its discretized version and an example for the calculated scattered field is shown. Afterwards, as preparation for the reconstruction methods, the details of choosing test domains and regularisation parameters is described. The four following sections formulate the numerical implementation of the four methods discussed in Chapter 4 and show numerical examples for every method. 


\section{Electromagnetic scattering}

Analogously to the acoustic part, the part of electromagnetic scattering is organized into five chapters. Chapter 6 introduces the setting and the tools. First, in Section 6.1 the time-harmonic Maxwell equations are described and the necessary physical motivated boundary-, transmission- and radiation conditions are summed up. Second, Section 6.2 defines the fundamental solution and introduces the Green's tensor together with some important properties. In Section 6.2.2 the Green's tensor for the two-layered medium is derivated. Again, analogous to the acoustic case, the surface potentials and their properties are introduced in Section 6.2.3. For the application of handheld mine detection the settings for the sources, the measurements and the physical constants are described in Section 6.3.

In Chapter 7 the direct problem will be set up. The Four different problems are considered, whereas their complexity is getting greater and the last, is the best model under consideration to describe the situation of an handheld mine detection. These four direct problems are different in their domain and background settings and are summed up in the following list.

(HP) Homogeneous background medium with perfectly conducting obstacle.

(LP) Layered background medium with perfectly conducting obstacle.

(HT) Homogeneous background medium with a homogenous conducting obstacle.

(LT) Layered background medium with a homogeneous conducting obstacle.

To solve these four problems integral equation methods are used and the uniqueness and existence is proven in the sections of the Chapter 7 .

In Chapter 8 two inverse problems are stated and solved with the Nelder-Mead simplex method(see for example [25]) and the range test respectiveley. Given a planar measurement area which is "scanned" with an handheld mine detector gives induced voltages for every point in the area (as stated above in "Mine detection with handheld detectors"). From this data, both inverse problems state to find the location and shape of the unknown obstacle lying in the earth (lower half space).

For using the Nelder-Mead simplex method the problem is first reformulated as an optimisation problem in Section 8.1 and then the Nelder-Mead algorithm is stated and explained in Section 8.2. In Section 8.3 the range test is reformulated in the electromagnetic setting using nearfield data in the measurement area, the extensiblity of the scattered field and convergence is proven. The theory is motivated from the theory for the range test in the acoustic case.

Finally, Chapter 9 again covers the numerical implementation and results of the direct and inverse problems under consideration. Section 9.1 describes the discretization of the operators and of the Green's tensor and giving rates of convergence for the solver. A numerical example is chosen for the setting of the mine detection as if induced voltages are measured with a handheld mine detector in a known measurement grid. Then, using such simulated data, reconstructions with the Nelder-Mead simplex method of location and rough shape of spheres and ellipses for all four 
cases of direct problems, i.e. for all different background media and different forms of obstacles, are shown in Section 9.2. The numerical examples for the range test in Section 9.3 are shown as numerical proof of concept for obstacles in the setting of homogeneous background media and with nearfield data of the electric field rather than the induced voltages.

The Appendix gives a short introduction and brief summary of some used mathematical tools such as Riesz-Fredholm theory and Tikhonov regularisation. In Section C.2 a fast Hankel transform is introduced which is used intensively in the calculations for the Green's tensor.

\section{Spaces and Notation}

Some important spaces for this work are briefly summed up in this section. The spaces of l-times continuously differentiable functions on $D \in \mathbb{R}^{m}$ are denoted by $C^{l}(D)$. The natural numbers with zero are denoted as $\mathbb{N}_{0}:=\mathbb{N} \cup\{0\}$.

Throughout this work vectors will be denoted as follows. Considering the vectors $a=\left(a_{1}, a_{2}, a_{3}\right)$ and $b=\left(b_{1}, b_{2}, b_{3}\right)$ in $\mathbb{R}^{3}$ or $\mathbb{C}^{3}$ the bilinear scalar product is given by

$$
a \cdot b:=a_{1} b_{1}+a_{2} b_{2}+a_{3} b_{3}
$$

the conjugate complex of $a$ is denoted as $\bar{a}$ and the euclidean norm is written as

$$
|a|:=\sqrt{a \cdot \bar{a}} .
$$

The supremum norm of functions defined on a set $D \in \mathbb{R}^{3}$ is notated as

$$
\|\cdot\|_{\infty}=\|\cdot\|_{\infty, D}
$$

Let the partial derivatives be defined as

$$
D^{\alpha} f(x):=\frac{\partial^{|\alpha|} f(x)}{\partial x_{1}^{\alpha_{1}} \cdots \partial x_{d}^{\alpha_{d}}}
$$

with a multi-index $\alpha$.

Denote the spaces of $l$-times continously differentiable functions on a domain $D \subset$ $\mathbb{R}^{m}$ as

$$
C^{l}(D):=\left\{f \in C(D) \mid D^{\alpha} f \in C(D) \text { for }|\alpha| \leq l\right\} .
$$

For simplicity $C^{0}(D)$ is denoted by $C(D)$. The space of $l$-times Hölder continuous functions analogously is given as

$$
C^{l, \alpha}(D):=\left\{f \in C^{l}(D)\left|D^{\alpha} f \in C^{0, \alpha}(D) \forall\right| \alpha \mid=l\right\} .
$$




\section{Part I}

Acoustic scattering 

This part deals with the description of the scattering of incident time-harmonic acoustic waves on obstacles.

Chapter 2 focuses on the definition of the problem and some basics tools and properties. The second Chapter (Chapter 3) deals with the direct problem, the third is about the inverse problem (Chapter 4 ) and the fourth Chapter (Chapter 5) finally will describe the numerical implementations of the direct and inverse problems on chosen examples. 


\section{Chapter 2}

\section{Setting and Tools}

Here, the setting of the acoustic scattering under consideration is introduced. Further, for later use in the direct and inverse problems, the needed mathematical tools are developed. In Section 2.1 the Helmholtz equation is derivated, which solves the time-harmonic acoustic scattering problem. Also in Section 2.1 the boundary- and radiation conditions are introduced which complete the setting. Finally in Section 2.2 the fundamental solution and the surface potentials along some of their important properties are developed.

\subsection{Definition}

In the Definition of the problem, the focus lies in the derivation of the Helmholtz equation and its properties in the context of this work. To be able to properly define the scattering problem, also boundary conditions for the scatterer and radiation condition for the scattered field are defined.

\subsubsection{Helmholtz equation}

The Helmholtz equation can be used to describe the static state of acoustic scattering problems. The physicist Hermann Ludwig Ferdinand von Helmholtz (1821 - 1894) (see Figure 2.1) gave his name for this equation for his contributions in acoustics and electromagnetics.

For the physical motivation and derivation of the Helmholtz equation start with examining the propagation of sound waves of small amplitude in a homogeneous isotropic medium in $\mathbb{R}^{m}$. The medium can be viewed as an inviscid fluid. Then, define the velocity field $v:=v(x, t)$ the pressure $p:=p(x, t)$ the density $\rho:=\rho(x, t)$ and the specific entropy $S:=S(x, t)$ of the fluid. The motion of the fluid is described through Euler's Equation

$$
\frac{\partial v}{\partial t}+v \cdot \operatorname{grad}(v)+\frac{1}{\rho} \operatorname{grad}(p)=0,
$$

the equation of continuity

$$
\frac{\partial \rho}{\partial t}+\operatorname{div}(\rho v)=0
$$




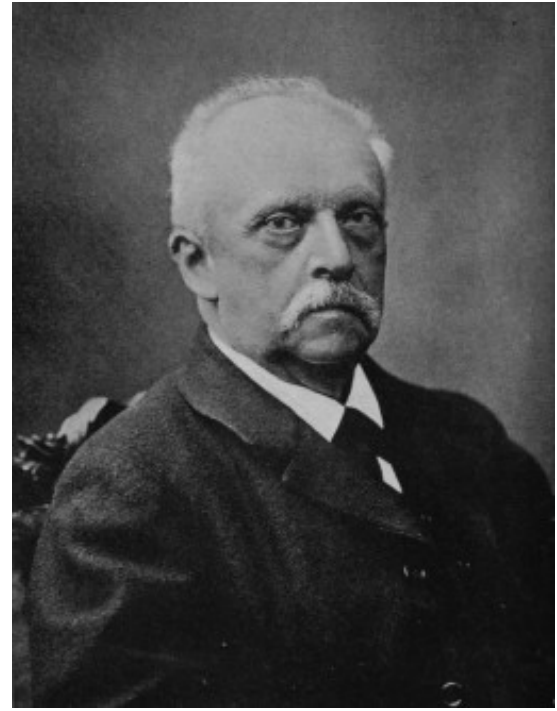

(a) Hermann Ludwig Ferdinand von Helmholtz

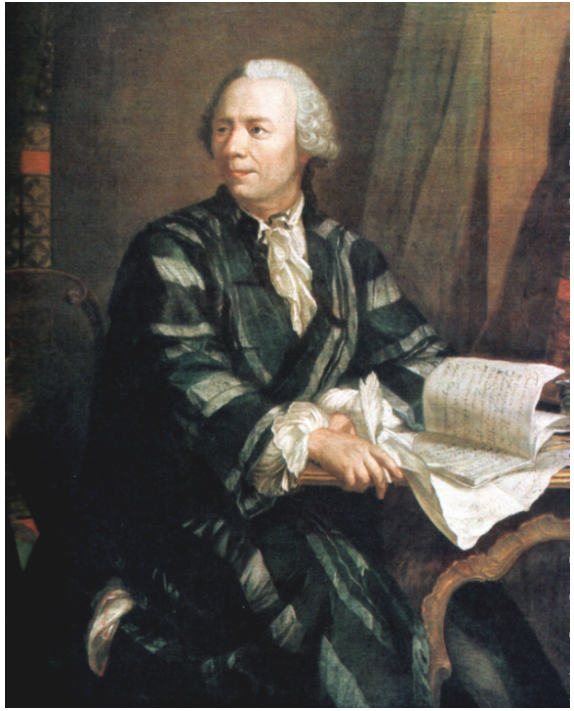

(b) Leonhard Euler

Figure 2.1: Hermann Ludwig Ferdinand von Helmholtz (1821 - 1894) and Leonhard Euler $(1707-1783)$

the state equation

$$
p=f(\rho, S),
$$

with some function $f$ depending on the nature of the fluid and finally with the adiabatic hypothesis

$$
\frac{\partial S}{\partial t}+v \cdot \operatorname{grad}(S)=0
$$

Euler's Equation originate from Leonhard Euler (1707 - 1783), a Swiss mathematician and physicist (see Figure 2.1). The equations correspond to the Navier-Stokes equations with zero viscosity. The function $f$ usually is given by the equation of state for ideal gas

$$
p=\rho(\gamma-1) e,
$$

with $\gamma$ the adiabatic index, and $e$ the internal energy.

Further look a the static state of the fluid. Then, there is $v_{0}=0$ and $p_{0}, \rho_{0}, S_{0}$ are constants. Assume that $v, p, \rho$ and $S$ are small perturbations of the static state, then linearise the Euler equation (2.1) to

$$
\frac{\partial v}{\partial t}+\frac{1}{\rho_{0}} \operatorname{grad}(p)=0
$$

the equation of continuity (2.2) to

$$
\frac{\partial \rho}{\partial t}+\rho_{0} \operatorname{div}(v)=0,
$$

and the state equation (2.3) to

$$
\frac{\partial p}{\partial t}=\frac{\partial f}{\partial \rho}\left(\rho_{0}, S_{0}\right) \frac{\partial \rho}{\partial t}
$$


Plug (2.5) into (2.6) and derivate with respect to the time $t$, multiply (2.4) with the Nabla-operator and merge the two resulting equations with eliminating the term with $\frac{\partial}{\partial t} \operatorname{div} v$. Then, with the speed of sound defined as

$$
c_{s}^{2}:=\frac{\partial f}{\partial \rho}\left(\rho_{0}, S_{0}\right),
$$

this leads to the wave equation given as

$$
\frac{1}{c_{S}^{2}} \frac{\partial^{2} p}{\partial t^{2}}=\Delta p
$$

From the linearised Euler equation (2.4) it can be concluded that there exist a velocity potential $\mathcal{U}(x, t)$ such that

$$
v=\frac{1}{\rho_{0}} \operatorname{grad} \mathcal{U}
$$

and

$$
p=-\frac{\partial \mathcal{U}}{\partial t}
$$

The velocity potential also satisfies the wave equation due to construction. For time harmonic acoustic waves of frequency $\omega>0$ the potential has the form

$$
\mathcal{U}(x, t)=\Re\left\{u(x) e^{-i \omega t}\right\}
$$

Plug this into the wave equation (2.8), derive and define the positive wave number

$$
\kappa:=\frac{\omega}{c_{S}} .
$$

Then the wave equation reduces to the reduced wave equation or Helmholtz equation

$$
\Delta u+\kappa^{2} u=0
$$

This is summarised in the following Definition.

Definition 2.1.1 (Helmholtz equation). The Helmholtz equation with positive wave number $\kappa:=\frac{\omega}{c}$ is given by

$$
\Delta u+\kappa^{2} u=0
$$

\subsubsection{Boundary condition}

Here, for impenetrable scatterers consider cases where the scatterer is either soundsoft or sound-hard which corresponds to either Dirichlet or Neumann boundary conditions on the obstacle $D$. The boundary conditions are conditions to the total field 


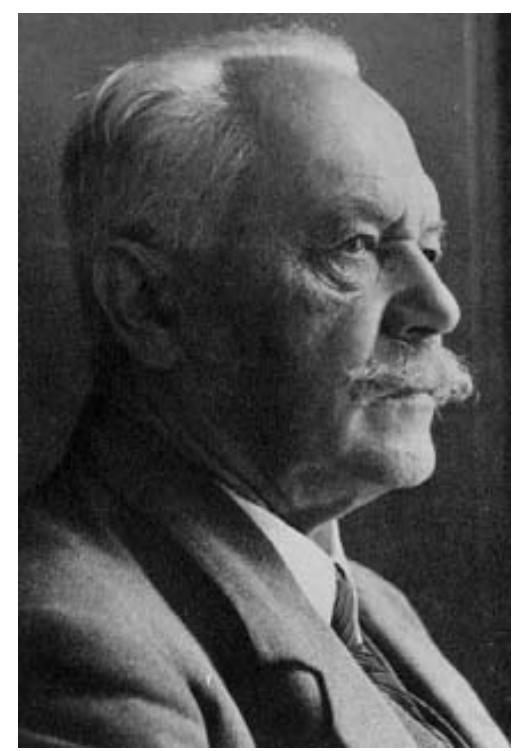

Figure 2.2: Arnold Johannes Wilhelm Sommerfeld (1868 - 1951).

on the boundary, where the total field is the sum from incident and scattered field. Then, the Dirichlet boundary condition is given by

$$
\left.u\right|_{\partial D}=0,
$$

and the Neumann boundary condition is given by

$$
\left.\frac{\partial u}{\partial v}\right|_{\partial D}=0
$$

where $v$ denotes the unit outward normal vector to $\partial D$.

\subsubsection{Radiation condition and far field pattern}

To be physically correct and have finite energy at infinity, a radiation condition for the solutions of the Helmholtz equations is necessary. For the Helmholtz equation Arnold Sommerfeld (1868-1951) (see Figure 2.2) formulated the Sommerfeld radiation condition [43]. Sommerfeld said in his own words:

The sources must be sources, and not energy sinks. Energy radiated from the sources must dissipate in the infinite; energy shall not flow from the infinite into the field singularities.

This corresponds to the term of outgoing waves.

Definition 2.1.2 (Sommerfeld radiation condition). A solution to the Helmholtz equation in $\mathbb{R}^{m}, m=2,3$ whose domain of definition contains the exterior of some sphere is called radiating if it satisfies the Sommerfeld radiation condition

$$
\lim _{|x| \rightarrow \infty}|x|^{\frac{m-1}{2}}\left(\frac{\partial}{\partial|x|}-i \kappa\right) u(x)=0
$$

uniformly in all directions $\hat{x}=\frac{x}{|x|}$. 
With the Sommerfeld radiation condition and Green's formula (Theorem B.1.2) it is now possible to formulate the far field pattern. The far field pattern resembles the physical situation that far away from the source and obstacle the field does behave like an outgoing spherical wave.

Theorem 2.1.3 (Far field pattern). Assume the bounded domain $D \in \mathbb{R}^{m}, m=2,3$ is the open complement of an unbounded domain of class $C^{2}$ and let $v$ denote the unit normal vector to the boundary $\partial D$ directed into the exterior of $D$. Radiating solutions $u^{s} \in C^{2}\left(\mathbb{R}^{m} \backslash\right.$ $\left.\bar{D} \cap C\left(\mathbb{R}^{m} \backslash D\right)\right)$ of the Helmholtz equation have the asymptotic behaviour of an outgoing spherical wave

$$
u^{\mathcal{S}}(x)=\frac{e^{i \kappa x}}{|x|^{\frac{m-1}{2}}}\left\{u^{\infty}(\hat{x})+\mathcal{O}\left(\frac{1}{|x|}\right)\right\},|x| \rightarrow \infty,
$$

uniformly for all directions $\hat{x}=x /|x|$. The function $u^{\infty}$ defined on the unit sphere $\mathrm{S}$ (or unit circle), is known as far field pattern, which is given by

$$
u_{\infty}(\hat{x})=\gamma_{m} \int_{\partial D}\left\{u^{s}(y) \frac{\partial e^{-i \kappa \hat{x} \cdot y}}{\partial v(y)}-\frac{\partial u^{s}}{\partial v}(y) e^{-i \kappa \hat{x} \cdot y}\right\} \mathrm{d} s(y), \quad \hat{x} \in \mathbf{S}
$$

with the constant

$$
\gamma_{m}:= \begin{cases}\frac{e^{i \pi / 4}}{\sqrt{8 \pi \kappa}} & , m=2 \\ \frac{1}{4 \pi} & , m=3\end{cases}
$$

Proof. See proof of Theorem 2.5 in [8].

A central lemma for this relationship and for the inverse problems at hand is Rellich's lemma which was named after the mathematician Franz Rellich (1906 - 1955). A photograph of him is shown in Figure 2.3.

Lemma 2.1.4 (Rellich). Assume that the bounded set $D$ is the open complement of an unbounded domain and let $u \in C^{2}\left(\mathbb{R}^{m} \backslash \bar{D}\right), m=2,3$ be a solution to the Helmholtz equation satisfying

$$
\lim _{r \rightarrow \infty} \int_{|x|=r}|u(x)|^{2} \mathrm{~d} s=0 .
$$

Then $u=0$ in $\mathbb{R}^{m} \backslash \bar{D}$.

Proof. The proof is identical to the proof of Theorem 2.11 of [8].

There is a one-to-one correspondance between far field patterns and radiating solutions of the Helmholtz equation, which is covered in the following theorem.

Theorem 2.1.5 (Radiating solution and far field pattern). Let the bounded set $D$ be an open complement of an unbounded domain and let $u \in C^{2}\left(\mathbb{R}^{m} \backslash \bar{D}\right)$ be a radiating solution to the Helmholtz equation for which the far field pattern vanishes identically $u^{\infty}=0$. Then $u=0$ in $\mathbb{R}^{m} \backslash \bar{D}$. 


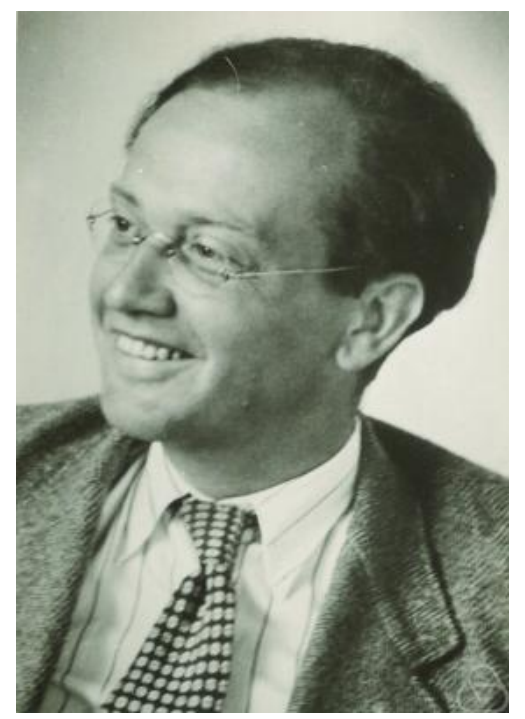

Figure 2.3: Franz Rellich (1906 - 1955).

Proof. From (2.15) deduce

$$
\lim _{r \rightarrow \infty} \int_{|x|=r}|u(x)|^{2} \mathrm{~d} s=\int_{\mathrm{S}}\left|u^{\infty}(\hat{x})\right|^{2} \mathrm{~d} s+\mathcal{O}\left(\frac{1}{r}\right) .
$$

The assumption $u^{\infty}=0$ on $\mathrm{S}$ implies that $u$ satisfies the assumptions of Rellich's lemma (Lemma 2.1.4). Hence, apply Rellich's Lemma to obtain $u=0$ in $\mathbb{R}^{m} \backslash \bar{D}$, which proves the theorem.

\subsection{Solution tools}

In this section the fundamental solution for the setting at hand are developed and analysed. Further, the surface potentials following Huygens' principle are introduced, which form the basis of the solution theory.

\subsubsection{Fundamental solution}

In studying the solutions of the Helmholtz equation it is possible to write down a radiating solution such that its superpositions can create any possible radiating solution to the Helmholtz equation. Such solutions in general are called fundamental solutions. With the aid of the fundamental solution of the Helmholtz equation many properties for the solutions of the Helmholtz equation can be deduced.

Definition 2.2.1 (Fundamental solution - Helmholtz equation). The free-space fundamental solution of the Helmholtz equation is given by

$$
\Phi(x, y):= \begin{cases}\frac{i}{4} H_{0}^{(1)}(\kappa|x-y|) & , \text { in } \mathbb{R}^{2} \\ \frac{1}{4 \pi} \frac{e^{i \kappa|x-y|}}{|x-y|} & , \text { in } \mathbb{R}^{3}\end{cases}
$$




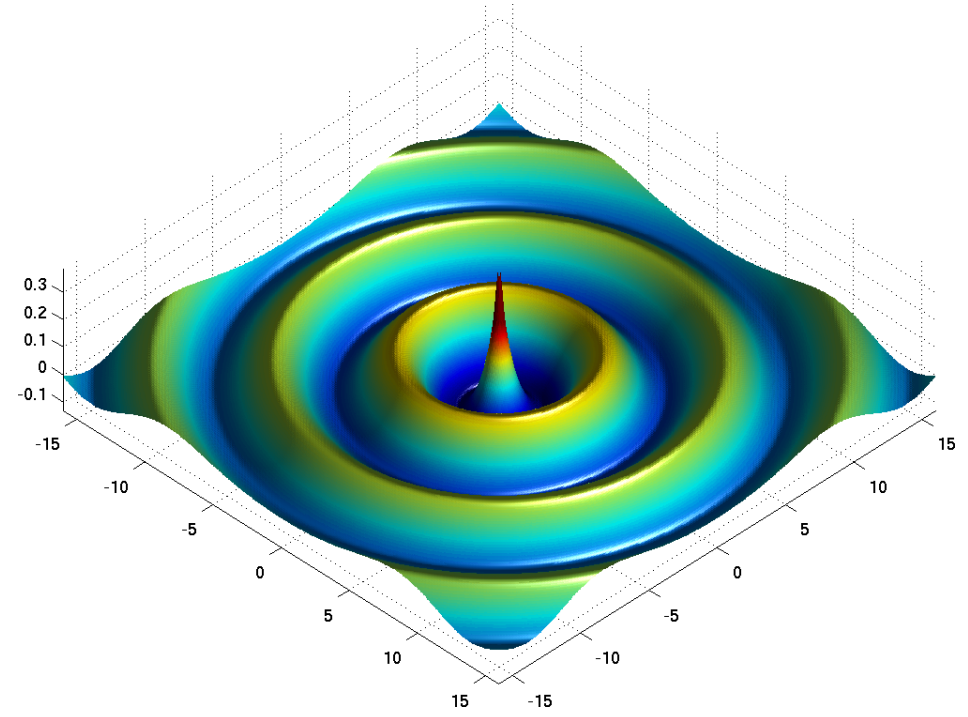

Figure 2.4: Real part of the fundamental solution for the Helmholtz equation in 2D.

with $H_{0}^{(1)}$ being the Hankel function of the first kind of order zero (see Section B.3).

Straightforward differentiation shows that the fundamental solution satisfies the Helmholtz equation in $\mathbb{R}^{m} \backslash\{y\}$. An example for the fundamental solution in $2 \mathrm{D}$ is shown in Figure 2.4.

\subsubsection{Surface potentials}

In this section the single-layer and double-layer surface potentials are introduced, which then are used to describe the direct problem later on. They use the fact that superpositions of the fundamental solution can be used to create any solutions of the underlying equation. Physically, they resemble Huygens principle, i.e. the idea to distribute point sources on the surface which approximate the real scattered field outside the obstacle. For given domains $D \subset \mathbb{R}^{m}$ with boundary $\partial D$ of class $C^{2}$ with normal vector $v$ which is oriented in the exterior and given functions $\varphi, \psi \in C(\partial D)$, the following operators can be defined. This notation is used throughout this work. The representation follows [23, chapter 6], [8, chapter 3] and [35].

Definition 2.2.2 (Single layer operator). The single layer potential is defined as

$$
u(x):=\int_{\partial D} \Phi(x, y) \varphi(y) \mathrm{d} s(y), x \in \mathbb{R}^{m} \backslash \partial D .
$$

The corresponding boundary operator $S_{\partial D}: C(\partial D) \mapsto C^{0, \alpha}(\partial D)$ is defined as

$$
\left(S_{\partial D} \varphi\right)(x):=2 \int_{\partial D} \Phi(x, y) \varphi(y) \mathrm{d} s(y), x \in \partial D,
$$

and is called the single layer operator. The integral exists as improper integral. 
The corresponding far field operator of the single-layer operator is given by

$$
\left(S_{\partial D}^{\infty} \varphi\right)(\hat{x}):=\gamma_{m} \int_{\partial D} e^{-i \kappa \hat{x} \cdot y} \varphi(y) \mathrm{d} s(y), \quad \hat{x} \in \mathrm{S}
$$

with the constant $\gamma_{m}$ defined in (2.17).

Remark. The potential is a solution to the Helmholtz equation (Definition 2.1.1) and satisfies the Sommerfeld radiation condition. Using Theorem 3.2 and 3.4 from [8] it can be seen, that the single-layer operator is compact.

The term single-layer comes from the idea of distributing monopoles on the boundary which can approximate the solution to the scattering problem.

Definition 2.2.3 (Double layer operator). The double layer potential is defined as

$$
v(x):=\int_{\partial D} \frac{\partial \Phi(x, y)}{\partial v(y)} \varphi(y) \mathrm{d} s(y), \quad x \in \mathbb{R}^{m} \backslash \partial D
$$

The corresponding boundary operator $K_{\partial D}: C^{0, \alpha}(\partial D) \mapsto C^{0, \alpha}(\partial D)$ is defined as

$$
\left(K_{\partial D} \varphi\right)(x):=2 \int_{\partial D} \frac{\partial \Phi(x, y)}{\partial \nu(y)} \varphi(y) \mathrm{d} s(y), x \in \partial D,
$$

and is called the double layer operator. The integral exists as improper integral.

In respect to the dual system $\langle C(\partial D), C(\partial D)\rangle$ defined by

$$
\langle\varphi, \psi\rangle:=\int_{\partial D} \varphi \psi \mathrm{d} s, \quad \varphi, \psi \in C(\partial D)
$$

using the $L^{2}$ scalar product, the operator $K_{\partial D}^{\prime}: C^{0, \alpha}(\partial D) \mapsto C^{0, \alpha}(\partial D)$ with

$$
\left(K_{\partial D}^{\prime} \psi\right)(x):=2 \int_{\partial D} \frac{\partial \Phi(x, y)}{\partial \nu(x)} \psi(y) \mathrm{d} s(y), \quad x \in \partial D,
$$

is the adjoint operator to the double layer operator. The integral exists as improper integral.

The normal derivative of the double layer operator $T_{\partial D}: C^{1, \alpha}(\partial D) \mapsto C^{0, \alpha}(\partial D)$ is given by

$$
\left(T_{\partial D} \varphi\right)(x):=2 \frac{\partial}{\partial \nu(x)} \int_{\partial D} \frac{\partial \Phi(x, y)}{\partial \nu(y)} \varphi(y) \mathrm{d} s(y), \quad x \in \partial D
$$

The integral exists as improper integral.

Remark. The potential is a solution to the Helmholtz equation (Definition 2.1.1) and satisfies the Sommerfeld radiation condition. Using Theorem 3.2 and 3.4 from [8] it can be seen that the operator $K$ is compact and that $K^{\prime}$ and $T$ are bounded operators.

The term double layer resembles the behaviour of the derivative of a monopole, which behaves like a dipole with polarisation orthogonal to the tangential plane. 
The potentials have jumps on the boundary $\partial D$ which are examined for continuous densities in the following theorems. These jump relations help setting up boundary integral equations which are equivalent to boundary value problems.

Theorem 2.2.4 (Jump relation single layer potential). The single-layer potential $u$ with density $\varphi \in C(\partial D)$ is continuous throughout $\mathbb{R}^{m}$ and on the boundary $\partial D$ it has the values

$$
u(x)=\int_{\partial D} \Phi(x, y) \varphi(y) \mathrm{d} s(y), \quad x \in \partial D
$$

and

$$
\frac{\partial u_{ \pm}}{\partial \nu}(x)=\int_{\partial D} \frac{\partial \Phi(x, y)}{\partial v(x)} \varphi(y) \mathrm{d} s(y) \mp \frac{1}{2} \varphi(x), \quad x \in \partial D
$$

where

$$
\frac{\partial u_{ \pm}}{\partial v}(x):=\lim _{h \rightarrow+0} v(x) \cdot \operatorname{grad} u(x \pm h v(x))
$$

is to be understood in the sense of uniform convergence on $\partial D$ and where the integrals exist as improper integrals. For some constant $C$ depending on $\partial D$ the following inequality holds

$$
\|u\|_{\infty, \mathbb{R}^{m}} \leq C\|\varphi\|_{\infty, \partial D}
$$

Proof. The proof is part of the proof of Theorem 3.1 of [8].

Theorem 2.2.5 (Jump relation double layer potential). The double-layer potential $v$ with density $\varphi \in C(\partial D)$ can be continously extended from $D$ to $\bar{D}$ and from $\mathbb{R}^{m} \backslash \bar{D}$ to $\mathbb{R}^{m} \backslash D$ with limiting values

$$
v_{ \pm}(x)=\int_{\partial D} \frac{\partial \Phi(x, y)}{\partial v(y)} \varphi(y) \mathrm{d} s(y) \pm \frac{1}{2} \varphi(x), \quad x \in \partial D
$$

where

$$
v_{ \pm}(x):=\lim _{h \rightarrow+0} v(x \pm h v(x))
$$

and where the integral exist as improper integral.

For some constant $C$ depending on $\partial D$ the following inequalities hold

$$
\begin{aligned}
\|v\|_{\infty, \bar{D}} & \leq C\|\varphi\|_{\infty, \partial D} \\
\|v\|_{\infty, \mathbb{R}^{m} \backslash D} & \leq C\|\varphi\|_{\infty, \partial D} .
\end{aligned}
$$

Furthermore, the limit

$$
\lim _{h \rightarrow+0}\left\{\frac{\partial v}{\partial v}(x+h v(x))-\frac{\partial v}{\partial v}(x-h v(x))\right\}=0, \quad x \in \partial D
$$

holds uniformly on $\partial D$.

Proof. The proof is part of the proof of Theorem 3.1 of [8]. 
Chapter 2 Setting and Tools 


\section{Chapter 3}

\section{Direct problem}

In this work only time-harmonic plane waves and point sources are used in the direct and inverse acoustic scattering problems under consideration. First, an acoustic plane wave is defined.

Definition 3.0.6 (Acoustic plane wave). Consider the time-harmonic acoustic case as in the derivation of the Helmholtz equation. A acoustic plane wave with direction of propagation $d$ in $\mathbb{R}^{m}$ where $m=2,3$ with frequency $\omega>0$ and wave number $\kappa$ (2.10) is given by

$$
\mathcal{U}(x, t)=e^{i(\kappa x \cdot d-\omega t)} .
$$

Considering the splitting of space and time as in (2.9), then the space dependent part is given by

$$
u(x)=e^{i \kappa x \cdot d} .
$$

Let $u^{i}(x)$ be the space-dependent part of an incident time-harmonic acoustic plane wave (3.o.6) in $\mathbb{R}^{m}$. Consider an impenetrable obstacle $D$ which is a bounded domain in $\mathbb{R}^{m}$ and has a boundary $\partial D$ which is of class $C^{2}$. The incident field $u^{i}$ is scattered at the obstacle $D$ and this gives a scattered field $u^{s}(x)$ that satisfies the Helmholtz equation (Definition 2.1.1). The scattered field should satisfy physical conditions, mainly its behaviour at infinity such that the total energy of the scattered wave is finite which leads to the Sommerfeld radiation condition (Definition 2.1.2). The total field is defined as the sum of the incident and the scattered field

$$
u=u^{i}+u^{s} .
$$

Now putting it all together and restrict the dimension to two or three a problem for acoustic scattering is set up in the following definition.

Definition 3.0.7 (Acoustic scattering problem). Consider an impenetrable obstacle $D$ which is a bounded domain in $\mathbb{R}^{m}$ with $m=2,3$ and has a boundary $\partial D$ which is of class $C^{2}$. Further, given a time-harmonic incident field $u^{i}$ which scatters on $D$ to a scattered field $u^{s}$

$$
u^{s} \in C^{2}\left(\mathbb{R}^{m} \backslash \bar{D}\right) \cap C\left(\mathbb{R}^{m} \backslash D\right),
$$



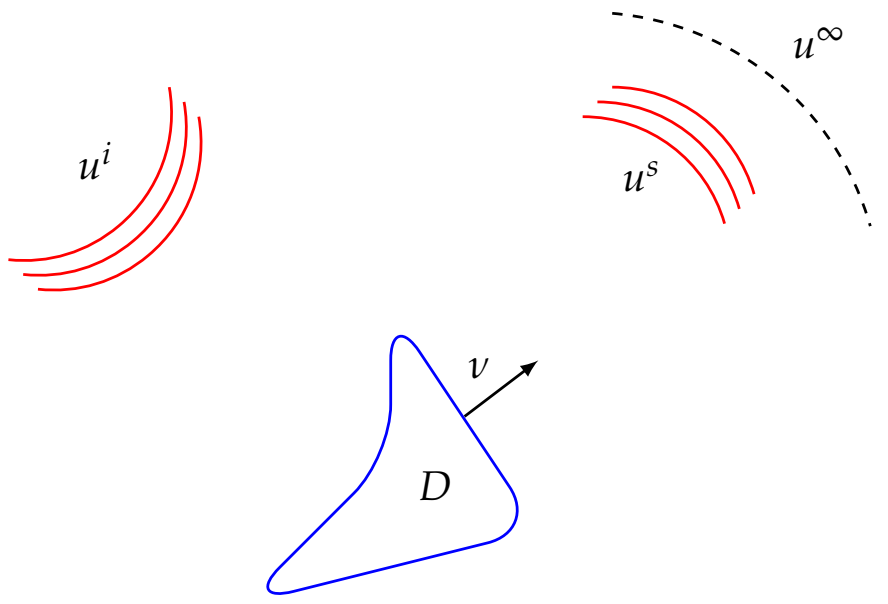

Figure 3.1: Setup of acoustic scattering. Scatterer $D$ with an normal $v$. Shown is the incident plane wave $u^{i}$, the scattered field $u^{s}$ and the far field pattern $u^{\infty}$.

that satisfies the Sommerfeld radiation condition (Definition 2.1.2) such that the total field

$$
u(x)=u^{i}(x)+u^{s}(x),
$$

solves the Helmholtz equation (Definition 2.1.1)

$$
\Delta u+\kappa^{2} u=0, \quad \mathbb{R}^{m} \backslash \bar{D},
$$

in $\mathbb{R}^{m} \backslash \bar{D}$ and fulfils a boundary condition. The far field pattern (Theorem 2.1.3) $u^{\infty}$ is given via the scattered field $u^{s}$.

The setup of the acoustic scattering is shown in Figure 3.1. The scatterer $D$ with normal $v$, the incident plane wave $u^{i}$, the scattered wave $u^{S}$ and its far field pattern $u^{\infty}$ are shown.

The scattered fields and the far field patterns for the direct acoustic scattering problem have some symmetry properties which are commonly called reciprocity relations. These symmetry properties play an important role in both direct and inverse acoustic problems.

Theorem 3.o.8 (Far field reciprocity relation). Consider the setting of acoustic scattering (Definition 3.0.7) with sound-soft or sound-hard boundary condition on the scatterer. Then, the far field patterns for a scattered wave satisfy

$$
u^{\infty}(\hat{x}, d)=u^{\infty}(-d,-\hat{x}), \quad \hat{x}, d \in S
$$

Proof. See Theorem 3.13 of [8] for the sound-soft case. The sound-hard case works analogously. 
Moreover, there exists a reciprocity relation which shows symmetry-relations between the scattered field and the far field of a point source. This was first worked out in [35, Chapter 2]. For this the constant

$$
\gamma_{m}:= \begin{cases}\frac{e^{i \pi / 4}}{\sqrt{8 \pi \kappa}} & , m=2 \\ \frac{1}{4 \pi} & , m=3\end{cases}
$$

is needed.

Theorem 3.0.9 (Mixed reciprocity relation). Consider the setting of acoustic scattering (Definition 3.0.7) with sound-soft or sound-hard boundary condition on the scatterer. Then the far field $\Phi^{\infty}(\cdot, z)$ of scattering from a point source $\Phi(\cdot, z), z \in \mathbb{R}^{m} \backslash \bar{D}$ and the scattered wave $u^{S}(\cdot, d), d \in \mathrm{S}$ for a plane wave incidence satisfy

$$
\Phi^{\infty}(\hat{x}, z)=\gamma_{m} u^{\mathcal{S}}(z,-\hat{x}), \quad z \in \mathbb{R}^{m} \backslash \bar{D}, \hat{x} \in \mathbb{S}
$$

Proof. See proof of theorem 2.1.4 in [35].

\subsection{Dirichlet or sound-soft problem}

Now consider the direct acoustic scattering problem with an obstacle with sound-soft (2.12) boundary conditions.

Definition 3.1.1 (Direct scattering problem, Dirichlet). Let the setting be as in the acoustic scattering (Definition 3.0.7) problem. Then, given incident field $u^{i}$ and location, shape of the obstacle $D$ with Dirichlet (2.12) boundary condition, find the scattered field $u^{s}$ and the far field pattern $u^{\infty}$.

\subsubsection{Uniqueness}

For the uniqueness proof the following theorem is needed. It is taken from [8].

Lemma 3.1.2 (Extended Rellich). Let $u \in C^{2}\left(\mathbb{R}^{m} \backslash \bar{D}\right) \cap C\left(\mathbb{R}^{m} \backslash D\right.$ be a radiating solution to the Helmholtz equation with $\kappa>0$ which has a normal derivative in the sense of a uniform convergence and for which

$$
\Im \int_{\partial D} u \frac{\partial \bar{u}}{\partial v} \geq 0
$$

Then $u=0$ in $\mathbb{R}^{m} \backslash \bar{D}$.

Proof. From the identity (B.7) and (3.2) it follows that the prerequisite of Rellich's Lemma (Lemma 2.1.4) is fulfilled. Hence, applying the Lemma completes the proof.

Theorem 3.1.3 (Uniqueness, acoustic Dirichlet). The direct scattering problem with Dirichlet boundary condition has at most one solution. 
Proof. Let $u^{d}=u^{s, 1}-u^{s, 2}$ be the difference of two solutions with the same incident field $u^{i}$. Then $u^{d}$ satisfies the Helmholtz equation with homogeneous boundary condition on $\partial D$. Thus, the boundary data is $C^{1, \alpha}$ and from the Theorem 3.27 in [9] $u$ is continously differentiable up to the boundary from which immediately Lemma 3.1.2 can be used to complete the proof.

\subsubsection{Existence}

For the solution of the Dirichlet problem the approach of Brakhage-Werner [4] is used. Hence, the scattered field is represented as a combined single (2.21)- and double layer potential (2.24).

$$
u^{S}(x)=\int_{\partial D}\left\{\frac{\partial \Phi(x, y)}{\partial v(y)}-i \eta \Phi(x, y)\right\} \varphi(y) \mathrm{d} s(y), \quad x \in \mathbb{R}^{m} \backslash \partial D,
$$

with density $\varphi \in C^{1, \alpha}(\partial D)$ and the real coupling factor $\eta$ chosen as $\eta=\kappa$. The ansatz solves the scattering problem (Definition 3.1.1) if, due to the jump relations (Theorem 2.2.4) and the boundary condition (2.12), the density $\varphi$ satisfies the integral equation

$$
\varphi+K \varphi-i \eta S \varphi=-2 u^{i},
$$

where $S$ is the single-layer operator (2.22) and $K$ is the double-layer operator (2.25).

Theorem 3.1.4 (Existence, acoustic Dirichlet). The scattering problem in Definition 3.1.1 has a unique solution. Further, the operator which maps the boundary data onto the solution is continuous from $C^{1, \alpha}(\partial D)$ into $C^{1, \alpha}\left(\mathbb{R}^{3} \backslash D\right)$.

Proof. To establish existence of a solution to the integral equation (3.4), by the RieszFredholm theory (Theorem A.2.2) it suffices to show that $I+K-i \eta S$ is injective since $K$ and $S$ are compact operators (see Section 2.2.2). Let $\varphi$ be a solution to the homogeneous form of (3.4). Then $u^{s}$ satisfies the homogeneous boundary condition $u^{s}=0$ on $\partial D$. Therefore, by the uniqueness (Theorem 3.1.3) $u^{s}=0 \in \mathbb{R}^{m} \backslash \bar{D}$. The jump relations (Theorem 2.2.4) now yield

$$
-u_{-}=\varphi, \quad-\frac{\partial u_{-}}{\partial v}=i \eta \varphi \quad \text { on } \partial D
$$

Then from Green's first theorem (Theorem B.1.1) it follows that

$$
i \eta \int_{\partial D}|\varphi|^{2} \mathrm{~d} s=\int_{\partial D} u_{-} \frac{\partial u_{-}}{\partial v} \mathrm{~d} s=\int_{D}\left\{|\operatorname{grad} u|^{2}-\kappa^{2}|u|^{2}\right\} \mathrm{d} x
$$

Taking the imaginary part of the last equation implies that $\varphi=0$ on $\partial D$. Thus, injectivity of the operator $I+K-i \eta S: C^{1, \alpha}(\partial D) \mapsto C^{1, \alpha}(\partial D)$ is shown. Then by the Riesz-Fredholm-theory (Theorem A.2.2) the operator is bijective and has a bounded inverse, i.e. (3.4) possesses a solution and the solution depends continuously on the right hand side. Furthermore, using theorem 3.3 in [8] and (3.3) implies that $u$ belongs to $C^{1, \alpha}\left(\mathbb{R}^{m} \backslash D\right)$. 


\subsection{Neumann or sound-hard problem}

Now consider the direct acoustic scattering problem with an obstacle with soundhard (2.13) boundary conditions.

Definition 3.2.1 (Direct scattering problem, Neumann)). Let the setting be the acoustic scattering (Definition 3.0.7) problem. Then, given incident field $u^{i}$ and location, shape of the obstacle $D$ with Neumann (2.13) boundary condition, find the scattered field $u^{s}$ and the far field pattern $u^{\infty}$.

\subsubsection{Uniqueness}

Theorem 3.2.2 (Uniqueness, acoustic Neumann). The direct scattering problem with Neumann boundary condition has at most one solution.

Proof. The proof is analogous to the uniqueness proof (Theorem 3.1.3) for the acoustic scattering problem with Dirichlet boundary conditions.

\subsubsection{Existence}

For the solution of the Neumann problem the modified approach due to Panich [32] is used in the following way

$$
u^{s}(x)=\int_{\partial D}\left\{\Phi(x, y) \varphi(y)+i \frac{\partial \Phi(x, y)}{\partial v(y)}\left(S_{0}^{2} \varphi\right)(y)\right\} \mathrm{d} s(y), \quad x \in \mathbb{R}^{m} \backslash \partial D,
$$

with density $\varphi \in C^{0, \alpha}(\partial D)$ where $S_{0}$ denotes the single layer operator in the limit as $\kappa \rightarrow 0$. The ansatz solves the scattering problem (Definition 3.2.1) if, due to the jump relations (Theorem 2.2.4) and the boundary condition (2.13), the density $\varphi$ satisfies the integral equation

$$
\varphi-K^{\prime} \varphi-i T S_{0}^{2} \varphi=2 \frac{\partial u^{i}}{\partial v}
$$

where $K^{\prime}$ is the adjoint double layer operator (2.27) and $T$ is the normal derivative of the double layer operator (2.28).

Theorem 3.2.3 (Existence, acoustic Neumann). The scattering problem in Definition 3.2.1 has a unique solution. Further, the operator which maps the boundary data onto the solution is continuous from $C^{0, \alpha}(\partial D)$ into $C^{1, \alpha}\left(\mathbb{R}^{3} \backslash D\right)$.

Proof. To establish existence of a solution to the integral equation (3.6), by the RieszFredholm theory (Theorem A.2.2) it suffices to show that $I+K^{\prime}-i \eta T S_{0}^{2}$ is injective since $K^{\prime}-i \eta T S_{0}^{2}$ is a compact operator since $K^{\prime}, T$ are bounded and $S_{0}$ is compact (cf. Section 2.2.2). Let $\varphi$ be a solution to the homogeneous form of (3.6). Then $u^{s}$ satisfies the homogeneous boundary condition $\partial u^{s} / \partial v=0$ on $\partial D$. Therefore, by the uniqueness (Theorem 3.2.2) $u^{s}=0 \in \mathbb{R}^{m} \backslash \bar{D}$. The jump relations (Theorem 2.2.4) now yield

$$
-u_{-}=i \eta S_{0}^{2} \varphi, \quad-\frac{\partial u_{-}}{\partial v}=-\varphi \quad \text { on } \partial D
$$


Then, from Green's first theorem (Theorem B.1.1) it follows that

$$
i \eta \int_{\partial D}\left|S_{0} \varphi\right|^{2} \mathrm{~d} s=i \eta \int_{\partial D} \varphi S_{0}^{2} \bar{\varphi} \mathrm{d} s=\int_{\partial D} \overline{u_{-}} \frac{\partial u_{-}}{\partial \nu} \mathrm{d} s=\int_{D}\left\{|\operatorname{grad} u|^{2}-\kappa^{2}|u|^{2}\right\} \mathrm{d} x .
$$

Taking the imaginary part of the last equation implies that $S_{0} \varphi=0$ on $\partial D$. The single-layer potential $w$ corresponding to the operator $S_{0}$ with wave number $\kappa=0$ and density $\varphi$ is continuous throughout $\mathbb{R}^{m}$, harmonic in $\mathbb{R}^{m} \backslash \partial D$ and in $D$ and vanishes on $\partial D$ and at infinity. Therefore, by the maximum-minimum principle for harmonic functions, it follows that $w=0$ in $\mathbb{R}^{m}$ and the second scalar jump relation (Theorem 2.2.4) yields $\varphi=0$. Thus, injectivity of the operator $I-K^{\prime}-i \eta T S_{0}^{2}$ : $C^{0, \alpha}(\partial D) \mapsto C^{0, \alpha}(\partial D)$ is shown. Then by the Riesz-Fredholm-theory (Theorem A.2.2) the operator is bijective and has a bounded inverse, i.e. (3.6) possesses a solution and the solution depends continuously on the right hand side. Furthermore, using Theorem 3.3 in [8] and (3.5) implies that $u$ belongs to $C^{1, \alpha}\left(\mathbb{R}^{m} \backslash D\right)$. 


\section{Chapter 4}

\section{Inverse problem}

In the subsequent sections of this chapter some different methods are explained which then in their sum leads to the multiwave range test (Section 4.3). These are the range test (Section 4.1) and the potential method (Section 4.2). At last, the singular sources method (Section 4.4) is reached via the multiwave range test and is described in an alternative form of the original. For the following sections the single-layer operator (2.21) and its corresponding far field pattern (2.23) are used extensively.

\subsection{Range test}

This section describes the one-wave range test which is then intensively used in Section 4.2, Section 4.3 and Section 4.4.

For the range test the inverse problem at hand is given in the following definition.

Definition 4.1.1 (Acoustic inverse shape reconstruction). In the setting of the acoustic scattering (Definition 3.0.7) in the dimension $m=2$ the following is given:

- one incident plane wave $u^{i}$ from the direction $d \in \mathrm{S}$.

- an aperture of the measurement circle $\Lambda \subset \mathcal{S}$.

- the measured far field pattern $u^{\infty}$ on $\Lambda$.

Then find the shape of the unknown boundary $D$.

Remark. There is a simple way for the range test to improve the final reconstructed shape by using several incident waves. For every wave use the range test to get a reconstruction of the shape and then take the union of the reconstructions for a better reconstruction.

The basic idea of the range test [41] is to determine the maximal set on to which one scattered field may be analytically extended via the single-layer approach. Then, the complement of this set is a subset of the unknown scatterer $D$. However, the method does not deliver full reconstructions of the shape of scatterers. From the knowledge of one wave only and without the knowledge of the boundary condition there is no hope to calculate the full shape of an unknown scatterer $D$. If there is more data 


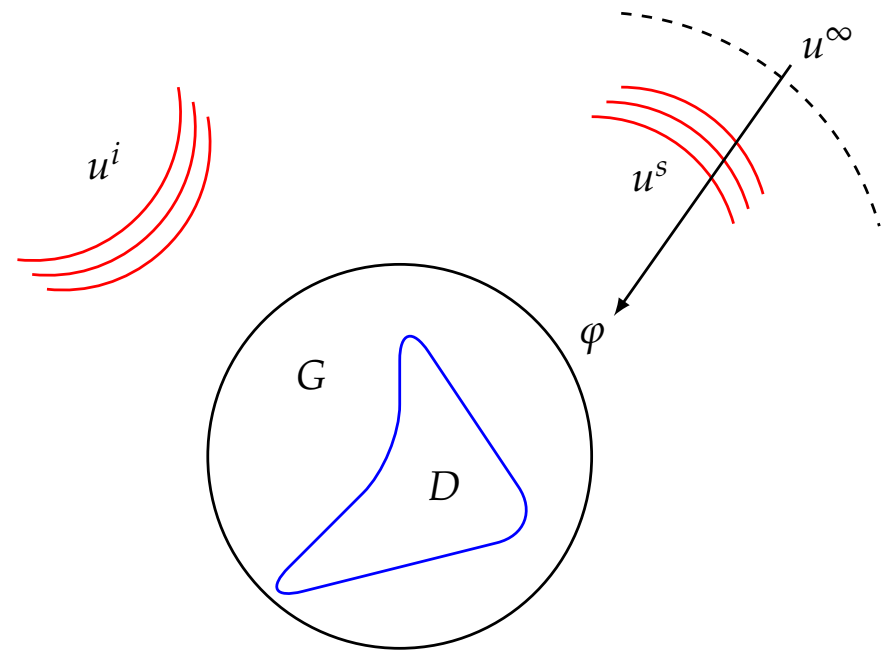

Figure 4.1: Setup and idea of the range test. Shown is one test domain $G$ containing the scatterer $D$. The idea is to get the density $\varphi$ from the measured $u^{\infty}$ from which to test the analytic extension of $u^{s}$ into $\mathbb{R}^{m} \backslash \bar{G}$.

available it is well known that the far field patterns $u^{\infty}(\hat{x}, d)$ for $\hat{x}, d \in \mathrm{S}$ uniquely determine the unknown scatterer even if the boundary condition is not known, see [35, Chapter 3].

Consider connected test domains $G$ of class $C^{2}$ with boundary $\partial G$ such that the interior Dirichlet problem for $G$ is uniquely solvable for the wave number $\kappa$ (i.e. $\kappa$ is not an interior Dirichlet eigenvalue). Then, by using the far field operator (2.23) of the single-layer potential (2.21) defined on the boundary $\partial G$ it can be evaluated if the scattered field $u^{s}$ is extensible into $\mathbb{R}^{m} \backslash \bar{G}$. The equation

$$
S_{\partial G}^{\infty} \varphi=u^{\infty}(\cdot, d)
$$

is solvable if $u^{s}$ can be analytically extended into $\mathbb{R}^{m} \backslash \bar{G}$ and it is not solvable if it cannot be analytically extended into $\mathbb{R}^{m} \backslash \bar{G}$. Thus, the solvability of the ill-posed integral equation (4.1) can be used as a criterion for the analytic extensibility of $u^{S}$ into $\mathbb{R}^{m} \backslash \bar{G}$. This idea and the setup for the range test is shown in Figure 4.1.

For the numerical implementation of the ill-posed equation (4.1) the unbounded inverse $\left(S_{\partial G}^{\infty}\right)^{-1}$ of $S_{\partial G}^{\infty}$ needs to be regularised. Using the Tikhonov regularisation (Theorem A.4.3) the regularised inverse is given by

$$
R_{\alpha}:=\left(\alpha I+S_{\partial G}^{\infty, *} S_{\partial G}^{\infty}\right)^{-1} S_{\partial G}^{\infty} .
$$

If the equation (4.1) is solvable, then the norm $\left\|\varphi_{\alpha}\right\|_{L^{2}}$ of

$$
\varphi_{\alpha}:=R_{\alpha} u^{\infty}(\cdot, d)
$$

will be bounded in the limit $\alpha \rightarrow 0$. If equation (4.1) does not admit a solution, then

$$
\left\|\varphi_{\alpha}\right\|_{L^{2}} \rightarrow \infty, \quad \alpha \rightarrow 0,
$$

i.e. for $\alpha \rightarrow 0$ the norm of the density will tend to infinity. 
The behaviour of the norm of the density is used to test the extensibility of the field $u^{S}$ by calculation of the norm $\left\|\varphi_{\alpha}\right\|$ for solutions with a number of different test domains $G$ and comparing $\left\|\varphi_{\alpha}\right\|$ with some cut-off constant $C$. If for sufficiently small (fixed) regularisation parameter $\alpha$ there is $\left\|\varphi_{\alpha}\right\| \leq C$, then it is concluded that the equation (4.1) is solvable. If in this case $\left\|\varphi_{\alpha}\right\|>C$, then conclude that it is unsolvable. In the case of solvability of (4.1) conclude that $u^{S}$ is analytically extensible into $\mathbb{R}^{m} \backslash \bar{G}$.

Now, to write down a proof for this behaviour first define the so called scattering support and show some of the properties. For a more complete investigation see [24].

Definition 4.1.2 (Scattering support). A domain $\Omega$ supports $u^{\infty}$ if the corresponding $u^{S}$ can be continued to solve the Helmholtz equation in $\mathbb{R}^{m} \backslash \bar{\Omega}$.

Let the incident field have the wave number $\kappa$. Then, the intersection of all convex $\Omega$ which support $u^{\infty}$ is called the convex scattering support or $\mathrm{cS}_{\mathcal{K}} \operatorname{supp} u_{\infty}$ of the far field $u^{\infty}$.

Lemma 4.1.3 (Supporting intersections). Let $\Omega_{1}$ and $\Omega_{2}$ be convex sets which support the same far field $u^{\infty}$. Then, $\Omega_{1} \cap \Omega_{2}$ supports $u^{\infty}$.

Proof. See proof of Lemma 3.1 of [41].

Lemma 4.1.4 (Properties of the convex scattering support). The convex scattering support has the following properties

1. if $u^{\infty} \neq 0$ then $\mathrm{cS}_{\kappa} \operatorname{supp} u_{\infty}$ is not empty.

2. Let $u^{i}$ be the incident field illuminating a scatterer with convex hull $\Omega$ which generates the far field pattern $u^{\infty}$. Then $\mathrm{cS}_{\kappa}$ supp $u_{\infty} \subset \bar{\Omega}$.

3. The convex scattering support $\mathrm{cS}_{\kappa}$ supp $u_{\infty}$ contains all the singularities of the scattered field $u^{s}$ which lie in the closure of the unbounded component of the complement of the convex hull of the support of the scatterer.

Proof. See proof of Lemma 3.3 of [41].

With this definition and the Lemmata the Theorem for the extensibility properties and its proof can be formulatied.

Theorem 4.1.5 (Extensibility properties). Assume that $\mathrm{cS}_{\kappa}$ supp $u_{\infty} \subset G$. Then the field $u^{s}$ can be analytically extended up to $\mathbb{R}^{m} \backslash G$, i.e. the $L^{2}$-norms of the densities $\varphi_{\alpha}$ solving (4.1) on $\partial G$ are bounded.

If $\mathrm{cS}_{\kappa}$ supp $u_{\infty} \not \subset G$, then the field $u^{s}$ cannot be extended up to $\mathbb{R}^{m} \backslash G$, i.e. the $L^{2}$-norms of the densities $\varphi$ solving (4.1) on $\partial G$ will not be bounded.

Proof. case $\mathrm{cS}_{\mathcal{K}}$ supp $u_{\infty} \subset G$ : The field $u^{s}$ with the far field pattern $u^{\infty}$ can be analytically extended into the open exterior of the domain $G$ and into the neighbourhood of $\partial G$. The solution to the equation

$$
S \varphi=u^{S} \quad \text { on } \partial G
$$


is unique since $S$ maps $L^{2}(\partial G)$ bijectively into $L^{2}(\partial G)$ ([8, Theorem 3.6]). On the boundary $\partial G$, the single layer potential $v$ now coincides with $u^{s}$ and by the solution of the exterior Dirichlet problem for the domain $G$ it coincides on $\mathbb{R}^{m} \backslash G$. Thus, the far field pattern $S^{\infty} \varphi$ of the single layer potential $v$ and $u^{\infty}$ coincide, i.e. $u^{\infty}$ is in the range of $S^{\infty}$. Furthermore, there exists an $\varphi \in L^{2}(\partial G)$ such that $S_{\partial G}^{\infty} \varphi=u^{\infty}$. Then, from the Tikhonov Theorem (Theorem A.4.3) the following holds

$$
\begin{aligned}
\lim _{\alpha \rightarrow 0} \varphi_{\alpha} & =\lim _{\alpha \rightarrow 0}\left(\alpha I+S_{\partial G}^{\infty, *} S_{\partial G}^{\infty}\right)^{-1} S_{\partial G}^{\infty, *} u^{\infty} \\
& =\lim _{\alpha \rightarrow 0}\left(\alpha I+S_{\partial G}^{\infty, *} S_{\partial G}^{\infty}\right)^{-1} S_{\partial G}^{\infty, *} S_{\partial G}^{\infty} \varphi \\
& =\varphi,
\end{aligned}
$$

thus $\left\|\varphi_{\alpha}\right\|$ for $\alpha \rightarrow 0$ is bounded.

case $c S_{\kappa}$ supp $u_{\infty} \not \subset G$ : Assume that there is a constant $C$ such that $\varphi_{\alpha}$ is bounded for sufficiently small $\alpha>0$. Then, there is a sequence $\alpha_{j} \rightarrow 0$ for $j \rightarrow \infty$ such that the weak convergence $\varphi_{\alpha_{j}} \rightarrow \tilde{\varphi}$ for $j \rightarrow \infty$ holds with some element $\tilde{\varphi} \in L^{2}(\partial G)$. The bounded linear integral operator $S_{\partial G}^{\infty}$ maps the weakly convergent sequence into a strongly convergent sequence, i.e.

$$
S_{\partial G}^{\infty} \varphi_{\alpha_{j}} \rightarrow S_{\partial G}^{\infty} \tilde{\varphi}
$$

for $j \rightarrow \infty$ and with $\tilde{u}^{\infty} \in S_{\partial G}^{\infty}\left(L^{2}(\partial G)\right)$ defined as

$$
\tilde{u}^{\infty}:=S_{\partial G}^{\infty} \tilde{\varphi}
$$

Then from the Tikhonov regularisation

$$
\left.\left(\alpha_{j} I+S_{\partial G}^{\infty, *} S_{\partial G}^{\infty}\right) \varphi_{\alpha_{j}}\right)=S_{\partial G}^{\infty, *} u^{\infty}
$$

passing to the limit $j \rightarrow \infty$ this leads to

$$
S_{\partial G}^{\infty, *} S_{\partial G}^{\infty} \tilde{\varphi}=S_{\partial G}^{\infty, *} u^{\infty}
$$

and from the definition of $\tilde{u}^{\infty}$ this yields

$$
S_{\partial G}^{\infty, *} \tilde{u}^{\infty}=S_{\partial G}^{\infty, *} u^{\infty},
$$

which implies $\tilde{u}^{\infty}=u^{\infty}$ and thus $u^{\infty} \in S_{\partial G}^{\infty}\left(L^{2}(\partial G)\right)$. Then there is a function $\varphi \in L^{2}(\partial G)$ such that $S_{\partial G}^{\infty} \varphi=u^{\infty}$. Using the single layer potential $v$ with density $\varphi$ the field $u^{s}$ can be analytic extended into $\mathbb{R}^{m} \backslash \bar{G}$. Thus $G$ supports $u^{\infty}$ which leads to the contradiction $\mathrm{cS}_{\mathcal{K}}$ supp $u_{\infty} \subset \bar{G}$ and therefore $\varphi_{\alpha}$ can not be bounded for $\alpha \rightarrow 0$.

From this Theorem and the description of the beginning of this section an algorithm for the range test can be formulated. 
Algorithm 1 (Range test). Let $\mathcal{J}$ be a finite Index set and further let $\mathcal{N}:=\left\{G^{(j)}: j \in \mathcal{J}\right\}$ be the set of test domains $G^{(j)}$ which are of class $C^{2}$ such that the interior homogeneous Dirichlet problem does have only the trivial solution.

Then, use (4.2) to calculate the indicator function

$$
\mu_{j}:=\left\|R_{\alpha} u^{\infty}\right\|_{L^{2}(\partial G)},
$$

for all $G \in \mathcal{N}$. This is used to test whether the scattered field $u^{s}$ can be analytically extended into $\mathbb{R}^{m} \backslash \overline{G^{(j)}}$, and if so call the domain $G^{(j)}$ a positive test domain. Finally, take the intersection of all positive test domains to calculate a subset of the unknown scatterer $D$ by

$$
D_{r e c}:=\bigcap_{\mu_{j}<C} \bar{G}_{j}
$$

with a chosen cutoff constant $C$.

A Theorem of convergence is stated here.

Theorem 4.1.6 (Convergence of the range test). Let $M$ be a domain with the property $\mathrm{cS}_{\kappa}$ supp $u_{\infty} \subset \bar{M}$. Using appropriate increasing sets of sampling domains $\mathcal{N}$, there exists a decreasing sequence of domains $M_{k}, k \in \mathbb{N}$ with $\mathrm{cS}_{\kappa}$ supp $u_{\infty} \subset \bar{M}_{k}$ such that for each domain $M$ and for all sufficiently large $k \in \mathbb{N}$ it holds that $M_{k} \subset M$.

Proof. See second part of proof of Theorem 4.2 in [41].

The range test does not use the boundary condition and the method can be applied to all kinds of objects with different or mixed boundary conditions. However, from the knowledge of the far field pattern for one incident wave it can not reconstruct the full shape of $D$. For example, testing the extensibility of the field $u^{s}$ into the exterior of convex test domain $G$ it merely constructs the convex scattering support of $D$, which is a subset of $D$. In special situations, for example for convex polygonal scatterers, this subset coincides with the scatterer itself. However, in general the reconstructed set will be only a part of the scatterer. Later in section 4.3 the extension to the range test will reconstruct the full shape of the scatterer in the setting with multiple waves.

Efficiency. The range test needs to solve linear integral equations for a large number of test domains. Setting up the integral operator for each test domain can make the algorithm very time consuming.

However, with the following method it is possible to efficiently calculate (4.2) for many test domains $G^{(j)}$, where $S_{\partial G^{(j)}}^{\infty}$ only needs to be set up for one initial test domain $G_{0}$.

Theorem 4.1.7 (Shifted operators). Let the domain $G_{0}$ be chosen such that the interior Dirichlet problem is uniquely solvable for the wave number $\kappa$. Let the test domains $G(x)$ be constructed from $G_{0}$ by translation

$$
G(x):=G_{0}+x
$$


with vector $x \in \mathbb{R}^{m}$. Then, with $\tilde{\varphi}(y):=\varphi(y+x)$ the following holds

$$
\left(S_{\partial G(x)}^{\infty} \varphi\right)(\hat{x})=\gamma_{m} e^{-i \kappa \hat{x} \cdot x}\left(S_{\partial G_{0}}^{\infty} \tilde{\varphi}\right)(\hat{x}) .
$$

Thus, the translation of the domain $G_{0}$ provides a quick way to calculate the solution of the corresponding integral equations.

Proof. Calculating

$$
\begin{aligned}
\left(S_{\partial G(x)}^{\infty} \varphi\right)(\hat{x}) & =\gamma_{m} \int_{\partial G(x)} e^{-i \kappa \hat{x} \cdot y} \varphi(y) \mathrm{d} s(y) \\
& =\gamma_{m} e^{-i \kappa \hat{x} \cdot x} \int_{\partial G_{0}} e^{-i \kappa \hat{x} \cdot y} \tilde{\varphi}(y) \mathrm{d} s(y) \\
& =\gamma_{m} e^{-i \kappa \hat{x} \cdot x}\left(S_{\partial G_{0}}^{\infty} \tilde{\varphi}\right)(\hat{x}),
\end{aligned}
$$

finishes the proof.

Remark. It is sufficient to set up one version of the operator $S_{\partial G_{0}}^{\infty}$ and obtain the regularised solutions $\varphi_{\alpha}$ for all test domains $G(x)$ by multiplication with the exponential factor $e^{-i \kappa \hat{x} \cdot x}, \hat{x} \in \mathcal{S}$. Denote the multiplication operator $M_{x}$ by

$$
\left(M_{x} \psi\right)(\hat{x}):=e^{-i \kappa \hat{x} \cdot x} \psi(\hat{x}), \quad \hat{x} \in \mathcal{S} .
$$

The adjoint of $M_{x}$ with respect to the $L^{2}$ scalar product is given by

$$
\left(M_{x}^{*} \psi\right)(\hat{x}):=e^{i \kappa \hat{x} \cdot x} \psi(\hat{x}), \quad \hat{x} \in \mathcal{S} .
$$

From this obtain

$$
S_{\partial G(x)}^{\infty}=M_{x} S_{\partial G_{0}^{\prime}}^{\infty} \quad S_{\partial G(x)}^{\infty, *}=S_{\partial G_{0}}^{\infty, *} M_{x}^{*}
$$

and

$$
R_{\alpha}=R_{0, \alpha} M_{x}^{*}
$$

For this particular setting where translations can be used equation (4.8) speeds-up the calculation of equations (4.2) and (4.3) by a large factor depending on the number of test domains which are used. Numerical examples for the range test are shown in Section 5.3. This speed-up is used for all methods under consideration in the part for acoustic scattering.

\subsection{Potential method}

First, the original potential method of Kirsch and Kress (1986) (see [19], [20], [21] and [8]) is introduced here. Then the range test is used to formulate a new version of the potential method with improved convergence properties.

For the potential method the inverse problem at hand is given in the following definition.

Definition 4.2.1 (Acoustic inverse shape reconstruction). In the setting of the acoustic scattering (Definition 3.0.7) in the dimension $m=2$ the following is given: 
- one incident plane wave $u^{i}$ from the direction $d \in \mathrm{S}$.

- an aperture of the measurement circle $\Lambda \subset \mathcal{S}$.

- the measured far field pattern $u^{\infty}$ on $\Lambda$.

- the boundary condition on the unknown obstacle $D$.

Then find the shape of the unknown boundary of $D$.

The basic idea of the potential method of Kirsch and Kress is to search for the scattered field $u^{s}$ in the form of a single-layer potential (2.21) $S_{\partial G}$. Given the measured far field pattern $u^{\infty}(\cdot, d)$ solve the integral equation (4.1), i.e. the same integral equation as for the range test (Section 4.1) but which now have the property that the test domain $G$ is a subset of $D$. The scattered field is calculated by $u^{s}=\left(S_{\partial G} \varphi\right)$. Then, the shape of the scatterer $D$ is determined via a nonlinear optimisation problem using the boundary condition for the total field $u$. The setup and idea is shown in a) of Figure 4.2.

With the approximate solution $\varphi_{\alpha}=R_{\alpha} u^{\infty}$ by (4.2) it is possible to obtain an approximation $u_{\alpha}^{s}$ to the the scattered field $u^{s}$ on $\mathbb{R}^{m} \backslash \bar{G}$ and its extension into the interior $D \backslash \bar{G}$ by

$$
u_{\alpha}^{s}(x, d)=S_{\partial G}\left(R_{\alpha} u^{\infty}(\cdot, d)\right)(x)
$$

For convergence of the solution of the integral equation (4.1) it is a important condition that the analytic extension of $u^{s}$ into $\mathbb{R}^{m} \backslash \bar{G}$ is possible. To obtain convergence for shape reconstruction when the extensibility condition is violated (which is the case for many situations) the calculation of $u^{s}$ and the search for the unknown boundary has been combined into a nonliner optimisation problem [8, Section 5.4].

\subsubsection{Modification using the range test}

A modified version of the Kirsch-Kress method can be obtained with the help of the range test (Section 4.1). Then, the reconstruction problem can be split into a linear ill-posed part for the of reconstruction $u^{\mathcal{S}}$ and a nonlinear but well-posed problem to find the shape $\partial D$. Further, the test domains $G$ are now bigger than $D$. For the convergence the convergence properties of the range test are used, i.e. the reconstruction converges in the case where $u^{s}$ can be analytically extended into $\mathbb{R}^{m} \backslash \bar{G}$. The resulting setup is shown b) of Figure 4.2.

Algorithm 2 (Modified Kirsch-Kress method.). First, select a finite set of test domains $G^{(j)} \subset \mathbb{R}^{m}$ for $j \in \mathcal{J}$ with some index set $\mathcal{J}$. Further, assume that the $G^{(j)}$ satisfy

$$
D=\bigcap_{D \subset G^{(j)}} G^{(j)}
$$

For each test domain $G^{(j)}$ use the range test to evaluate whether the field $u^{\infty}$ can be analytically extended into the exterior of $\overline{G^{(j)}}$. In this case $G^{(j)}$ is called positive. If $G^{(j)}$ is 

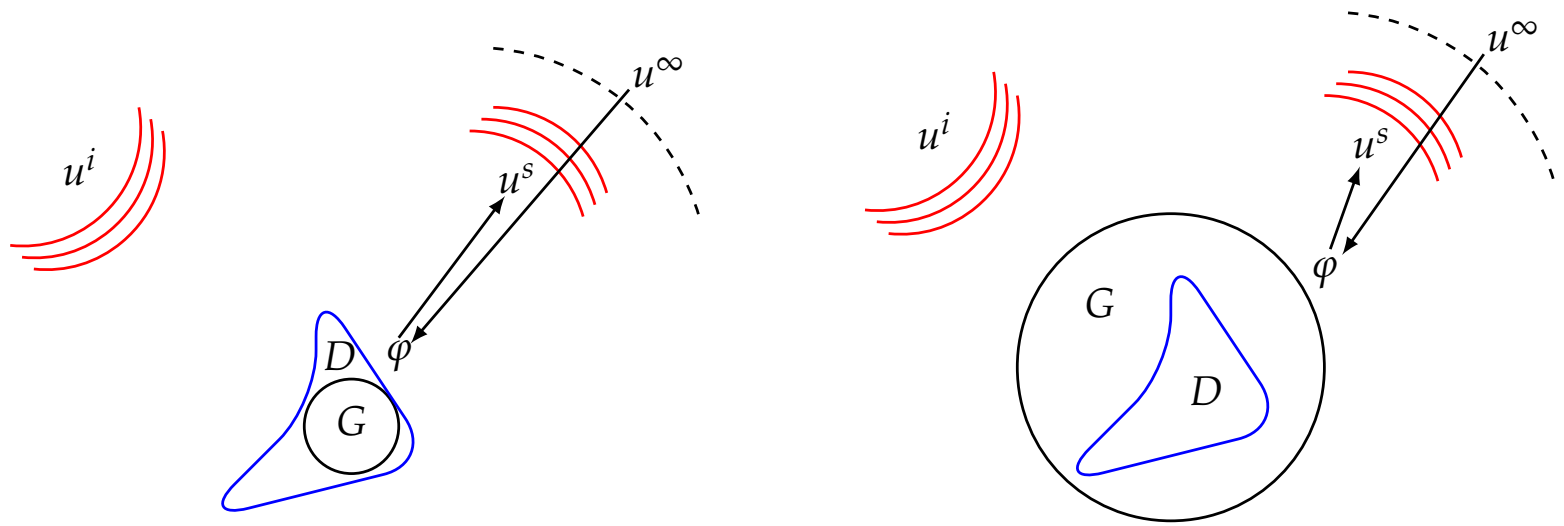

Figure 4.2: Setup and idea of the potential method a) and the modified potential method $\mathrm{b}$ ). The idea is to get the density $\varphi$ from the measured $u^{\infty}$ and from that the scattered field $u^{s}$. The main difference in the setup between the two methods is the size of the test domain $G$.

positive, use the evaluation of the single-layer potential to calculate an approximation $u_{\alpha}^{s, j}$ of the scattered field $u^{s}$ on $\mathbb{R}^{m} \backslash \overline{G^{(j)}}$.

Then, in a second step, combine all reconstructions $u_{\alpha}^{s, j}$ on $\mathbb{R}^{m} \backslash \overline{G^{(j)}}$ for positive test domains $G^{(j)}$ to construct a field $u_{\alpha}^{s}$ on an open set $M \supset \mathbb{R}^{m} \backslash \bar{D}$ as follows. Employ the characteristic function

$$
\chi_{B}(x):= \begin{cases}1, & \text { if } x \in B \\ 0, & \text { otherwise }\end{cases}
$$

and

$$
\sigma(x):=\sum_{G^{(j)}} \chi_{\text {positive }} \chi_{\mathbb{R}^{m} \backslash \overline{G^{(j)}}(x),}
$$

which is well defined for a finite number of test domains. These functions are used for the definition of the reconstruction of the scattered field $u_{\alpha}^{s}$ as

$$
u_{\alpha}^{s}:=\frac{1}{\sigma(x)} \sum_{G^{(j)}} \chi_{\mathbb{R}^{m} \backslash \overline{G^{(j)}}}(x) u_{\alpha}^{s, j}(x)
$$

In the third step search for the shape of $D$ using the Dirichlet or Neumann boundary condition on $\partial D \subset \bar{M}$.

Numerical examples for the modified Kirsch-Kress method will be presented in Section 5.4. For the Kirsch-Kress method the efficient translations of the test domains $G^{(j)}$ as in the range test (4.6) also can be used in the same way. The next steps are completing the analysis of the modified Kirsch-Kress method and showing that the method converges.

Note that the modified Kirsch-Kress method avoids the nonlinear optimisation approach for the ill-posed part and truly splits the reconstruction problem into a linear ill-posed part to reconstruction $u^{s}$ and a nonlinear but well-posed part to find the shape $\partial D$. Here, due to the use of the range test and the use of a set of test 
domains $G^{(j)}$ convergence can be obtained for the reconstruction on the set $M$. Thus, convergence can be also obtained for $u_{\alpha}^{s}$ towards a true scattered field $u^{s}$ of the inverse problem. This result will be formulated in the following theorem.

Theorem 4.2.2 (Convergence of modified Kirsch-Kress method.). Assume that the (finite) set of test domains $G^{(j)}, j \in \mathcal{J}$ is sufficiently rich such that equation (4.10) is satisfied. Then, the modified Kirsch-Kress method generates a set

$$
\tilde{M}:=\bigcap_{G^{(j)} \text { positive }} \overline{G^{(j)}}
$$

which is a closed subset of $\bar{D}$. As a consequence, the open set $M:=\tilde{M}^{c}$ contains $\mathbb{R}^{m} \backslash \bar{D}$. For true data $u^{\infty}$ the method calculates an approximation $u_{\alpha}^{s}$ to $u^{s}$ with

$$
u_{\alpha}^{s}(x) \rightarrow u^{s}(x), \quad \alpha \rightarrow 0
$$

for $x \in M$.

Proof. If $D \subset G^{(j)}$ then equation (4.1) is solvable, thus $G^{(j)}$ is positive. As the set of test domains is sufficiently rich and the extension of $u^{s}$ always contains $\mathbb{R}^{m} \backslash D$, the intersection $\tilde{M}$ of all positive test domains $G^{(j)}$ is a subset of the scatterer $D$. Then, the complement $\mathbb{R}^{m} \backslash \bar{D}$ of $\bar{D}$ is a subset of $M=\tilde{M}^{c}$, i.e. $M$ contains $\mathbb{R}^{m} \backslash \bar{D}$. For all positive $G^{(j)}$ following the range test (Theorem 3.5 in [41]) the density $\varphi_{\alpha}$ converges to the true solution $\varphi$ of (4.1) for $\alpha \rightarrow 0$ and thus $u_{\alpha}^{s, j}(x)$ tends to $u^{s}(x)$ for $\alpha \rightarrow 0$ for each fixed $x \in \mathbb{R}^{m} \backslash \overline{G^{(j)}}$. Then, from equation (4.11) it holds that

$$
\begin{aligned}
u_{\alpha}^{s} & =\frac{1}{\sigma(x)} \sum_{G^{(j)}} \chi_{\text {positive }} \chi_{\mathbb{R}^{m} \backslash \overline{G^{(j)}}}(x) u_{\alpha}^{s, j}(x) \\
& \rightarrow \frac{1}{\sigma(x)} \sum_{G^{(j)}} \chi_{\mathbb{R}^{m} \backslash \overline{G^{(j)}}}(x) u^{s}(x) \\
& =u^{s}(x) \frac{1}{\sigma(x)} \sum_{G^{(j)}} \chi_{\text {positive }} \chi_{\mathbb{R}^{m} \backslash \overline{G^{(j)}}}(x) \\
& =u^{s}(x),
\end{aligned}
$$

in the limit $\alpha \rightarrow 0, x \in M$. Thus, the convergence of the field reconstruction (4.13) is shown.

\subsection{Multiwave range test}

The range test can be extended to the situation of several incident plane waves of one fixed frequency, which is then called the multiwave range test. This extension enables reconstructions of the full shape of $D$ rather than a subset.

For the multiwave range test the inverse problem is given in the following definition. 
Definition 4.3.1 (Acoustic inverse shape reconstruction). In the setting of the acoustic scattering (Definition 3.0.7) in the dimension $m=2$ the following is given:

- many incident plane waves $u^{i}(\cdot, d)$ from directions $d \in \mathbb{S}$.

- an aperture of the measurement circle $\Lambda \subset \mathcal{S}$.

- the measured far field patterns $u^{\infty}(\cdot, d)$ for all $d \in \Lambda$.

Then find the shape of the unknown boundary of $D$.

If the far field pattern is given for several incident waves, then first use the onewave range test for each plane wave. If it fails for one wave, then the test domain $G$ can be marked negative. Assume that the field $u^{s}$ is analytic extensible into $\mathbb{R}^{m} \backslash \bar{G}$, i.e. the equation (4.1) is solvable for all directions $d \subset \Lambda$. In other words: the domain $G$ is positive for all one-wave range tests applied to the far field pattern $u^{\infty}(\cdot, d), d \in \Lambda$. In this case an approximation for $u^{S}(\cdot, d)$ for $d \in \Lambda$ can be calculated by (4.9). Now use the mixed reciprocity relation (Theorem 3.0.9) in the form

$$
u^{s}(x,-d)=\frac{1}{\gamma_{m}} \Phi^{\infty}(d, x),
$$

where $\Phi^{\infty}$ is the far field pattern of the scattered wave from a point source. With the definition

$$
v_{\alpha}(d, x):=\gamma_{m} S_{\partial G}\left(R_{\alpha} u^{\infty}(\cdot,-d)\right)(x)
$$

for $d \in \mathrm{S}$ and $x \in \mathbb{R}^{m} \backslash \bar{G}$ the approximation

$$
\Phi^{\infty}(d, x) \approx v_{\alpha}(d, x)
$$

is obtained.

Given the far field pattern $\Phi^{\infty}(\cdot, x)$ or its approximation $v_{\alpha}(\cdot, x)$ respectively for fixed $x$ on the set $S$, the extensibility of this field into the exterior of $\mathbb{R}^{m} \backslash \bar{G}$ can now be tested in the same way as in the range test for one wave above, i.e. the range test is repeated, but now applied to $v_{\alpha}(\cdot, x)$ as far field pattern. This is carried out by solving the equation

$$
S_{\partial G}^{\infty} \psi_{x}=v_{\alpha}(\cdot, x), \quad \forall x \in \mathbb{R}^{m} \backslash \bar{G} .
$$

If the scattered field of a point source $\Phi^{S}(\cdot, x)$ can be analytically extended into $\mathbb{R}^{m} \backslash \bar{G}$, then the equation

$$
S_{\partial G}^{\infty} \psi_{x}=\Phi^{\infty}(d, x)
$$

- which is approximated by (4.16) - can be solved. If the equation (4.17) can not be solved, then the field $\Phi^{\infty}(\cdot, x)$ cannot be analytically extended into $\mathbb{R}^{m} \backslash \bar{G}$. However, if $D \not \subset G$, i.e. consider a sequence of points $x_{n} \subset \mathbb{R}^{m} \backslash \bar{G}$ with $x_{n} \rightarrow x_{0}$ for $n \rightarrow \infty$ and $x_{0} \in \partial D$ with $x_{0} \notin \bar{G}$. Then for $n \rightarrow \infty$ the field $\Phi^{S}\left(x_{n}, x_{n}\right)$ becomes singular ([35, Theorem 2.1.15]) and the norm of the solution $\psi_{x_{n}}$ of equation (4.16) cannot be uniformly bounded. The idea and basic setup for this method is shown in Figure 4.3.

With the following theorem a precise formulation for the extensibility of the field $\Phi^{s}(\cdot, x)$ into the interior of $G$ is given. 


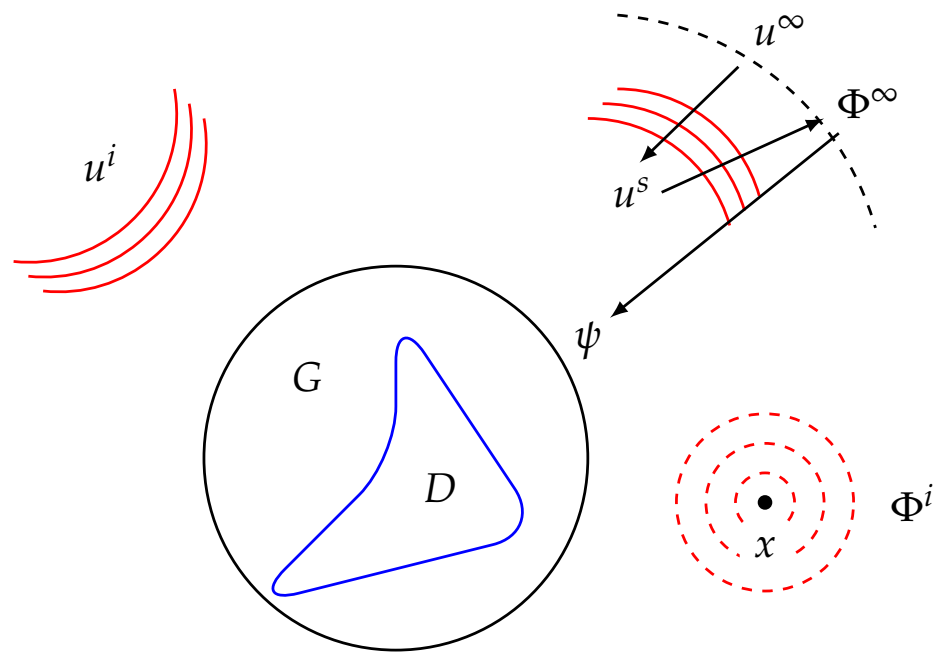

Figure 4.3: Idea and setup of the multiwave range test. From the measured data $u^{\infty}$ the scattered field for one wave $u_{1}^{s}$ is reconstructed. Via the mixed reciprocity relation the far field pattern $\Phi^{\infty}$ from a point source in $x$ is established. Then calculate the density which defines $\Phi^{S}(\cdot, x)$.

Theorem 4.3.2 (Extensibility properties). Assume that $\bar{D} \subset G$. Then the field $\Phi^{s}(\cdot, x)$ can be analytically extended up to $\mathbb{R}^{m} \backslash \bar{G}$ uniformly for all $x \in \mathbb{R}^{m} \backslash \bar{G}$, i.e. the $L^{2}$-norms of the densities $\psi_{x}$ solving (4.17) on $\partial G$ are uniformly bounded for $x \in \mathbb{R}^{m} \backslash \bar{G}$.

If $D \not \subset G$, then the fields $\Phi^{s}(\cdot, x)$ cannot be extended up to $\mathbb{R}^{m} \backslash \bar{G}$ uniformly for all $x \in \mathbb{R}^{m} \backslash \bar{G}$, i.e. the extensions will not be uniform in the sense that the $L^{2}$-norms of the densities $\psi_{x}$ solving (4.17) on $\partial G$ will not be uniformly bounded for $x \in \mathbb{R}^{m} \backslash \bar{G}$.

Proof. case $\bar{D} \subset \mathrm{G}$ : By definition of $\Phi^{s}(\cdot, x)$ as the scattered field for scattering of $\Phi(\cdot, x)$ by $D$, the field is defined in $\mathbb{R}^{m} \backslash D$. The $L^{2}$-norm of $\Phi^{S}(\cdot, x)$ is uniformly bounded for all $x \in \mathbb{R}^{m} \backslash \bar{G}$, since the boundary values $\left.\Phi(\cdot, x)\right|_{\partial D}$ for $x \in \mathbb{R}^{m} \backslash \bar{G}$ are uniformly bounded in $C(\partial D)$ and the mapping of the boundary values onto the scattered field on $\partial G \subset \mathbb{R}^{m} \backslash \bar{D}$ is bounded in any norm $C^{2}(\partial G)$. Since the scattered field $\Phi^{S}(\cdot, x)$ is bounded, the corresponding density $\psi_{x}$ is bounded in the $L^{2}$ sense.

case $D \not \subset G$ : For a proof by contradiction assume that the fields $\Phi^{s}(\cdot, x)$ can be analytically extended up to $\mathbb{R}^{m} \backslash \bar{G}$ such that the $L^{2}$-norms of the fields on $\partial G$ are uniformly bounded for $x \in \mathbb{R}^{m} \backslash \bar{G}$. Let $x_{0} \in \partial D$ be a point with $x_{0} \subset \mathbb{R}^{m} \backslash \bar{G}$. Consider a sequence of points $x_{n} \subset \mathbb{R}^{m} \backslash \bar{G}$ with $x_{n} \rightarrow x_{0}$ for $n \rightarrow \infty$. Then, $\Phi^{s}\left(\cdot, x_{n}\right)$ can be represented as a solution to the exterior Dirichlet problem in $\mathbb{R}^{m} \backslash G$ with boundary values which are uniformly bounded for all $n \in \mathbb{N}$. Thus, there is a constant $C>0$ such that

$$
\left|\Phi^{S}\left(x_{n}, x_{n}\right)\right| \leq C, \quad n \in \mathbb{N},
$$

However, by the Theorem 2.1.15 of [35] for the Dirichlet and Neumann boundary condition the estimate

$$
\left|\Phi^{s}\left(x_{n}, x_{n}\right)\right| \rightarrow \infty, n \rightarrow \infty .
$$

holds which contradicts (4.18). Thus, the assumption cannot be valid and the fields are not analytically extensible uniformly for $x \in \mathbb{R}^{m} \backslash \bar{G}$. 
Remark. This implies that if all densities $\psi_{x}$ are uniformly bounded for $x \in \mathbb{R}^{m} \backslash \bar{G}$, i.e.

$$
\mu(G):=\sup _{x \in \mathcal{G}}\left\|\psi_{x}\right\|_{L^{2}(\partial G)} \leq C
$$

with some sampling grid $\mathcal{G} \subset \mathbb{R}^{m} \backslash \bar{G}$ and an appropriately chosen constant $C$, it can be concluded that $\bar{D} \subset G$ and $D \not \subset G$ otherwise.

Now, using a set of test domains $G^{(j)}$ in the domain of observation with the index $j \in \mathcal{J}$ and index set $\mathcal{J}$ a reconstruction of $D$ can be obtained by

$$
D_{r e c}:=\bigcap_{j \in \mathcal{J} \text { s.th. } \mu\left(G^{(j)}\right) \leq C} G^{(j)} .
$$

With an proper choice of this family of test domains in principle full reconstructions of the unknown scatterer $D$ can be obtained.

The numerical calculation of the densities $\psi_{x}$ for all $x \in \mathcal{G}$ needs a big effort. To reduce this effort an efficient implementation of this test will be described in the next paragraph.

Efficiency and visualisation. First, the same efficient calculations through translating the test domains $G^{(j)}$ can be used as in the range test (4.6).

Second, in equation (4.19) the evaluation for $\psi_{x}$ is for all $x \in \mathcal{G}$, but this is not necessary for the realization of the method. The following technique (see [39] for a comparison with the above set handling approach) is used to recover the unknown domain.

First, consider an admissible test domain $G_{0}$ such that $0 \notin \overline{G_{0}}$. Usually $G_{0}$ has to be chosen such that $d\left(0, G_{0}\right) \ll 1$. Then, use the translations (4.5) for $x \in M, M \subset \mathbb{R}^{m}$, the domain of observation. The set of domains

$$
\Theta:=\{G(x): x \in M\}
$$

is called a configuration $\Theta$ of the point $x$ and the test domain $G(x)$. A configuration is determined by the choice of $G_{0}$.

For the source point $x$ and the domain $G(x)$ the multiwave range test according to (4.15) and (4.16) is used to calculate a density $\psi_{x}$. The norm $\left\|\psi_{x}\right\|$ is then attributed to the point $x \in M$. Due to the construction of the approximations the norm of $\psi_{x}$ becomes large if $x \rightarrow \partial D$ and $D \subset G(x)$. Furthermore, the norm $\left\|\psi_{x}\right\|$ is large when $D$ is not a subset of $G(x)$. It will be small when $x$ is in the exterior of $\bar{D}$ such that $\bar{D} \subset G(x)$. Thus, the set $M$ where $\psi_{x}$ has small norm, is a subset of the exterior of $D$, compare Figure 4.4 and Figure 4.5 for visualisation of this description. The decision whether $\left\|\psi_{x}\right\|$ is small or large is carried out using a cut-off constant $C$. The sets $B_{j}:=M^{c}$ are calculated for several different configurations $\Theta_{j}, j=1, \ldots, n$. Then the intersections

$$
D_{\text {approx }}:=\bigcap_{j=1, \ldots, n} B_{j}
$$




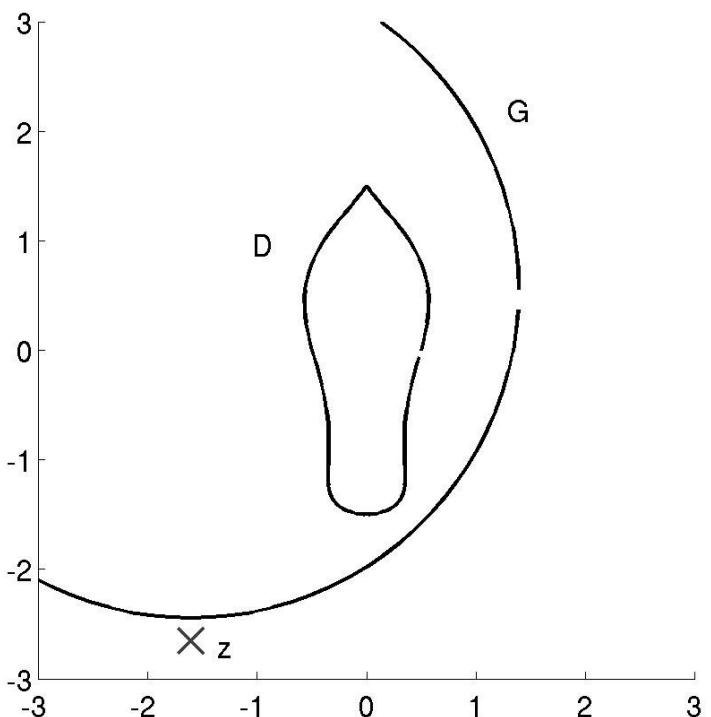

(a) Configuration

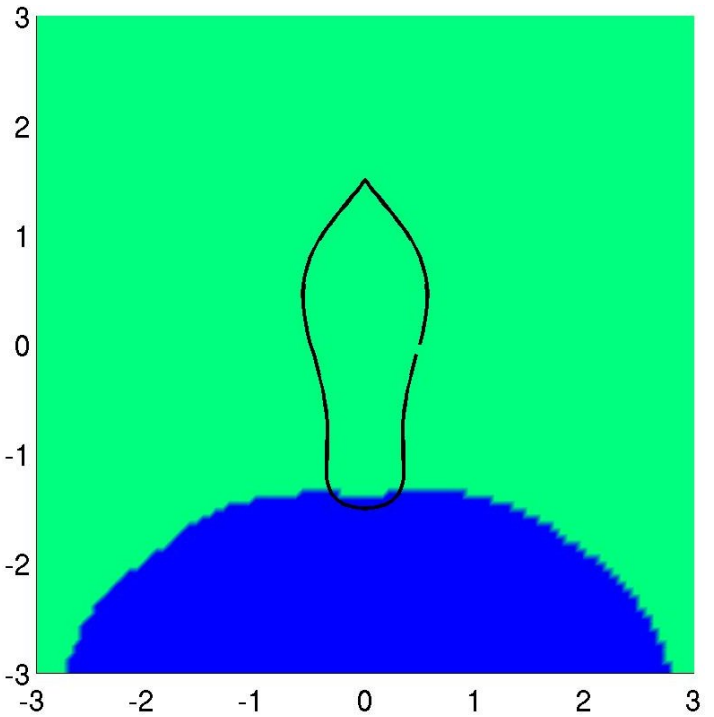

(b) Enlightened area

Figure 4.4: Configuration and area of small $\left\|\psi_{x}\right\|$ in blue (Step 2 of algorithm 3). The test domain is a circle. The evaluation point lies $z$ below the test domain.

of the sets $B_{j}$ are used to calculate an approximation to the unknown scatterer $D$.

A detailed computational version of the multiwave range test is given in the following algorithm.

Algorithm 3 (Multiwave range test). Let $M$ be some area in $\mathbb{R}^{m}$ where the search of the unknown scatterer $D$ takes place. The set of directions of incident plane waves is denoted by $-\Lambda \subset \mathrm{S}$. Let the far field pattern $u^{\infty}(\cdot, d) \in L^{2}(\Lambda)$ be given for all $d \in-\Lambda$. The multiwave range test calculates a reconstruction to the unknown scatterer $D$ by the following steps.

1. A sampling grid $\mathcal{M}$ in $M$ is chosen. Then build up a number of configurations $\Theta_{j}$ for $j=1, \ldots, n$ by construction of a set of admissible reference test domains $G_{0}^{(j)}$ with boundary of class $C^{2}$, such that the homogeneous interior Dirichlet problem for $G_{0}^{(j)}$ das only the trivial solution and $0 \notin \overline{G_{0}^{(j)}}$. Further, $G_{0}^{(j)}$ is chosen such that $\rho:=d\left(0, G_{0}^{(j)}\right)>0$ is sufficiently small. For each $j=1, \ldots, n$ carry out the following steps.

a) Set up the operator $S_{\partial G_{0}^{(j)}}^{\infty}: L^{2}\left(\partial G_{0}\right) \mapsto L^{2}(\Lambda)$ defined in (2.23) and with some $\alpha>0$ calculate the regularised inverse

$$
R_{0, \alpha}^{(j)}=\left(\alpha I+S_{\partial G_{0}^{(j)}}^{\infty, *} S_{\partial G_{0}^{(j)}}^{\infty}\right)^{-1} S_{\partial G_{0}^{(j)}}^{\infty, *} .
$$

b) Set up the single-layer evaluation operator

$$
S_{\text {eval }}: L^{2}\left(\partial G_{0}\right) \mapsto \mathbb{C}, \quad S_{\text {eval }} \phi:=\int_{\partial G_{0}} \Phi(0, y) \phi(y) \mathrm{d} s(y)
$$




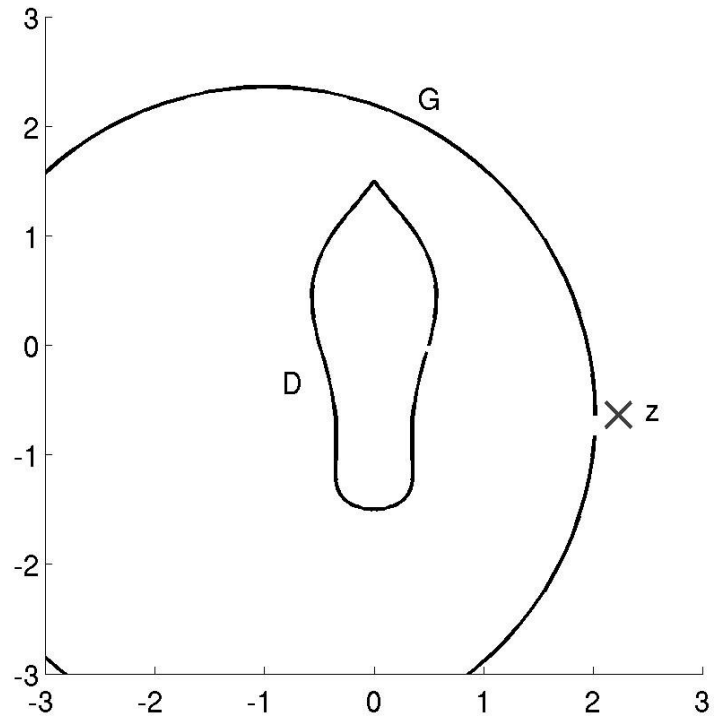

(a) Configuration

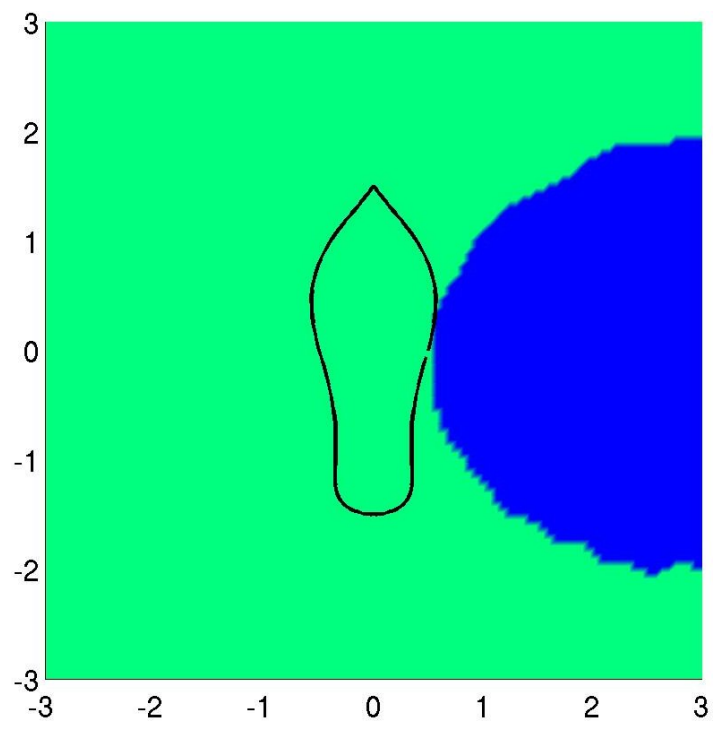

(b) Enlightened area

Figure 4.5: Configuration and area of small $\left\|\psi_{x}\right\|$ in blue (Step 2 of algorithm 3). The test domain is a circle. The evaluation point is on the right-hand side of the test domain.

and calculate

$$
W_{\alpha}^{(j)}:=S_{e v a l} R_{0, \alpha}^{(j)}
$$

c) For each $x \in \mathcal{M}$ calculate

$$
v^{(j)}(d, x):=\gamma_{m} W_{\alpha}^{(j)} \overline{M_{x}} u^{\infty}(\cdot,-d), d \in \Lambda
$$

with $M_{x}$ defined in eq. (4.7), as an approximation to $\Phi^{\infty}(d, x)$.

d) For each $x \in \mathcal{M}$ calculate

$$
\psi_{x}^{(j)}:=R_{0, \alpha}^{(j)} \bar{M}_{x} v^{(j)}(\cdot, x)
$$

and

$$
\mu_{j}(x):=\left\|\psi_{x}^{(j)}\right\|_{L^{2}\left(\partial G_{0}\right)} .
$$

2. For chosen cut-off parameter $C$ define the function

$$
b(j, x):= \begin{cases}0, & \text { if } \mu^{(j)}(x) \leq C, \\ 1, & \text { if } \mu^{(j)}(x)>C,\end{cases}
$$

3. Evaluate the minimum of $b(j, \cdot)$ for the configurations $j=1, \ldots, n$ to calculate $a$ reconstruction of the scatterer $D$.

$$
D_{\text {rec }}:=\left\{x \in \mathcal{M}: \min _{j=1, \ldots, n} b(j, x)=1\right\} .
$$

Numerical examples of the multiwave range test are shown in Section 5.5. 


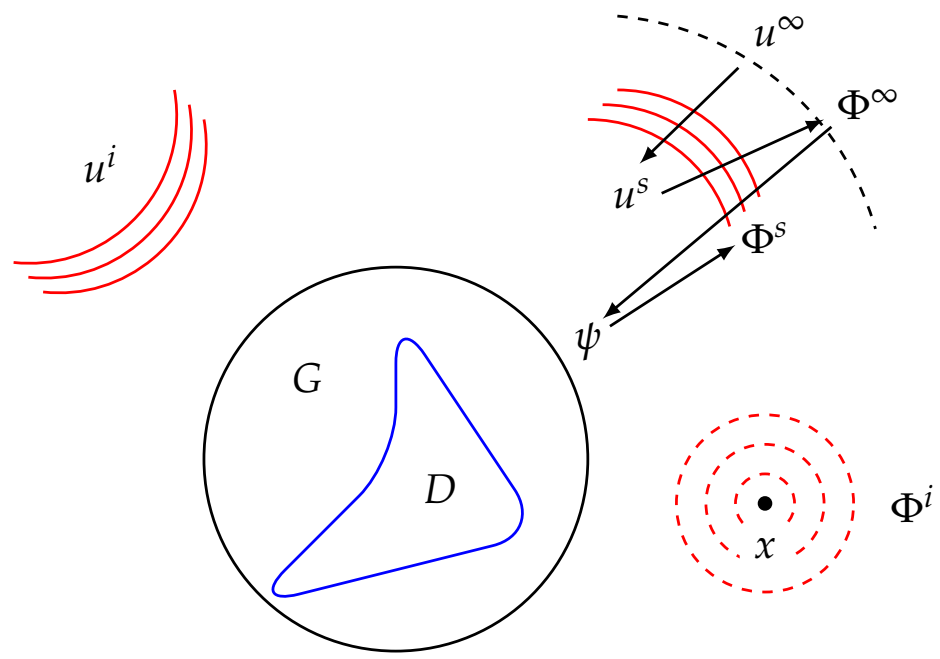

Figure 4.6: Idea and setup of the singular sources method. From the measured data $u^{\infty}$ the scattered field for one wave $u_{1}^{s}$ is reconstructed. Via the mixed reciprocity relation the far field pattern $\Phi^{\infty}$ from a point source in $x$ is established. Then from the density $\psi$ calculate $\Phi^{S}(\cdot, x)$.

\subsection{Singular sources method - alternative}

The chosen inverse problem which is then solved via the singular sources method is given in the following Definition.

Definition 4.4.1 (Acoustic inverse shape reconstruction). In the setting of the acoustic scattering (Definition 3.0.7) in the dimension $m=2$ the following is given:

- many incident plane waves $u^{i}(\cdot, d)$ from directions $d \in \mathrm{S}$.

- an aperture of the measurement circle $\Lambda \subset \mathcal{S}$.

- the measured far field patterns $u^{\infty}(\cdot, d)$ for all $d \in \Lambda$.

Then find the shape of the unknown boundary of $D$.

The idea of the singular sources method is to construct the scattered field $\Phi^{s}(z, z)$ for an incident point source $\Phi(\cdot, z)$ in its source point $z$ and to use the blow-off property

$$
\left|\Phi^{S}(z, z)\right| \rightarrow \infty, \quad z \rightarrow \partial D
$$

to find the unknown shape $\partial D$.

This is an alternative approach to the singular sources method which will use the techniques of the range test. In its original versions [34], [35] the singular sources method is based on the point source method ([37] and [38]), i.e. an application of Green's formula (Theorem B.1.2) and point source approximations, to construct the function $\Phi^{S}(x, z)$. Here the point source method will be replaced by the potential method in combination with the range test to calculate an approximation to the function $\Phi^{S}(z, z)$ 
from the far field patterns $u^{\infty}(\hat{x}, d), \hat{x}, d \in \mathcal{S}$. The analytic continuation of the field $u^{\infty}$ into $u^{s}$ and $\Phi^{\infty}(\cdot, z)$ into $\Phi^{S}(z, z)$ is now accomplished by the potential method.

The first steps follow the equations (4.14) - (4.17) of the multiwave range test. Now, given the field $\Phi^{\infty}(\cdot, x)$ for some $x \in \mathbb{R}^{m} \backslash \bar{G}$ the scattered field

$$
\Phi^{s}(y, x), y, x \in \mathbb{R}^{m} \backslash \bar{G}
$$

can be reconstructed via an evaluation of the single-layer potential (Equation 2.21) with density $\psi_{x}$. For the choice $y=x=z$ an approximation for the function $\Phi^{s}(z, z)$ is obtained, which is known to blow-up when $z$ tends to the unknown boundary. Thus, if there is any intersection $D \cap\left(\mathbb{R}^{m} \backslash \bar{G}\right)$, then it is possible to find a sequence of points $z_{n} \in \mathbb{R}^{m} \backslash \bar{G}$ such that

$$
\lim _{n \rightarrow \infty} z_{n}=z_{*} \in \partial D
$$

As the $\Phi^{s}$ will be calculated using a regularisation with regularisation parameter $\alpha$ it can be denoted as $\Phi_{\alpha}^{s}$ and the following property holds

$$
\Phi_{\alpha}^{S}\left(z_{n}, z_{n}\right) \rightarrow \Phi^{S}\left(z_{n}, z_{n}\right), \quad \alpha \rightarrow 0, n \in \mathbb{N} \text { fixed } .
$$

This leads to: for all constants $C>0$ there exists $n>0$ such that for all $\alpha$ smaller than some fixed $\alpha_{0}>0$ the following holds

$$
\left|\Phi_{\alpha}^{S}\left(z_{n}, z_{n}\right)\right|>C
$$

with chosen $C$. The equation (4.29) is used to find the blow-up and with that the unknown boundary.

The algorithm for the singular sources method can be formulated in nearly the same way as the multiwave range test algorithm. The only change is the calculation of $\mu_{j}(x)$ in step ( $\left.1 \mathrm{~d}\right)$ of Algorithm 3.

Algorithm 4. Following Algorithm 3 and in step (1d) substituting $\left\|\psi_{x}^{(j)}\right\|$ by

$$
\mu_{j}(x):=\left|S_{\text {eval }} \psi_{x}^{(j)}\right|
$$

the algorithm for the alternative version of the singular sources method is obtained.

\subsection{Relation of sampling methods under consideration}

Here the relations between the four methods under consideration, i.e. the range test, the potential method, the multiwave range test and the singular sources method, are compared. Figure 4.7 shows a simple diagram of the relations between the methods. It can be seen, that the one-wave range test is the most simple approach. The potential method may be based on the one-wave range test. Then, the mixed reciprocity relation (Theorem 3.0.9) might be used to formulate a multiwave range test based on the one-wave range test and the potential method. Finally, the multiwave range test can be extended to the singular sources method. 


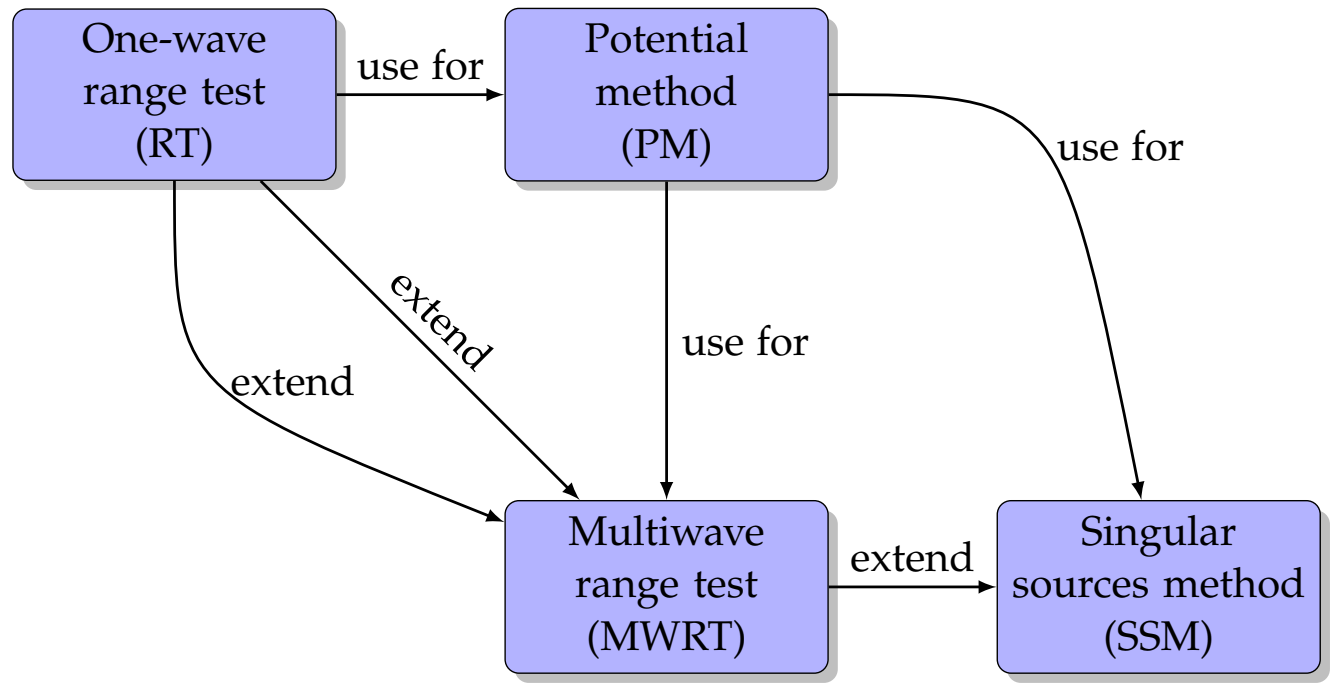

Figure 4.7: The Diagram shows the relation between the one-wave range test, the potential method, the multiwave range test and the singular sources method 
Chapter 4 Inverse problem 


\section{Chapter 5}

\section{Numerics}

The goal of this chapter is the numerical realization of the methods described above in Chapter 4. As a model problem the two-dimensional case is chosen for the numerical proof-of-concept study.

\subsection{Simulation}

First, the discretisation of the boundary $\partial G_{0}$ of some reference domain $G_{0}$ is chosen as $\boldsymbol{y}_{k}, k=1, \ldots, n_{G}$. A uniform discretisation of the unit circle is denoted by $\hat{x}_{j} \in \mathrm{S}$, $j=1, \ldots, n_{S}$ and then define

$$
\Lambda:=\left\{\hat{\boldsymbol{x}}_{j}, j=1, \ldots, n_{S}\right\} .
$$

The far field pattern $u^{\infty}(\hat{x},-d)$ is given for the evaluation points $\hat{x} \in \Lambda$ in all directions of incidence $-d \in \Lambda$, i.e. the matrix

$$
\begin{aligned}
u^{\infty} & =\left(u^{\infty}\left(\hat{\boldsymbol{x}}_{j},-d_{l}\right)\right)_{j, l=1, \ldots, n_{S}} \\
& =\left(\left(u^{\infty}\left(\hat{\boldsymbol{x}}_{j},-d_{1}\right)\right)_{j=1, \ldots, n_{S}}, \ldots,\left(u^{\infty}\left(\hat{\boldsymbol{x}}_{j},-d_{n_{S}}\right)\right)_{j=1, \ldots, n_{S}}\right)
\end{aligned}
$$

is given as data. The $l$-th column of $\boldsymbol{u}^{\infty}$ contains the far field pattern for scattering of plane waves $e^{-i \kappa y \cdot d_{l}}, y \in \mathbb{R}^{m}$ by the scatterer $D$.

A numerical quadrature rule is used for the evaluation of the single-layer boundary operator (2.22) $S_{\partial G}$ and for the calculation of its far field pattern (2.23) $S_{\partial G}^{\infty}$. The application of the trapezoidal quadrature rule (c.f. Kress [23]) to some parameterisation of the curve $\partial G_{0}$ leads to the matrix

$$
S_{\partial G_{0}}^{\infty}:=\gamma_{m}\left(e^{i \kappa \hat{x}_{j} \cdot y_{k}} S_{k}\right)_{j=1, \ldots, n_{S}, k=1, \ldots, n_{G}} .
$$

with weights $s_{k} \in \mathbb{R}$. Now, for the stabilised inversion of $S_{\partial G_{0}}^{\infty}$ Tikhonov regularization (Theorem A.4.3) is used, i.e. calculate

$$
\boldsymbol{R}_{0, \alpha}:=\left(\alpha \boldsymbol{I}+\boldsymbol{S}_{\partial G_{0}}^{\infty, *} S_{\partial G_{0}}^{\infty}\right)^{-1} S_{\partial G_{0}}^{\infty, *}
$$

for $\alpha>0$. Here $*$ denotes the complex conjugate transposed matrix and $I$ is the identity matrix. 


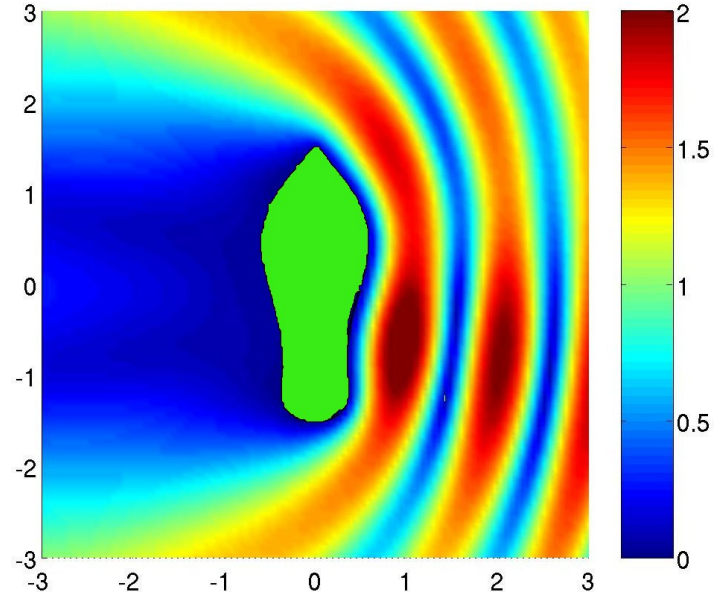

(a) Dirichlet

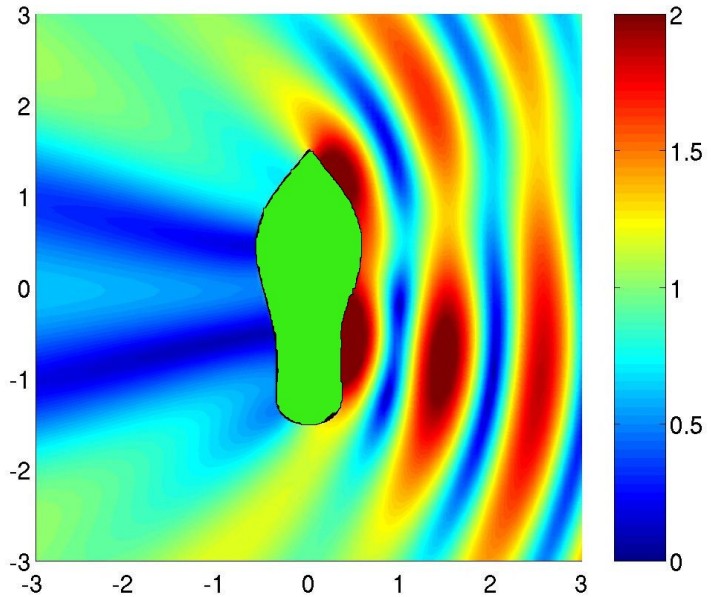

(b) Neumann

Figure 5.1: Illustration of the total field from calculating the direct scattering problem for a boat-like scatterer. The incident plane wave is coming from the right with wave number $\kappa=3$.

The single-layer potential evaluation operator $S_{\text {eval }}$ is represented by a row vector

$$
S_{\text {eval }}=\left(\boldsymbol{\Phi}\left(0, y_{k}\right) s_{k}\right)_{k=1, \ldots, n_{G}}
$$

and the potential is evaluated by

$$
\boldsymbol{S}_{\mathrm{eval}} \circ \boldsymbol{\varphi}=\sum_{k=1, \ldots, n_{G}} \boldsymbol{\Phi}\left(0, y_{k}\right) s_{k} \varphi_{k}
$$

for some column vector $\varphi$. The multiplication operator $M_{x}$ defined in (4.7) is discretized by the diagonal matrix

$$
\boldsymbol{M}_{x}:=\operatorname{diag}\left(e^{-i \kappa \hat{x}_{j} \cdot x}\right)_{j=1, \ldots, n_{S}} .
$$

The reconstruction operator $R_{\alpha}$ for translated domains $G(x)$ is now represented by

$$
\boldsymbol{R}_{\alpha}=\boldsymbol{R}_{0, \alpha} \overline{\boldsymbol{M}_{x}} .
$$

As a basis for later comparisons of the reconstruction schemes, the numerical results for the direct problem are shown in Figure 5.1. The images show the modulus of the total field with an incoming plane wave from the right hand side for the case of the Dirichlet boundary condition in a) of Figure 5.1 and for the Neumann boundary conditions in b) of Figure 5.1.

\subsection{Choice of the test domains and regularisation parameters}

In principle, the choice of test domains $G^{(j)}$ has no limits for all methods under consideration. To obtain positive test domains (in the sense of Algorithm 1 ) the test 
domains has to be chosen sufficiently large such that the scattered field can be analytically extended into their exterior, i.e. the test domains have to be large enough to be in principle able to contain the convex scattering support.

For simple settings as considered in this work, it is sufficient to work with simple test domains. Here, the choice of test domains are circles and the scan of the area with the unknown domain was done by translations of one fixed circle.

For more complex situations the test domains needs to be no longer convex. This might lead to difficult algorithmical questions, which are not covered in this work. For complex situations, the choice carried out in this work can be seen as a first step of a multistep algorithm (see also [35]) where in the first step the convex hull of the scatterers under consideration are reconstructed and in a second step a more involved choice of the test domains is used.

Both the regularisation parameter $\alpha$ and the cut-off constant $C$ need to be adapted to the particular choice of the test domain under consideration. This problem applies to all sampling methods, compare the survey article [36]. The choice of these parameters can be carried out for example by simulation using a reference setting. Here, the parameters were chosen by trial and error, which is considered to be legitimate within a principle feasibility study for a new method. For the reconstructions, data from the numerical simulations are used, which has approximately $1-2 \%$ numerical error. Reconstructions of the singular sources method (or probe method) with different random errors can be found in [11], Figure 6.

The behaviour of reconstructions for different choices of $C$ for a typical sampling method is shown in Figure 6 of [27]. If $C$ is chosen too large, the reconstruction is too small. If $C$ is chosen smaller, then the reconstruction becomes larger.

\subsection{Range test}

Here, the range test for one wave is studied in its numerical setting. As described in theory of the range test (Section 4.1), the exact shape of the scatterer cannot be reconstructed. However, it is still possible to obtain some reconstruction which reflects basic properties of the shape of the scatterer under consideration.

The translation technique (Theorem 4.1.7) is used to speed up the calculations, where for simplicity a circle of radius $R=4$ with center $x_{0}=(0,0)$ is used as reference test domain $G_{0}$. In this case the density is calculated via

$$
\boldsymbol{\varphi}_{x}(d):=\boldsymbol{R}_{0, \alpha} \overline{\boldsymbol{M}_{x}} \circ \boldsymbol{u}^{\infty}(d)
$$

for all test domains $G(x)$ and for one far field pattern $\boldsymbol{u}^{\infty}(d)$. Here, the dependence on $\alpha$ is kept fixed with the value $\alpha=10^{-9}$ for the calculations. The value of $x$ is chosen appropriately such that the center of $G(x)$ takes values on a grid covering $M=[-3,3] \times[-3,3]$. Then the functional $\mu(4.4)$ can be written as

$$
\boldsymbol{\mu}(x, d)=\sqrt{\boldsymbol{\varphi}_{x}^{T}(d) \cdot \boldsymbol{\varphi}_{x}(d)} .
$$

The size of the real number $\mu(x, d)$ is an indicator for the extensibility of the field $u^{\infty}(d)$ into the exterior of the test domain $G(x)$. 


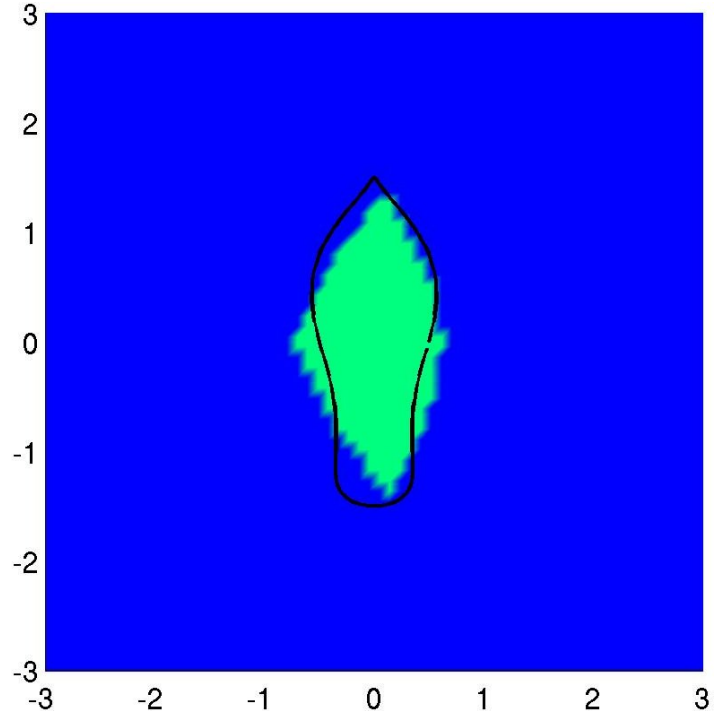

(a) Dirichlet boundary condition

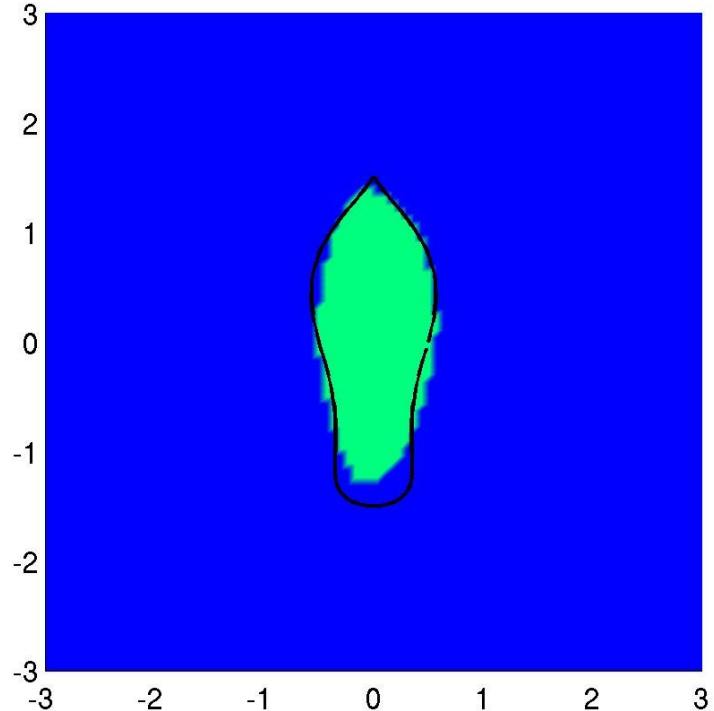

(b) Neumann boundary condition

Figure 5.2: Reconstruction of the shape with the range test with one incident plane wave from the right with wave number $\kappa=3$.

Numerically, a cut-off parameter is needed to decide whether the test domain is positive or negative. The cut-off parameter $C$ can be determined by calibration: use a simulated far field pattern for a circle, carry out the reconstruction and choose $C$ appropriately to achieve reasonable reconstructions for the circle. Then, use the same constant $C$ for other domains as well.

The reconstruction of the shape of a boat-like scatterer with one incoming plane wave from the right can be seen in Figure 5.2. The wave number $\kappa$ is 3 .

\subsection{Modified potential method}

Here, the steps of Algorithm 2 for the modified potential method are followed. First, the range test is used to test whether the analytic continuation lies within a given test domain. Then, calculate $\psi_{x}(d)$ via (5.2) and determine $\mu(x, d)$ by (5.3). With given cut-off constant $C$ the test domain is evaluated for positiveness and for every positive test domain an approximation to the scattered field $u^{S}$ is calculated. For a point $z \in \mathbb{R}^{m} \backslash G^{(j)}$ the fields $u^{S}\left(z,-d_{1}\right), \ldots, u^{S}\left(z,-d_{n_{S}}\right)$ are approximated by the row vector

$$
\boldsymbol{u}_{\alpha}^{S}(z):=S_{\partial G, z} \circ \boldsymbol{R}_{\alpha} \circ \boldsymbol{u}^{\infty},
$$

with $\boldsymbol{u}^{\infty}$ defined in (5.1) and the row vector $S_{\partial G, z}$ defined as

$$
S_{\partial G, z}=\left(\Phi\left(z, y_{k}\right) s_{k}\right)_{k=1, \ldots, n_{G}} .
$$

For the later use in the multiwave range test as the Kirsch-Kress reconstruction step, the calculation of the scattered field here is simultaneously carried out for $n_{s}$ far field 


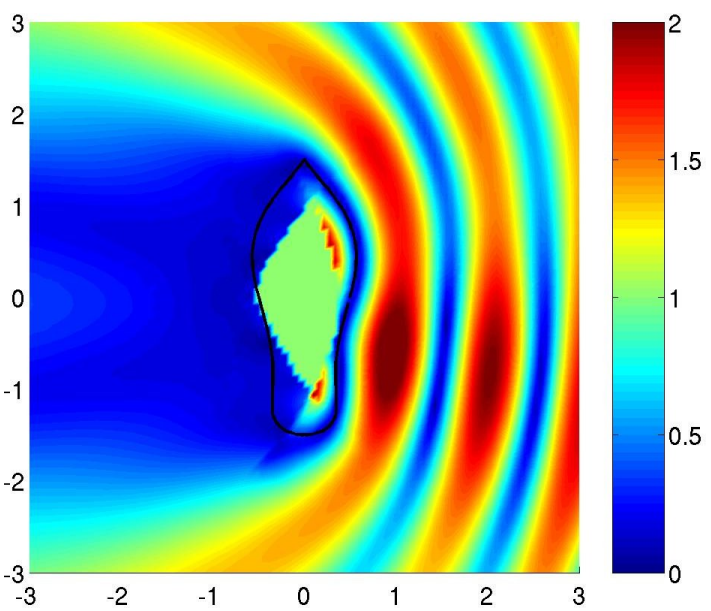

(a) Reconstructed total field

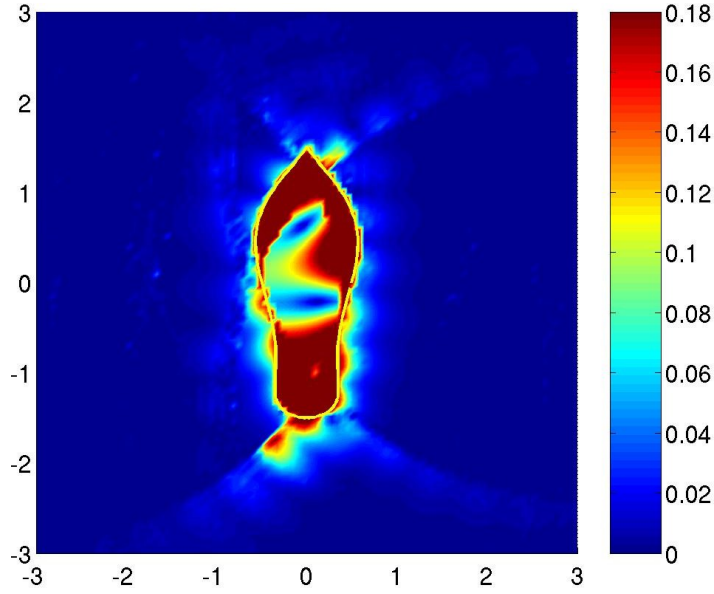

(b) Difference to the true solution

Figure 5.3: Field reconstructions with the modified method of Kirsch-Kress in the case of Dirichlet boundary conditions for a boat-like scatterer with an incident plane wave coming from the right with $\kappa=3$. The test domains have a radius of 4 .

patterns, or one if its the potential method itself. A simultaneous evaluation of the functional for different $z \in \mathbb{R}^{m} \backslash \bar{G}$ can be obtained by definition of the matrix

$$
S_{\partial G}:=\left(S_{\partial G, z_{l}}\right)_{l=1, \ldots, n_{l}} .
$$

with $n_{l}$ points in $M$. Then, in (5.4) $S_{\partial G, z}$ needs to be replaced by $S_{\partial G}$ which then leads to the matrix which contains the field reconstructions

$$
\boldsymbol{u}_{\alpha}^{s}:=S_{\partial G} \circ \boldsymbol{R}_{\alpha} \circ \boldsymbol{u}^{\infty}
$$

with $n_{l}$ points $z_{1}, \ldots, z_{n_{l}}$ (rows) for each $d \in \boldsymbol{\Lambda}$ (columns). Finally, the scattered fields for all positive test domains are combined as described in (4.11).

Examples of the modified Kirsch-Kress method can be found in Figure 5.3 and Figure 5.4 for Dirichlet and Neumann case respectively. In both cases the test domains are circle with radius of 4 and are evaluated with 100 points on the boundary. The sampling grid has $100 \times 100$ points.

\subsection{Multiwave range test}

Here, the numerical realization of the multiwave range test according to Algorithm 3 is described. Using $\boldsymbol{u}_{\alpha}^{\mathcal{S}}(x)$ given in (5.4), the numerical evaluation of the density $\psi_{x}$ is carried out by

$$
\begin{aligned}
\boldsymbol{\psi}_{x} & =\gamma_{m} \boldsymbol{R}_{\alpha} \circ\left(\boldsymbol{u}_{\alpha}^{s}(x)\right)^{T} \\
& =\gamma_{m} \boldsymbol{R}_{\alpha} \circ\left(\boldsymbol{S}_{\partial G, x} \circ \boldsymbol{R}_{\alpha} \circ \boldsymbol{u}^{\infty}\right)^{T}
\end{aligned}
$$




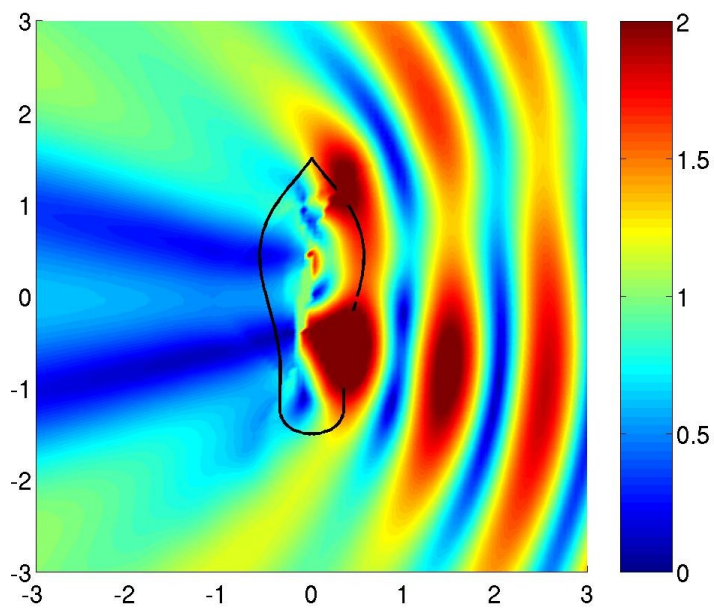

(a) Reconstructed total field

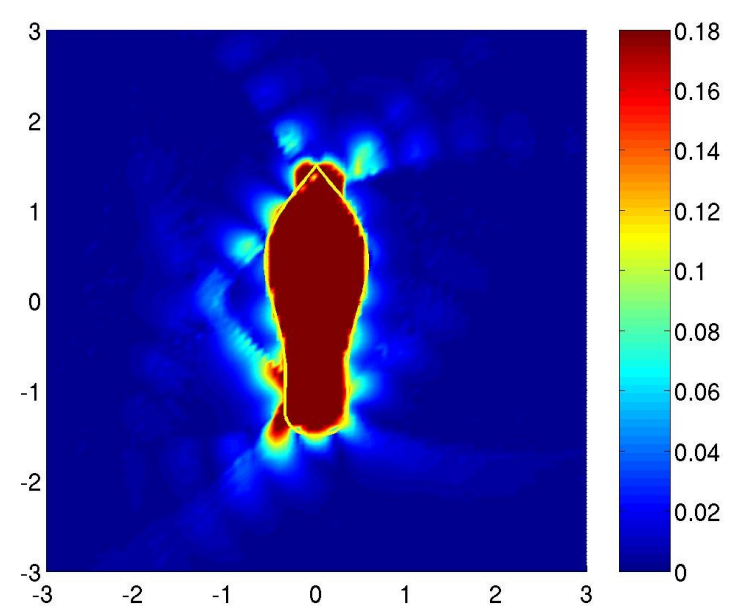

(b) Difference to the true solution

Figure 5.4: Field reconstructions with the modified method of Kirsch-Kress in the case of Neumann boundary conditions for a boat-like scatterer with an incident plane wave coming from the right with $\kappa=3$. The test domains have a radius of 4 .

Note that using the same $\boldsymbol{R}_{\alpha}$ twice is only possible if the number of incoming waves is equal to the number of farfield discretisation points. When they are different, also two different matrix operators are necessary. The norm evaluation is used as

$$
\mu(x)=\sqrt{\psi_{x}^{T} \cdot \psi_{x}} .
$$

Finally, proceed as in equations (4.26) to build up a boolean function $b(j, x)$ for $x$ in a sampling grid $\mathcal{M}$ and then use the minimum over all $b(j, x)$ (cf. 4.27) to find a reconstruction to the unknown shape of the scatterer.

In Figure 5.5 reconstructions of an boat-like scatterer are shown. 100 incident plane waves are used with wave number $\kappa=3$. The test domains have 100 evaluation points on the boundary and the number of configurations $\Theta$ were 20.

\subsection{Singular sources method - alternative}

Finally, the numerical realization of the new approach to the singular sources method is described. The reconstruction of $\Phi^{S}(z, z)$ is based on equation (5.6). An application of the potential evaluation can be used to calculate

$$
\boldsymbol{\Phi}_{\alpha}^{S}(z, z):=\gamma_{m} \boldsymbol{R}_{\alpha} \circ\left(\boldsymbol{S}_{\partial G, x} \circ \boldsymbol{R}_{\alpha} \circ \boldsymbol{u}^{\infty}\right)^{T}
$$

as an approximation to the scattered field $\Phi^{S}(z, z)$ for an incident point source with source point $z$. The value of $\left|\Phi^{S}(z, z)\right|$ can be used according to (4.28) to detect the shape of $\partial D$.

Reconstructions $\Phi_{\alpha}^{s}(z, z)$ of an boat-like obstacle with the method are shown in Figure 5.6. The parameters were chosen as $\kappa=3$ and there were 100 incident plane waves and measurement points in $\mathcal{M}$. 


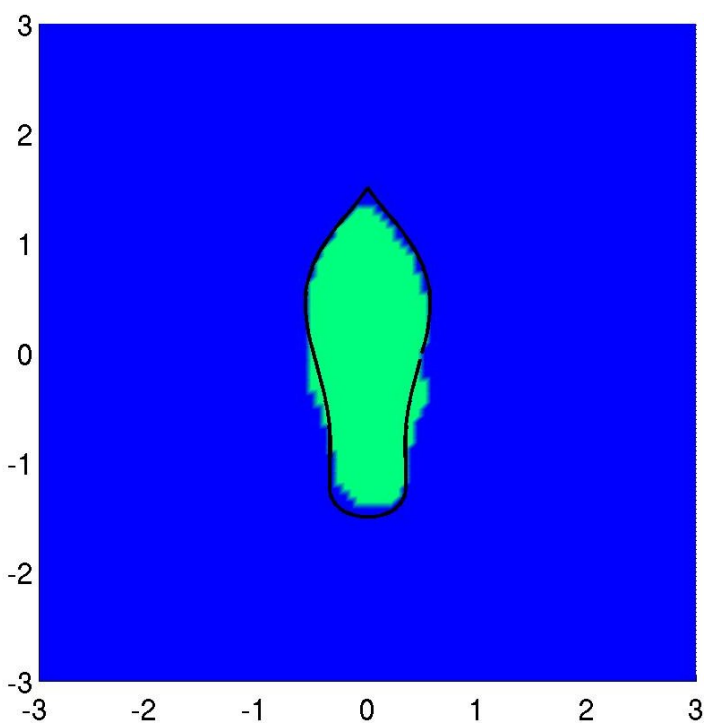

(a) Dirichlet boundary condition

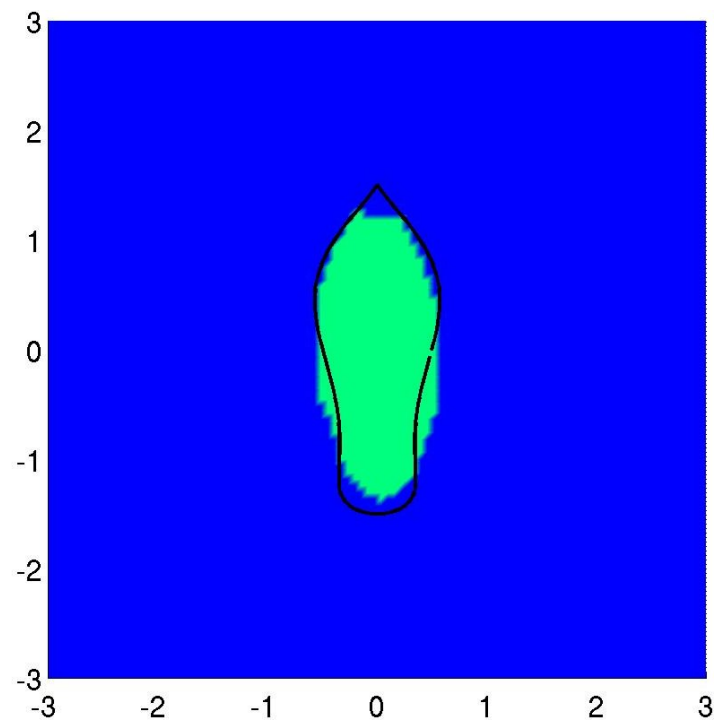

(b) Neumann boundary condition

Figure 5.5: Reconstruction of the shape with the multiwave range test with 100 incident plane waves with $\kappa=3$, 100 evaluation points and 20 configurations.

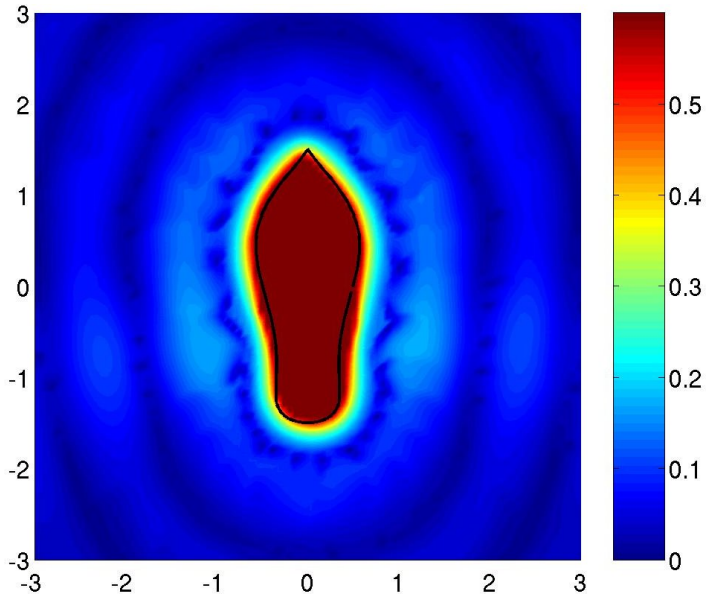

(a) Dirichlet boundary conditions

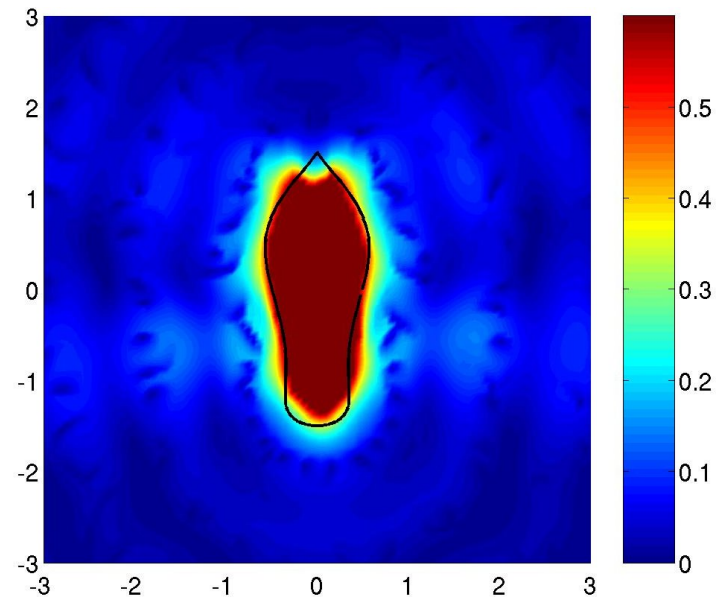

(b) Neumann boundary conditions

Figure 5.6: Reconstruction of the shape with the singular sources method for a boat shaped domain. Here $\kappa=3$ and 100 incident waves and measurement points are used. 
Part II

Electromagnetic scattering 

In this part the focus lies on electromagnetic scattering in a two-layered media with application to mine detection, especially with handheld detectors.

The part is, like the acoustic part, organised in four chapters. The first chapter covers the setting and tools (Chapter 6), the second chapter focuses on the direct problem (Chapter 7), the third is about the inverse problem (Chapter 8) and finally the fourth describes the numerical implementation (Chapter 9) and gives examples of the direct and inverse problem. 


\section{Chapter 6}

\section{Setting and tools}

In this chapter the setting and tools for the electromagnetic scattering problem under consideration are described. In Section 6.1 the pieces for the scattering problem are collected. Further, the necessary tools for the theory for the forward and the inverse problem are summed up in Section 6.2.

\subsection{Definition}

First, the physical setting and its notation for the domains under consideration is introduced.

Definition 6.1.1 (Domains). Assume $D_{3} \subset \mathcal{D}_{2}$ to be a bounded obstacle of class $C^{2}$ with connected complement. In the following, for simplicity, $D$ is written for $D_{3}$ most of the time. Further, let $v$ be the outward unit normal to $D$ and the upward unit normal to $S$.

The free space without the obstacle is denoted as $D_{0}:=\mathbb{R}^{3} \backslash \bar{D}$. Let $\mathcal{D}_{1}=D_{1}:=$ $\left\{x=\left(x_{1}, x_{2}, x_{3}\right) \in \mathbb{R}^{3}: x_{3}>0\right\}$ be the upper half-space and $\mathcal{D}_{2}:=\left\{x \in \mathbb{R}^{3}: x_{3}<0\right\}$ the lower half-space. The lower halfspace without the obstacle is written as $D_{2}:=$ $\mathcal{D}_{2} \backslash \bar{D}$. Furthermore $S_{1}:=\left\{x \in \mathbb{R}^{3}: x_{3}=0\right\}$ denotes the interface between $\mathcal{D}_{1}$ and $\mathcal{D}_{2}$. Summarising, the case $j=0$ is used for the free space, $j=3$ denotes the domain of a penetrable obstacle and $j=1,2$ are used for the two half-spaces.

In the framework of mine detection this setting is illustrated in Figure 6.1. Consider the propagation of electromagnetic waves with frequency $\omega$ in a two-layered medium consisting of the two isotropic half-spaces $D_{j}$ with electric permittivity $\epsilon_{j}$, magnetic permeability $\mu_{j}$ and electric conductivity $\sigma_{j}$ for $j=0,1,2,3$. The SI units of these parameters are $[\omega]=1 / \mathrm{s},[\epsilon]=\mathrm{As} / \mathrm{Vm},[\mu]=\mathrm{Vs} / \mathrm{Am}$ and $[\sigma]=\mathrm{A} / \mathrm{Vm}$.

\subsubsection{Time-dependent and time-harmonic Maxwell equations}

An electromagnetic wave is described by the electric field $\mathcal{E}_{j}=[\mathrm{V} / \mathrm{m}]$ and the magnetic field $\mathcal{H}_{j}=[\mathrm{A} / \mathrm{m}]$. These quantities satisfy the Maxwell equations

$$
\operatorname{curl} \mathcal{E}_{j}+\mu_{j} \frac{\partial \mathcal{H}_{j}}{\partial t}=0, \quad \operatorname{curl} \mathcal{H}_{j}-\epsilon_{j} \frac{\partial \mathcal{E}_{j}}{\partial t}=\sigma_{j} \mathcal{E}_{j}
$$




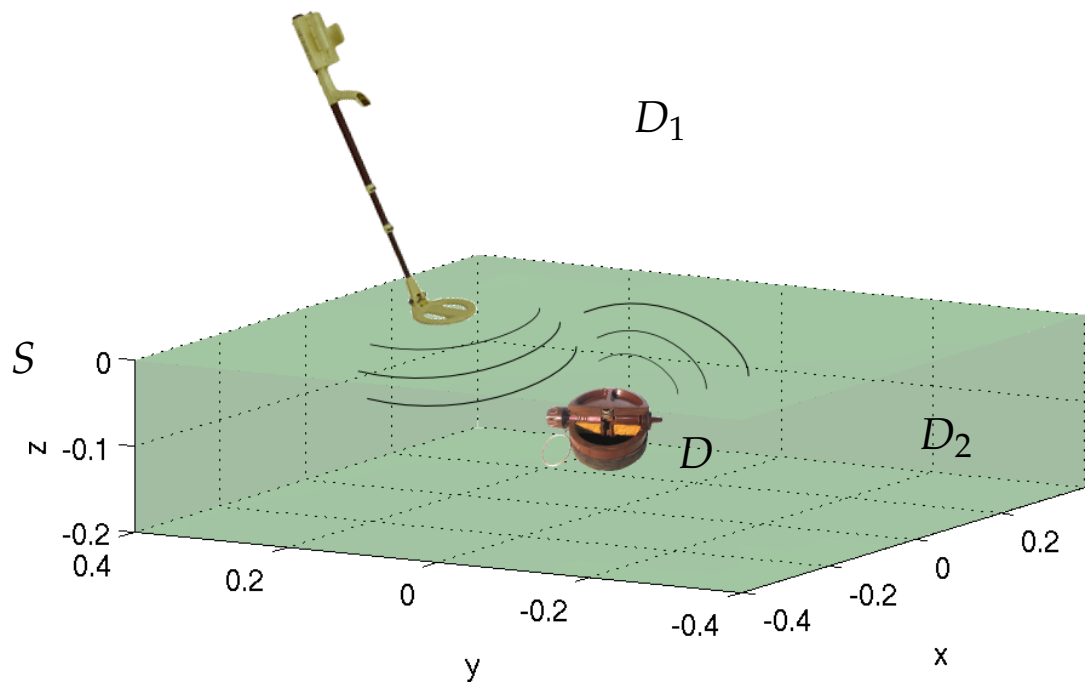

Figure 6.1: Common setting for mine detection with a handheld detector.

The electric and magnetic fields can be viewed as a superposition of time-harmonic fields. This approach is applicable if the scattering object does not move in time. Time-harmonic fields of frequency $\omega$ have the form

$$
\begin{aligned}
& \mathcal{E}_{j, \omega}(x, t)=\Re\left\{\left(\epsilon_{j}+\frac{i \sigma_{j}}{\omega}\right)^{-1 / 2} E_{j}(x) e^{-i \omega t}\right\}, \\
& \mathcal{H}_{j, \omega}(x, t)=\Re\left\{\mu_{j}^{-1 / 2} H_{j}(x) e^{-i \omega t}\right\} .
\end{aligned}
$$

A general pulse can be modelled by the Fourier transform and yields

$$
\begin{aligned}
& \mathcal{E}_{j}(x, t)=\Re\left\{\int_{-\infty}^{\infty}\left(\epsilon_{j}+\frac{i \sigma_{j}}{\omega}\right)^{-1 / 2} E_{j, \omega}(x) e^{-i \omega t} \mathrm{~d} \omega\right\}, \\
& \mathcal{H}_{j}(x, t)=\Re\left\{\int_{-\infty}^{\infty} \mu_{j}^{-1 / 2} H_{j, \omega}(x) e^{-i \omega t} \mathrm{~d} \omega\right\},
\end{aligned}
$$

where it is assumed that $E_{j, \omega}$ and $H_{j, \omega}$ are square integrable and sufficiently smooth with respect to $\omega$, i.e. the change in time is small. In these cases the Maxwell equations (6.1) are reduced to the symmetric time-harmonic Maxwell equations

$$
\begin{aligned}
& \operatorname{curl} E_{j}-i \kappa H_{j}=0 \\
& \operatorname{curl} H_{j}+i \kappa E_{j}=0
\end{aligned}
$$

for the field $E_{j}=E_{j, \omega}$ and $H_{j}=H_{j, \omega}$, where the wave number $([\kappa]=1 / \mathrm{m})$ is defined as

$$
\kappa_{j}=\sqrt{\left(\epsilon_{j}+\frac{i \sigma_{j}}{\omega}\right) \mu_{j} \omega^{2}}
$$

with the complex square root chosen such that $\Im \kappa_{j} \geq 0$. The wave number is assumed to be a complex number in every domain with the exception of $D_{1}$, where it can be real or complex. 
This time-harmonic representation is used throughout this work, which by the Fourier transform covers a full time-dependent scattering process.

The electric field $E_{j}$ and the electric flow density $D_{j}$ as well as the magnetic field $H_{j}$ and the magnetic flow $B_{j}$ are connected by the material equations

$$
D_{j}=\epsilon_{j} E_{j}, \quad B_{j}=\mu_{j} H_{j},
$$

but which are not further used in the scope of this work.

The following Theorem connects the cartesian components of the time harmonic Maxwell equations to the Helmholtz equation, which is one step to be able to use the results for the Helmholtz equation also in the framework of time-harmonic electromagnetics. The other step will be to show the componentwise equivalence of the radiation conditions.

Theorem 6.1.2 (Vector Helmholtz equation). Let the fields $E_{j}, H_{j}$ be solutions to the time harmonic Maxwell equations (6.4). Then $E$ and $H$ are divergence free and satisfy the vector Helmholtz equation

$$
\Delta E_{j}+\kappa_{j}^{2} E_{j}=0 \text { and } \Delta H_{j}+\kappa_{j}^{2} H_{j}=0 .
$$

Conversely, let the divergence free field $E_{j}$ or $H_{j}$ be a solution to the vector Helmholtz equation. Then $E_{j}$ and $H_{j}:=\operatorname{curl} E_{j} / i \kappa$ or $H_{j}$ and $E_{j}:=-\operatorname{curl} H_{j} / i \kappa$ satisfy the Maxwell equations (6.4).

Proof. Using the vector identity

$$
\operatorname{curl} \operatorname{curl} E_{j}=-\Delta E_{j}+\operatorname{grad} \operatorname{div} E_{j}
$$

and the time harmonic Maxwell equations in

$$
\Delta E_{j}+\kappa^{2} E_{j}
$$

leads to

$$
\Delta E_{j}+\kappa^{2} E_{j}=-i \kappa \operatorname{curl} H_{j}-\frac{1}{i \kappa} \operatorname{grad} \operatorname{div} \operatorname{curl} H_{j}+\kappa^{2} E_{j}
$$

The second term trivially is zero. Again using the Maxwell equations to replace the first term gives

$$
\Delta E_{j}+\kappa^{2} E_{j}=-\kappa^{2} E_{j}+\kappa^{2} E_{j}=0 .
$$

For the other direction use (6.7) on the first equation of (6.6). Then with $H_{j}:=$ $\operatorname{curl} E_{j} / i \kappa(6.7)$ becomes

$$
\operatorname{curl} H+i \kappa E=0
$$

This proves the vector Helmholtz equation for $E_{j}$. For $H_{j}$ the proof is analogously. 


\subsubsection{Transmission conditions}

For the layered case the fields require the transmission conditions at the air-soil interface $S_{1}$ to be

$$
v \times E_{1}=a_{E} v \times E_{2}, \quad v \times H_{1}=a_{H} v \times H_{2} \quad \text { on } S_{1},
$$

with constants $a_{E}, a_{H} \in \mathbb{C} \backslash\{0\}$. For the applications in mine detection $D_{1}$ is air which is non-conducting, i.e. $\sigma_{1}=0$ and consequently $\Im \kappa_{1}=0$. However, in $D_{2}$ there is soil with a conductivity of $\sigma_{2}>0$ and therefore $\Im \kappa_{2}>0$. The constants $a_{E}$ and $a_{H}$ are then given by

$$
a_{E}=\sqrt{\frac{\epsilon_{1}}{\epsilon_{2}+\frac{i \sigma_{2}}{\omega}}}, \quad a_{H}=\sqrt{\frac{\mu_{1}}{\mu_{2}}} .
$$

These transmission conditions are a consequence of the symmetric form (6.2) of the harmonic time dependence and the physical requirements, which is that the continuity of the tangential components of the electric field $\mathcal{E}$ and the magnetic field $\mathcal{H}$ across the interface is required.

\subsubsection{Boundary condition}

Incident and scattered electric or magnetic fields, respectively, are interconnected by boundary conditions. For perfectly conducting scatterers the tangential component $v \times E$ of the total electric field $E$ has to vanish on the boundary, i.e. the metallic object $D$ is described by the perfect conductor boundary condition

$$
v \times E=0 \text { on } \partial D,
$$

which is a reasonable approximation for highly-conducting metals. Instead of the electric field $E$ the boundary conditions always can be written in terms of $H$ through the use of the Maxwell equations (6.4). The more general case of non-perfectly conducting obstacles is modeled via a transmission boundary condition

$$
\begin{aligned}
v(x) \times E_{j}(x)-b_{E} v(x) \times E_{3}(x) & =0 \\
v(x) \times H_{j}(x)-b_{H} v(x) \times H_{3}(x) & =0
\end{aligned}
$$

for $j=0,2$ with constants $b_{E}$ and $b_{H}$ on the boundary $\partial D$ of the obstacle. Due to the continuity of the tangential components across the boundary the constants are given by

$$
b_{E}=\sqrt{\frac{\epsilon_{0}+\frac{i \sigma_{0}}{\omega}}{\epsilon_{3}+\frac{i \sigma_{3}}{\omega}}}, \quad b_{H}=\sqrt{\frac{\mu_{1}}{\mu_{3}}} .
$$

for the full space transmission problem and

$$
b_{E}=\sqrt{\frac{\epsilon_{2}+\frac{i \sigma_{2}}{\omega}}{\epsilon_{3}+\frac{i \sigma_{3}}{\omega}}}, \quad b_{H}=\sqrt{\frac{\mu_{2}}{\mu_{3}}} .
$$

for the layered space transmission problem. 


\subsubsection{Radiation condition and Rellich}

To characterise physically relevant solutions, in view of the real wave number $\kappa_{1}$ the scattered field $E_{1}^{s}, H_{1}^{s}$ is required to satisfy the Silver-Müller radiation condition

$$
\lim _{|x| \rightarrow \infty}\left(H_{1}^{S} \times x-|x| E_{1}^{S}\right)=0
$$

uniformly for all directions $\hat{x}=x\|x\|$ in the upper half-space $D_{1}$ or in $\mathbb{R}^{3} \backslash \bar{D}$ for the full space case. An alternative form of the radiation condition is

$$
\frac{x}{|x|} \times H_{1}(x)+E_{1}(x)=o\left(\frac{1}{|x|}\right), \quad x \rightarrow \infty
$$

and

$$
E_{1}(x)=O\left(\frac{1}{|x|}\right), \quad x \rightarrow \infty
$$

uniformly for all directions in the upper half-space $D_{1}$ or in $\mathbb{R}^{3} \backslash \bar{D}$. For $x \in D_{2}$ and the wave number $\kappa_{2}$ with $\Im \kappa_{2}>0$ explicitly assume an exponential decay

$$
\left|E_{2}^{S}(x)\right|+\left|H_{2}^{S}(x)\right| \leq M \exp \left(-\Im \kappa_{2}|x|\right)
$$

with some constant $M>0$.

Solutions to the Maxwell equations which fulfil this radiation condition are called radiating solutions.

Theorem 6.1.3 (Equivalence of radiation conditions). The Silver-Müller radiation condition is equivalent to the Sommerfeld radiation condition for the cartesian components of solutions to the Maxwell equations.

Proof. Express the solutions to the Maxwell equations as magnetic and electric dipole distributions, as implied through the Stratton-Chu formulas (Theorem B.2.1). With straightforward calculations it can be shown, that the cartesian components of the magnetic and electric dipole satisfies the Sommerfeld radiation condition uniformly for all $y \in \partial D$. Then, from the Stratton Chu formulas, the cartesian components of solutions to the Maxwell equations satisfy the Sommerfeld radiation condition.

For the other direction, start with looking at the asymptotic

$$
\operatorname{curl} a \Phi(x, y) \times x+x \operatorname{div} a \Phi(x, y)-i k|x| a \Phi(x, y)=O\left(\frac{|a|}{|x|}\right), \quad x \rightarrow \infty
$$

uniformly for all directions $\frac{x}{|x|}$ and all $y \in \partial D$. The same asymptotic holds true for $\partial \Phi(x, y) / \partial v(y)$ instead of $\Phi(x, y)$. Then with Green's formula (Theorem B.1.2) and the vector Helmholtz equation (Theorem 6.1.2) the solutions to the Maxwell equations which satisfy the Sommerfeld radiation condition componentwise also satisfy the Silver-Müller radiation condition. This completes the proof.

Remark. Together with the vector Helmholtz equation (Theorem 6.1.2) this opens up the possibility of using results for the Helmholtz equation also in the electromagnetic framework under consideration. 
With the condition of radiating solutions Rellich's Lemma (Lemma 2.1.4) and its extension (Lemma 3.1.2) can be transfered from the acoustic case to the electromagnetic case.

Lemma 6.1.4 (Extended Rellich). Assume that the bounded set $D$ is the open complement of an unbounded domain and let $E, H \in C^{1}\left(\mathbb{R}^{3} \backslash \bar{D}\right) \cap C\left(\mathbb{R}^{3} \backslash D\right)$ be a radiating solution to the time harmonic Maxwell equations satisfying

$$
\Re \int_{\partial D} v \times E \cdot \bar{H} \mathrm{~d} s \leq 0 .
$$

Then $E=H=0$ in $\mathbb{R}^{3} \backslash \bar{D}$.

Proof. Let $B_{R}:=\left\{x \in \mathbb{R}^{3}:|x|<R\right\}$ with radius $R>0$ be a ball. Then the SilverMüller radiation condition (6.11) implies

$$
\begin{aligned}
& \lim _{R \rightarrow \infty} \int_{\partial B_{R}}\left\{|H \times v|^{2}+|E|^{2}-2 \Re(v \times E \cdot \bar{H})\right\} \mathrm{d} s \\
& =\lim _{R \rightarrow \infty} \int_{\partial B_{R}}|H \times v-E|^{2} \mathrm{~d} s=0 .
\end{aligned}
$$

Using the Gauss divergence theorem in the domain $B_{R} \backslash D$ leads to

$$
\int_{B_{R} \backslash D} \operatorname{div}(E \times \bar{H}) \mathrm{d} x=\int_{\partial B_{R}} v \times E \cdot \bar{H} \mathrm{~d} s-\int_{\partial D} v \times E \cdot \bar{H} \mathrm{~d} s .
$$

Then, with the vector-identity

$$
\operatorname{div}(E \times \bar{H})=\operatorname{curl} E \cdot \bar{H}-E \cdot \operatorname{curl} \bar{H}
$$

and the Maxwell equations, (6.16) is transformed to

$$
\begin{aligned}
\int_{\partial B_{R}} v \times E \cdot \bar{H} \mathrm{~d} s= & i \int_{B_{R} \backslash D}\left\{\kappa|H|^{2}-\bar{\kappa}|E|^{2}\right\} \mathrm{d} x \\
& +\int_{\partial D} v \times E \cdot \bar{H} \mathrm{~d} s
\end{aligned}
$$

Then, inserting the real part of (6.18) into (6.15) leads to

$$
\lim _{R \rightarrow \infty} \int_{\partial B_{R}}\left\{|H \times v|^{2}+|E|^{2}\right\} \mathrm{d} s=2 \Re \int_{\partial D} v \times E \cdot \bar{H} \mathrm{~d} s .
$$

With the assumption of the Theorem all terms on the left hand side of (6.19) individually have to tend to zero. Then, from the vector Helmholtz equations (Theorem 6.1.2) and the equivalence of the radiation conditions (Theorem 6.1.3) the assumption of Rellichs lemma (2.1.4) for the Helmholtz equation is satisfied for the cartesian components of the fields $E, H$. Then apply Rellichs lemma to complete the proof.

\subsection{Solution tools}

In this section the fundamental solution and Green's tensors for the setting at hand are developed and analysed. Further, following Huygens principle, the surface potentials are introduced, which form the basis of the solution theory. 

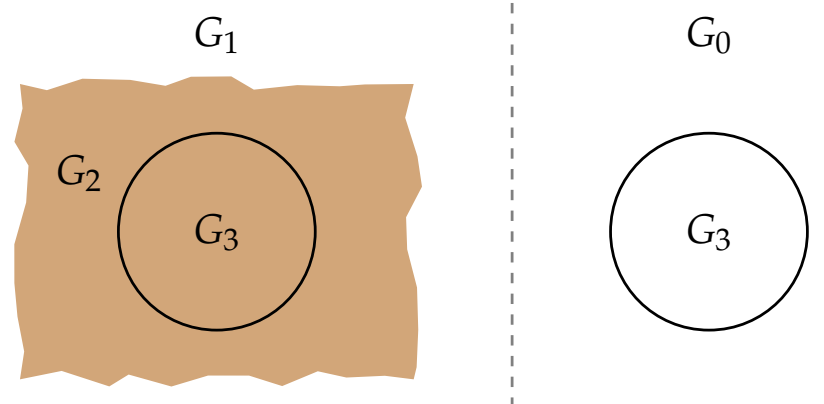

Figure 6.2: Scheme where the different Green's tensors are valid. Left: layered case. Right: free space.

\subsubsection{Fundamental solution and Green's tensor}

A central tool for the description and solution of electromagnetic scattering is the fundamental solution of the Helmholtz equation and the Green's tensor. In this work the notation $G_{E, j}, G_{H, j}, j=0,1,2,3$ is used for the Green's tensor for obstacle scattering in all background cases whereas the case $j=0$ describes the free space $(x \in \mathbb{R} \backslash \bar{D})$, $j=1$ denotes the upper halfspace (e.g. air) $\left(x \in D_{1}\right), j=2$ denotes the lower halfspace (e.g. clay) of the layered medium $\left(x \in D_{2}\right)$ and $j=3$ is used for a penetrable object $\left(x \in D_{3}\right)$. The areas for which the Green's tensors are used is also shown in Figure 6.2.

The Green's tensor for both the free space and the layered medium has to be constructed in such a way that it gives the solution for a point source (or dirac function). Here, the fundamental solution to the Helmholtz equation (compare Definition 2.2.1) in $D_{j}, j=0,1,2,3$ is denoted by

$$
\Phi_{j}(x, y)=\frac{1}{4 \pi} \frac{e^{i \kappa_{j}|x-y|}}{|x-y|}, x \neq y .
$$

When used without index $j$ the wave number $\kappa$ is not fixed to a specific value.

Definition 6.2.1 (Green's tensor, free space). For all $x, y \in D_{j}$ with $j=0,3$ let the Green's tensor for the free space defined by

$$
\begin{aligned}
& G_{E, j}(x, y) p:=\operatorname{curl}_{x} \Phi_{j}(x, y) p \\
& G_{H, j}(x, y) p:=\frac{1}{i \kappa_{j}} \operatorname{curl}_{x} \operatorname{curl}_{x} \Phi_{j}(x, y) p,
\end{aligned}
$$

for all polarisations $p \in \mathbb{R}^{3}$ and $j=0,3$, which corresponds to an magnetic dipole.

Theorem 6.2.2 (properties of free space Green's tensor). The pairs of corresponding columns of the Green's tensor for free space $G_{E, j}$ and $G_{H, j}$ for $j=0,3$ satisfy the Maxwell equations (6.4) in $\mathbb{R}^{3} \backslash\{y\}$ and the Silver-Müller radiation conditions (6.11). 
Proof. The Maxwell equations are satisfied directly from the definition of the tensor and the vector Helmholtz equation (Theorem 6.1.2). The equivalence of the radition conditions then proof that the Green's tensor satisfy the Silver-Müller radiation condition.

Definition 6.2.3 (Green's tensor, layered media). For all $x \in D_{j}$ with $j=1,2$ and fixed $y \in D_{L}$ with $L=1,2$ let the Green's tensor for a two layered medium be given as

$$
\begin{aligned}
G_{E, j}(x, y) p & := \begin{cases}\operatorname{curl}_{x} \Phi_{j}(x, y) p+\widetilde{G}_{E, j}(x, y) p & , \text { for } j=L \\
\widetilde{G}_{E, j}(x, y) p & , \text { for } j \neq L\end{cases} \\
G_{H, j}(x, y) p & :=\frac{1}{i k_{j}} \operatorname{curl}_{x} G_{E, j}(x, y) p,
\end{aligned}
$$

for all polarisations $p \in \mathbb{R}^{3}$ and the smooth matrix $\widetilde{G}_{E, j}$, which is derived in Section 6.2.2. The $G_{E, j}, G_{H, j}$ have a singularity of the form of the electromagnetic field of a magnetic dipole located in the source point $y \in D_{L}, L=1,2$.

Theorem 6.2.4 (properties of layered space Green's tensor). The pairs of corresponding columns of the Green's tensor for layered space $G_{E, j}$ and $G_{H, j}$ with $j=1,2$ satisfy the Maxwell equations (6.4) in $\mathbb{R}^{3} \backslash\{y\}$, the Silver-Müller radiation conditions (6.11), the decay condition (6.14) and the transmission conditions (6.8).

Remark. The Maxwell equations are satisfied directly from the definition of the tensor and the vector Helmholtz equation (Theorem 6.1.2). The equivalence of the radition conditions then proof that the Green's tensor satisfy the Silver-Müller radiation condition. The decay condition and the transmission condition is fulfilled via the construction of the Green's tensor derived in Section 6.2.2.

Remark. Since (6.21) has the same singularity as (6.20) and only differs with a smooth matrix, it follows that all the regularity results for the surface potentials and boundary integral operators (see Definition 6.2.11, Definition 6.2.12 and [8]) can be proven analogously for the layered medium case.

\subsubsection{Derivation of the Green's tensor}

For the construction of the Green's tensor a simplified version of the approach in [33] is used here. The derivation is written up in the submitted paper [10]. In [33] the Green's tensor is constructed for an arbitrary number of layers and uses a slightly different ansatz which turns out to be more complicated in the end. The approach described here is also more systematic than the presentation in [28] that is based on Hertz vectors and that provides only the tensor for the magnetic fields.

Recall the definition of the Green's tensor (Definition 6.2.3). The main idea for its construction is to reduce the transmission problem for the Maxwell equations to a 
transmission problem for the Helmholtz equation. To this end denote the canonical unit vectors in $\mathbb{R}^{3}$ by $e_{1}, e_{2}$ and $e_{3}$ and begin with the following lemma.

Lemma 6.2.5. For $j=1,2$, let $u_{j}$ and $v_{j}$ satisfy the Helmholtz equations with wave numbers $\kappa_{j}$ in $\mathcal{D}_{j}$ and the transmission conditions

$$
\kappa_{1} u_{1}=a_{H} \kappa_{2} u_{2}, \quad \frac{\partial u_{1}}{\partial v}=a_{E} \frac{\partial u_{2}}{\partial v} \quad \text { on } S_{1}
$$

and

$$
v_{1}=a_{E} v_{2}, \quad \frac{1}{\kappa_{1}} \frac{\partial v_{1}}{\partial v}=\frac{a_{H}}{\kappa_{2}} \frac{\partial v_{2}}{\partial v} \text { on } S_{1}
$$

Then the fields

$$
E_{j}:=\operatorname{curl} \operatorname{curl}\left\{u_{j} e_{3}\right\}+\operatorname{curl}\left\{v_{j} e_{3}\right\}, \quad H_{j}:=\frac{1}{i k_{j}} \operatorname{curl} E_{j}
$$

satisfy the Maxwell equations (6.4) with wave numbers $\kappa_{j}$ in $\mathcal{D}_{j}$ and the transmission conditions (6.8)

$$
v \times E_{1}=a_{E} v \times E_{2}, \quad v \times H_{1}=a_{H} v \times H_{2} \quad \text { on } S_{1} .
$$

Proof. Note for the proof that $v$ on $S_{1}$ is identical to $e_{3}$. From

$$
\operatorname{curl} \operatorname{curl}\left\{u_{j} e_{3}\right\}=\kappa_{j}^{2} u_{j} e_{3}+\operatorname{grad}\left\{\operatorname{grad} u_{j} \cdot e_{3}\right\}
$$

conclude that

$$
v \times \operatorname{curl} \operatorname{curl}\left\{u_{j} e_{3}\right\}=v \times \operatorname{Grad} \frac{\partial u_{j}}{\partial v} \quad \text { on } S_{1} .
$$

Furthermore, using the vector identity $\operatorname{curl}(U v)=U \operatorname{curl} v+(\operatorname{grad} U) \times v$ the following holds

$$
v \times \operatorname{curl}\left\{v_{j} e_{3}\right\}=v \times\left\{\operatorname{grad} v_{j} \times v\right\} \quad \text { on } S_{1} .
$$

Now the last two identities and

$$
H_{j}=-i \kappa_{j} \operatorname{curl}\left\{u_{j} e_{3}\right\}+\frac{1}{i k_{j}} \operatorname{curl} \operatorname{curl}\left\{v_{j} e_{3}\right\}
$$

immediately ensure that (6.22) and (6.23) imply that the transmission conditions (6.25) are satisfied.

Now introduce notations and basic functions along with their properties for the remainder of this derivation for the Green's tensor. For $x=\left(x_{1}, x_{2}, x_{3}\right)$ and $y=$ $\left(y_{1}, y_{2}, y_{3}\right)$ in $\mathbb{R}^{3}$ denote

$$
r:=\sqrt{\left(x_{1}-y_{1}\right)^{2}+\left(x_{2}-y_{2}\right)^{2}}
$$

and for $\lambda \in \mathbb{R}$ set

$$
\alpha:=\sqrt{\lambda^{2}-\kappa^{2}}
$$


with $\Re \alpha>0$. Further abbreviate $e_{\ell}^{\perp}=e_{3} \times e_{\ell}, \ell=1,2$, that is, $e_{1}^{\perp}=e_{2}$ and $e_{2}^{\perp}=-e_{1}$, also $\{x-y\}_{\mathrm{t}}=\left(x_{1}-y_{1}, x_{2}-y_{2}, 0\right)$ and introduce

$$
\begin{aligned}
& \Psi_{\ell}(x, y):=\operatorname{sgn}\left(x_{3}-y_{3}\right) e^{-\alpha\left|x_{3}-y_{3}\right|} e_{\ell} \cdot\{x-y\} \frac{J_{1}(\lambda r)}{r}, \\
& \chi_{\ell}(x, y):=\frac{1}{\alpha} e^{-\alpha\left|x_{3}-y_{3}\right|} e_{\ell}^{\perp} \cdot\{x-y\} \frac{J_{1}(\lambda r)}{r}, \\
& \Theta(x, y):=\frac{\lambda}{\alpha} e^{-\alpha\left|x_{3}-y_{3}\right|} J_{0}(\lambda r),
\end{aligned}
$$

where $J_{0}$ and $J_{1}$ denote the Bessel function (B.12) of order zero and one, respectively. Following the proof of the Sommerfeld identity (Theorem B.3.6) the function $\Theta$ solve the Helmholtz equation with wave number $\kappa$. From (B.11) $f(\kappa r)$ can be identified with $J_{1}(\lambda r)$ and $e_{\ell}^{\perp} \cdot\{x-y\} / r$ and $e_{\ell} \cdot\{x-y\} / r$ form the two dimensional spherical harmonics of order 1 , therefore the functions $\Psi_{\ell}$ and $\chi_{\ell}$ solve the Helmholtz equation in $2 \mathrm{D}$ again from following the proof of the Sommerfeld identity (Theorem B.3.6).

For the following straightforward calculations the vector identity

$$
\operatorname{curl}(U v)=U \operatorname{curl} v+\operatorname{grad} U \times v
$$

with scalar $U$ and vector $v$ is used. Further, using the product rule, the Bessel differential equation (Theorem B.3.1) for $J_{0}$ and the recurance relation $J_{0}^{\prime}=-J_{1}$ (from (B.15) and (B.16)) leads to

$$
\frac{\mathrm{d}}{\mathrm{d} r} \frac{J_{1}(\lambda r)}{r}=\frac{\lambda J_{0}(\lambda r)}{r}-2 \frac{J_{1}(\lambda r)}{r^{2}} .
$$

Then, the following holds true

$$
\begin{aligned}
\operatorname{grad}\left(\frac{J_{1}(\lambda r}{r}\right) & =\frac{(x-y)}{r} \frac{\mathrm{d}}{\mathrm{d} r} \frac{J_{1}(\lambda r)}{r} \\
& =\frac{(x-y)}{r^{2}}\left(\lambda J_{0}(\lambda r)-2 \frac{J_{1}(\lambda r)}{r}\right) .
\end{aligned}
$$

Now, using the three above equations for calculating the curls of (6.28) leads to

$$
\begin{aligned}
\operatorname{curl}\left\{\Psi_{\ell} e_{3}\right\}(x, y)= & \operatorname{sgn}\left(x_{3}-y_{3}\right) e^{-\alpha\left|x_{3}-y_{3}\right|}\left(-\frac{J_{1}(\lambda r)}{r} e_{\ell}^{\perp}\right. \\
& \left.+e_{\ell} \cdot\{x-y\}\left(\frac{\lambda J_{0}(\lambda r)}{r^{2}}-2 \frac{J_{1}(\lambda r)}{r^{3}}\right)\{x-y\} \times e_{3}\right) .
\end{aligned}
$$

and

$$
\begin{aligned}
\operatorname{curl} \operatorname{curl}\left\{\Psi_{\ell} e_{3}\right\}(x, y)= & e^{-\alpha\left|x_{3}-y_{3}\right|}\left(-\alpha \frac{J_{1}(\lambda r)}{r} e_{\ell}\right. \\
& +\lambda^{2} \operatorname{sgn}\left(x_{3}-y_{3}\right) e_{\ell} \cdot\{x-y\} \frac{J_{1}(\lambda r)}{r} e_{3} \\
& \left.-\alpha e_{\ell} \cdot\{x-y\}\left(\frac{\lambda J_{0}(\lambda r)}{r^{2}}-2 \frac{J_{1}(\lambda r)}{r^{3}}\right)\{x-y\}_{\mathrm{t}}\right),
\end{aligned}
$$


as well as

$$
\begin{aligned}
\operatorname{curl}\left\{\chi_{\ell} e_{3}\right\}(x, y)= & \frac{e^{-\alpha\left|x_{3}-y_{3}\right|}}{\alpha}\left(\frac{J_{1}(\lambda r)}{r} e_{\ell}\right. \\
& \left.+e_{\ell}^{\perp} \cdot\{x-y\}\left(\frac{\lambda J_{0}(\lambda r)}{r^{2}}-2 \frac{J_{1}(\lambda r)}{r^{3}}\right)\{x-y\} \times e_{3}\right)
\end{aligned}
$$

and

$$
\begin{aligned}
\operatorname{curlcurl}\left\{\chi_{\ell} e_{3}\right\}(x, y)= & \operatorname{sgn}\left(x_{3}-y_{3}\right) e^{-\alpha\left|x_{3}-y_{3}\right|}\left(-\frac{J_{1}(\lambda r)}{r} e_{\ell}^{\perp}\right. \\
& +\frac{\lambda^{2}}{\alpha} \operatorname{sgn}\left(x_{3}-y_{3}\right) e_{\ell}^{\perp} \cdot\{x-y\} \frac{J_{1}(\lambda r)}{r} e_{3} \\
& \left.-e_{\ell}^{\perp} \cdot\{x-y\}\left(\frac{\lambda J_{0}(\lambda r)}{r^{2}}-2 \frac{J_{1}(\lambda r)}{r^{3}}\right)\{x-y\}_{\mathrm{t}}\right) .
\end{aligned}
$$

For $\Theta(x, y)$ and using $J_{0}^{\prime}=-J_{1}$ (Theorem B.3.3) leads to

$$
\operatorname{curl}\left\{\Theta e_{3}\right\}(x, y)=\frac{\lambda^{2}}{\alpha} e^{-\alpha\left|x_{3}-y_{3}\right|} \frac{J_{1}(\lambda r)}{r} e_{3} \times\{x-y\}
$$

and

$$
\begin{aligned}
\operatorname{curl} \operatorname{curl}\left\{\Theta e_{3}\right\}(x, y)= & e^{-\alpha\left|x_{3}-y_{3}\right|}\left(\frac{\lambda^{3}}{\alpha} J_{0}(\lambda r) e_{3}\right. \\
& \left.+\lambda^{2} \operatorname{sgn}\left(x_{3}-y_{3}\right) \frac{J_{1}(\lambda r)}{r}\{x-y\}_{\mathrm{t}}\right) .
\end{aligned}
$$

Note that (6.29)-(6.34) remains valid if everywhere in (6.28)-(6.34) $x_{3}-y_{3}$ is replaced by $x_{3}$.

Now, a representation for the curl of the fundamental solution $\Phi e_{\ell}$ can be formulated.

Lemma 6.2.6 (Representation of the fundamental solution). For $\ell=1,2,3$ and $x_{3} \neq y_{3}$ the following equations holds true

$$
\begin{aligned}
\operatorname{curl}\left\{\Phi_{j} e_{\ell}\right\} & =\operatorname{curl}\left\{F_{\ell} e_{3}\right\}+\operatorname{curl} \operatorname{curl}\left\{G_{\ell} e_{3}\right\} \\
\frac{1}{i \kappa} \operatorname{curl} \operatorname{curl}\left\{\Phi_{j} e_{\ell}\right\} & =\frac{1}{i \kappa} \operatorname{curl} \operatorname{curl}\left\{F_{\ell} e_{3}\right\}-i \kappa \operatorname{curl}\left\{G_{\ell} e_{3}\right\}
\end{aligned}
$$

where the functions $F_{\ell}$ and $G_{\ell}$ are given by

$$
\begin{aligned}
& F_{\ell}(x, y)=\operatorname{sgn}\left(x_{3}-y_{3}\right) \frac{e_{\ell} \cdot\{x-y\}}{4 \pi r} \int_{0}^{\infty} e^{-\alpha\left|x_{3}-y_{3}\right|} J_{1}(\lambda r) \mathrm{d} \lambda, \\
& G_{\ell}(x, y)=\frac{e_{\ell}^{\perp} \cdot\{x-y\}}{4 \pi r} \int_{0}^{\infty} \frac{1}{\alpha} e^{-\alpha\left|x_{3}-y_{3}\right|} J_{1}(\lambda r) \mathrm{d} \lambda
\end{aligned}
$$

for $\ell=1,2$ and

$$
\begin{aligned}
F_{3}(x, y) & =\frac{1}{4 \pi} \int_{0}^{\infty} \frac{\lambda}{\alpha} e^{-\alpha\left|x_{3}-y_{3}\right|} J_{0}(\lambda r) \mathrm{d} \lambda \\
G_{3}(x, y) & =0
\end{aligned}
$$


Proof. First, the case for (6.35) is proven. The proof rests on the Sommerfeld integral (Theorem B.3.6)

$$
\Phi_{j}(x, y)=\frac{1}{4 \pi} \int_{0}^{\infty} \frac{\lambda}{\alpha} e^{-\alpha\left|x_{3}-y_{3}\right|} J_{0}(\lambda r) \mathrm{d} \lambda
$$

that is valid for $x_{3} \neq y_{3}$. The case $\ell=3$ is obvious from (6.39). For the cases $\ell=1,2$ add (6.29) and (6.32) to get

$$
w:=e^{-\alpha\left|x_{3}-y_{3}\right|}\left(\frac{\lambda^{2}}{\alpha} e_{\ell}^{\perp} \cdot\{x-y\} \frac{J_{1}(\lambda r)}{r} e_{3}-\lambda \operatorname{sgn}\left(x_{3}-y_{3}\right) J_{0}(\lambda r) e_{\ell}^{\perp}\right)
$$

On the other hand for $\ell=1,2$ calculate

$$
\begin{aligned}
\operatorname{curl}\left\{\Theta e_{\ell}\right\}(x, y)= & e^{-\alpha\left|x_{3}-y_{3}\right|}\left(-\lambda \operatorname{sgn}\left(x_{3}-y_{3}\right) J_{0}(\lambda r) e_{\ell}^{\perp}\right. \\
& \left.+\frac{\lambda^{2}}{\alpha} e_{\ell}^{\perp} \cdot\{x-y\} \frac{J_{1}(\lambda r)}{r} e_{3}\right)
\end{aligned}
$$

Equations (6.40) and (6.41) are the same and thus can be extended to (6.35). Now, the case of (6.36) follows immediately from (6.35) since the terms individually fulfil the Maxwell equation from (6.28) and are divergence free because of the curls.

Denote by $F_{j, \ell}$ and $G_{j, \ell}$ the functions introduced by (6.37) and (6.38) with $\kappa$ and $\alpha$ replaced by $\kappa_{j}$ and $\alpha_{j}$, respectively. First consider the case where $y \in \mathcal{D}_{1}$. In view of Lemma 6.2.5 it suffices to construct functions $V_{j, \ell}$ and $U_{j, \ell}$ for $j=1,2$ and $\ell=1,2,3$ such that

$$
v_{1, \ell}:=V_{1, \ell}+F_{1, \ell}, \quad v_{2, \ell}:=V_{2, \ell} \quad \text { and } \quad u_{1, \ell}:=U_{1, \ell}+G_{1, \ell}, \quad u_{2, \ell}:=U_{2, \ell}
$$

satisfy the Helmholtz equation with wave numbers $\kappa_{j}$ in $\mathcal{D}_{j} \backslash\{y\}$, the radiation condition and the transmission conditions (6.22) and (6.23). Then for $j=1,2$ the columns of the electric Green's tensor are given through

$$
G_{E, 1} e_{\ell}=\operatorname{curl}\left\{\Phi_{1} e_{\ell}\right\}+\widetilde{G}_{E, 1} e_{\ell}, \quad G_{E, 2} e_{\ell}=\widetilde{G}_{E, 2} e_{\ell},
$$

with

$$
\widetilde{G}_{E, j} e_{\ell}=\operatorname{curl} \operatorname{curl}\left\{U_{j, \ell} e_{3}\right\}+\operatorname{curl}\left\{V_{j, \ell} e_{3}\right\} .
$$

Analogously the columns of the magnetic tensor are given by

$$
G_{H, 1} e_{\ell}=\frac{1}{i \kappa_{j}} \operatorname{curl} \operatorname{curl}\left\{\Phi_{1} e_{\ell}\right\}+\widetilde{G}_{H, 1} e_{\ell}, \quad G_{H, 2} e_{\ell}=\widetilde{G}_{H, 2} e_{\ell},
$$

with

$$
\widetilde{G}_{H, j} e_{\ell}=-i \kappa_{j} \operatorname{curl}\left\{U_{j, \ell} e_{3}\right\}+\frac{1}{i \kappa_{j}} \operatorname{curl} \operatorname{curl}\left\{V_{j, \ell} e_{3}\right\}
$$

The ansatz for the solutions are given in the form

$$
V_{j, \ell}(x, y)=\operatorname{sgn}\left(x_{3}\right) \frac{1}{4 \pi} \frac{e_{\ell} \cdot\{x-y\}}{r} \int_{0}^{\infty} A_{j}(\lambda) e^{-\alpha_{j}\left|x_{3}\right|-\alpha_{1} y_{3}} J_{1}(\lambda r) \mathrm{d} \lambda
$$


and

$$
U_{j, \ell}(x, y)=\frac{1}{4 \pi} \frac{e_{\ell}^{\perp} \cdot\{x-y\}}{r} \int_{0}^{\infty} \frac{B_{j}(\lambda)}{\alpha_{j}} e^{-\alpha_{j}\left|x_{3}\right|-\alpha_{1} y_{3}} J_{1}(\lambda r) \mathrm{d} \lambda
$$

for $\ell=1,2$ and

$$
V_{j, 3}(x, y)=\frac{1}{4 \pi} \int_{0}^{\infty} \frac{\lambda C_{j}(\lambda)}{\alpha_{j}} e^{-\alpha_{j}\left|x_{3}\right|-\alpha_{1} y_{3}} J_{0}(\lambda r) \mathrm{d} \lambda
$$

and $U_{j, 3}=0$ with unknown continuous and integrable functions $A_{j}, B_{j}$ and $C_{j}$. In view of Lemma 6.2.6 and the vector Helmholtz equation the functions $v_{j, \ell}$ and $u_{j, \ell}$ fulfil the Helmholtz equation. Then, Lemma 6.2.5 can be used. The boundary conditions of the lemma lead to inhomogeneous $2 \times 2$ linear systems for the coefficients. For $v_{j, \ell}, j=1,2$ this system is given as

$$
\begin{aligned}
A_{1}-e^{-\alpha_{1} y_{3}} & =-a_{E} A_{2} \\
\frac{\alpha_{1}}{\kappa_{1}}\left(-A_{1}-e^{-\alpha_{1} y_{3}}\right) & =-\frac{\alpha_{2} a_{H}}{\kappa_{2}} A_{2}
\end{aligned}
$$

with the solution

$$
A_{1}=\frac{a_{H} \alpha_{2} \kappa_{1}-a_{E} \alpha_{1} \kappa_{2}}{a_{H} \alpha_{2} \kappa_{1}+a_{E} \alpha_{1} \kappa_{2}} e^{-\alpha_{1} y_{3}}, \quad A_{2}=\frac{2 \alpha_{1} \kappa_{2}}{a_{H} \alpha_{2} \kappa_{1}+a_{E} \alpha_{1} \kappa_{2}} e^{-\alpha_{1} y_{3}}
$$

For $u_{j, \ell}, j=1,2$ the linear system has the form

$$
\begin{aligned}
\frac{\kappa_{1}}{\alpha_{1}}\left(B_{1}+e^{-\alpha_{1} y_{3}}\right) & =\frac{\kappa_{2} a_{H}}{\alpha_{2}} B_{2} \\
-B_{1}+e^{-\alpha_{1} y_{3}} & =a_{E} B_{2}
\end{aligned}
$$

and the solution turns out to be

$$
B_{1}=\frac{a_{H} \kappa_{2} \alpha_{1}-a_{E} \kappa_{1} \alpha_{2}}{a_{H} \kappa_{2} \alpha_{1}+a_{E} \mathcal{\kappa}_{1} \alpha_{2}} e^{-\alpha_{1} y_{3}}, \quad B_{2}=\frac{2 \kappa_{1} \alpha_{2}}{a_{H} \kappa_{2} \alpha_{1}+a_{E} \kappa_{1} \alpha_{2}} e^{-\alpha_{1} y_{3}}
$$

For $v_{j, 3}$ the system is

$$
\begin{aligned}
\frac{1}{\alpha_{1}}\left(C_{1}+e^{-\alpha_{1} y_{3}}\right) & =\frac{a_{E}}{\alpha_{2}} C_{2} \\
\frac{1}{\kappa_{1}}\left(-C_{1}+e^{-\alpha_{1} y_{3}}\right) & =\frac{a_{H}}{\kappa_{2}} C_{2}
\end{aligned}
$$

with the solution

$$
C_{1}=-\frac{a_{H} \alpha_{2} \kappa_{1}-a_{E} \alpha_{1} \kappa_{2}}{a_{H} \alpha_{2} \kappa_{1}+a_{E} \alpha_{1} \kappa_{2}} e^{-\alpha_{1} y_{3}}, \quad C_{2}=\frac{2 \alpha_{2} \kappa_{2}}{a_{H} \alpha_{2} \kappa_{1}+a_{E} \alpha_{1} \kappa_{2}} e^{-\alpha_{1} y_{3}}
$$


Theorem 6.2.7 (Green's tensor). For $y \in \mathcal{D}_{1}$ the columns of the electric field in the Green's tensor are given by

$$
\begin{aligned}
& \widetilde{G}_{E, j}(x, y) e_{\ell}=\frac{e_{\ell}^{\perp} \cdot\{x-y\}}{4 \pi r} e_{3} \int_{0}^{\infty} B_{j}(\lambda) \frac{\lambda^{2}}{\alpha_{j}} e^{-\alpha_{j}\left|x_{3}\right|} J_{1}(\lambda r) \mathrm{d} \lambda \\
& \quad-\operatorname{sgn}\left(x_{3}\right) \frac{1}{4 \pi r} e_{\ell}^{\perp} \int_{0}^{\infty}\left[A_{j}(\lambda)+B_{j}(\lambda)\right] e^{-\alpha_{j}\left|x_{3}\right|} J_{1}(\lambda r) \mathrm{d} \lambda \\
& \quad+\operatorname{sgn}\left(x_{3}\right) \frac{e_{\ell} \cdot\{x-y\}}{4 \pi r}\{x-y\} \times e_{3} \int_{0}^{\infty} A_{j}(\lambda) e^{-\alpha_{j}\left|x_{3}\right|}\left(\frac{\lambda J_{0}(\lambda r)}{r}-2 \frac{J_{1}(\lambda r)}{r^{2}}\right) \mathrm{d} \lambda \\
& \quad-\operatorname{sgn}\left(x_{3}\right) \frac{e_{\ell}^{\perp} \cdot\{x-y\}}{4 \pi r}\{x-y\}_{t} \int_{0}^{\infty} B_{j}(\lambda) e^{-\alpha_{j}\left|x_{3}\right|}\left(\frac{\lambda J_{0}(\lambda r)}{r}-2 \frac{J_{1}(\lambda r)}{r^{2}}\right) \mathrm{d} \lambda
\end{aligned}
$$

for $\ell=1,2$ and

$$
\widetilde{G}_{E, j}(x, y) e_{3}=\frac{1}{4 \pi r} e_{3} \times\{x-y\} \int_{0}^{\infty} C_{j}(\lambda) \frac{\lambda^{2}}{\alpha_{j}} e^{-\alpha_{j}\left|x_{3}\right|} J_{1}(\lambda r) \mathrm{d} \lambda .
$$

The columns of the Green's tensor for the magnetic field are given by

$$
\begin{aligned}
\widetilde{G}_{H, j}(x, y) e_{\ell}=\operatorname{sgn}\left(x_{3}\right) \frac{e_{\ell}^{\perp} \cdot\{x-y\}}{4 \pi i \kappa_{j} r} e_{3} \int_{0}^{\infty} A_{j}(\lambda) \lambda^{2} e^{-\alpha_{j}\left|x_{3}\right|} J_{1}(\lambda r) \mathrm{d} \lambda \\
-\frac{1}{4 \pi i \kappa_{j} r} e_{\ell}^{\perp} \int_{0}^{\infty} \frac{1}{\alpha_{j}}\left[\alpha_{j}^{2} A_{j}(\lambda)-\kappa_{j}^{2} B_{j}(\lambda)\right] e^{-\alpha\left|x_{3}\right|} J_{1}(\lambda r) \mathrm{d} \lambda \\
+\frac{e_{\ell} \cdot\{x-y\}}{4 \pi i \kappa_{j} \alpha_{j} r}\{x-y\} \times e_{3} \int_{0}^{\infty} \frac{\kappa_{j}^{2}}{\alpha_{j}} B_{j}(\lambda) e^{-\alpha_{j}\left|x_{3}\right|}\left(\frac{\lambda J_{0}(\lambda r)}{r}-2 \frac{J_{1}(\lambda r)}{r^{2}}\right) \mathrm{d} \lambda \\
-\frac{e_{\ell}^{\perp} \cdot\{x-y\}}{4 \pi i \kappa_{j} r}\{x-y\}_{t} \int_{0}^{\infty} \alpha_{j} A_{j}(\lambda) e^{-\alpha_{j}\left|x_{3}\right|}\left(\frac{\lambda J_{0}(\lambda r)}{r}-2 \frac{J_{1}(\lambda r)}{r^{2}}\right) \mathrm{d} \lambda
\end{aligned}
$$

for $\ell=1,2$ and

$$
\begin{aligned}
\widetilde{G}_{H, j}(x, y) e_{3}= & \operatorname{sgn}\left(x_{3}\right) \frac{1}{4 \pi i \kappa_{j} r}\{x-y\}_{t} \int_{0}^{\infty} C_{j}(\lambda) \lambda^{2} e^{-\alpha\left|x_{3}\right|} J_{1}(\lambda r) \mathrm{d} \lambda \\
& +\frac{1}{4 \pi i \kappa_{j} \alpha_{j}} e_{3} \int_{0}^{\infty} C_{j}(\lambda) \frac{\lambda^{3}}{\alpha_{j}} e^{-\alpha\left|x_{3}\right|} J_{0}(\lambda r) \mathrm{d} \lambda
\end{aligned}
$$

Proof. From the ansatz functions $V_{j, \ell}(6.44), U_{j, \ell}(6.45)$ and $V_{3, \ell}(6.46)$ the tensor for the electric field follows from (6.29), (6.32) and (6.33) and the tensor for the magnetic field follows from (6.30), (6.31) and (6.34).

Finally consider the case where $y \in \mathcal{D}_{2}$. Here construct functions $V_{j, \ell}$ and $U_{j, \ell}$ for $j=1,2$ and $\ell=1,2,3$ such that

$$
v_{1, \ell}:=V_{1, \ell}, \quad v_{2, \ell}:=V_{2, \ell}+F_{1, \ell} \quad \text { and } \quad u_{1, \ell}:=U_{1, \ell}, \quad u_{2, \ell}:=U_{2, \ell}+G_{1, \ell}
$$


satisfy the Helmholtz equation with wave numbers $\kappa_{j}$ in $\mathcal{D}_{j} \backslash\{y\}$, the radiation condition and the transmission conditions (6.22) and (6.23). Then the Green's tensor is given through

$$
G_{E, 1} e_{\ell}=\widetilde{G}_{E, 1} e_{\ell}, \quad G_{E, 2} e_{\ell}=\operatorname{curl}\left\{\Phi_{1} e_{\ell}\right\}+\widetilde{G}_{E, 2} e_{\ell},
$$

with $\widetilde{G}_{E, j}$ as in (6.42). The corresponding linear systems lead to the coefficients

$$
A_{1}=\frac{2 \alpha_{2} \kappa_{1} a_{E} a_{H}}{a_{H} \alpha_{2} \kappa_{1}+a_{E} \alpha_{1} \kappa_{2}} e^{\alpha_{2} y_{3}}, \quad A_{2}=-\frac{a_{H} \alpha_{2} \kappa_{1}-a_{E} \alpha_{1} \kappa_{2}}{a_{H} \alpha_{2} \kappa_{1}+a_{E} \alpha_{1} \kappa_{2}} e^{\alpha_{2} y_{3}}
$$

replacing (6.47),

$$
B_{1}=\frac{2 \kappa_{2} \alpha_{1} a_{H} a_{E}}{a_{H} \kappa_{2} \alpha_{1}+a_{E} \kappa_{1} \alpha_{2}} e^{\alpha_{2} y_{3}}, \quad B_{2}=-\frac{a_{H} \kappa_{2} \alpha_{1}-a_{E} \kappa_{1} \alpha_{2}}{a_{H} \kappa_{2} \alpha_{1}+a_{E} \kappa_{1} \alpha_{2}} e^{\alpha_{2} y_{3}} .
$$

replacing (6.48) and

$$
C_{1}=\frac{2 \alpha_{1} \kappa_{1} a_{H} a_{E}}{a_{H} \alpha_{2} \kappa_{1}+a_{E} \alpha_{1} \kappa_{2}} e^{\alpha_{2} y_{3}}, \quad C_{2}=\frac{a_{H} \alpha_{2} \kappa_{1}-a_{E} \alpha_{1} \kappa_{2}}{a_{H} \alpha_{2} \kappa_{1}+a_{E} \alpha_{1} \kappa_{2}} e^{\alpha_{2} y_{3}}
$$

replacing (6.49). Note that this corresponds to replacing $e^{-\alpha_{1} y_{3}}$ by $e^{\alpha_{2} y_{3}}$ and interchanging the roles of the two half spaces.

\subsubsection{Surface potentials}

Like in the acoustic case, surface potentials are introduced following Huygens principle. In the $3 \mathrm{D}$ environment needed for electromagnetics densities which are tangential to a boundary are needed. This spaces are defined and the question how a gradient and a divergence can be understood for boundaries is answered.

Definition 6.2.8 (surface gradient/divergence). Let

$$
x(u)=\left(x_{1}\left(u_{1}, u_{2}\right), x_{2}\left(u_{1}, u_{2}\right), x_{3}\left(u_{1}, u_{2}\right)\right)
$$

be a parametric representation of a surface patch of $\partial D$. Then the surface gradient is given by

$$
\operatorname{Grad} \varphi=\sum_{i, j=1}^{2} g_{i j}^{-1} \frac{\partial \varphi}{\partial u_{i}} \frac{\partial x}{\partial u_{j}}
$$

with the first fundamental matrix of differential geometry

$$
g_{i j}:=\frac{\partial x}{\partial u_{i}} \cdot \frac{\partial x}{\partial u_{j}} \quad i, j=1,2 .
$$

An integrable tangential vector field $a$ has a weak surface divergence if there exists an integrable scalar denoted by (Div $a)$ such that

$$
\int_{\partial D} \varphi \operatorname{Div} a \mathrm{~d} s=-\int_{\partial D} \operatorname{Grad} \varphi \cdot a \mathrm{~d} s
$$

is satisfied for all $\varphi \in C^{1}(\partial D)$. Then, the identity

$$
\operatorname{Div}\left(v \times E_{j}\right)=-v \cdot \operatorname{curl} E_{j}
$$

holds true. 
The space of tangential fields which are continuous are denoted by $C_{t}(\partial D)$. Then, let the normed spaces of tangential fields possessing a surface divergence be defined as

$$
C_{t, d}(\partial D):=\left\{a \in C_{t}(\partial D): \operatorname{Div} a \in C(\partial D)\right\}
$$

equipped with the norm

$$
\|a\|_{C_{t, d}}:=\|a\|_{\infty}+\|\operatorname{Div} a\|_{\infty}
$$

Let the space of Hölder continuous tangential vector fields to $\partial D$ denoted by $C_{t}^{0, \alpha}(\partial D)$. Then the subspace of all fields possessing a Hölder continuous surface divergence is defined as the normed space

$$
C_{t, d}^{0, \alpha}(\partial D):=\left\{a \in C_{t}^{0, \alpha}(\partial D): \text { Div } a \in C^{0, \alpha}(\partial D)\right\}
$$

equipped with the norm

$$
\|a\|_{C_{t, d}^{0, \alpha}}:=\|a\|_{0, \alpha}+\|\operatorname{Div} a\|_{0, \alpha}
$$

Definition 6.2.9 (Vector surface Potentials). Let $a \in L^{1}(\partial D)$ be an integrable vector field on the boundary $\partial D$. Then, the integral

$$
A(x):=\int_{\partial D} \Phi(x, y) a(y) \mathrm{d} s(y), \quad x \in \mathbb{R}^{3} \backslash \partial D
$$

is called the vector surface potential with density $a$.

In the same way the jump relations are extended from the scalar to the vector case.

Theorem 6.2.10 (Jump relation vector surface potential). Let $\partial D$ be of Class $C^{2}$ and let $a \in C_{t}(\partial D)$ be a continuous tangential field. Then the vector potential $A$ with density $a \in C_{t, d}$ is continuous throughout $\mathbb{R}^{3}$. On the boundary the limiting values are given by

$$
\begin{aligned}
A(x) & =\int_{\partial D} a(y) \Phi(x, y) \mathrm{d} s(y) \\
v(x) \times \operatorname{curl} A_{ \pm}(x) & =\int_{\partial D} v(x) \times \operatorname{curl}_{x} a(y) \Phi(x, y) \mathrm{d} s(y) \pm \frac{1}{2} a(x) \\
v(x) \times \operatorname{curl} \operatorname{curl} A_{ \pm}(x) & =v(x) \times \kappa^{2} A(x)+v \times \operatorname{grad} \operatorname{div} A(x)
\end{aligned}
$$

for $x \in \partial D$ where

$$
\begin{aligned}
v(x) \times \operatorname{curl} A_{ \pm}(x) & :=\lim _{h \rightarrow+0} v(x) \times \operatorname{curl} A(x \pm h v(x)) \\
v(x) \times \operatorname{curl} \operatorname{curl} A_{ \pm}(x) & :=\lim _{h \rightarrow+0} v(x) \times \operatorname{curl} \operatorname{curl} A(x \pm h v(x))
\end{aligned}
$$

is to be understood in the sense of uniform convergence on $\partial D$ and where the integrals exist as improper integrals. This implies

$$
\left\langle\nu, \operatorname{curl} \operatorname{curl} A_{+}-\operatorname{curl} \operatorname{curl} A_{-}\right\rangle=0
$$


Proof. See proof of Theorem 6.11 of [8] and using the identity (6.7) leads to the third equation of (6.53).

For later use in the integral methods define the following integral operators.

Definition 6.2.11 (magnetic dipole operator). The magnetic dipole operator is defined as the integral operator $M_{j}: C_{t, d}^{0, \alpha}(\partial D) \mapsto C_{t, d}^{0, \alpha}(\partial D)$ given by

$$
\left(M_{j} a\right)(x):=2 v(x) \times \int_{\partial D} G_{E, j}(x, y) a(y) \mathrm{d} s(y), \quad x \in \partial D,
$$

for $j=0,2,3$ and whereas the case $j=3$ and $j=0$ only differ in the value of $\kappa$.

Remark. The operator for $j=0,3$ is a compact operator (see Theorem 6.15 and 6.16 from [8]). Since the operator for $j=2$ differs from the corresponding integral operator for the free space problem $j=0$ only by an integral operator with a smooth kernel, again from Theorem 6.16 and 6.15 in [8] it follows that $M_{j}, j=2$ is a compact operator.

Definition 6.2.12 (electric dipole operator). The electric dipole operator for the full space $N_{j}: C_{t, d}^{0, \alpha}(\partial D) \mapsto C_{t, d}^{0, \alpha}(\partial D)$ is given by

$$
\left(N_{0} a\right)(x):=2 v(x) \times \text { curlcurl } \int_{\partial D} v(y) \times \Phi_{0}(x, y) a(y) \mathrm{d} s(y), \quad x \in \partial D .
$$

For the transmission problem the operators are given by

$$
\begin{gathered}
\left(N_{0, t} a\right)(x):=2 v(x) \times \text { curlcurl } \int_{\partial D} \Phi_{0}(x, y) a(y) \mathrm{d} s(y), \quad x \in \partial D, \\
\left(N_{2} a\right)(x)=2 v(x) \times \text { curl curl } \int_{\partial D} \Phi_{2}(x, y) a(y) \mathrm{d} s(y) \\
+2 v(x) \times \int_{\partial D} \widetilde{G}_{H, 2}(x, y) a(y) \mathrm{d} s(y), \quad x \in \partial D,
\end{gathered}
$$

and

$$
\left(N_{3} a\right)(x):=2 v(x) \times \operatorname{curl} \text { curl } \int_{\partial D} \Phi_{3}(x, y) a(y) \mathrm{d} s(y), \quad x \in \partial D .
$$

Remark. The operator for the full space is a bounded operator (see Theorem 6.15 and 6.17 from [8]). The operators $\left(N_{2}-N_{3}\right)$ are compact operators (see [9, Theorem 2.33] ) .

\subsection{Common settings for mine detection}

In the application of mine detection there are some special settings for the domains, parameters and measurements. In particular for the simulation of handheld detectors the given situation can be defined as follows. 
Definition 6.3.1 (Handheld mine detection). Let the mine detector be composed of an emitter and receiver loop which are linked together in a static chassis. The handheld detector operates in the air and tries to find metallic objects (especially mines) beneath the ground. The environment can roughly be seperated into two layers, the air and the earth. The conductivity $\sigma$ in the air is assumed to be 0 and in the earth $\sigma$ is assumed to be greater then 0 .

The mine detector scans through an roughly plane area $\mathcal{M}$ in a specific height above the ground. For every position $x \in \mathcal{M}$ the emitter sends out the incident electromagnetic field $E^{i}$, which is scattered by the buried objects. The scattered field $E^{s}$ from the objects induces a voltage inside the receiver loop, which is then measured as induced Voltage $U$.

The source (Section 6.3.1), the measurement (Section 6.3.2) and the physical constants (Section 6.3.3) are examined in more detail in the following subsections.

\subsubsection{The source}

In the setting at hand the following two forms of sources are used. For the range test later on also plane waves are used, which are introduced there.

Definition 6.3.2 (Magnetic and electric dipole). The incident field could by given by a magnetic dipole

$$
E_{j}^{i, m}(x):=G_{E, j}(x, y) p, \quad H_{j}^{i, m}(x):=\frac{1}{i \kappa_{j}} \operatorname{curl} E_{j}^{i, m}(x)
$$

or an electric dipole

$$
H_{j}^{i, e}(x):=G_{E, j}(x, y) p, \quad E_{j}^{i, e}(x):=-\frac{1}{i \kappa_{j}} \operatorname{curl} H_{j}^{i, e}(x)
$$

for $j=0,1,2$ with polarisation $p \in \mathbb{R}^{3}$.

Definition 6.3.3 (Wire loop). In the mine detection application the incident field is generated by some time-depending current $I(t)$ in a wire loop $\Gamma_{s}$ contained in $D_{1}$ (see Figure 6.3). The magnetic field $\mathcal{H}_{j}(x, t)$ of a time-harmonic current $I(t)=I_{0} \Re e^{-i \omega t}$ on $\Gamma_{s}$ is given via Biot-Savart's law by

$$
\mathcal{H}_{j}(x, t)=\Re\left\{I_{0} \int_{\Gamma_{s}} G_{E, j}(x, y) \cdot \tau(y) e^{-i \omega t} \mathrm{~d} s(y)\right\}, \quad x \in D_{j},
$$

where $\tau$ denotes the unit tangent vector to $\Gamma_{s}$.

By the Maxwell equations (6.4) and the time harmonic representation (6.2) this leads to the space dependent incident fields

$$
H_{j}^{i}(x)=I_{0} \sqrt{\mu_{j}} \int_{\Gamma_{s}} G_{E, j}(x, y) \cdot \tau(y) \mathrm{d} s(y) x \in D_{j} \backslash\{y\}
$$




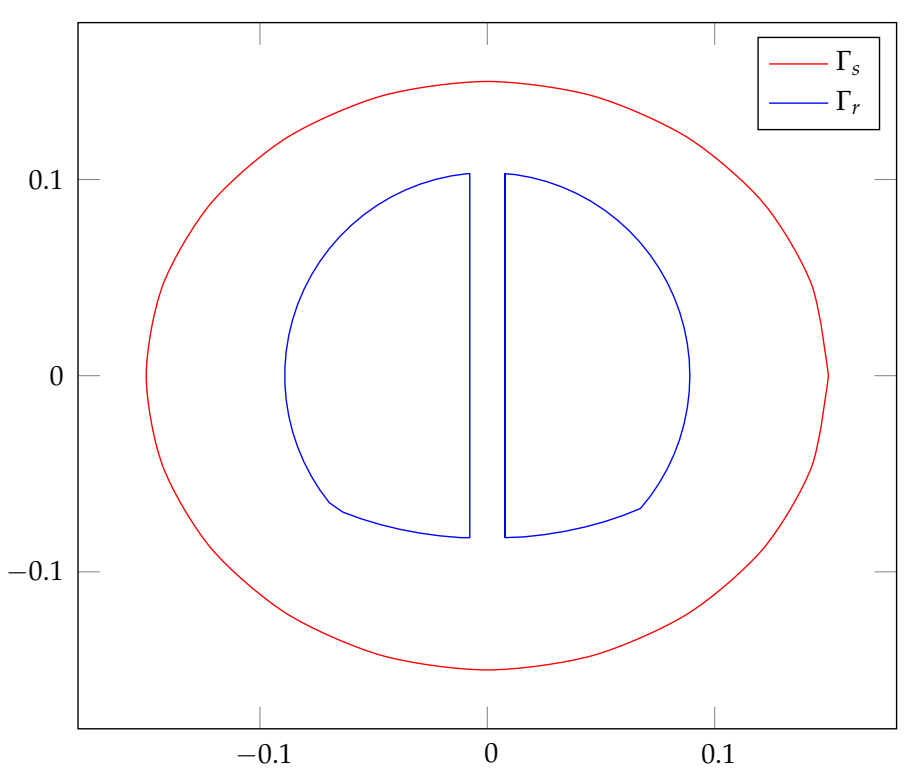

Figure 6.3: Wire loop of source $\Gamma_{s}$ and receiver $\Gamma_{r}$. The receiver loop is an imitation of the loop in the Foerster MINEX 2 FD 4.500 handheld mine detector.

Again by (6.4) this becomes

$$
E_{j}^{i}(x)=-\frac{1}{i \kappa_{j}} \operatorname{curl} H_{j}^{i}(x)=-I_{0} \sqrt{\mu_{j}} \int_{\Gamma_{s}} G_{H, j}(x, y) \cdot \tau(y) \mathrm{d} s(y) x \in D_{j} \backslash\{y\} .
$$

The fields in (6.63) and (6.62) are used later on as incident field $E_{j}^{i}, H_{j}^{i}$.

\subsubsection{The measurement}

Measurements in mine detectors are carried out by a secondary wire loop $\Gamma_{r}$, which often has the form of a "double $\mathrm{D}^{\prime}$ (see Figure 6.3). The induction voltage in the measurement loop $\Gamma_{r}$ enclosing the area $A_{\Gamma_{r}}$ is given via Faraday's law

$$
\mathcal{U}_{j}(t)=\Re \int_{\Gamma_{r}} \mathcal{E}_{j}^{s}(x, t) \cdot \tau(x) \mathrm{d} s(x),
$$

that is,

$$
\mathcal{U}_{j}(t)=\Re \int_{\Gamma_{r}}\left\{\left(\epsilon_{j}+\frac{i \sigma_{j}}{\omega}\right)^{-1 / 2} E_{j}^{s}(x) e^{-i \omega t}\right\} \cdot \tau(x) \mathrm{d} s(x)
$$

where $\tau$ denotes the unit tangent vector to $\Gamma_{r}$. This implies

$$
\mathcal{U}_{j}(t)=\Re\left\{\left(\epsilon_{j}+\frac{i \sigma_{j}}{\omega}\right)^{-1 / 2} U_{j} e^{-i \omega t}\right\}
$$

with

$$
U_{j}=\int_{\Gamma_{r}} E_{j}^{s}(x) \cdot \tau(x) \mathrm{d} s(x)
$$




\begin{tabular}{lc}
\hline Constant & {$[\mathrm{As} / \mathrm{Vm}]$} \\
\hline$\epsilon_{0}$ & $8.8542 \cdot 10^{-12}$ \\
$\mu_{0}$ & $1.2566 \cdot 10^{-6}$ \\
\hline
\end{tabular}

Table 6.1: Fundamental physical constants.

\begin{tabular}{lcccc}
\hline Material or medium & $\epsilon_{r}$ & $\sigma[\mathrm{A} / \mathrm{Vm}]$ & $\mu_{r}$ & $\kappa\left[\mathrm{m}^{-1}\right]$ \\
\hline Aluminum (Al) & 1.7 & $37.8 \cdot 10^{6}$ & $1+2.22 \cdot 10^{-5}$ & $598(1+i)$ \\
Copper (Cu) & 2.8 & $59.6 \cdot 10^{6}$ & $1-6.4 \cdot 10^{-6}$ & $747(1+i)$ \\
Air & 1.00054 & $\mathrm{O}$ & 1 & $5.03 \cdot 10^{-5}$ \\
Conducting ground & 4 & 0.05 & 1 & $2.18 \cdot 10^{-2}(1+i)$ \\
\hline
\end{tabular}

Table 6.2: Example for some material constants.

\subsubsection{Physical constants}

First, the fundamental physical constants used here are the electric permittivity in vacuum $\epsilon_{0}$ and the magnetic permeability in vacuum $\mu_{0}$ which are shown in Table 6.1. A useful quantity is then the relative electric permittivity $\epsilon_{r}$ and relative magnetic permeability $\mu_{r}$

$$
\epsilon_{j}=\epsilon_{r, j} \epsilon_{0}, \quad \mu_{j}=\mu_{r, j} \mu_{0}
$$

which gives the total values inside the domains $D_{j}, j=0,1,2,3$.

Typical mine detectors use frequencies between $300 \mathrm{~Hz}$ and $1 \mathrm{MHz}$. This results in $\kappa$ being between $\kappa_{1} \approx 5 \cdot 10^{-5} \mathrm{~m}^{-1}$ for the air or low conducting ground and low frequency and $\kappa_{2} \approx 3(1+\mathrm{i}) \mathrm{m}^{-1}$ for a frequency of $1 \mathrm{MHz}$ and high conductivity of the soil. Two examples of potential values of $\kappa$ are shown in the following. For a frequency of $1 \mathrm{kHz}$ and $\epsilon_{r}=10, \mu_{r}=1$ and $\sigma=0 \mathrm{~A} / \mathrm{Vm}$ we obtain $\kappa=7 \cdot 10^{-5} \mathrm{~m}^{-1}$. For $\sigma=10^{-5}$ the wave number becomes $\kappa=3 \cdot 10^{-4} \mathrm{~m}^{-1}$. The values which are mainly used in the numerical examples in this work are given by

$$
\kappa_{1} \approx 5 \cdot 10^{-5} \mathrm{~m}^{-1}, \quad \kappa_{2} \approx 10^{-2}(1+\mathrm{i}) \mathrm{m}^{-1} \text {, }
$$

which corresponds to using a frequency of $\omega=2400 \mathrm{~Hz}$ and the material constants $\sigma_{1}=0 \mathrm{~A} / \mathrm{Vm}, \epsilon_{r, 1}=1, \mu_{r, 1}=1$ for the upper halfspace (air) and $\sigma_{2}=$ $0.05 \mathrm{~A} / \mathrm{Vm}, \epsilon_{r, 2}=4, \mu_{r, 2}=1+1.825 \cdot 10^{-5}$ for the lower halfspace (soil). In Table 6.2 some used and common constants are summed up. 


\section{Chapter 7}

\section{Direct Problem}

The direct problems under consideration are three-dimensional electromagnetic scattering problems. In particular, in this work four different direct problems are examined.

First, the setting common for all four problems is described. Let the domain $D \subset D_{2}$ has a sufficiently smooth boundary, i.e. assume a $C^{2}$-boundary and let a measurement area be denoted as $\mathcal{M} \in D_{1}$, a typical area is a plane some centimetres above the ground. Then, an incoming wave $E_{j}^{i}, H_{j}^{i}$ scatters on the obstacle $D$ which then radiates outwards as scattered wave $E_{j}^{s}, H_{j}^{s}$, i.e. the electromagnetic fields are decomposed into the primary fields $E_{j}^{i} H_{j}^{i}$ and the secondary fields $E_{j}^{s}, H_{j}^{s}$. The primary field is generated by an induction current in a wire loop, compare Figure 6.3, or by single point sources. The total fields

$$
E_{j}=E_{j}^{s}+E_{j}^{i}, \quad H_{j}=H_{j}^{s}+H_{j}^{i}
$$

satisfy Maxwell's equations (6.4) (outside the wire loop), and, depending on the four cases, transmission and/or boundary conditions (Section 6.1.2) and (Section 6.1.3). Further, the scattered waves fulfil the radiation conditions (Section 6.1.4). The four different background settings which defines the four different problems are summed up in the following Definition.

Definition 7.0.4 (Four direct problems). The domain and background settings for the four cases of direct problems are given in the following list.

(HP) Homogeneous background medium with perfectly conducting obstacle.

(LP) Layered background medium with perfectly conducting obstacle.

(HT) Homogeneous background medium with a homogenous conducting obstacle.

(LT) Layered background medium with a homogeneous conducting obstacle.

For the reality of mine detection the best possible case is (LT), i.e. an metallic obstacle in a two-layered background medium. 


\subsection{Perfect conductor in a homogenous background medium}

Here, as a first step towards the full layered-medium problem, the approach for a homogeneous background medium is described.

The solution for problem in a homogenous medium $\mathbb{R}^{3}$ is based on a boundary integral equation method. The electric and magnetic field in the full space is influenced both by the background medium and the metallic scatterers. For simplicity, the index $j=0$ for the full space is neglected in the whole section

Definition 7.1.1 (Direct scattering problem (HP)). With given incident time-harmonic electromagnetic field $E^{i}, H^{i}$, find two complex-valued vector fields $E^{s}, H^{s} \in C^{1}\left(\mathbb{R}^{3} \backslash\right.$ $\bar{D})$. Then, the incident fields, the scattered fields and the total time-harmonic electromagnetic field satisfy the time harmonic Maxwell equations (6.4)

$$
\begin{aligned}
& \operatorname{curl} E(x)-i \kappa H(x)=0 \\
& \operatorname{curl} H(x)+i \kappa E(x)=0
\end{aligned}
$$

for $x \in \mathbb{R}^{3} \backslash \bar{D}$. The scattered fields satisfy the Silver-Müller radiation condition (6.11) and the total field satisfy the boundary condition (6.9).

\subsubsection{Uniqueness}

Theorem 7.1.2 (Uniqueness (HP)). The scattering problem for a perfect conductor in a homogeneous background medium (Definition 7.1.1) has at most one solution.

Proof. Let $E^{s, d}=E^{s, 1}-E^{s, 2}$ and $H^{s, d}=H^{s, 1}-H^{s, 2}$ be the difference of two solutions with the same incident field $\left(E^{i}, H^{i}\right)$. Then $\left(E^{s, d}, H^{s, d}\right)$ satisfies the Maxwell's equations (6.4), boundary condition (6.9) and the radiation condition (6.11).

The boundary condition (6.9) implies directly

$$
\Re \int_{\partial D} v \times E^{s, d} \cdot \bar{H}^{s, d} \mathrm{~d} s=0
$$

which is an assumption for the extended Rellich's Lemma (Lemma 6.1.4). Now applying the extended Rellich's Lemma leads to $E^{s, d}=H^{s, d}=0$ in $\mathbb{R}^{3} \backslash \bar{D}$, which completes the proof.

\subsubsection{Existence}

With the fundamental solution (Definition 2.2.1) the following operators can be defined. Let $a \in C_{t, d}^{0, \alpha}(\partial D)$ be a tangential field and $\eta \neq 0$ a real coupling parameter. Further, the single layer operator (compare Definition 2.2.2) in the potential theoretic case of $\kappa=0$ is given by

$$
\left(S_{0} a\right):=2 \int_{\partial D} a(y) \Phi(x, y) \mathrm{d} s(y), x \in \partial D,
$$


which is a compact operator from $C^{0, \alpha}(\partial D)$ into $C^{0, \alpha}(\partial D)$. Then, with the magnetic and electric dipole operator (Definition 6.2.11 and Definition 6.2.12) the ansatz for $E^{S}, H^{S} \in \mathbb{R}^{3} \backslash \bar{D}$ with a combined single- and double-layer potential is given by

$$
\begin{aligned}
E^{S}(x) & =\left(P_{1} a\right)(x)+i \eta\left(P_{2} a\right)(x) \\
H^{S}(x) & =\frac{1}{i \kappa} \operatorname{curl} E^{S}(x),
\end{aligned}
$$

with the operators

$$
\begin{aligned}
& \left(P_{1} a\right)(x):=\operatorname{curl} \int_{\partial D} a(y) \Phi(x, y) \mathrm{d} s(y), x \in \mathbb{R}^{3} \backslash \partial D, \\
& \left(P_{2} a\right)(x):=\text { curl curl } \int_{\partial D} v(y) \times\left(S_{0}^{2} a\right)(y) \Phi(x, y) \mathrm{d} s(y), \quad x \in \mathbb{R}^{3} \backslash \partial D .
\end{aligned}
$$

Using the vector Helmholtz equation (Theorem 6.1.2), the equivalence of the radiation conditions (Theorem 6.1.3) and the jump relations (Theorem 6.2.10) the fields $E^{S}, H^{s}$ defined in (7.3) solve the direct scattering problem (7.1.1) provided the density a solves the integral equation

$$
a+M_{0} a+i \eta N_{0} P S_{0}^{2} a=-2 v \times E^{i}
$$

where $P$ is the projection operator $P b:=(v \times b) \times v$ mapping a density on the boundary to the tangent plane, $M_{0}$ the magnetic dipole operator (Definition 6.2.11) and $N_{0}$ the electric dipole operator (Definition 6.2.12).

Remark. This combined approach is chosen because for real $\kappa$ with $\eta=0$ the solution to the integral equation could have eigenvalues for specific wave numbers $\kappa$, i.e. the integral equation then would have nonunique solutions.

With this ansatz the existence can be proven. For a constant background medium the following existence theorem is well known.

Theorem 7.1.3 (Existence (HP)). The scattering problem (Definition 7.1.1) has a unique solution. Further, the operator which maps the boundary data onto the solution is continuous from $C_{t, d}^{0, \alpha}(\partial D)$ into $C^{0, \alpha}\left(\mathbb{R}^{3} \backslash D\right) \times C^{0, \alpha}\left(\mathbb{R}^{3} \backslash D\right)$.

Proof. The proof follows the proof of [8, Theorem 6.19]). First examine the compactness of the boundary integral operator. The operator $P S_{0}: C^{0, \alpha}(\partial D) \mapsto C_{t, r}^{0, \alpha}(\partial D)$ can be seen to be bounded and $S_{0}$ is a compact operator (see Theorems 3.2, 3.3 and 3.4 from [8]). This implies that $P S_{0}^{2}$ is a compact operator. Then, using the mapping properties of $M_{0}$ and $N_{0}$ the operator

$$
M_{0}+i \eta N_{0} P S_{0}^{2}: C_{t, d}^{0, \alpha}(\partial D) \mapsto C_{t, d}^{0, \alpha}(\partial D)
$$

is a compact operator. Hence the Riesz-Fredholm theory (Theorem A.2.2) is applicable and establishes the existence of $(7 \cdot 5)$, if the operator $M_{0}+i \eta N_{0} P S_{0}^{2}$ (7.6) is injective. Consider the homogeneous form of $(7 \cdot 5)$. Then, from the uniqueness theorem it follows that $E=H=0$ in $\mathbb{R}^{3} \backslash \bar{D}$. From the identity

$$
\operatorname{curl} \operatorname{curl} E=-\Delta E+\operatorname{div} \operatorname{grad} E
$$


it follows that for surface vector potentials $A$ it holds

$$
\operatorname{curl} \operatorname{curl} A(x)=\kappa^{2} \int_{\partial D} a(y) \Phi_{0}(x, y) \mathrm{d} s(y)+\operatorname{grad} \int_{\partial D} \operatorname{Div} a(y) \Phi_{0}(x, y) \mathrm{d} s(y)
$$

on the boundary $\partial D$ and therefore

$$
\operatorname{curl} \operatorname{curl} P_{1}(x)=\kappa^{2} \operatorname{curl} \int_{\partial D} a(y) \Phi_{0}(x, y) \mathrm{d} s(y)
$$

Then, looking at the jump on the boundary using the jump relations (Theorem 6.2.10) leads to

$$
-v \times E_{-}=a, \quad-v \times \operatorname{curl} E_{-}=i \eta \kappa^{2} v \times S_{0}^{2} a .
$$

Now using Gauss' divergence theorem yields

$$
\begin{gathered}
i \eta \kappa^{2} \int_{\partial D}\left|S_{0} a\right|^{2} \mathrm{~d} s=i \eta \kappa^{2} \int_{\partial D} \bar{a} \cdot S_{0}^{2} a \mathrm{~d} s \\
=\int_{\partial D} v \times \bar{E}_{-} \cdot \operatorname{curl} E_{-} \mathrm{d} s=\int_{D}\left\{|\operatorname{curl} E|^{2}-\kappa^{2}|E|^{2}\right\},
\end{gathered}
$$

from which $S_{0} a=0$ follows by taking the imaginary part. The cartesian components of the Vector potential $w$ corresponding to the operator $S_{0}$ with wave number $\kappa=0$ and density $a$ are continuous throughout $\mathbb{R}^{3}$, harmonic in $\mathbb{R}^{3} \backslash \partial D$ and in $D$ and vanish on $\partial D$ and at infinity. Therefore, by the maximum-minimum principle for harmonic functions, it follows that $w=0$ in $\mathbb{R}^{3}$ and the second scalar jump relation (Theorem 2.2.4) yields $a=0$. Thus, injectivity of the operator (7.6) is shown.

With the established injectivity the inverse of the operator $I+M_{0}+i \eta N_{0} P S_{0}^{2}$ exists and is bounded from $C_{t, d}^{0, \alpha}(\partial D) \mapsto C_{t, d}^{0, \alpha}(\partial D)$ by the Riesz-Fredholm theory, i.e. the existence is proven. For the regularity results, using the Theorems 3.3 and 6.12 of [8] and the decomposition (7.7), $E^{s}$ and $H^{s}$ belong to $C^{0, \alpha}\left(\mathbb{R}^{3} \backslash D\right)$ and depend continously on the data $-2 v \times E^{i}$ in the norm of $C_{t, d}^{0, \alpha}(\partial D)$.

For the numerical examples the wave number is chosen to be no inner eigenfrequency and for simplicity in the program $\eta=0$ is used. Then the fields are given by

$$
\begin{aligned}
E^{S}(x) & =\operatorname{curl} \int_{\partial D} a(y) \Phi_{0}(x, y) \mathrm{d} s(y) \\
H^{S}(x) & =\frac{1}{i \kappa} \int_{\partial D}\left(\kappa^{2} \Phi_{0}(x, y) I+\Phi_{0}^{\prime \prime}(x, y)\right) a(y) \mathrm{d} s(y), x \in \mathbb{R}^{3} \backslash \bar{D}
\end{aligned}
$$

with $\Phi_{0}^{\prime \prime}$ being the Hessematrix of $\Phi_{0}$. This is calculated from $E^{S}(x)$ with the aid of the Maxwell equations

$$
\begin{aligned}
H^{S}(x) & =\frac{1}{i \kappa} \operatorname{curl} E^{S}(x) \\
& =\frac{1}{i \kappa} \operatorname{curl} \operatorname{curl} \int_{\partial D} a(y) \Phi_{0}(x, y) \mathrm{d} s(y)
\end{aligned}
$$


Pulling the double curl under the integral, which is only valid for $x \in \mathbb{R}^{3} \backslash \bar{D}$ due to the hypersingularity of the operator in $x=y$, using the identity

$$
\operatorname{curl} \operatorname{curl} F=-\Delta F+\operatorname{grad} \operatorname{div} F
$$

and with $a=\left(a_{1}, a_{2}, a_{3}\right)$ the magnetic field becomes

$$
\begin{aligned}
H^{s}(x)= & \frac{1}{i \kappa} \int_{\partial D} \kappa^{2} \Phi_{0}(x, y) a(y) \\
& +\operatorname{grad}\left(\partial_{1} \Phi_{0}(x, y) a_{1}(y)+\partial_{2} \Phi_{0}(x, y) a_{2}(y)+\partial_{3} \Phi_{0}(x, y) a_{3}(y)\right) \mathrm{d} s(y) \\
= & \frac{1}{i \kappa} \int_{\partial D}\left(\kappa^{2} \Phi_{0}(x, y) I+\left(\begin{array}{lll}
\partial_{1} \partial_{1} & \partial_{1} \partial_{2} & \partial_{1} \partial_{3} \\
\partial_{2} \partial_{1} & \partial_{2} \partial_{2} & \partial_{2} \partial_{3} \\
\partial_{3} \partial_{1} & \partial_{3} \partial_{2} & \partial_{3} \partial_{3}
\end{array}\right) \Phi_{0}(x, y)\right) a(y) \mathrm{d} s(y) \\
= & \frac{1}{i \kappa} \int_{\partial D}\left(\kappa^{2} \Phi_{0}(x, y) I+\Phi_{0}^{\prime \prime}(x, y)\right) a(y) \mathrm{d} s(y) .
\end{aligned}
$$

\subsection{Perfect conductor in a layered background medium}

As worked out by Akduman and Potthast [2] in the two-dimensional case it is possible to proceed analogously for a layered medium when the fundamental solution for the homogeneous case is replaced by a function solving the layered medium background problem. Thus, the ansatz for the solution theory in the two-layered medium case (LP) can be the same as the ansatz for the homogenous background medium but the fundamental solution is replaced with the Green's tensor for a two-layered medium.

Definition 7.2.1 (Direct scattering problem (LP)). With given incident fields $E_{j}^{i}, H_{j}^{i}$ which satisfy the time-harmonic Maxwell equations, find two complex-valued vector fields $E_{j}^{s}, H_{j}^{s} \in C^{1}\left(D_{j}\right) \cap C\left(\bar{D}_{j}\right), j=1,2$ such that the total fields satisfy the timeharmonic Maxwell equations

$$
\begin{aligned}
& \operatorname{curl} E_{j}(x)-i \kappa_{j} H_{j}(x)=0 \\
& \operatorname{curl} H_{j}(x)+i \kappa_{j} E_{j}(x)=0,
\end{aligned}
$$

for $x \in D_{j}, j=1,2$. The scattered field $E_{1}^{s}$ satisfy the Silver-Müller radiation condition (6.11) and the field $E_{2}^{S}$ satisfy the exponential decay (6.14) in the lower halfspace. Furthermore, the vector fields have to fulfil the transmission conditions (6.8) on the interface $S_{1}$ and the total fields the boundary conditions (6.9) on $\partial D$.

\subsubsection{Uniqueness}

Theorem 7.2.2 (Uniqueness (LP)). The scattering problem for a perfect conductor in a two-layered medium (Definition 7.2.1) has at most one solution. 
Proof. Let $E_{j}^{s, d}=E_{j}^{s, 1}-E_{j}^{s, 2}$ and $H_{j}^{s, d}=H_{j}^{s, 1}-H_{j}^{s, 2}$ for $j=1,2$ be the difference of two solutions with the same incident field $\left(E_{j}^{i}, H_{j}^{i}\right)$. Then $\left(E_{j}^{s, d}, H_{j}^{s, d}\right)$ satisfies the Maxwell's equations (6.4), the homogeneous transmission and boundary conditions (6.8 and 6.9) and the the radiation conditions (6.11) and (6.14). For simplicity, in the remainder of the proof, $E_{j}^{s, d}$ and $H_{j}^{s, d}$ will be written as $E_{j}$ and $H_{j}$.

Let $B_{R}:=\left\{x \in \mathbb{R}^{3}:|x|<R\right\}$ with radius $R>0$ a ball and denote the corresponding half-balls $B_{R, j}$ with

$$
B_{R, j}:=\left\{x \in D_{j}:|x|<R\right\}
$$

and its half-spheres

$$
\mathfrak{B}_{R, j}:=\left\{x \in D_{j}:|x|=R\right\} .
$$

Let $S_{R}$ a disk with radius $R>0$ on the interface $S_{1}$

$$
S_{R}:=\left\{x \in S_{1}:|x|<R\right\} .
$$

Further, by $v$ the outward unit normal to $\partial B_{R, j}$ and the upward unit normal to $S_{R}$ is notated. For the lower half-space, the radiation condition (6.14) implies

$$
\lim _{R \rightarrow \infty} \int_{\mathfrak{B}_{R, 2}}\left\{\left|H_{2} \times v\right|^{2}+\left|E_{2}\right|^{2}-2 \Re\left(\frac{\kappa_{1}}{\kappa_{2}} v \times E_{2} \cdot \bar{H}_{2}\right)\right\} \mathrm{d} s=0 .
$$

For sufficiently large $R$.

Using the Gauss divergence theorem in the domain $B_{R, 2} \backslash D$ leads to

$$
\int_{B_{R, 2} \backslash D} \operatorname{div}\left(E_{2} \times \bar{H}_{2}\right) \mathrm{d} x=\int_{\partial B_{R, 2}} v \times E_{2} \cdot \bar{H}_{2} \mathrm{~d} s-\int_{\partial D} v \times E_{2} \cdot \bar{H}_{2} \mathrm{~d} s .
$$

Because of the boundary condition (6.9), the integral over $\partial D$ in Equation 7.12 vanishes. Then, with the vector-identity

$$
\operatorname{div}\left(E_{2} \times \bar{H}_{2}\right)=\operatorname{curl} E_{2} \cdot \bar{H}_{2}-E_{2} \cdot \operatorname{curl} \bar{H}_{2}
$$

and from the splitting of the boundary $\partial B_{R, 2}=\mathfrak{B}_{R, 2} \cup S_{R}$ of the half-ball $B_{R, 2}$, Equation 7.12 can be written as

$$
\begin{aligned}
\int_{\mathfrak{B}_{R, 2}} v \times E_{2} \cdot \bar{H}_{2} \mathrm{~d} s= & -\int_{S_{R}} v \times E_{2} \cdot \bar{H}_{2} \mathrm{~d} s \\
& +\int_{B_{R, 2} \backslash D}\left\{\operatorname{curl} E_{2} \cdot \bar{H}_{2}-E_{2} \operatorname{curl} \bar{H}_{2}\right\} \mathrm{d} x
\end{aligned}
$$

and therefore by Maxwell equations

$$
\begin{aligned}
\int_{\mathfrak{B}_{R, 2}} v \times E_{2} \cdot \bar{H}_{2} \mathrm{~d} s= & -\int_{S_{R}} v \times E_{2} \cdot \bar{H}_{2} \mathrm{~d} s \\
& +i \int_{B_{R, 2} \backslash D}\left\{\kappa_{2}\left|H_{2}\right|^{2}-\bar{\kappa}_{2}\left|E_{2}\right|^{2}\right\} \mathrm{d} x .
\end{aligned}
$$

From this and

$$
\Re \frac{i \kappa_{1} \bar{\kappa}_{2}}{\kappa_{2}}=\Re \frac{i \kappa_{1} \bar{\kappa}_{2}^{2}}{\left|\kappa_{2}\right|^{2}}=\frac{\kappa_{1} \sigma_{2} \mu_{2} \omega}{\left|\kappa_{2}\right|^{2}}
$$


conclude that

$$
\Re \frac{\kappa_{1}}{\kappa_{2}} \int_{\mathfrak{B}_{R, 2}} v \times E_{2} \cdot \bar{H}_{2}=-\Re \frac{\kappa_{1}}{\kappa_{2}} \int_{S_{R}} v \times E_{2} \cdot \bar{H}_{2}-\frac{\kappa_{1} \sigma_{2} \mu_{2} \omega}{\left|\kappa_{2}\right|^{2}} \int_{B_{R, 2} \backslash D}\left|E_{2}\right|^{2} \mathrm{~d} x .
$$

Inserting (7.14) into (7.11) leads to

$$
\begin{aligned}
\lim _{R \rightarrow \infty}\left\{\int_{\mathfrak{B}_{R, 2}}\left\{\left|H_{2} \times v\right|^{2}+\left|E_{2}\right|^{2}\right\} \mathrm{d} s+2 \Re\right. & \frac{\kappa_{1}}{\kappa_{2}} \int_{S_{R}} v \times E_{2} \cdot \bar{H}_{2} \mathrm{~d} s \\
& \left.+\frac{2 \kappa_{1} \sigma_{2} \mu_{2} \omega}{\left|\kappa_{2}\right|^{2}} \int_{B_{R, 2} \backslash D}\left|E_{2}\right|^{2} \mathrm{~d} x\right\}=0 .
\end{aligned}
$$

For the upper half-space, first consider

$$
\int_{\mathfrak{B}_{R, 1}}\left|H_{1} \times v-E_{1}\right|^{2}
$$

Then condition (6.11) implies

$$
\begin{aligned}
& \lim _{R \rightarrow \infty} \int_{\mathfrak{B}_{R, 1}}\left\{\left|H_{1} \times v\right|^{2}+\left|E_{1}\right|^{2}-2 \Re\left(v \times E_{1} \cdot \bar{H}_{1}\right)\right\} \mathrm{d} s \\
& =\lim _{R \rightarrow \infty} \int_{\mathfrak{B}_{R, 1}}\left|H_{1} \times v-E_{1}\right|^{2} \mathrm{~d} s=0 .
\end{aligned}
$$

Again using Gauss divergence theorem and Maxwell equations following (7.12) to (7.14) yields

$$
\Re \int_{\mathfrak{B}_{R, 1}} v \times E_{1} \cdot \bar{H}_{1} \mathrm{~d} s=\Re \int_{S_{R}} v \times E_{1} \cdot \bar{H}_{1} \mathrm{~d} s
$$

and inserting (7.17) into (7.16) leads to

$$
\lim _{R \rightarrow \infty}\left\{\int_{\mathfrak{B}_{R, 1}}\left\{\left|H_{1} \times v\right|^{2}+\left|E_{1}\right|^{2}\right\} \mathrm{d} s-2 \Re \int_{S_{R}} v \times E_{1} \cdot \bar{H}_{1} \mathrm{~d} s\right\}=0 .
$$

Adding the equations (7.18) and (7.15) yields

$$
\begin{aligned}
\lim _{R \rightarrow \infty}\{ & \int_{\mathfrak{B}_{R, 1}}\left\{\left|H_{1} \times v\right|^{2}+\left|E_{1}\right|^{2}\right\} \mathrm{d} s-2 \Re \int_{S_{R}} v \times E_{1} \cdot \bar{H}_{1} \mathrm{~d} s \\
& +\int_{\mathfrak{B}_{R, 2}}\left\{\left|H_{2} \times v\right|^{2}+\left|E_{2}\right|^{2}\right\} \mathrm{d} s+2 \Re \frac{\kappa_{1}}{\kappa_{2}} \int_{S_{R}} v \times E_{2} \cdot \bar{H}_{2} \\
& \left.+\frac{2 \kappa_{1} \sigma_{2} \mu_{2} \omega}{\left|\kappa_{2}\right|^{2}} \int_{B_{R, 2} \backslash D}\left|E_{2}\right|^{2} \mathrm{~d} x\right\}=0 .
\end{aligned}
$$

From the transmission condition (6.8) it follows that

$$
v \times E_{1} \cdot \bar{H}_{1}=\frac{\kappa_{1}}{\kappa_{2}} v \times E_{2} \cdot \bar{H}_{2} \text { on } S_{1},
$$

Using this and triple scalar product identities, Equation 7.19 leads to

$$
\begin{aligned}
\lim _{R \rightarrow \infty}\left\{\int_{\mathfrak{B}_{R, 1}}\left\{\left|H_{1} \times v\right|^{2}+\left|E_{1}\right|^{2}\right\} \mathrm{d} s+\int_{\mathfrak{B}_{R, 2}}\right. & \left\{\left|H_{2} \times v\right|^{2}+\left|E_{2}\right|^{2}\right\} \mathrm{d} s \\
& \left.+\frac{2 \kappa_{1} \sigma_{2} \mu_{2} \omega}{\left|\kappa_{2}\right|^{2}} \int_{B_{R, 2} \backslash D}\left|E_{2}\right|^{2} \mathrm{~d} x\right\}=0 .
\end{aligned}
$$


By the physical setting, the constant in front of the volume integral, i.e. the third term, in Equation 7.20 is positive. Therefore, from Equation 7.20 conclude that $E_{2}=0$ in $D_{2}$ and by the first of the Maxwell equations (6.4) it also follows that $H_{2}=0$ in $D_{2}$. Then via the transmission conditions (6.8), Holmgren's theorem (see [22]) implies $E_{1}=H_{1}=0$ in $D_{1}$. Hence, $E_{j}^{s, d}=H_{j}^{s, d}=0$ in $\mathbb{R}^{3} \backslash \bar{D}$, i.e. the two solutions $E_{j}^{s, 1}$ and $E_{j}^{s, 2}$ as well as $H_{j}^{s, 1}$ and $H_{j}^{s, 2}$ are the same, which completes the proof.

\subsubsection{Existence}

The fundamental ingredient for the solution of the scattering problem is the Green's tensor (Definition 6.2.3) for the layered medium $G_{E, j}, G_{H, j}, j=1,2$.

The solution of the scattering problem is again done with a boundary integral equation approach. Seek the solution in the form of the electromagnetic field of a magnetic dipole distribution (6.59), i.e. consider the ansatz functions

$$
\begin{aligned}
E_{j}^{S}(x) & =\int_{\partial D} G_{E, j}(x, y) a(y) \mathrm{d} s(y), \\
H_{j}^{s}(x) & =\int_{\partial D} G_{H, j}(x, y) a(y) \mathrm{d} s(y),
\end{aligned}
$$

with $x \in D_{j}$, for $j=1,2$ and with a continuous tangential field $a \in C_{t, d}^{0, \alpha}(\partial D)$. Using the Maxwell equations, the relation between $E_{j}^{s}(x)$ and $H_{j}^{s}(x)$ is calculated via

$$
\begin{aligned}
H_{j}^{S}(x) & =\frac{1}{i \kappa_{j}} \operatorname{curl} E_{j}^{S}(x), \quad x \in D_{j}, \\
& =\frac{1}{i \kappa_{j}} \int_{\partial D} \operatorname{curl}_{x} G_{E, j}(x, y) a(y) \mathrm{d} s(y) \\
& =\frac{1}{i \kappa_{j}} \int_{\partial D} i \kappa_{j} G_{H, j}(x, y) a(y) \mathrm{d} s(y) \\
& =\int_{\partial D} G_{H, j}(x, y) a(y) \mathrm{d} s(y)
\end{aligned}
$$

for $j=1,2$.

Proceeding as in Section 7.1.2, i.e. using the vector Helmholtz equation (Theorem 6.1.2), the equivalence of the radiation conditions (Theorem 6.1.3) and the jump relations (Theorem 6.2.10) the fields $E^{S}, H^{S}$ defined in (7.21) solve the direct scattering problem (Definition 7.2.1) provided the density $a \in C_{t, d}^{0, \alpha}(\partial D)$ satisfies the integral equation

$$
a+M_{2} a=-2 v \times E_{2}^{i} .
$$

Remark. Here, due to the mine detection setting the wave number $\kappa_{2}$ is a complex number and as such eliminates the possibility of inner eigenfrequencies. This justifies the simpler boundary integral equation approach.

Theorem 7.2.3 (Existence (LP)). The scattering problem in Definition 7.2.1 has a unique solution. Further, the operator which maps the boundary data onto the solution is continuous from $C_{t, d}^{0, \alpha}(\partial D)$ into $C^{0, \alpha}\left(\mathbb{R}^{3} \backslash D_{j}\right) \times C^{0, \alpha}\left(\mathbb{R}^{3} \backslash D_{j}\right)$. 
Proof. To establish existence of a solution to the integral equation (7.22), by the Riesz-Fredholm theory (Theorem A.2.2) it suffices to show that $I+M_{2}$ is injective if $M_{2}$ is compact, which it is (magnetic dipole operator (Definition 6.2.11)). Then, let $a \in C_{t, d}^{0, \alpha}(\partial D)$ be a solution to the homogeneous form of (7.22). Then the fields $E_{j}^{s}, H_{j}^{s}$ given by (7.21) satisfy the homogeneous boundary condition (6.9). Therefore, by the uniqueness (Theorem 7.2.2) $E_{2}^{s}=H_{2}^{s}=0$ in $D_{2}$. From the jump relations (Theorem 6.2.10) for surface vector potentials it follows that the jumps of $E_{2}^{S}$ and $H_{2}^{s}$ when approaching $\partial D$ from inside $D$ (extending the fields inside $D$ is justified from Green's theorem (Theorem B.1.1)) are given by

$$
v \times E_{2}^{s}=-a, \quad v \times H_{2}^{s}=0 \quad \text { on } \partial D .
$$

By the Gauss' divergence theorem and the Maxwell equations following (7.12) to (7.13) and using the second equation in (7.23) leads to

$$
i \int_{D}\left\{\kappa_{2}\left|H_{2}^{s}\right|^{2}-\bar{\kappa}_{2}\left|E_{2}^{s}\right|^{2}\right\} \mathrm{d} x=\int_{\partial D} v \times E_{2}^{s} \cdot \bar{H}_{2}{ }^{s} \mathrm{~d} s=0 .
$$

From this, multiplying by $\kappa_{2}$ and taking the real part conclude that

$$
\Im\left(\kappa_{2}^{2}\right) \int_{D}\left|H_{2}^{s}\right|^{2} \mathrm{~d} x=0
$$

whence $H_{2}^{s}=0$ in $D$ follows. From this it follows via the second Maxwell equation that $E_{2}^{s}=0$ in $D$. Finally, the first equation of (7.23) implies that $a=0$, which proofs the injectivity of $I+M_{2}$ and thus the existence.

By the Riesz-Fredholm theory the inverse operator $\left(I+M_{2}\right)^{-1}$ is continuous. Therefore the regularity statement of the Theorem follows from the regularity results for surface vector potentials analogous to Theorem 7.1.3.

\subsection{Transmission problem in a homogeneous background medium}

The solution based on a boundary integral equation method for the electromagnetic transmission problem in a homogeneous background medium is analysed (See also [29] and [3]).

Definition 7.3.1 (Direct scattering problem (HT)). Given incident fields $E_{j}^{i}, H_{j}^{i}$ find two complex-valued vector fields $E_{j}^{s}, H_{j}^{s} \in \mathbb{R}^{3}, j=0,3$. Then, the incident fields, the scattered fields $E_{j}^{s}, H_{j}^{s}$ and the total field satisfy the time-harmonic Maxwell equations

$$
\begin{aligned}
& \operatorname{curl} E_{j}(x)-i \kappa_{j} H_{j}(x)=0 \\
& \operatorname{curl} H_{j}(x)+i \kappa_{j} E_{j}(x)=0
\end{aligned}
$$

for $j=0,3$ and $x \in \mathbb{R}^{3}$. Furthermore, the scattered fields have to fulfil the SilverMüller radiation condition (6.11) and the transmission conditions

$$
\begin{array}{r}
v(x) \times E_{0}^{s}(x)-b_{E} v(x) \times E_{3}^{s}(x)=f(x) \\
v(x) \times H_{0}^{s}(x)-b_{H} v(x) \times H_{3}^{s}(x)=g(x)
\end{array}
$$


on the boundary $\partial D$ of the obstacle. Assume that $f$ and $g$ are Hölder-continuous tangential fields with Hölder-continuous surface divergence on $\partial D$, i.e. $f, g \in C_{t, d}^{0, \alpha}(\partial D)$, which are given by

$$
f(x)=v \times E_{0}^{i}, \quad g(x)=v \times H_{0}^{i} .
$$

For the transmission parameters $b_{E}, b_{H} \in \mathbb{C} \backslash\{0\}$ it holds that $b_{H} \overline{b_{H}} \in \mathbb{R}^{+}$for physical reasons.

\subsubsection{Uniqueness}

Theorem 7.3.2 (Uniqueness (HT)). The scattering problem for a conducting scatterer in a homogeneous background medium (Definition 7.3.1) has at most one solution.

Proof. Let $E_{j}^{s, d}=E_{j}^{s, 1}-E_{j}^{s, 2}$ and $H_{j}^{s, d}=H_{j}^{s, 1}-H_{j}^{s, 2}$ with $j=0,3$ be the difference of two solutions with the same incident field $\left(E_{0}^{i}, H_{0}^{i}\right)$. Then $\left(E_{j}^{s, d}, H_{j}^{s, d}\right)$ satisfy the Maxwell's equations (6.4), transmission conditions (7.24) and $\left(E_{0}^{d}, H_{0}^{d}\right)$ the radiation condition (6.11). For simplicity, in the remainder of the proof, $E_{j}^{s, d}$ and $H_{j}^{s, d}$ will be written as $E_{j}$ and $H_{j}$.

The homogeneous transmission conditions (7.24) imply that

$$
\int_{\partial D} v \times E_{0} \cdot \bar{H}_{0} \mathrm{~d} s=b_{E} \bar{b}_{H} \int_{\partial D} v \times E_{3} \cdot \bar{H}_{3} \mathrm{~d} s
$$

Using the vector identity (6.17) and the Maxwell equations this becomes

$$
\int_{\partial D} v \times E_{0} \cdot \bar{H}_{0} \mathrm{~d} s=b_{E} \bar{b}_{H} i \int_{D}\left\{\kappa_{3}\left|H_{3}\right|^{2}-\bar{\kappa}_{3}\left|E_{3}\right|^{2}\right\} \mathrm{d} x
$$

Then, from $b_{E} \bar{b}_{H}=\kappa_{0} / \kappa_{3}$ and using the fact that $\kappa_{0}$ is real, the real part of the last equation is given by

$$
\Re \int_{\partial D} v \times E_{0} \cdot \bar{H}_{0} \mathrm{~d} s=-\frac{\kappa_{0} \sigma_{3} \mu_{3} \omega}{\left|\kappa_{3}\right|^{2}} \int_{D}\left|E_{3}\right|^{2} \mathrm{~d} x
$$

This implies that the assumption for the extended Rellich's Lemma (Lemma 6.1.4) is satisfied and thus applying the extended Rellich's Lemma yields $E_{0}=H_{0}=0$ in $\mathbb{R}^{3} \backslash \bar{D}$. With the transmission conditions it follows that $E_{3}=H_{3}=0$ on $\partial D$ and the Stratton-Chu formulas (Theorem B.2.1) finally imply $E_{3}=H_{3}=0$ in $D$ which completes the proof.

\subsubsection{Existence}

The transmission problem is solved with a boundary integral equation approach. Consider the ansatz functions with combined magnetic and electric dipole distribu- 
tions

$$
\begin{aligned}
E_{3}^{S}(x)= & \frac{\kappa_{3}}{\kappa_{0} b_{H}} \int_{\partial D} G_{E, 3}(x, y) \psi(y) \mathrm{d} s(y) \\
& +\frac{1}{b_{E}} \operatorname{curl} \int_{\partial D} G_{E, 3}(x, y) \varphi(y) \mathrm{d} s(y), \quad x \in D \\
E_{0}^{S}(x)= & \int_{\partial D} G_{E, 0}(x, y) \psi(y) \mathrm{d} s(y) \\
& +\operatorname{curl} \int_{\partial D} G_{E, 0}(x, y) \varphi(y) \mathrm{d} s(y), \quad x \in \mathbb{R}^{3} \backslash \bar{D}
\end{aligned}
$$

with continuous tangential fields $\varphi$ and $\psi$. The magnetic fields are given via $H_{j}(x)=$ $\frac{1}{i \kappa_{j}} \operatorname{curl} E_{j}(x)$ as

$$
\begin{aligned}
H_{3}^{s}(x)= & \frac{1}{i \kappa_{0} b_{H}} \operatorname{curl} \int_{\partial D} G_{E, 3}(x, y) \psi(y) \mathrm{d} s(y) \\
& -\frac{i \kappa_{3}}{b_{E}} \int_{\partial D} G_{E, 3}(x, y) \varphi(y) \mathrm{d} s(y), \quad x \in D \\
H_{0}^{s}(x)= & \frac{1}{i \kappa_{0}} \operatorname{curl} \int_{\partial D} G_{E, 0}(x, y) \psi(y) \mathrm{d} s(y) \\
& -i \kappa_{0} \int_{\partial D} G_{E, 0}(x, y) \varphi(y) \mathrm{d} s(y), \quad x \in \mathbb{R}^{3} \backslash \bar{D} .
\end{aligned}
$$

This ansatz functions are solutions to the transmission problem in a homogeneous medium (Definition 7.3.1) if it satisfies the transmission condition on $\partial D$. From the transmission conditions (7.24) and using the jump relations (Theorem 6.2.10) the densities has to solve the system of integral equations

$$
\begin{gathered}
\left(1+\frac{\kappa_{3} b_{E}}{\kappa_{0} b_{H}}\right) \psi+\left(M_{0}-\frac{\kappa_{3} b_{E}}{\kappa_{0} b_{H}} M_{3}\right) \psi+\left(N_{0, t}-N_{3}\right) \varphi=2 f \\
\left(1+\frac{\kappa_{3} b_{H}}{\kappa_{0} b_{E}}\right) \varphi+\left(M_{0}-\frac{\kappa_{3} b_{H}}{\kappa_{0} b_{E}} M_{3}\right) \varphi+\frac{1}{\kappa_{0}^{2}}\left(N_{0, t}-N_{3}\right) \psi=\frac{2 i}{\kappa_{0}} g
\end{gathered}
$$

with the magnetic dipole operators $M_{j}(6.54)$ and the electric dipole operator $N_{0}(6.55)$ and $N_{3}(6.58)$. For convenience the integral equations in (7.27) are summarised into the following equation

$$
\left(Y_{3}+Z_{3}\right) \chi=h
$$

where the operators $Y_{3}$ and $Z_{3}$ are given by

$$
\begin{gathered}
Y_{3}=\left(\begin{array}{cc}
1+\frac{\kappa_{3} b_{E}}{\kappa_{0} b_{H}} & 0 \\
0 & 1+\frac{\kappa_{3} b_{H}}{\kappa_{0} b_{E}}
\end{array}\right), \\
Z_{3}=\left(\begin{array}{cc}
M_{0}-\frac{\kappa_{3} b_{E}}{\kappa_{0} b_{H}} M_{3} & N_{0, t}-N_{3} \\
\frac{1}{\kappa_{0}^{2}}\left(N_{0, t}-N_{3}\right) & M_{0}-\frac{\kappa_{3} b_{H}}{\kappa_{0} b_{E}} M_{3}
\end{array}\right),
\end{gathered}
$$


the right hand side is defined by

$$
h:=2\left(\begin{array}{c}
f \\
\frac{i}{\kappa_{0}} g
\end{array}\right)
$$

and the unknown density $\chi$ has the form

$$
\chi=\left(\begin{array}{l}
\psi \\
\varphi
\end{array}\right) .
$$

Theorem 7.3.3 (Existence (HT)). The scattering problem in Definition 7.3.1 has a solution which is unique. Further, the operator which maps the boundary data onto the solution is continuous from $C_{t, d}^{0, \alpha}(\partial D)$ into $C^{0, \alpha}\left(\mathbb{R}^{3} \backslash D_{j}\right) \times C^{0, \alpha}\left(\mathbb{R}^{3} \backslash D_{j}\right)$.

Proof. The following proof follows ideas from [48] and [33]. First, examine the compactness of the boundary integral equation (7.28). Using the mapping properties of $N_{j}$ the operator $\left(N_{0, t}-N_{j}\right)$ maps $C_{t, d}^{0, \alpha}(\partial D)$ into $C_{t, d}^{0, \alpha}(\partial D)$ and is a compact operator (see Definition 6.2.12). Since also the $M_{j}$ are compact operators (see Definition 6.2.11) the operator $Z_{3}$ is a compact operator from $C_{t, d}^{0, \alpha}(\partial D)$ into $C_{t, d}^{0, \alpha}(\partial D)$. Now applying $Y^{-1}$ from the left leads to the operator $I+Y^{-1} Z_{3}$ and an equation of the second kind. $Y^{-1} Z_{3}$ is compact since $Y^{-1}$ is bounded linear and $Z_{3}$ is compact. Hence, the Riesz-Fredholm theory (Theorem A.2.2) is applicable and establishes the existence of (7.28), if the operator $\left(Y_{3}+Z_{3}\right)$ is injective. Consider the homogeneous form of (7.28). Then, $E_{j}^{s}, H_{j}^{s}$ for $j=0,3$ solve the homogeneous transmission problem and then from the uniqueness theorem it follows that $E_{j}^{s}=H_{j}^{s}=0$ in $\mathbb{R}^{3} \backslash \bar{D} \cap D$. Then the jumps on the boundary can be expressed as

$$
\begin{aligned}
v(x) \times E_{3,+}^{s}(x)-v(x) \times E_{3}^{S}(x) & =\frac{\kappa_{3}}{\kappa_{0} b_{H}} \psi(x), \\
v(x) \times E_{0}^{S}(x)-v(x) \times E_{0,-}^{s}(x) & =\psi(x), \\
v(x) \times H_{3,+}^{s}(x)-v(x) \times H_{3}^{s}(x) & =-\frac{i \kappa_{3}}{b_{E}} \varphi(x), \\
v(x) \times H_{0}^{s}(x)-v(x) \times H_{0,-}^{s}(x) & =-i \kappa_{0} \varphi(x),
\end{aligned}
$$

whereas the $E_{3,+}^{S}, H_{3,+}^{s}$ and $E_{0,-}^{S}, H_{0,-}^{s}$ are the continuations in $\mathbb{R} \backslash \bar{D}$ and in $D$ respectively. Subtracting the equations leads to the following transmission conditions

$$
\begin{gathered}
v \times E_{3,+}^{s}+\frac{\kappa_{3}}{\kappa_{0} b_{H}} v \times E_{0,-}^{s}=0, \\
v \times H_{3,+}^{s}+\frac{1}{b_{E}} v \times H_{0,-}^{s}=0 .
\end{gathered}
$$

Then $E_{3,+}^{s}, H_{3,+}^{s}$ solve the homogeneous transmission problem in $\mathbb{R}^{3} \backslash \bar{D}$ and $E_{0,-}^{s}, H_{0,-}^{s}$ solve the homogeneous transmission problem in $D$. From the uniqueness (Theorem 7.3.2) it follows that

$$
\begin{array}{ll}
E_{3,+}^{S}(x)=H_{3,+}^{s}(x)=0, & x \in \mathbb{R}^{3} \backslash \bar{D} \\
E_{0,-}^{s}(x)=H_{0,-}^{s}(x)=0, & x \in D .
\end{array}
$$


Then from (7.29) and $E_{j}^{s}=H_{j}^{s}=0$ it follows that $\psi=\varphi=0$ and thus injectivity of $\left(Y_{3}+Z_{3}\right)$ is shown.

With the established injectivity the inverse of the operator $\left(Y_{3}+Z_{3}\right)$ exists and is bounded from $C_{t, d}^{0, \alpha}(\partial D) \mapsto C_{t, d}^{0, \alpha}(\partial D)$ by the Riesz-Fredholm theory, i.e. the existence is proven.

The regularity results follows analogously as in the existence proof of the case (HP) (Theorem 7.1.3). Using the Theorems 3.3 and 6.12 of [8] and the decomposition (7.7), $E^{s}$ and $H^{s}$ belong to $C^{0, \alpha}\left(\mathbb{R}^{3} \backslash D\right)$ and depend continously on the data $-2 v \times E^{i}$ in the norm of $C_{t, d}^{0, \alpha}(\partial D)$.

\subsection{Transmission problem in a layered background medium}

The solution based on a boundary integral equation method for the electromagnetic transmission problem in a two-layered background medium is analysed.

Definition 7·4.1 (Direct scattering problem (LT)). Given incident fields $E_{j}^{i}, H_{j}^{i}$ which satisfy the time-harmonic Maxwell equations, find two complex-valued vector fields $E_{j}^{s}, H_{j}^{s} \in C^{1}\left(D_{j}\right) \cap C\left(\bar{D}_{j}\right), j=1,2,3$ such that the total fields satisfy the time-harmonic Maxwell equations

$$
\begin{aligned}
& \operatorname{curl} E_{j}(x)-i \kappa_{j} H_{j}(x)=0 \\
& \operatorname{curl} H_{j}(x)+i \kappa_{j} E_{j}(x)=0
\end{aligned}
$$

for $x \in D_{j}, j=1,2,3$. The scattered fields have to fulfil the Silver-Müller radiation condition (6.11) in the upper halfspace and the exponential decay (6.14) in the lower halfspace. Furthermore, the vector fields have to fulfil the transmission conditions (6.8) on the interface $S_{1}$ and the scattered fields the transmission condition

$$
\begin{array}{r}
v(x) \times E_{2}^{S}(x)-b_{E} v(x) \times E_{3}^{S}(x)=f(x) \\
v(x) \times H_{2}^{S}(x)-b_{H} v(x) \times H_{3}^{S}(x)=g(x)
\end{array}
$$

on the boundary $\partial D$ of the obstacle. Assume that $f$ and $g$ are Hölder-continuous tangential fields with Hölder-continuous surface divergence on $\partial D$, i.e. $f, g \in C_{t, d}^{0, \alpha}(\partial D)$. and are given by

$$
f(x)=v \times E_{1}^{i}, \quad g(x)=v \times H_{1}^{i} .
$$

For the transmission parameters $b_{E}, b_{H} \in \mathbb{C} \backslash\{0\}$ it holds that $b_{H} \overline{b_{H}} \in \mathbb{R}^{+}$due to physical reasons.

\subsubsection{Uniqueness}

Theorem 7.4.2 (Uniqueness (LT)). The scattering problem in Definition 7.4.1 for a conducting scatterer in a two-layered medium has at most one solution. 
Proof. Let $E_{j}^{s, d}=E_{j}^{s, 1}-E_{j}^{s, 2}$ and $H_{j}^{s, d}=H_{j}^{s, 1}-H_{j}^{s, 2}$ for $j=1,2,3$ be the difference of two solutions with the same incident field $\left(E_{j}^{i}, H_{j}^{i}\right)$. Then $\left(E_{j}^{d}, H_{j}^{d}\right)$ satisfies the Maxwell's equations (6.4), the transmission condition (6.8) on the interface $S_{1}$ and the homogeneous transmission condition (7.30) on the boundary of $D$. Further, $\left(E_{1}^{d}, H_{1}^{d}\right)$ satisfy the radiation condition (6.11) and $\left(E_{2}^{d}, H_{2}^{d}\right)$ the decay behaviour (6.14). For simplicity, in the remainder of the proof, $E_{j}^{s, d}$ and $H_{j}^{s, d}$ will be written as $E_{j}$ and $H_{j}$.

Let $B_{R}:=\left\{x \in \mathbb{R}^{3}:|x|<R\right\}$ with radius $R>0$ a ball and denote the corresponding half-balls $B_{R, j}$ with

$$
B_{R, j}:=\left\{x \in D_{j}:|x|<R\right\}
$$

and its half-spheres

$$
\mathfrak{B}_{R, j}:=\left\{x \in D_{j}:|x|=R\right\} .
$$

Let $S_{R}$ a disk with radius $R>0$ on the interface $S_{1}$

$$
S_{R}:=\{x \in S:|x|<R\} .
$$

Further, by $v$ the outward unit normal to $\partial B_{R, j}$ and the upward unit normal to $S_{R}$ is notated.

Then, for the lower half-space following (7.11) to (7.15) from the uniqueness proof (Theorem 7.2.2) of case (LP), using the transmission conditions instead of the boundary conditions and taking (7.26) with $\kappa_{0}$ replaced by $\kappa_{2}$ yields

$$
\begin{aligned}
\lim _{R \rightarrow \infty}\left\{\int_{\mathfrak{B}_{R, 2}}\left\{\left|H_{2} \times v\right|^{2}+\left|E_{2}\right|^{2}\right\} \mathrm{d} s+2 \Re \frac{\kappa_{1}}{\kappa_{2}} \int_{S_{R}} v \times E_{2} \cdot \bar{H}_{2} \mathrm{~d} s\right. \\
\left.\quad+\frac{2 \kappa_{1} \sigma_{2} \mu_{2} \omega}{\left|\kappa_{2}\right|^{2}} \int_{B_{R, 2} \backslash D}\left|E_{2}\right|^{2} \mathrm{~d} x+\frac{2 \kappa_{1} \sigma_{2} \mu_{2} \omega}{\left|\kappa_{2}\right|^{2}} \int_{D}\left|E_{3}\right|^{2} \mathrm{~d} x\right\}=0 .
\end{aligned}
$$

For the upper half-space again following (7.16) to (7.18) yields

$$
\lim _{R \rightarrow \infty}\left\{\int_{\mathfrak{B}_{R, 1}}\left\{\left|H_{1} \times v\right|^{2}+\left|E_{1}\right|^{2}\right\} \mathrm{d} s-2 \Re \int_{S_{R}} v \times E_{1} \cdot \bar{H}_{1} \mathrm{~d} s\right\}=0 .
$$

Analogously to uniqueness proof (Theorem 7.2.2) of case (LP) adding the equations (7.33) and (7.32), using the layer transmission condition and triple scalar product identities yields

$$
\begin{aligned}
\lim _{R \rightarrow \infty}\left\{\int_{\mathfrak{B}_{R, 1}}\left\{\left|H_{1} \times v\right|^{2}+\left|E_{1}\right|^{2}\right\} \mathrm{d} s+\int_{\mathfrak{B}_{R, 2}}\left\{\left|H_{2} \times v\right|^{2}+\left|E_{2}\right|^{2}\right\} \mathrm{d} s\right. \\
\left.\quad+\frac{2 \kappa_{1} \sigma_{2} \mu_{2} \omega}{\left|\kappa_{2}\right|^{2}} \int_{B_{R, 2} \backslash D}\left|E_{2}\right|^{2} \mathrm{~d} x+\frac{2 \kappa_{1} \sigma_{2} \mu_{2} \omega}{\left|\kappa_{2}\right|^{2}} \int_{D}\left|E_{3}\right|^{2} \mathrm{~d} x\right\}=0 .
\end{aligned}
$$

By the physical setting, the constants in front of the third and fourth term in Equation 7.34 are positive. Therefore, from Equation 7.34 conclude that $E_{2}=0$ in $D_{2}$ and $E_{3}=0$ in $D$. Then, by the first of the Maxwell equations (6.4) it also follows that $H_{2}=0$ in $D_{2}$ and via the transmission conditions (6.8) on the interface $S_{1}$, Holmgren's theorem (see [22]) implies $E_{1}=H_{1}=0$ in $D_{1}$. Hence, $E_{j}^{s, d}=H_{j}^{s, d}=0$ in $\mathbb{R}^{3} \backslash \bar{D} \cap D$, i.e. the two solutions $E_{j}^{s, 1}$ and $E_{j}^{s, 2}$ as well as $H_{j}^{s, 1}$ and $H_{j}^{s, 2}$ are the same, which completes the proof. 


\subsubsection{Existence}

The transmission problem is solved with a boundary integral equation approach. Consider the ansatz functions with a combined magnetic and electric dipole distribution

$$
\begin{aligned}
E_{3}^{S}(x) & =\frac{\kappa_{3}}{\kappa_{2} b_{H}} \int_{\partial D} G_{E, 3}(x, y) \psi(y) \mathrm{d} s(y)+\frac{1}{b_{E}} \operatorname{curl} \operatorname{curl} \int_{\partial D} \Phi_{3}(x, y) \varphi(y) \mathrm{d} s(y) \\
E_{j}^{S}(x) & =\int_{\partial D} G_{E, j}(x, y) \psi(y) \mathrm{d} s(y)+\text { curl curl } \int_{\partial D} \Phi_{j}(x, y) \varphi(y) \mathrm{d} s(y) \\
& +\int_{\partial D} \widetilde{G}_{H, j}(x, y) \varphi(y) \mathrm{d} s(y)
\end{aligned}
$$

for $x \in D_{j}, j=1,2$ with continuous tangential fields $\varphi$ and $\psi$. The magnetic fields are given through $H_{j}(x)=\frac{1}{i \kappa_{j}} \operatorname{curl} E_{j}(x)$ as

$$
\begin{aligned}
H_{3}^{s}(x) & =\frac{1}{i \kappa_{2} b_{H}} \text { curl curl } \int_{\partial D} \Phi_{3}(x, y) \psi(y) \mathrm{d} s(y)-\frac{i \kappa_{3}}{b_{E}} \int_{\partial D} G_{E, 3}(x, y) \varphi(y) \mathrm{d} s(y), \\
H_{j}^{s}(x) & =\frac{1}{i \kappa_{j}} \text { curl curl } \int_{\partial D} \Phi_{j}(x, y) \psi(y) \mathrm{d} s(y)+\int_{\partial D} \widetilde{G}_{H, j}(x, y) \psi(y) \mathrm{d} s(y) \\
& -i \kappa_{j} \int_{\partial D} G_{E, j}(x, y) \varphi(y) \mathrm{d} s(y)
\end{aligned}
$$

for $x \in D_{j}, j=1,2$. This ansatz functions are solutions to the transmission problem in a two-layered medium (Definition 7.4.1) if it satisfies the transmission condition on $\partial D$. From the transmission conditions (7.30) and using the jump relations (Theorem 6.2.10) the densities has to solve the system of integral equations

$$
\begin{gathered}
\left(1+\frac{\kappa_{3} b_{E}}{\kappa_{2} b_{H}}\right) \psi+\left(M_{2}-\frac{\kappa_{3} b_{E}}{\kappa_{2} b_{H}} M_{3}\right) \psi+\left(N_{2}-N_{3}\right) \varphi=2 f \\
\left(1+\frac{\kappa_{3} b_{H}}{\kappa_{2} b_{E}}\right) \varphi+\left(M_{2}-\frac{\kappa_{3} b_{H}}{\kappa_{2} b_{E}} M_{3}\right) \varphi+\frac{1}{\kappa_{2}^{2}}\left(N_{2}-N_{3}\right) \psi=\frac{2 i}{\kappa_{2}} g,
\end{gathered}
$$

with the magnetic dipole operators $M_{j}$ (6.54) and the electric dipole operator $N_{2}$ (6.57) and $N_{3}$ (6.58). For convenience the integral equations (7.35) are summarised into the following equation

$$
\left(Y_{4}+Z_{4}\right) \chi=h
$$

where the operators $Y_{4}$ and $Z_{4}$ are given by

$$
Y_{4}=\left(\begin{array}{cc}
1+\frac{\kappa_{3} b_{E}}{\kappa_{2} b_{H}} & 0 \\
0 & 1+\frac{\kappa_{3} b_{H}}{\kappa_{2} b_{E}}
\end{array}\right)
$$

and

$$
Z_{4}=\left(\begin{array}{cc}
M_{2}-\frac{\kappa_{3} b_{E}}{\kappa_{2} b_{H}} M_{3} & N_{2}-N_{3} \\
\frac{1}{\kappa_{2}^{2}}\left(N_{2}-N_{3}\right) & M_{2}-\frac{\kappa_{3} b_{H}}{\kappa_{2} b_{E}} M_{3}
\end{array}\right)
$$


the right hand side is defined by

$$
h:=2\left(\begin{array}{c}
f \\
\frac{i}{\kappa_{2}} g
\end{array}\right)
$$

and the unknown density $\chi$ has the form

$$
\chi=\left(\begin{array}{l}
\psi \\
\varphi
\end{array}\right) .
$$

Theorem 7.4.3 (Existence (LT)). The scattering problem in Definition 7.4.1 has a solution which is unique. Further, the operator which maps the boundary data onto the solution is continuous from $C_{t, d}^{0, \alpha}(\partial D)$ into $C^{0, \alpha}\left(\mathbb{R}^{3} \backslash D_{j}\right) \times C^{0, \alpha}\left(\mathbb{R}^{3} \backslash D_{j}\right)$.

Proof. The proof is analogous to the proof of existence for (HT) (Theorem 7.3.3). 


\section{Chapter 8}

\section{Inverse Problem}

In this chapter an inverse problem to the direct problems in Chapter 7 is defined and analysed. First, define the inverse problem under consideration.

Definition 8.o.4 (Electromagnetic inverse shape reconstruction). In the setting of Definition 6.1.1 the following data is given.

- form of the emitter and receiver loop $\Gamma_{s}, \Gamma_{r}$. They are mechanically linked such that for each measurement both the sender and receiver are moved together.

- positions of emitter and receiver, i.e. the measurement area $\mathcal{M}$.

- incident field $E^{i}$ emitted from every point in $\mathcal{M}$.

- measured induced voltages $U_{\text {meas }}$ for every point in $\mathcal{M}$.

Then, find the location and size of the buried object $D$ in $D_{2}$.

In Section 8.1 this inverse problem is reformulated as a least-squares optimisation problem. In Section 8.2 a direct search method called the Nelder-Mead algorithm is introduced which is used to solve the optimisation problem in Section 8.1. Further, the range test (Section 4.1) is expanded from the acoustic case to the electromagnetic case in Section 8.3

\subsection{An inverse optimisation problem}

The information given in the inverse problem (Definition 8.o.4) is too few information to properly reconstruct the object $D$ in a fast and robust way. Thus, to get further information find a way to characterise the earth a priori. Direct measurements of the conductivity of the background medium $\sigma_{1}, \sigma_{2}$ are not very practical. A way to do this, is to calibrate the mine detector with a scan over an area where no metallic object lies, and then use the difference of the reference scan to the actual scans. In this work, however, it is assumed that $\sigma_{1}, \sigma_{2}$ and therefore $\kappa_{1}, \kappa_{2}$ is known.

Now, describing the steps of solving the problem, begin with determining the $x_{1}$ and $x_{2}$-coordinate of the location of the obstacle. Looking at the structure of the measured data (see Figure 9.3) it is a simple task of signal analysis to determine the planar coordinates of the unknown object and its remains to find its depth and shape. 
Further, assume that the form of the unknown obstacle is approximately known beforehand. This is justified through the application of finding mines with their known metal parts. It remains to find depth and radius of the unknown obstacle. For a feasibility study in this work the obstacles are restricted to spheres and ellipses.

A simple way to achieve this, is to reformulate the problem as a least-squares optimisation problem which is then solved via a direct search method described in the next section.

Definition 8.1.1 (Optimisation problem). Given is the following data:

- the positions of the emitter and receiver, i.e. the measurement area $\mathcal{M} \subset D_{1}$,

- the form of the emitter and receiver loop $\Gamma_{s}, \Gamma_{r}$ with center $x \in \mathcal{M}$,

- the incident fields $E^{i}(x, \cdot)$ originating from $\Gamma_{s}$ for every position $x \in \mathcal{M}$,

- the measured induced voltages $U_{\text {meas }}(x)$ in the receiver loop $\Gamma_{r}$ for every position $x \in \mathcal{M}$,

- the conductivity of the background medium $\kappa_{1}, \kappa_{2}$,

- the $x_{1}$ - and $x_{2}$-coordinate of the location of the obstacle.

Let $G:=\left\{\tilde{D} \subset D_{2}\right\}$ be a set of bounded obstacles of class $C^{2}$ with connected complement and

$$
\mathcal{U}_{\text {meas }}:=\left\{U_{\text {meas }}(x): x \in \mathcal{M}\right\}
$$

the set of all measured induces voltages in $\mathcal{M}$. Denote by $F_{j}, j=\{\mathrm{HP}, \mathrm{LP}, \mathrm{HT}, \mathrm{LT}\}$ the operators for the direct problems (Definition 7.0.4) that map the incident field on the boundary $\left.E^{i}(x, \cdot)\right|_{\partial \tilde{D}}$ on to the induced voltages $U(x)$ (cf. (6.66)) and denote by

$$
\mathcal{F}_{\partial \tilde{D}}:=\left\{F_{j}\left(\left.E^{i}(x, \cdot)\right|_{\partial \tilde{D}}\right): x \in \mathcal{M}\right\}
$$

the set of calculated induced voltages for every position $x \in \mathcal{M}$ for the domain $\tilde{D}$. Then, find $\tilde{D} \subset G$ for which the functional

$$
u:=\inf _{\tilde{D} \subset G}\left\|\mathcal{F}_{\partial \tilde{D}}-\mathcal{U}_{\text {meas }}\right\|_{L^{2}}
$$

is minimised.

\subsection{Nelder-Mead simplex method}

The optimisation problem (Definition 8.1.1) can be solved using a simplex method originally developed from J. A. Nelder and R. Mead in [30]. The description of the method follows the paper of Jeffrey C. Lagarias et al. [25]. This Nelder-Mead algorithm attempts to minimise a nonlinear function mapping $\mathbb{R}^{n} \mapsto \mathbb{R}, n \in \mathbb{N}_{0}$ using only function values, i.e. without using any derivative explicitly or implicitly. The 
method is part of the class of direct search methods, which are discussed in more detail in for example [26],[49] or [45].

The basic idea of a direct search method is decribed as follows. The algorithm is based on nondegenerate simplexes which are geometric figures in $n$ dimensions of nonzero volume with $n+1$ vertices, whereas the vertices are associated with the function values. Thus, a simplex defines a triangle in $\mathbb{R}^{2}$, a tetrahedron in $\mathbb{R}^{3}$, and so on. The nondegenerate property of the simplex ensures that the set of edges adjacent to any vertex in the simplex forms a basis for the space, i.e. any point in the domain of the search can be constructed by taking linear combinations of the edges adjacent to any given vertex. In each iteration the simplex is altered through testing one or more new points which replace other points in the simplex such that the simplex fulfils some descent condition in comparison to the previous simplex. This is possible using no more than $n+1$ function values because $n+1$ vertices define a plane and $n+1$ values would be needed for a finite difference scheme. As such the simplex can be used to drive the function values towards a minimiser. A simple idea to find new points is to reflect a point through the centroid of the opposite face, which again forms a nondegenerate simplex. Then test wether the point has a better value in respect to the minimisation and if so accept the point. Doing this for all points in the simplex until a better point is found finishes an iteration and forms a new simplex. Then iterate the method until some stopping criteria is reached.

The Nelder-Mead method itself adds some other steps to increase the speed of the search but otherwise follows the main idea described above. The algorithm of the Nelder-Mead method is stated in the following.

Algorithm 5 (Nelder-Mead algorithm). Let $f(x), x \in \mathbb{R}^{n}$ be a real-valued function. Define the parameters reflection $(\rho)$, expansion $(\chi), \operatorname{contraction}(\gamma)$ and $\operatorname{shrinkage}(\sigma)$ (The notation for these parameters is only used in this section). These parameters satisfy the relations

$$
\rho>0, \quad \chi>1, \quad \chi>\rho, \quad 0<\gamma<1 \text {, and } 0<\sigma<1 \text {. }
$$

The standard parameters are chosen to be

$$
\rho=1, \quad \chi=2, \quad \gamma=\frac{1}{2}, \text { and } \sigma=\frac{1}{2}
$$

The simplex for the $k$ th iteration is denoted by $\Delta_{k}$, its vertices (points) are denoted as $x_{1}^{(k)} \ldots x_{n+1}^{(k)}$, such that

$$
f\left(x_{1}^{(k)}\right) \leq f\left(x_{2}^{(k)}\right) \leq \ldots \leq f\left(x_{n+1}^{(k)}\right) .
$$

also, for simplicity $f_{1}$ denotes $f\left(x_{1}^{(k)}\right)$. Then, $x_{1}^{(k)}$ is the best point and $x_{n+1}^{(k)}$ the worst point regarding that the goal is to minimise $f$. Further, denote by $\bar{x}=\sum_{i=1}^{n} x_{i} / n$ the centroid of the best $n$ points. Then the Nelder-Mead algorithm for the kth iteration is as follows.

1. Order. Order the $n+1$ vertices such that $f_{1}^{(k)} \leq f_{2}^{(k)} \leq \ldots \leq f_{n+1}^{(k)}$. 

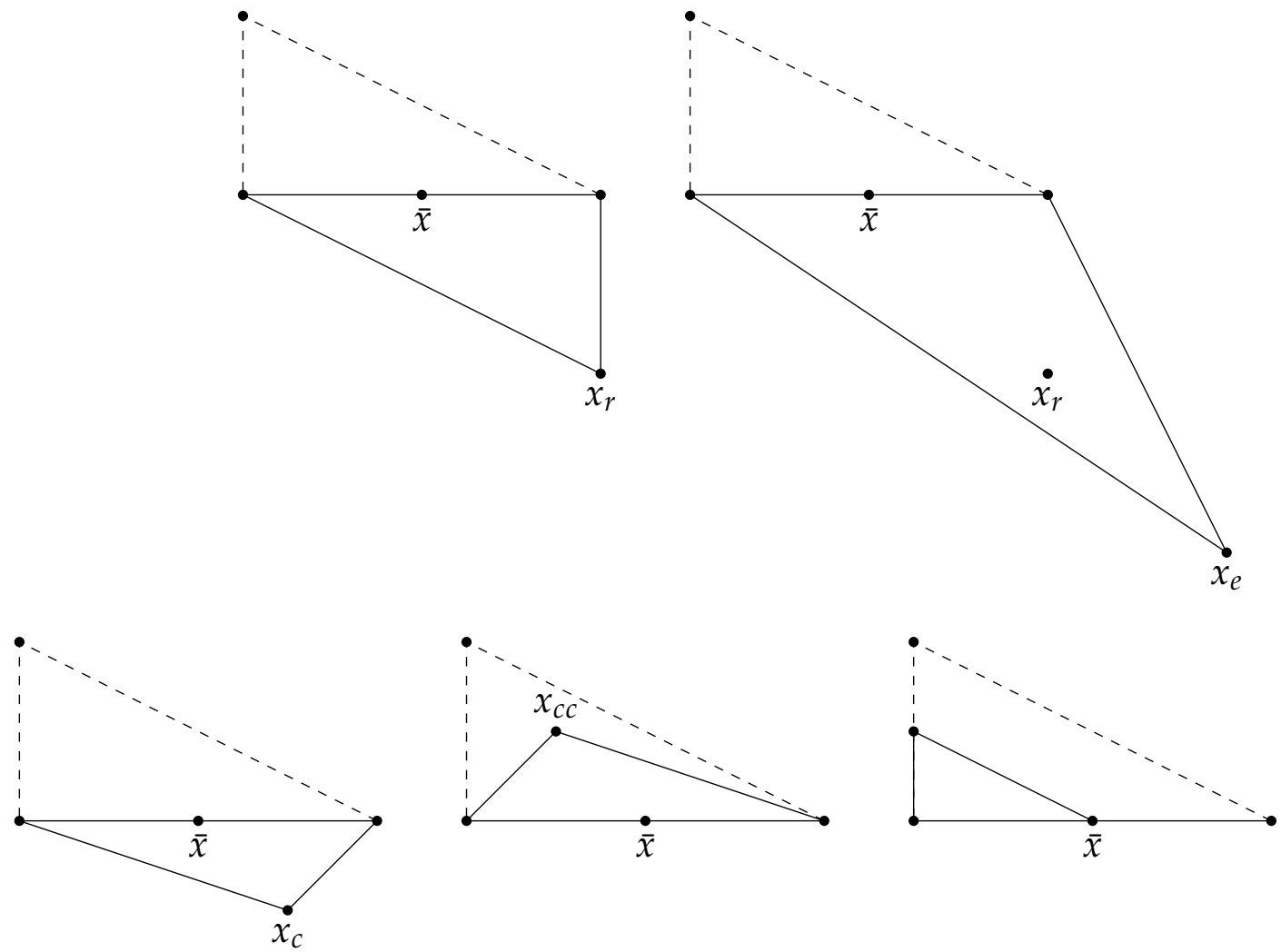

$\dot{x}_{r}$

Figure 8.1: Simplices after an iteration step. The original example simplex is shown as dashed line with the corners being the points $x_{1}, x_{2}$ and $x_{3}$. From top left to bottom right the following steps are shown: reflection, expansion, outside contraction, inside contraction and a shrink.

2. Reflect. Compute the reflection point $x_{r}$ from

$$
x_{r}=\bar{x}+\rho\left(\bar{x}-x_{n+1}\right)=(1+\rho) \bar{x}-\rho x_{n+1},
$$

and evaluate $f_{r}=f\left(x_{r}\right)$. If $f_{1} \leq f_{r}<f_{n}$, accept the reflected point $x_{r}$ and go to step $5 b$.

3. Expand. If $f_{r}<f_{1}$, calculate the expansion point $x_{e}$ via

$$
x_{e}=\bar{x}+\chi\left(x_{r}-\bar{x}\right)=\bar{x}+\rho \chi\left(\bar{x}-x_{n+1}\right)=(1+\rho \chi) \bar{x}-\rho \chi x_{n+1},
$$

and evaluate $f_{e}=f\left(x_{e}\right)$. If $f_{e}<f_{r}$, accept $x_{e}$ and if $f_{e} \geq f_{r}$ accept $x_{r}$ and go to step $5 b$.

4a. Contract (outside). If $f_{n} \leq f_{r}<f_{n+1}$, perform an outside contraction:

$$
x_{c}=\bar{x}+\gamma\left(x_{r}-\bar{x}\right)=\bar{x}+\gamma \rho\left(\bar{x}-x_{n+1}\right)=(1+\rho \gamma) \bar{x}-\rho \gamma x_{n+1},
$$

and evaluate $f_{c}=f\left(x_{c}\right)$. If $f_{c} \leq f_{r}$, accept $x_{c}$ and go to step $5 b$. 
4b. Contract (inside). If $f_{r} \geq f_{n+1}$, perform an inside contraction:

$$
x_{c c}=\bar{x}-\gamma\left(\bar{x}-x_{n+1}\right)=(1-\gamma) \bar{x}+\gamma x_{n+1},
$$

and evaluate $f_{c c}=f\left(x_{c c}\right)$. If $f_{c c}<f_{n+1}$, accept $x_{c c}$ and go to step $5 b$.

5a. Termination (shrink). Evaluate $f$ at the $n$ points $v_{i}=x_{1}+\sigma\left(x_{i}-x_{1}\right), i=$ $2, \ldots, n+1$. The vertices of the simplex at the next iteration $\Delta_{k+1}$ are $x_{1}, v_{2}, \ldots, v_{n+1}$.

5b. Termination (nonshrink). The worst point $x_{n+1}$ is discarded and the accepted point, denoted by $v^{(k)}$, is integrated at the position

$$
j=\max _{0 \leq \ell \leq n}\left\{\ell \mid f\left(v^{(k)}\right)<f\left(x_{1}^{(k)}\right)\right\} .
$$

This set of points becomes the vertices of the new simplex for the next iteration $\Delta_{k+1}$.

Remark (Properties of the Nelder-Mead algorithm). One iteration requires 1 function evaluation if the reflection point is accepted, 2 function evaluations if the expansion point or contraction point is accepted and $2 n+1$ function evaluations if a shrink is performed. The single steps of the algorithm are visualised in Figure 8.1.

\subsection{Range test}

The range test was originally developed by Roland Potthast, John Sylvester and Steven Kusiak in [41] in a acoustic setting in 2 dimensions (see also Section 4.1). Here, a variant of the range test is described for the electromagnetic case in 3 dimensions.

The inverse problem for the electromagnetic range test is given in the following definition.

Definition 8.3.1 (Electromagnetic inverse shape reconstruction). In the setting of the electromagnetic scattering (Definition 7.0.4) for a homogeneous background medium $(\mathrm{HP})$, the following data is given:

- one incident plane wave $E^{i}$ from the direction $d \in \mathbb{S}$.

- the measurement plane $M$.

- the measured scattered electric field $E^{S}$ on $M$.

Then find the shape of the unknown boundary $D$.

Remark. As with the acoustic range test, there is a simple way for the range test to improve the final reconstructed shape by using several incident waves. For every wave use the range test to get a reconstruction of the shape and then take the union of the reconstructions for a better reconstruction. 


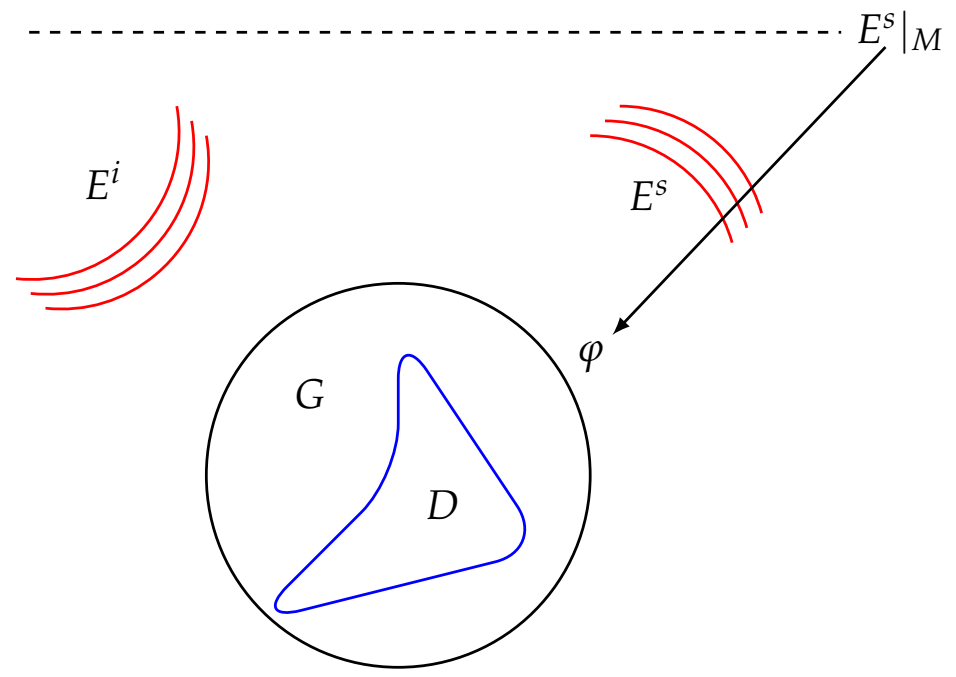

Figure 8.2: Setup and idea of the range test. Shown is one test domain $G$ containing the scatterer $D$. The idea is to get the density $\varphi$ on $G$ from the measured nearfield $\left.E^{S}\right|_{M}$ and from this the analytic extension of $E^{S}$ into $\mathbb{R}^{3} \backslash \bar{G}$ can be tested.

The basic idea of the range test in electromagnetics is to determine the maximal set on to which one scattered field may be analytically extended. Then, the complement of this set is a subset of the unknown scatterer $D$. Thus, the range test in general not deliver full reconstructions of the shape of scatterers.

Consider test domains $G$ of class $C^{2}$ with boundary $\partial G$ such that the interior Dirichlet problem for $G$ is uniquely solvable for the wave number $\kappa$ (i.e. there exist no interior eigenvalues). Then by using the operator $P_{1}$ (see 7.4) mapping from the boundary $\partial G$ onto some measurement area $M$ it can be evaluated if the scattered field $E^{S}$ is extensible into $\mathbb{R}^{3} \backslash \bar{G}$. The equation

$$
P_{1, \partial G} \varphi=\left.E^{S}\right|_{M}(\cdot, d)
$$

is solvable if $E^{S}$ can be analytically extended into $\mathbb{R}^{3} \backslash \bar{G}$ and it is not solvable if it cannot be analytically extended into $\mathbb{R}^{3} \backslash \bar{G} . P_{1, \partial G}$ is injective since when $\left.E^{S}\right|_{M}$ is 0 it follows from Holmgren's theorem that $E^{S}$ is zero everywhere. Thus, the solvability of the ill-posed integral equation (8.2) can be used as a criterion for the analytic extensibility of $E^{S}$ into $\mathbb{R}^{3} \backslash \bar{G}$. This idea and the setup for the range test is shown in Figure 8.2.

For numerical implementation of the ill-posed equation (8.2) the unbounded inverse $\left(P_{1}\right)^{-1}$ of $P_{1}$ needs to be regularised. Using the Tikhonov regularisation (Theorem A.4.3) the regularised inverse is given by

$$
R_{\alpha}:=\left(\alpha I+P_{1}^{*} P_{1}\right)^{-1} P_{1} .
$$

If the equation (8.2) is solvable, then the norm $\left\|\varphi_{\alpha}\right\|_{L^{2}}$ of

$$
\varphi_{\alpha}:=\left.R_{\alpha} E^{S}\right|_{M}(\cdot, d)
$$


will be bounded in the limit $\alpha \rightarrow 0$. If equation (8.2) does not admit a solution, then

$$
\left\|\varphi_{\alpha}\right\|_{L^{2}} \rightarrow \infty, \quad \alpha \rightarrow 0
$$

i.e. for $\alpha \rightarrow 0$ the norm of the density will tend to infinity. This is proven in Theorem $8.3 \cdot 5$.

This behaviour of the norm of the density is used to test the extensibility of the field $E^{s}$ by comparing the norm $\left\|\varphi_{\alpha}\right\|$ for solutions with a number of different test domains $G$ with some cut-off constant $C$. If for sufficiently small and fixed regularisation parameter $\alpha$ there is $\left\|\varphi_{\alpha}\right\| \leq C$, then conclude that the equation (8.2) is solvable. If in this case $\left\|\varphi_{\alpha}\right\|>C$, then conclude that it is unsolvable. In the case of solvability of (8.2) conclude that $E^{s}$ is analytically extensible into $\mathbb{R}^{3} \backslash \bar{G}$.

Now, the theory for the electromagnetic case is presented. First, remember the definition of the scattering support (Definition 4.1.2) which can be translated to the electromagnetic case by replacing the $u^{\mathcal{S}}$ with $E^{\mathcal{S}}$ and $u^{\infty}$ with $\left.E^{\mathcal{S}}\right|_{M}$.

Definition 8.3.2 (Scattering support). A domain $\Omega$ supports $\left.E^{S}\right|_{M}$ if the corresponding $E^{S}$ can be continued to solve the Helmholtz equation in $\mathbb{R}^{3} \backslash \bar{\Omega}$.

Let the incident field have the wave number $\kappa$. Then, the intersection of all convex $\Omega$ which support $\left.E^{S}\right|_{M}$ is called the convex scattering support or $c S_{\mathcal{K}}$ supp $\left.E^{S}\right|_{M}$ of the near field $\left.E^{S}\right|_{M}$

Lemma 8.3.3 (Supporting intersections). Let $\Omega_{1}$ and $\Omega_{2}$ be convex sets which support the same near field $\left.E^{S}\right|_{M}$. Then, $\Omega_{1} \cap \Omega_{2}$ supports $\left.E^{S}\right|_{M}$.

Proof. Let $E_{1}$ a continued field outside $\Omega_{1}$ and $E_{2}$ a continued field outside $\Omega_{2}$. Then Rellich's Lemma (Lemma 6.1.4) and the unique continuation Theorem for the Helmholtz equation together with the vector Helmholtz equation (Theorem 6.1.2) guarantee that the two continuations agree on the unbounded open component of $\mathbb{R}^{m} \backslash\left(\Omega_{1} \cup \Omega_{2}\right)$. If $\Omega_{1}$ and $\Omega_{2}$ are convex, then this set is connected and

$$
E_{12}= \begin{cases}E_{1} & , \text { on } \Omega_{2} \backslash\left(\Omega_{1} \cap \Omega_{2}\right) \\ E_{2} & , \text { on } \Omega_{1} \backslash\left(\Omega_{1} \cap \Omega_{2}\right) \\ E_{1} & , \text { on } \mathbb{R}^{m} \backslash\left(\Omega_{1} \cup \Omega_{2}\right)\end{cases}
$$

is well defined and satisfy the free Helmholtz equation outside $\Omega_{1} \cap \Omega_{2}$. Therefore $\Omega_{1} \cap \Omega_{2}$ supports $\left.E^{S}\right|_{M}$.

Lemma 8.3.4 (Properties of the convex scattering support). The convex scattering support has the following properties.

1. if $\left.E^{S}\right|_{M} \neq 0$ then $\left.\mathrm{CS}_{\mathcal{K}} \operatorname{supp} E^{S}\right|_{M}$ is not empty.

2. Let $E^{i}$ be the incident field illuminating a scatterer with convex hull $\Omega$ which produces the near field $\left.E^{S}\right|_{M}$. Then $\mathrm{cS}_{\mathcal{K}}$ supp $\left.E^{S}\right|_{M} \subset \bar{\Omega}$.

3. The closure of the convex scattering support $\left.\mathrm{cS}_{\mathcal{K}} \operatorname{supp} E^{S}\right|_{M}$ contains all the singularities of the scattered field $E^{s}$. 
Proof. The proof for the three properties is shown in the following.

1. If $\left.\mathrm{cS}_{\mathcal{K}} \operatorname{supp} E^{S}\right|_{M}$ is empty, then there exist at least a finite number of convex and disjunct domains $\Omega$ which support $\left.E^{S}\right|_{M}$. From Lemma 8.3.3 it follows that $E^{S}$ can be extended to a solution to the free vectorial Helmholtz equation in $\mathbb{R}^{m}$ but the only radiating solution to the free Helmholtz equation is identically zero.

2. This is a direct consequence of the Definition of the convex scattering support (Definition 8.3.2).

3. The scattered field $E^{S}$ has a unique real analytic continuation to the complement of $\left.\mathrm{cS}_{\kappa} \operatorname{supp} E^{S}\right|_{M}$, i.e. $E^{S}$ is bounded in a neighbourhood of any $x$ in that open set. This implies that unbounded neighbourhoods of any $x$ have to be in the closure of $\left.\mathrm{cS}_{\mathcal{K}} \operatorname{supp} E^{S}\right|_{M}$.

With this definition and the Lemmas the Theorem for the extensibility properties and its proof can be written up.

Theorem 8.3.5 (Extensibility properties). Assume that $\overline{\left.\mathrm{cS}_{\mathcal{K}} \operatorname{supp} E^{s}\right|_{M}} \subset$ G. Then the field $E^{s}$ can be analytically extended up to $\mathbb{R}^{m} \backslash G$ for all $x \in \mathbb{R}^{m} \backslash G$, i.e. the $L^{2}$-norms of the densities $\varphi_{\alpha}$ solving (8.3) on $\partial G$ are bounded.

If $\overline{\mathrm{cS}_{\kappa} \text { supp }\left.E^{s}\right|_{M}} \not \subset G$, then the fields $E^{s}$ cannot be extended up to $\mathbb{R}^{m} \backslash \bar{G}$ uniformly, i.e. the extensions will not be uniform in the sense that the $L^{2}$-norms of the densities $\varphi$ solving (8.3) on $\partial G$ will not be uniformly bounded.

Proof. case $\overline{\mathrm{cS}_{\kappa} \text { supp }\left.E^{S}\right|_{M}} \subset G$ : The field $E^{S}$ with nearfield $\left.E^{S}\right|_{M}$ can be analytically extended into the open exterior of the domain $G$ and into the neighbourhood of $\partial G$. The solution to the equation

$$
P_{1} \varphi=E^{S} \quad \text { on } \partial G
$$

is unique, since from [8, Theorem 6.12] and from denseness arguments $P_{1}$ maps $L^{2}(\partial G)$ bijectively into $L^{2}(\partial G)$. On the boundary $\partial G$, the vector potential $P_{1}$ now coincides with $E^{S}$ and by the solution of the exterior Dirichlet problem for the domain $G$ it coincides with $\mathbb{R}^{m} \backslash G$. Thus, the nearfield $P_{1} \varphi$ and $\left.E^{S}\right|_{M}$ coincide, i.e. $\left.E^{S}\right|_{M}$ is in the range of $\left.P_{1}^{S}\right|_{M}$. Furthermore, There exists an $\varphi \in L^{2}(\partial G)$ such that $P_{1, \partial G} \varphi=\left.E^{S}\right|_{M}$. Then, from the Tikhonov Theorem (Theorem A.4.3) the following holds

$$
\begin{aligned}
\lim _{\alpha \rightarrow 0} \varphi_{\alpha} & =\left.\lim _{\alpha \rightarrow 0}\left(\alpha I+P_{1, \partial G}^{*} P_{1, \partial G}\right)^{-1} P_{1, \partial G}^{*} E^{S}\right|_{M} \\
& =\lim _{\alpha \rightarrow 0}\left(\alpha I+P_{1, \partial G}^{*} P_{1, \partial G}\right)^{-1} P_{1, \partial G}^{*} P_{1, \partial G} \varphi \\
& =\varphi
\end{aligned}
$$

thus $\left\|\varphi_{\alpha}\right\|$ for $\alpha \rightarrow 0$ is bounded.

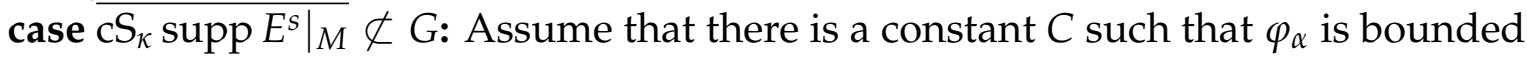
for sufficiently small $\alpha>0$. Then, there is a sequence $\alpha_{j} \rightarrow 0$ for $j \rightarrow \infty$ such that 
the weak convergence $\varphi_{\alpha_{j}} \rightarrow \tilde{\varphi}$ with some $\tilde{\varphi} \in L^{2}(\partial G)$ for $j \rightarrow \infty$ holds. The compact linear integral operator $P_{1, \partial G}: L^{2}(\partial G) \mapsto L^{2}(M)$ maps the weakly convergent sequence into a strongly convergent sequence, i.e.

$$
P_{1, \partial G} \varphi_{\alpha_{j}} \rightarrow P_{1, \partial G} \tilde{\varphi}
$$

for $j \rightarrow \infty$ and with $\left.\tilde{E}^{s}\right|_{M} \in P_{1, \partial G}\left(L^{2}(\partial G)\right)$ defined as

$$
\left.\tilde{E}^{s}\right|_{M}:=P_{1, \partial G} \tilde{\varphi} .
$$

Then passing to the limit $j \rightarrow \infty$ in

$$
\left(\alpha_{j} I+P_{1, \partial G}^{*} P_{1, \partial G}\right) \varphi_{\alpha_{j}}=\left.P_{1, \partial G}^{*} E^{S}\right|_{M}
$$

leads to

$$
P_{1, \partial G}^{*} P_{1, \partial G} \tilde{\varphi}=\left.P_{1, \partial G}^{*} E^{S}\right|_{M} \cdot
$$

From the definition of $\left.\tilde{E}^{S}\right|_{M}$ this yields

$$
\left.P_{1, \partial G}^{*} \tilde{E}^{S}\right|_{M}=\left.P_{1, \partial G}^{*} E^{S}\right|_{M},
$$

which implies $\left.\tilde{E}^{s}\right|_{M}=\left.E^{S}\right|_{M}$ from the injectivity of $P_{1, \partial G}$. Then there is a function $\varphi \in L^{2}(\partial G)$ such that $P_{1, \partial G} \varphi=\left.E^{S}\right|_{M}$. Using $P_{1}$ with density $\varphi$ the field $E^{S}$ can be analytic extended into $\mathbb{R}^{m} \backslash \bar{G}$. Thus $\bar{G}$ supports $\left.E^{S}\right|_{M}$ which leads to the contradiction $\left.\mathrm{CS}_{\mathcal{K}} \operatorname{supp} E^{S}\right|_{M} \subset \bar{G}$. This completes the proof.

The algorithm for the range test is now given analogous to the acoustic case.

Algorithm 6 (Range test). Let $\mathcal{J}$ be a finite index set and further let $\mathcal{N}:=\left\{G^{(j)}: j \in \mathcal{J}\right\}$ be the set of test domains $G^{(j)}$ which are of class $C^{2}$ such that the interior homogeneous Dirichlet problem does have only the trivial solution.

Then, use (8.3) to calculate the indicator function

$$
\mu^{(j)}:=\left\|\left.R_{\alpha} E^{S}\right|_{M}\right\|_{L^{2}(\partial G)} \cdot
$$

This is used to test whether the scattered field $E^{s}$ can be analytically extended into $\mathbb{R}^{m} \backslash \overline{G^{(j)}}$, and if so call the domain $G^{(j)}$ a positive test domain. Finally, take the intersection of all positive test domains to calculate a subset of the unknown scatterer $D$ by

$$
D_{r e c}:=\bigcap_{\mu^{(j)}<C} \bar{G}^{(j)} .
$$

Convergence for the range test is stated and proven in the following Theorem.

Theorem 8.3.6 (Convergence range test). Using sets of sampling domains $\mathcal{N}_{k}, k \in \mathcal{J}$ with index set $\mathcal{J}$, then there exist reconstructions $D_{\text {rec, },}$ with the properties $\mathrm{cS}_{\mathcal{K}}$ supp $\left.E^{S}\right|_{M} \subset$ $\bar{D}_{r e c, k}$ and $D_{r e c, k+1} \subset D_{\text {rec,k }}$ and such that for a every domain $M$ with $\left.\mathrm{cS}_{\mathcal{K}} \operatorname{supp} E^{S}\right|_{M} \subset \bar{M}$ there exists a sufficiently large $k$ such that $D_{\text {rec, } k} \subset M$. 
Proof. The reconstructions $D_{\text {rec, } k}$ with $\mathrm{cS}_{\kappa}$ supp $\left.E^{S}\right|_{M} \subset \bar{D}_{r e c, k}$ exists because of Theorem 8.3.5. The property $D_{r e c, k+1} \subset D_{r e c, k}$ is fulfilled using appropriate sampling domains in increasing number and/or better suited shapes under the condition that $\kappa$ is no Maxwell eigenvalue for the test domains (which, for example, is the case if $\Im \kappa>0$ ). For example, using spheres, with radii other than the zero points of the spherical bessel functions, it is clear that any convex domain can constructed through the intersection of sufficiently many and sufficiently big spheres. This completes the proof.

Note that as in the acoustic case the range test can not reconstruct the full shape of the obstacle using only one wave but only the convex scattering support of $D$ which is a subset of $D$. 


\section{Chapter 9}

\section{Numerics}

The goal of this chapter is the numerical realization, i.e. discretizing of the four different methods for the direct problem for electromagnetic scattering (Chapter 7) and for some simple reconstruction algorithms (Chapter 8).

\subsection{Simulation}

For the discretisation of the boundary $\partial D$ a triangulation is chosen. The $n_{S}$ triangles are denoted by $t_{l}, l=1, \ldots, n_{S}$ as

$$
t_{l}:=\left\{\boldsymbol{t}_{l}^{i} \in \partial D, i=1,2,3\right\} .
$$

Their barycentres are written as $t_{l}^{c}$ and the area of the triangle is $h_{l}$. Note that the barycentres $\boldsymbol{t}_{l}^{c}$ do not lie on the boundary $\partial D$ and thus define a approximate boundary $\partial \tilde{D}$. Further, the $\boldsymbol{t}^{c}$ can be written as a matrix with the columns corresponding to a point and each row corresponding to the coordinates of the points. Two typical triangulations are shown in Figure 9.1.

The implementation of the boundary integral operators $M_{j}, N_{j}$ in the integral equations $(7.5,7.22,7.27$ and 7.35) is done via a straightforward Nyström method (Theorem C.1.1) with a midpoint rule. Then the kernels $K(x, y)$ (i.e. the Greens tensors $\left.G_{j}(x, y)\right)$ can be written as $\boldsymbol{K}\left(\boldsymbol{t}_{l}^{c}, \boldsymbol{t}_{m}^{c}\right), l, m=1, \ldots, n_{S}$. Then, the integral operators in the generalised form

$$
F(x)=\int_{\partial D} K(x, y) a(y) \mathrm{d} s(y)
$$

are given in their discrete form as

$$
\boldsymbol{F}\left(\boldsymbol{t}_{l}^{c}\right)=\sum_{m=1, \ldots, n_{S}} \boldsymbol{K}\left(\boldsymbol{t}_{l}^{c}, \boldsymbol{t}_{m}^{c}\right) a\left(\boldsymbol{t}_{m}^{c}\right) h_{m} .
$$

The diagonal terms of the matrix $\boldsymbol{K}\left(\boldsymbol{t}_{l}^{c}, \boldsymbol{t}_{m}^{c}\right)$ are approximated through constants $c_{l}$. The case of $c_{l}=0$ is known as ignoring the singularity, which is used here. Since the integration is now over an approximate boundary $\partial \tilde{D}$ it is necessary to analyse the validity of this approximation. This is justified by the continuous dependence of the scattered field $E^{s}$ from the boundary $\partial D$, see Theorem 6.12 in [8].

For more accurate quadrature rules and handling of these integral operators with singular kernels see for example the phd-thesis of Wienert [47] or the powerful recent algorithms for scattering $[5,6,13]$. 


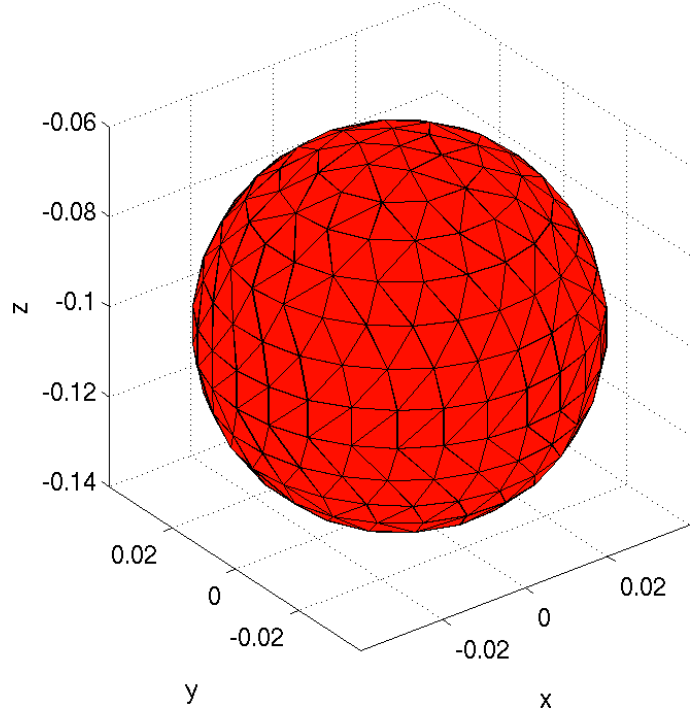

(a) sphere

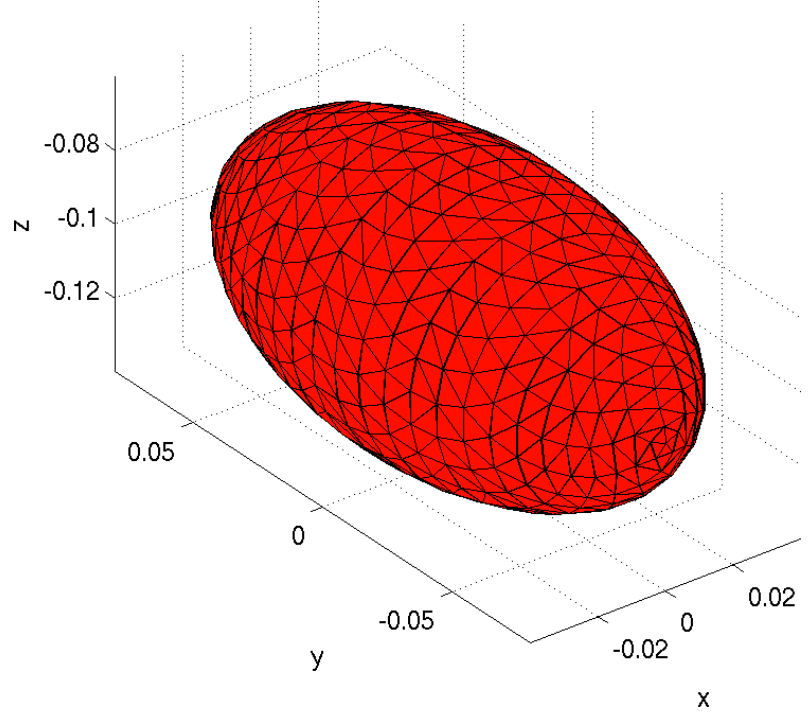

(b) ellipse

Figure 9.1: Triangulation of a sphere and ellipse which centers lies in $(0,0,-0.1) \mathrm{m}$. The sphere has a radius of $0.04 \mathrm{~m}$ and the ellipse the radii of $(0.04,0.08,0.04) \mathrm{m}$.

Here, the Green's tensors are described in their discrete and implemented form. This can be done in a general way and is valid for all tensors $G_{j}, j=0,1,2,3$. For example take the magnetic dipole operator

$$
\left(M_{j} a\right)(x)=2 v(x) \times \int_{\partial D} G_{E, j}(x, y) a(y) \mathrm{d} s(y), \quad x \in \partial D .
$$

Then the discretized form is

$$
\left(M_{j} a\right)\left(\boldsymbol{t}_{l}^{c}\right)=2 v\left(\boldsymbol{t}_{l}^{c}\right) \times \sum_{m=1, \ldots, n_{S}} G_{E, j}\left(\boldsymbol{t}_{l}^{c}, \boldsymbol{t}_{m}^{c}\right) a\left(\boldsymbol{t}_{m}^{c}\right) h_{m}
$$

for $l, m=1, \ldots, n_{S}$. For the summation the matrix $G$ is introduced. Further, for simplicity in this section, the indices $E, j$ are neglected. The matrix is constructed in such a way, that a matrix-vector multiplication yields the summation over all $\boldsymbol{t}_{m}^{c}$ for a given density $a$. For this the density $a$ is written as a vector $a$ in the following way

$$
\boldsymbol{a}=\left(a^{1}\left(\boldsymbol{t}_{1}^{c}\right), a^{2}\left(\boldsymbol{t}_{1}^{c}\right), a^{3}\left(\boldsymbol{t}_{1}^{c}\right), \ldots, a^{1}\left(\boldsymbol{t}_{n_{S}}^{c}\right), a^{2}\left(\boldsymbol{t}_{n_{S}}^{c}\right), a^{3}\left(\boldsymbol{t}_{n_{S}}^{c}\right)\right) .
$$

Then, $G$ is given by the matrix

$$
\boldsymbol{G}=\left(\begin{array}{cccccc}
\left(G e_{1}\right)^{1}\left(\boldsymbol{t}_{l}^{c}, \boldsymbol{t}_{m}^{c}\right) & \left(G e_{2}\right)^{1}\left(\boldsymbol{t}_{l}^{c}, \boldsymbol{t}_{m}^{c}\right) & \left(G e_{3}\right)^{1}\left(\boldsymbol{t}_{l}^{c}, \boldsymbol{t}_{m}^{c}\right) & \ldots & \ldots & \ldots \\
\left(G e_{1}\right)^{2}\left(\boldsymbol{t}_{l}^{c}, \boldsymbol{t}_{m}^{c}\right) & \left(G e_{2}\right)^{2}\left(\boldsymbol{t}_{l}^{c}, \boldsymbol{t}_{m}^{c}\right) & \left(G e_{3}\right)^{2}\left(\boldsymbol{t}_{l}^{c}, \boldsymbol{t}_{m}^{c}\right) & \ldots & \text { m is changing } & \ldots \\
\left(G e_{1}\right)^{3}\left(\boldsymbol{t}_{l}^{c}, \boldsymbol{t}_{m}^{c}\right) & \left(G e_{2}\right)^{3}\left(\boldsymbol{t}_{l}^{c}, \boldsymbol{t}_{m}^{c}\right) & \left(G e_{3}\right)^{3}\left(\boldsymbol{t}_{l}^{c}, \boldsymbol{t}_{m}^{c}\right) & \ldots & \ldots & \ldots \\
\vdots & \vdots & \vdots & \ddots & & \\
\vdots & 1 \text { is changing } & \vdots & & \ddots & \\
\vdots & \vdots & \vdots & & & \ddots
\end{array}\right)
$$


and the areas of the triangles are written as

$$
\boldsymbol{h}=\left(h_{1}, h_{1}, h_{1} \ldots, h_{m}, h_{m}, h_{m}\right) .
$$

For the crossproduct the vector $v$ is introduced as vectorial form of $v\left(\boldsymbol{t}_{l}^{c}\right)$, which can be written as

$$
\boldsymbol{v}=\left(v\left(\boldsymbol{t}_{1}^{c}\right), v\left(\boldsymbol{t}_{1}^{c}\right), v\left(\boldsymbol{t}_{1}^{c}\right), \ldots, v\left(\boldsymbol{t}_{n_{S}}^{c}\right), v\left(\boldsymbol{t}_{n_{S}}^{c}\right), v\left(\boldsymbol{t}_{n_{S}}^{c}\right)\right) .
$$

Then, with introducing the notation

$$
\boldsymbol{a} \odot \boldsymbol{b}:=\left(a_{1} b_{1}, \ldots, a_{n} b_{n}\right),
$$

the discretized magnetic dipole operator can be written as

$$
(M a)=2 v \times G(a \odot h) .
$$

This can be done for all boundary integral operators under consideration. Analogously, the evaluation operators $E_{j}^{s}, H_{j}^{s}$ can be handled in the same way. Then they have the form

$$
(E \boldsymbol{a})=\boldsymbol{G}(\boldsymbol{a} \odot \boldsymbol{h}) .
$$

Remark. In praxis, the implementation for the layered case, i.e. the Green's tensors $G_{j}, j=1,2$, were done in two different ways to be able to compare the complicated programs and to ensure correctness. The first version is based on the phd-thesis of Martin Petry [33] and the second implemented version is based on the theory presented in this work. One of the main differences between the two versions is that the first theoreticly handles an arbitrary number of layers, whereas the second is adopted to the problem at hand and thus uses two layers. A second major difference is the fact that the theory for the first version uses a slightly different ansatz in the layer where $x \neq y$ but which turns out to be redundant. The first one is a more complicated approach than the second. The second is therefore favoured but had some problems with the calculation of the induced voltages. Thus, both versions were used in the calculation of the numerical examples and are marked as either (I) for the first and (2) for the second.

In both versions many Hankel transforms have to be computed. In a naive way, these computations are very costly, therefore a fast method is needed. Here, the fast hankel transform (Section C.2) following [17] is used.

It is left to discretise both the integrals for the incident field from a loop (6.63) and for the induced voltage in a loop (6.66). These are straight forward discretized using rectangular quadrature rules.

For demonstration of the forward solver, the following simulation scenario is used. The setting is a simulation for the mine detection with handheld mine detectors. For the simulated mine detector the Foerster MINEX 2FD 4.500 is chosen. This handheld mine detector has a roughly circular emitter loop and a "double-D"-shaped receiver loop (see Figure 6.3). 


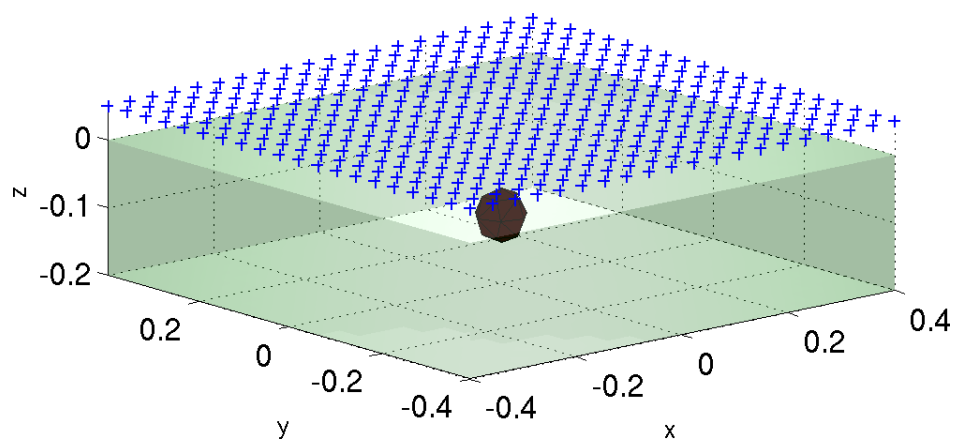

Figure 9.2: Setting for the simulation. Measurement points (blue) with the lower halfspace in transparent green and the obstacle indicated with dark red.

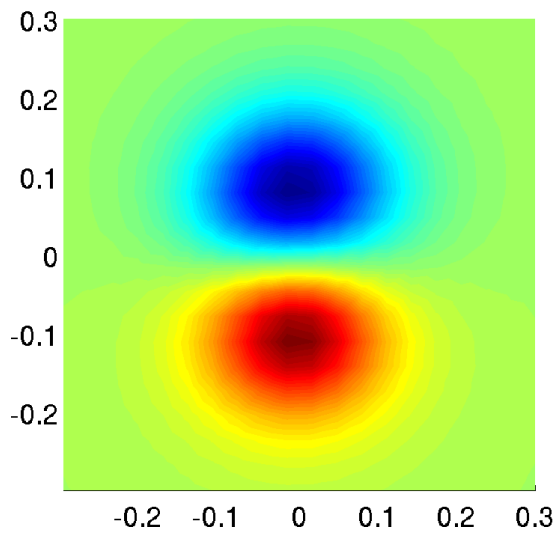

(a) sphere

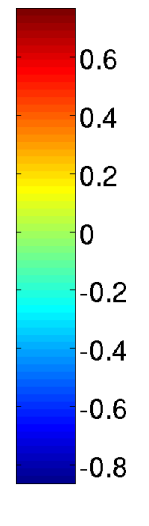

.8

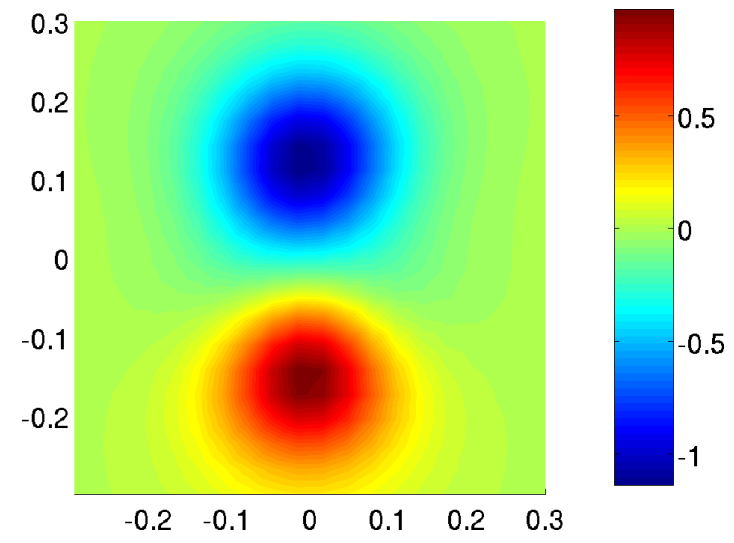

(b) ellipse

Figure 9.3: Simulated induced voltages of a homogeneous background medium with a sphere and an ellipse which lies in the position $(0,0,-0.1) \mathrm{m}$. The sphere has a radius of $0.04 \mathrm{~m}$ and the ellipse the radii of $(0.04,0.08,0.04) \mathrm{m}$.

The mine detector scans through an area $\mathcal{M}:=[-0.4,0.4]^{2} \mathrm{~m}$ in the height of $0.05 \mathrm{~m}$ which is discretized with $20 \times 20$ equidistant locations. The buried object is either a sphere of radius $0.04 \mathrm{~m}$ or an ellipsoid with semi-axis $(0.04,0.08,0.04) \mathrm{m}$ (see Figure 9.2). For every position $x \in \mathcal{M}$ the emitter sends out the incident electromagnetic field $E^{i}$, which is scattered by the buried object centered in $(0,0,-0.1) \mathrm{m}$. The scattered field $E^{S}$ from the object induces a voltage inside the receiver loop, such that the induced voltage $U$ is given by (6.66) for every point $x \in \mathcal{M}$. The resulting induced voltage is shown in the Figure 9.3.

\subsubsection{Rate of convergence}

To check the quality of the forward solver the convergence- and error rates are determined and analysed. In the case of an perfect conductor the true solution is known through theoretic argument and thus the evaluation of approximation-errors can be done. The theoretic argument is as follows: place a point source inside the obstacle. Then through the perfect conductor boundary conditions $v \times E=0$ on $\partial D$ the 


\begin{tabular}{ccccc}
\hline \multicolumn{5}{c}{ Relative error in \% } \\
$n$ & $(\mathrm{HP})$ & $(\mathrm{LP})$ & $(\mathrm{HT})$ & $(\mathrm{LT})$ \\
\hline 108 & 2.5961 & 2.5758 & 18.872 & 18.8835 \\
200 & 1.4362 & 1.4282 & 15.342 & 15.3481 \\
416 & 0.7079 & 0.70563 & 8.6864 & 8.684 \\
812 & 0.3593 & 0.36008 & 1.4082 & 1.4069 \\
1004 & 0.2918 & 0.29199 & - & - \\
1224 & 0.2479 & 0.24878 & - & - \\
\hline$O$ & 0.976 & 0.972 & 1.24 & 1.24 \\
\hline
\end{tabular}

Table 9.1: Illustration of the relative error between true and approximate induced voltage with homogenous and layered background in case (HP) and (LP), respectively and between reference and approximate induced voltage in the case (HT) and (LT). $n$ is the number of triangles on the surface of the scatterer. The columns contains the relative error in percent. The lowest line contains the rate of convergence $O$.

scattered field via the integral equation must be the same as the incident field of the point source outside of the obstacle $E_{j}^{s}(x)=E_{j}^{i}(x), x \in D_{j}, j=0,1,2$.

In the case of conducting obstacles there is no such true solution and the only way of analysing convergence rates is to use a very good discretized reference solution.

For the sake of better comparison the measurement of the error is done via comparing the induced voltages from the true or reference solution to the approximate solution. These voltages are given through a line integral over the chosen receiver loop.

Now the setting of the convergence tests is described. A "double- $\mathrm{D}$ " shaped loop is used with radius $0.15 \mathrm{~m}$ lying in a measurement plane $\mathcal{M}$ in the height $0.05 \mathrm{~m}$. The obstacle is a ball of radius $0.014 \mathrm{~m}$ centered in $z=(0,0,-0.1) \mathrm{m}$ and the incident field comes from a point source in $z=(0,0,-0.1) \mathrm{m}$. The reference solution was calculated using 916 triangles. For the two layered variants (LP) and (LT) the implementation variant (1) was used.

Conducting the tests with these settings for all cases (Definition 7.0.4) the errors and convergence rates are summed up in Table 9.1. It shows the $L^{2}$-error for several triangular meshes of the surface of the scatterer with an increasing number of triangles $n$. The rate of convergence $O$ is shown in the last line of Table 9.1.

To determine the convergence rates the error is drawn versus the number of triangles $n$ on a double-logarithmic scale in Figure 9.4. Then, through a line-fit the polynomial convergence rate is determined via the slope of the fitting line. In the cases of perfect conductor (case (HP) and (LP)) the convergence rate is of approximately of order 1 .

This observed linear convergence is expected due to a nonoptimal handling of the weak singularity for the kernel and the rough approximation of the surface of the obstacle. In the cases of a conducting obstacle (case (HT) and (LT)) the mean 
$(\mathrm{HP})$, rate $1 \pm 0.03$

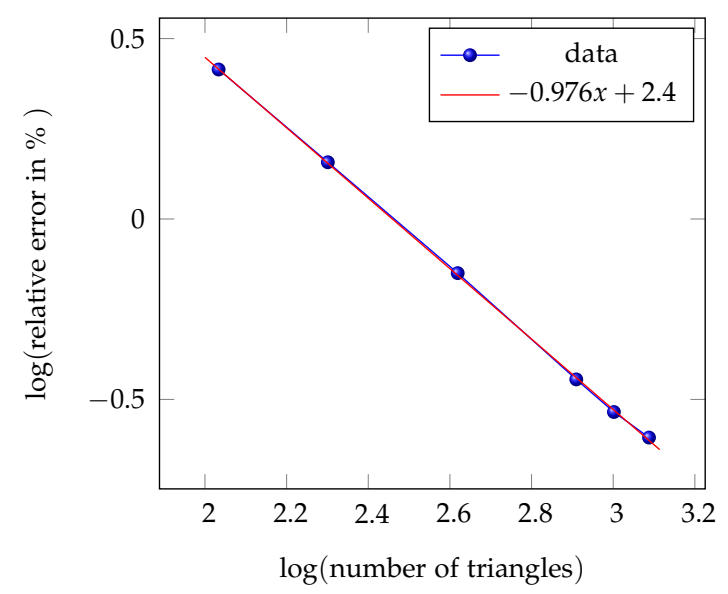

(HT), rate $1 \pm 0.25$

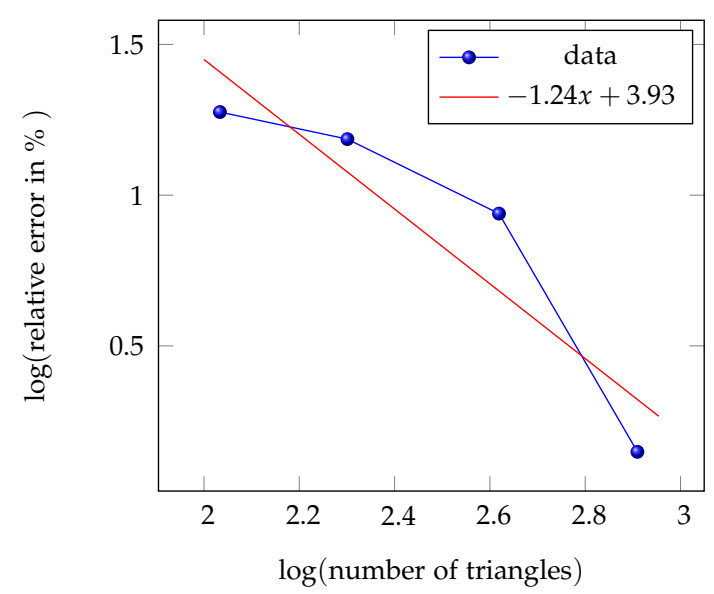

$(\mathrm{LP})$, rate $1 \pm 0.03$

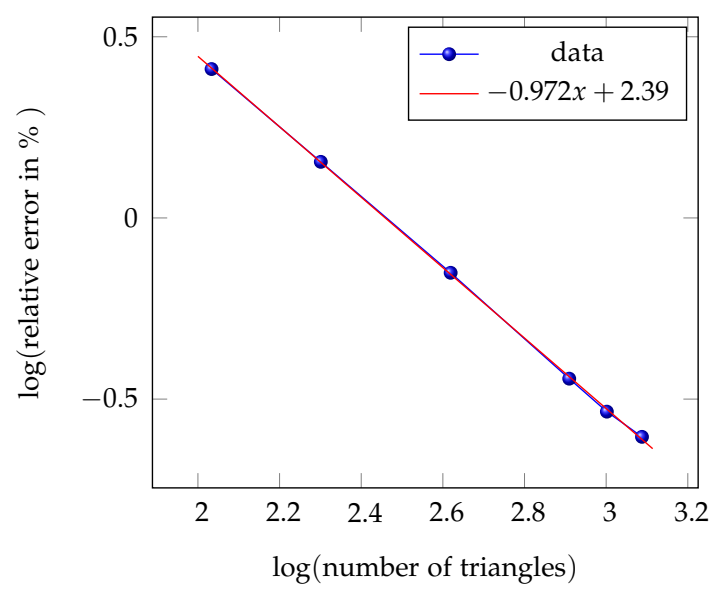

(LT), rate $1 \pm 0.25$

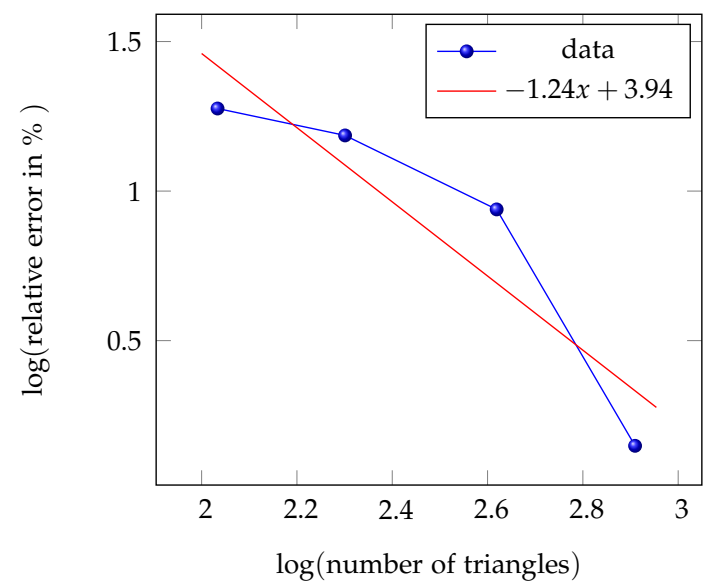

Figure 9.4: Relative error regarding number of triangles in a double logarithmic plot. The slope of the best fit approximation lines in the $L^{2}$-sense gives the approximate convergence rate of the problem under consideration.

convergence rate is of order 1.24, thus approximately linear. In theory it is also expected that the rate is of oder 1 which, in this case, is well represented through the measured order of 1.24. This is because the reference solution itself has an error and as such is reached faster.

A convergence rate of higher order is in principle possible using an appropriate handling of the singularities but is neglected here due to much higher effort for analysis and implementation.

\subsection{Nelder-Mead simplex method}

Here, some numerical examples for the reconstruction of the location of an obstacle in all four cases (Definition 7.0.4) is presented. 


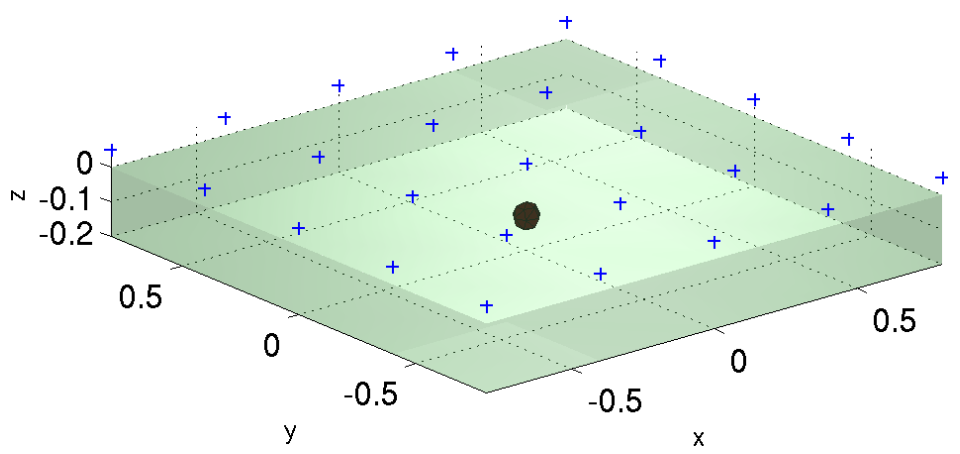

Figure 9.5: Setting for the reconstructions with the Nelder-Mead simplex method. Measurement points (blue) with the lower halfspace in transparent green and the obstacle indicated with dark red.

The given optimisation problem (Definition 8.1.1) is handled with the Nelder-Mead simplex method. The implementation of the method is taken from an external source and is directly applied to (8.1). The simulated data for this reconstructions are calculated with the following setting. Again, a circular emitter loop and a "double-D"shaped receiver loop is used (Figure 6.3) which are moved together as a simulated mine detector. The mine detector scans through an area $\mathcal{M}:=[-0.8,0.8]^{2} \mathrm{~m}$ in the height $0.05 \mathrm{~m}$ which is discretized with $5 \times 5$ equidistant locations of the detector. The buried object is either a sphere of radius $0.04 \mathrm{~m}$ or an ellipsoid with semi-axis $(0.04,0.08,0.04) \mathrm{m}$, both centered in $(0,0,-0.1) \mathrm{m}$. No noise is applied to the data. The setting is shown in Figure 9.5.

In the following the reconstructions of size, location with additionally known orientation of the obstacle are shown in the following Figures 9.6, 9.8, 9.7 and 9.9 corresponding to the four cases (Definition 7.0.4). Each single image shows the state of the reconstruction to a given iteration of the algorithm. The true obstacle has a transparent blue colour and the reconstructed obstacle is painted in transparent red. Every Figure shows reconstructions for spheres and ellipses.

Case (HP) is the case of homogeneous background medium with a perfect conductor obstacle and is shown in Figure 9.6.

Case (LP) is the case of layered background medium with a perfect conductor obstacle and is shown in Figure 9.7. The implementation variant (2) is used here.

Case (HT) is the case of homogenous background with a conducting obstacle and is shown in Figure 9.8.

Case (LT) is the case of layered background medium with a conducting obstacle and is shown in Figure 9.9. The implementation variant (2) is used here.

In all cases the reconstruction of the true obstacle was successful. The examples exhibit the feasibility of the proposed method to identify the depth and size parameters of a buried metallic object via scattering theory and a direct search method. 


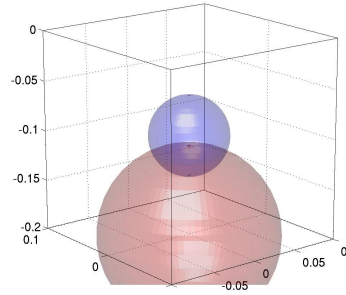

(a) Iteration 5

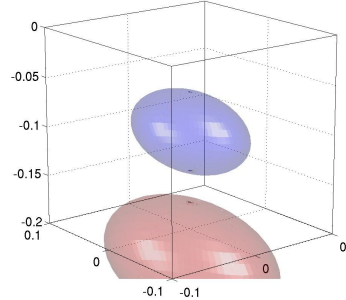

(e) Iteration 5

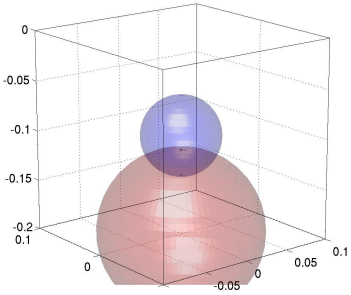

(b) Iteration 10

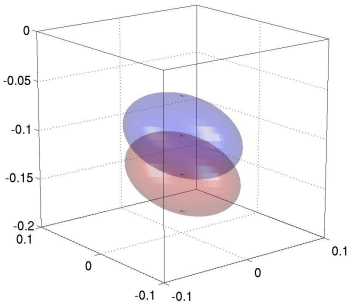

(f) Iteration 15

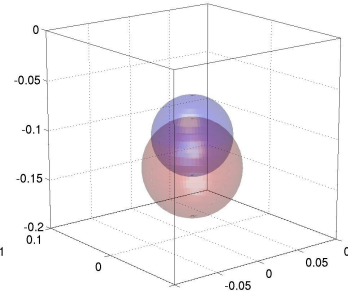

(c) Iteration 15

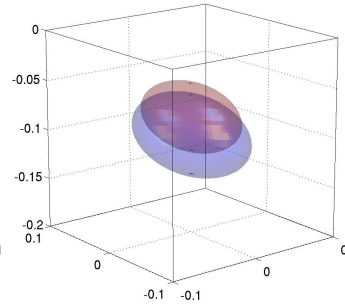

(g) Iteration 20

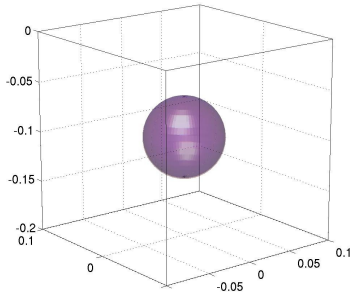

(d) Iteration 20

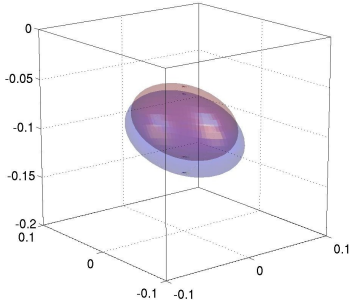

(h) Iteration 25

Figure 9.6: Shape reconstructions in a homogenous background with a perfect conductor (case (HP)). The top line shows iterations of the reconstruction of a sphere. The bottom line shows iterations for an ellipse. Scatterer (blue), Reconstruction (red).

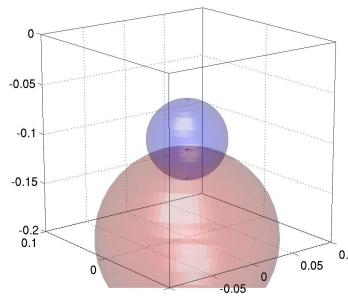

(a) Iteration 5

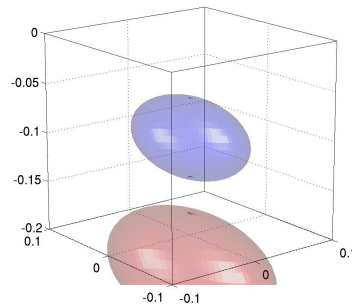

(e) Iteration 10

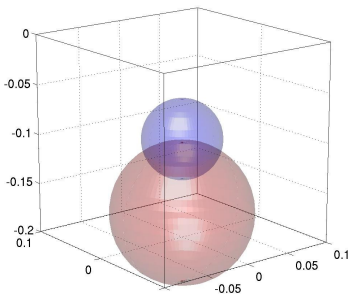

(b) Iteration 15

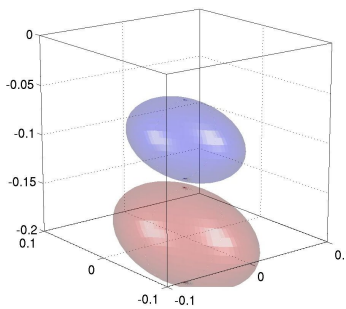

(f) Iteration 15

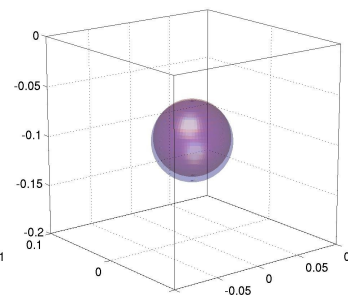

(c) Iteration 25

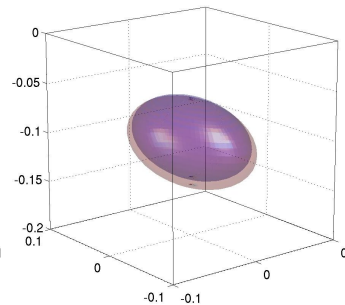

(g) Iteration 20

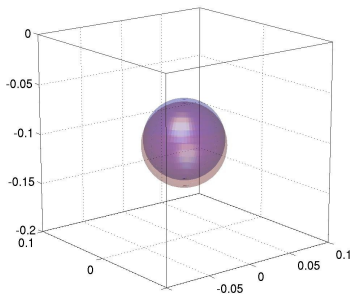

(d) Iteration 30

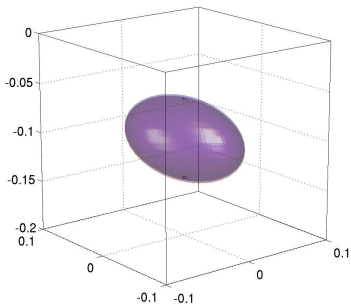

(h) Iteration 25

Figure 9.7: Shape reconstructions in a layered background with a perfect conductor (case (LP)). The top line shows iterations of the reconstruction of a sphere. The bottom line shows iterations for an ellipse. Scatterer (blue), Reconstruction (red). Implementation variant (2). 


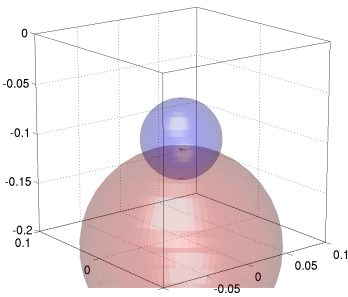

(a) Iteration 5

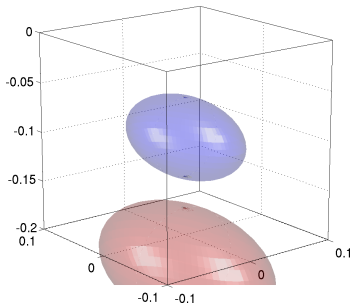

(e) Iteration 5

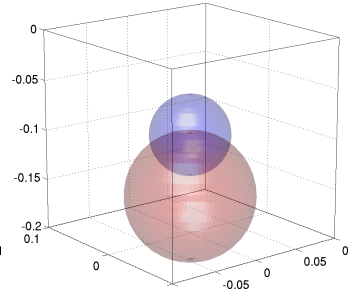

(b) Iteration 15

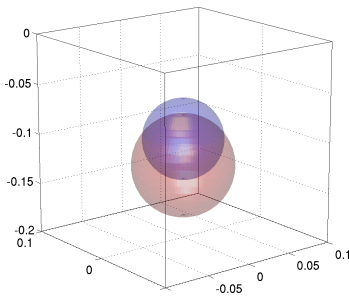

(c) Iteration 20

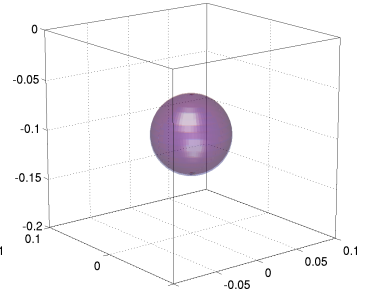

(d) Iteration 30

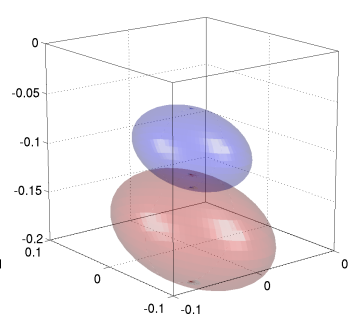

(f) Iteration 15

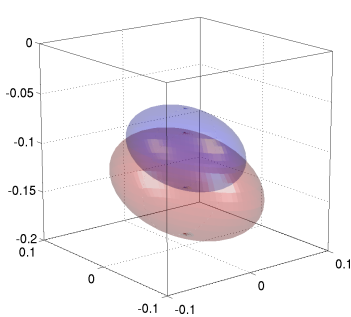

(g) Iteration 20

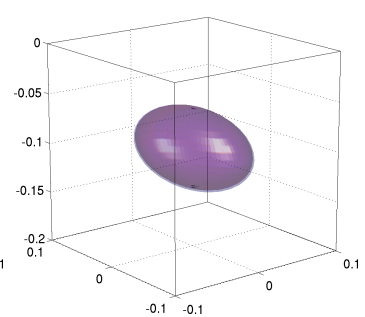

(h) Iteration 30

Figure 9.8: Shape reconstructions in a homogenous background with a conducting obstacle (case (HT)). The top line shows iterations of the reconstruction of a sphere. The bottom line shows iterations for an ellipse. Scatterer (blue), Reconstruction (red).

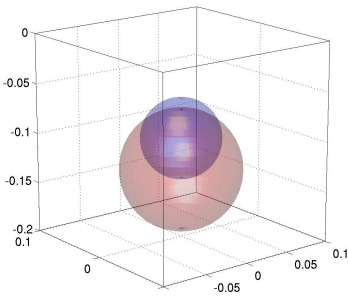

(a) Iteration 25

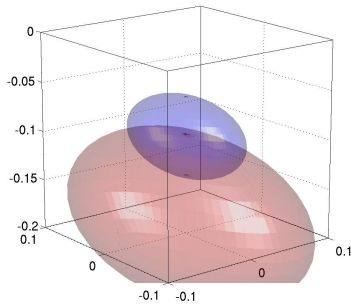

(e) Iteration 10

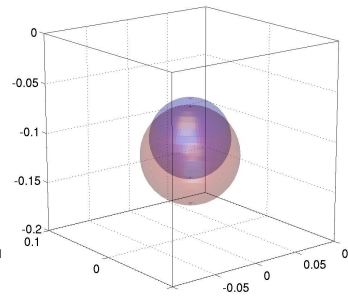

(b) Iteration 30

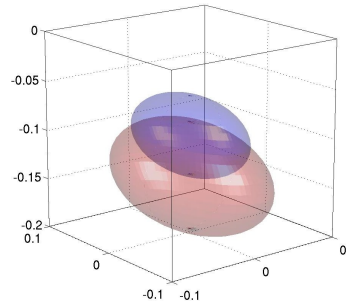

(f) Iteration 15

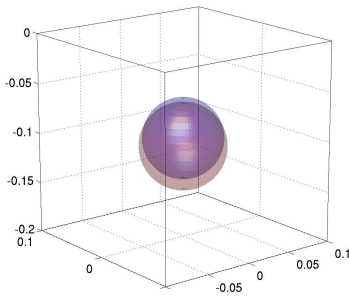

(c) Iteration 35

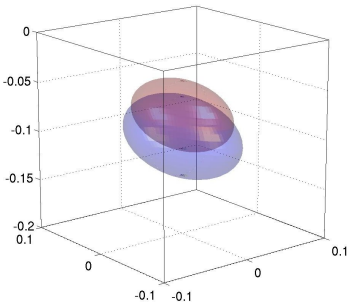

(g) Iteration 20

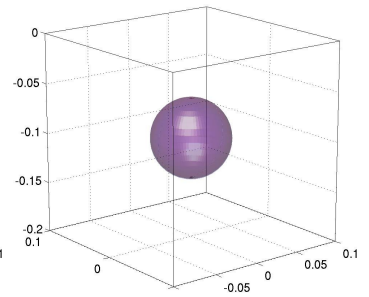

(d) Iteration 50

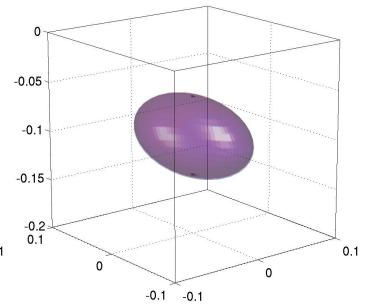

(h) Iteration 25

Figure 9.9: Shape reconstructions in a layered background medium with a conducting obstacle (case (LT)). The top line shows iterations of the reconstruction of a sphere. The bottom line shows iterations for an ellipse. Scatterer (blue), Reconstruction (red). Implementation variant (2). 


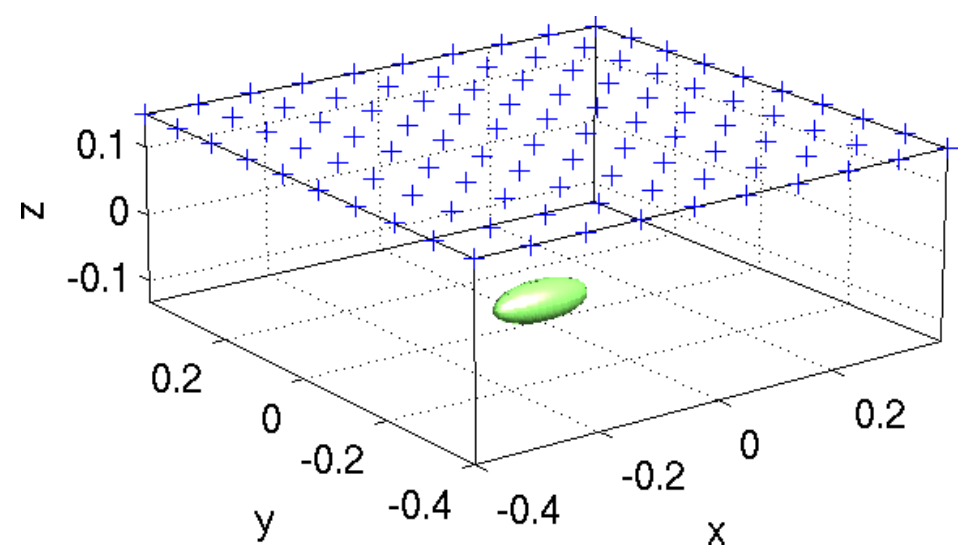

Figure 9.10: Setting for the reconstructions with the range test. Measurement points (blue) with the obstacle indicated in green. One incident plane wave from above.

\subsection{Range test}

Here, numerical implementation and examples for the range test for one incident plane wave in the electromagnetic setting is presented. The setting with homogeneous background and a perfect conductor obstacle (HP) is used here, since for a first step with the range test in electromagnetics the restriction to a simple and fast computable setting is preferable to minimise errors.

Since here, like in acoustic, plane waves are used they are introduced in the electromagnetic case. Note, that the range test is not depending on the type of incoming field. A plane wave was chosen for better comparison to the range test in the acoustic case.

Definition 9.3.1 (Electromagnetic plane wave). In the setting of time-harmonic Maxwell equations a electromagnetic plane wave with direction of propagation $d$ in $\mathbb{R}^{3}$ with wave number $\kappa_{j}(6.5)$ is given by

$$
E_{j}^{i}(x)=p e^{i\left(\kappa_{j} x \cdot d\right)} .
$$

As described in theory of the range test (Section 8.3), the exact shape of the scatterer cannot be reconstructed. However, it is still possible to obtain some reconstructions which reflects basic properties of the shape of the scatterer under consideration.

Analogous to the derivation of (9.3) the evaluation operator $P_{1}$ for the test domain $G^{(j)}$ can be written in his discretized form as

$$
\left(\boldsymbol{P}_{1}^{(j)} \boldsymbol{a}\right)=\boldsymbol{G}(\boldsymbol{a} \odot \boldsymbol{h})
$$

For the stabilised inversion of $\boldsymbol{P}_{1}$ again the Tikhonov regularisation (Theorem A.4.3) is used, i.e. calculate

$$
\boldsymbol{R}_{\alpha}^{(j)}:=\left(\alpha \boldsymbol{I}+\boldsymbol{P}_{1}^{(j), *} \boldsymbol{P}_{1}^{(j)}\right)^{-1} \boldsymbol{P}_{1}^{(j), *}
$$



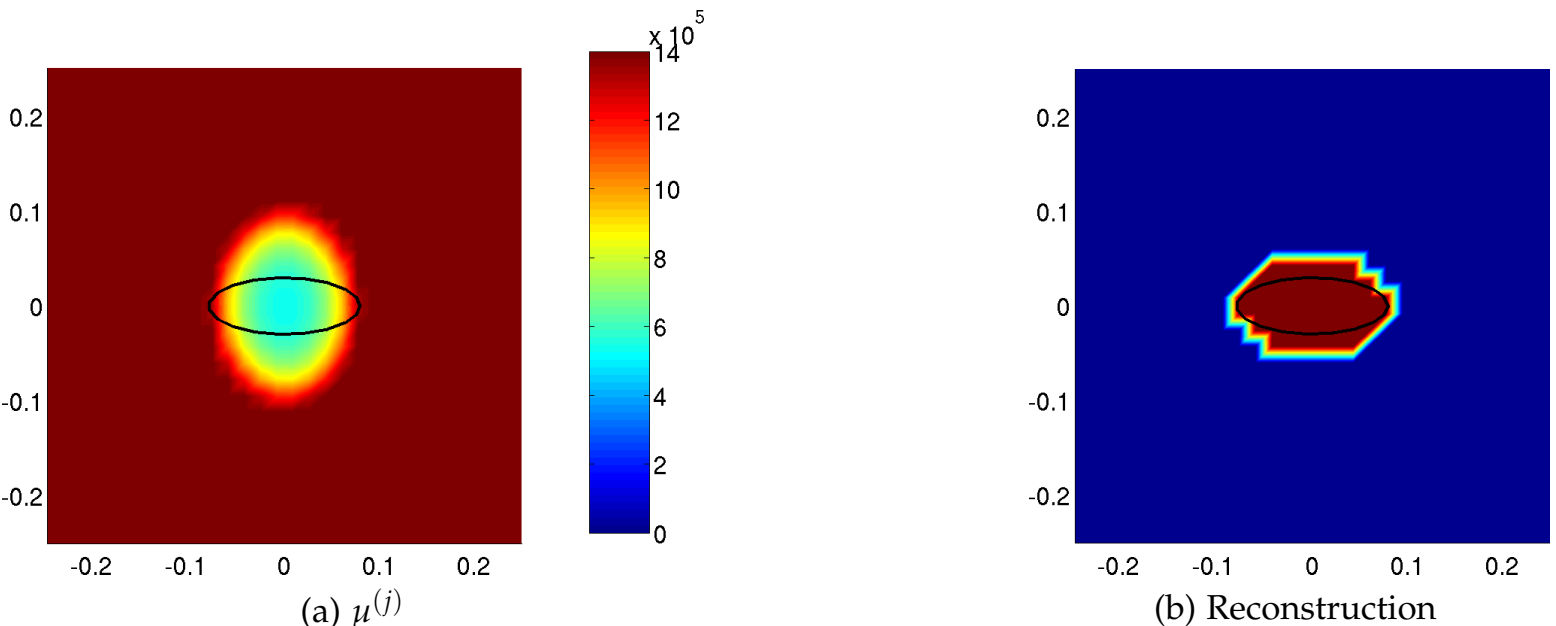

(b) Reconstruction

Figure 9.11: Reconstructions with the range test for an ellipse in a slice horizontally through the center of the obstacle. The true object is indicated through a black circle. (a) shows the norm of the density where the colours are red if the norm is above the cutoff and (b) shows the reconstruction.

for $\alpha>0$. Here $*$ denotes the complex conjugate transposed matrix and $I$ is the identity matrix.

Then for all $j \in \mathcal{J}$, the density living on the boundary of the test domains is calculated via

$$
\boldsymbol{\varphi}^{(j)}:=\left.\boldsymbol{R}_{\alpha}^{(j)} \circ \boldsymbol{E}^{S}\right|_{M}(d)
$$

for all test domains $G^{(j)}$ and for the nearfield $\left.\boldsymbol{E}^{S}\right|_{M}(d)$. Then the functional $\mu^{(j)}(8.5)$ can be written as

$$
\boldsymbol{\mu}^{(j)}=\sqrt{\boldsymbol{\varphi}^{(j), T} \cdot \boldsymbol{\varphi}^{(j)}} .
$$

The size of the real number $\mu^{(j)}$ is an indicator for the extensibility of the field $\left.\boldsymbol{E}^{S}\right|_{M}(d)$ into the exterior of the test domain $G^{(j)}$.

Numericly, a cut-off parameter is needed to decide whether the test domain is positive or negative. The cut-off parameter $C$ can be determined by calibration: use a simulated nearfield for a given domain, carry out the reconstruction and choose $C$ appropriately to achieve reasonable reconstructions for the domain under consideration. Then, use the same constant $C$ for other domains as well.

For the reconstruction examples the following parameters and settings were used. The measurement area is given by $\mathcal{M}:=[-0.4,0.4]^{2} \mathrm{~m}$ in the height of $0.25 \mathrm{~m}$ which is discretized with $10 \times 10$ equidistant points. The regularisation parameter $\alpha$ is kept fixed with the value $\alpha=10^{-9}$. For simplicity spheres of radius $r=0.15 \mathrm{~m}$ and 252 triangles are used as test domains $G$ and the search grid is constructed by $M_{s}=[-0.25,0.25] \times[-0.25,0.25] \times[-0.35,0.15] \mathrm{m}$ such that every $x_{j} \in M_{s}$ is the center of the corresponding test domain $G^{(j)}$. The one incoming plane wave has the direction $(0,0,-1)$. The setup is shown in Figure 9.10.

In the first example the obstacle is an ellipse with radii $r=(0.08,0.03,0.03) \mathrm{m}$ with the center lying in $(0,0,-0.1) \mathrm{m}$. The results are shown in Figure 9.11 and Figure 9.12. 


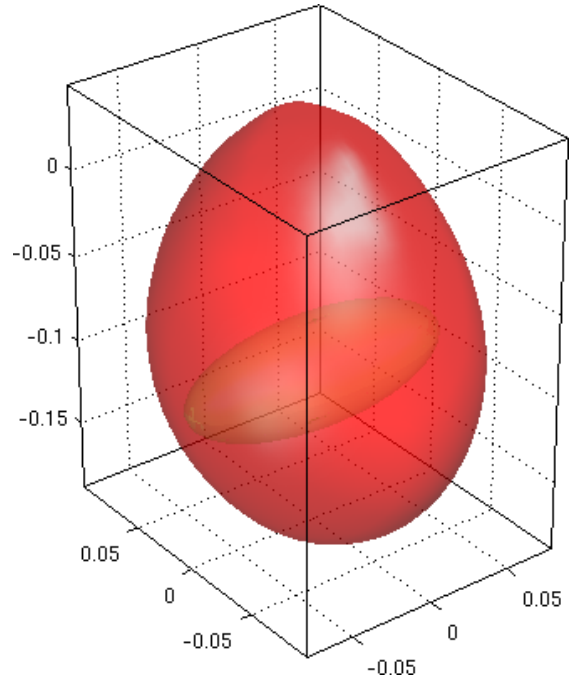

(a) surface $\mu^{(j)}=C$

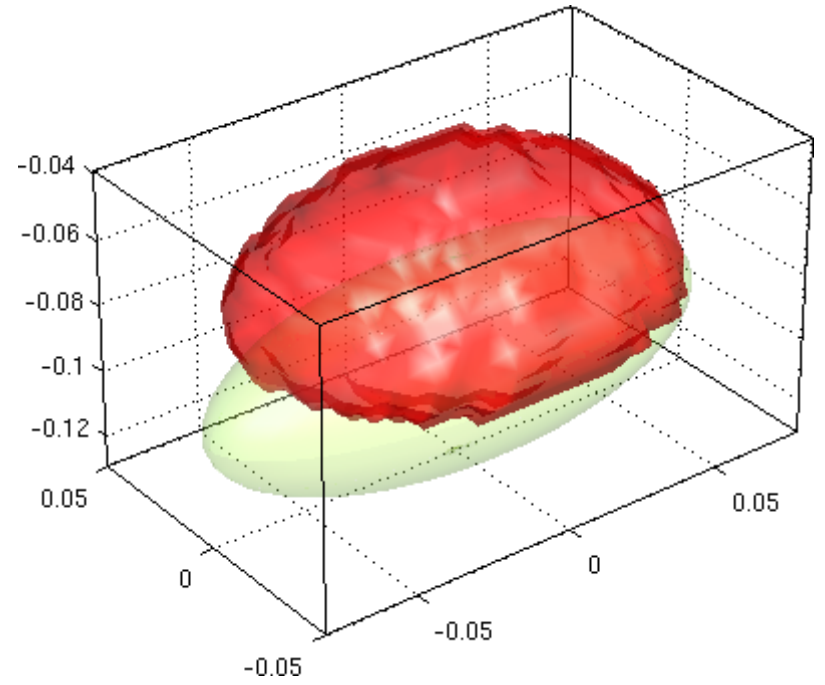

(b) Reconstruction

Figure 9.12: Reconstructions with the range test for an ellipse. The true object is indicated through a transparent green surface. (a) shows the surface of the cut in the density and (b) shows the reconstruction as a transparent red surface in $3 \mathrm{D}$.

In the second example the obstacle is a sphere with radius $r=0.04 \mathrm{~m}$ with the center lying in $(0,0,-0.1) \mathrm{m}$. The results are shown in Figure 9.13 and Figure 9.14. Both figures contain four images each. The subfigures a) and b) show images for a horizontal slice through the center of the obstacle where the slice of the surface of the true object is represented as a black curve. The subfigures c) and d) show images regarding a cubic search grid where the surface of the true object is shown in transparent green. In a) the functional $\mu^{(j)}$ is shown. The cut-off parameter $C$ is incorporated such that the colour is dark red (top of colourscale) when $\mu^{(j)}>C$. b) then shows the reconstruction of the unknown obstacle in dark red colour. In c) the surface with $\mu^{(j)}=C$ is shown in transparent red. At last in d) the reconstruction of the unknown obstacle is presented in a red surface.

The reconstructions in the slices seem to be much better than in the full ${ }_{3} \mathrm{D}$-case. This is mainly because the calculated $\mu^{(j)}$ becomes more blurred along the z-axis, which can be seen from the "egg"-like surfaces in the plots of $\mu^{(j)}$. This seems to be an effect of the small measurement area $M$ above the obstacle. Due to the intersection of the corresponding test domains this then leads to an cutoff of the lower parts of the reconstruction, which leaves the impression of getting only reconstructions which are lying to high. This problem doesn't occur in the slice-reconstructions such that this reconstructions can be quite good, dependent of height. Without knowing the boundary condition, from one incoming wave and with this small measurement area the ${ }_{3} \mathrm{D}$ reconstruction is reasonable good to find objects and their size which would be the main task in the application of mine detection. 


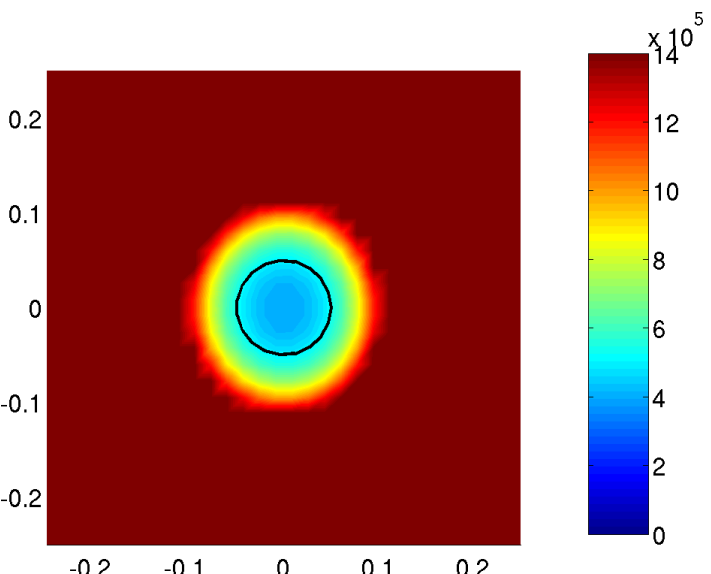

(a) $\mu^{(j)}$

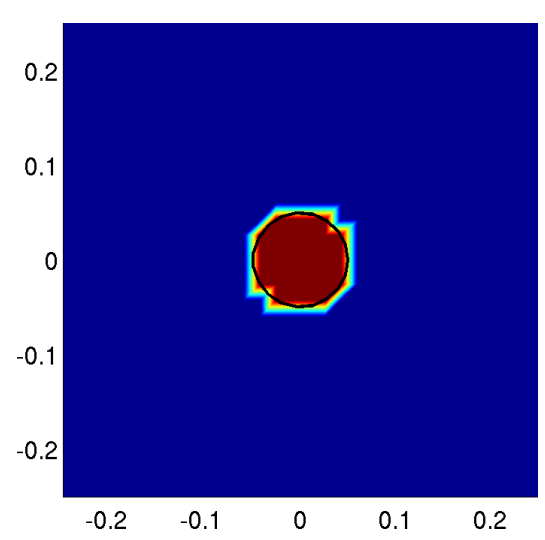

(b) Reconstruction

Figure 9.13: Reconstructions with the range test for a sphere in a slice horizontally through the center of the obstacle. The true object is indicated through a black circle. (a) shows the norm of the density where the colours are red if the norm is above the cutoff and (b) shows the reconstruction.

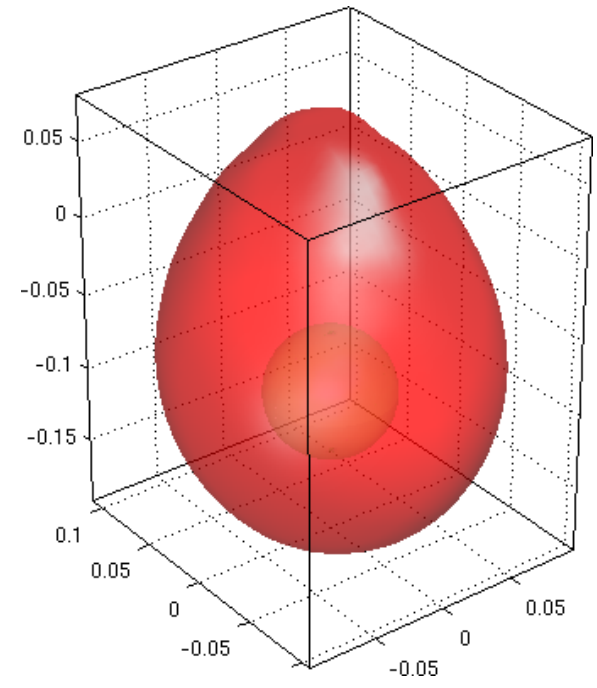

(a) surface $\mu^{(j)}=C$

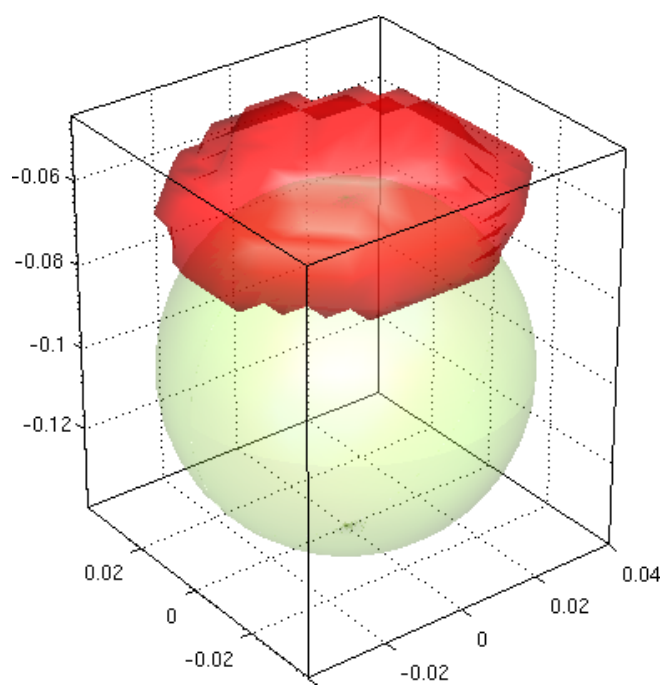

(b) Reconstruction

Figure 9.14: Reconstructions with the range test for a sphere. The true object is indicated through a transparent green surface. (a) shows the surface of the cut in the density and (b) shows the reconstruction as a transparent red surface in $3 \mathrm{D}$. 
Chapter 9 Numerics 
Part III

Appendix 



\section{Appendix A}

\section{Integral equations}

The chapter is organised as follows. Compact integral operators are introduced in Section A.1. Following Frigyes Riesz and Erik Ivar Fredholm who developed a theory for solving integral equations of the second kind with compact operators which is described in Section A.2. Then, Section A.3 covers the concept of ill-posed equations which leads to numerical instabilities. These can be overcome with so called regularisation schemes, presented in Section A.4.

\section{A.1 Operators}

Compact integral operators are playing an important role in this work and some of their properties are briefly summed up in this section.

Definition A.1.1 (Compact operators). Let $X, Y$ be normed spaces and $A: X \mapsto Y$ a linear operator, then it is called compact if it maps each bounded set in $X$ in a relatively compact set in $Y$.

Remark (Properties of compact operators). Compact operators have the following properties:

- They are bounded.

- Linear combinations are again compact.

- They have no continuous inverse.

An important class of compact operators is given by integral operators with continuous or weakly singular kernels.

Definition A.1.2 (weakly singular, domain). A continuous kernel $K(x, y), x, y \in D \subset$ $\mathbb{R}^{m}, x \neq y$ is called weakly singular if there exists positive constants $C$ and $a \in(0, m]$ such that

$$
|K(x, y)| \leq C|x-y|^{a-m}, \quad x, y \in D, x \neq y
$$


Now, briefly describe integral operators for the application in integral equations and the particular problems in scattering theory.

Theorem A.1.3 (Integral operator, domain). Let $D \subset \mathbb{R}^{3}$ be a nonempty compact and Jordan measurable set that coincides with the closure of its interior. Let $K: D \times D \mapsto \mathbb{C}$ be a function which is called a kernel. Then the linear operator $A: C(D) \mapsto C(D)$ defined by

$$
(A \varphi)(x):=\int_{D} K(x, y) \varphi(y) \mathrm{d} y, \quad x \in D,
$$

is called an integral operator with kernel K. For continuous kernel the operator is compact with norm

$$
\|A\|_{\infty}=\max _{x \in D} \int_{D}|K(x, y)| \mathrm{d} y .
$$

For weakly singular kernel the operator remains compact.

Having boundary value problems in scattering theory in mind, the integral operators need to be analysed on surfaces in $\mathbb{R}^{m}, m=2,3$. Therefore, a classification of smooth domains is introduced. First, the regularity of a domain can be described as in the following definition.

Definition A.1.4 (Classes of domains). An open and connected domain $D \in \mathbb{R}^{m}$ is of class $C^{k}, k \in \mathbb{N}$, if for each point $z$ of the boundary $\partial D$ there exists a neighbourhood $V_{z}$ of $z$ with the following properties:

- The intersection $V_{z} \cap \bar{D}$ can be mapped bijectiveley onto the half ball $\left\{x \in \mathbb{R}^{m}\right.$ : $\left.|x|<1, x_{m} \geq 0\right\}$.

- The mapping and its inverse are $k$-times continuously differentiable.

- The intersection $V_{z} \cap \partial D$ is mapped onto the disk $\left\{x \in \mathbb{R}^{m}:|x|<1, x_{m}=0\right\}$.

Further, denote by $C^{k}(D)$ the linear space of real or complex valued functions defined on the domain $D$ which are $k$-times continuously differentiable.

With the weakly singular kernels defined on a boundary the integral operator on a surface can be properly defined.

Definition A.1.5 (weakly singular, surface). A continuous kernel $K(x, y), x, y \in$ $\partial D, x \neq y$ is called weakly singular if there exists positive constants $C$ and $a \in$ $(0, m-1]$ such that

$$
|K(x, y)| \leq C|x-y|^{a-m+1}, \quad x, y \in \partial D, x \neq y .
$$

Theorem A.1.6 (Integral operator, surface). Let $D$ be a bounded open domain of class $C^{1}$ with the boundary denoted by $\partial D$. Then the integral operator $A: C(\partial D) \mapsto C(\partial D)$ defined as

$$
(A \varphi)(x):=\int_{\partial D} K(x, y) \varphi(y) \mathrm{d} s(y), \quad x \in \partial D
$$

with a continuous or weakly singular kernel $K$ is a compact operator.

Now, defining and analysing integral equations can be properly done. 


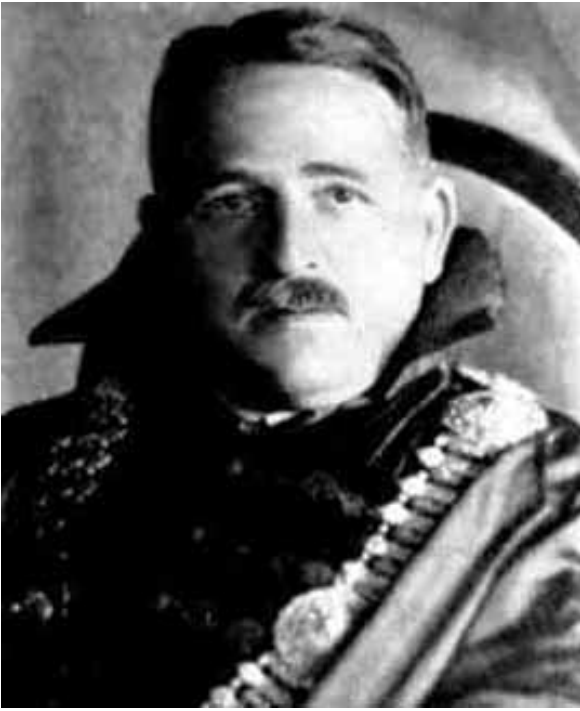

(a) Frigyes Riesz

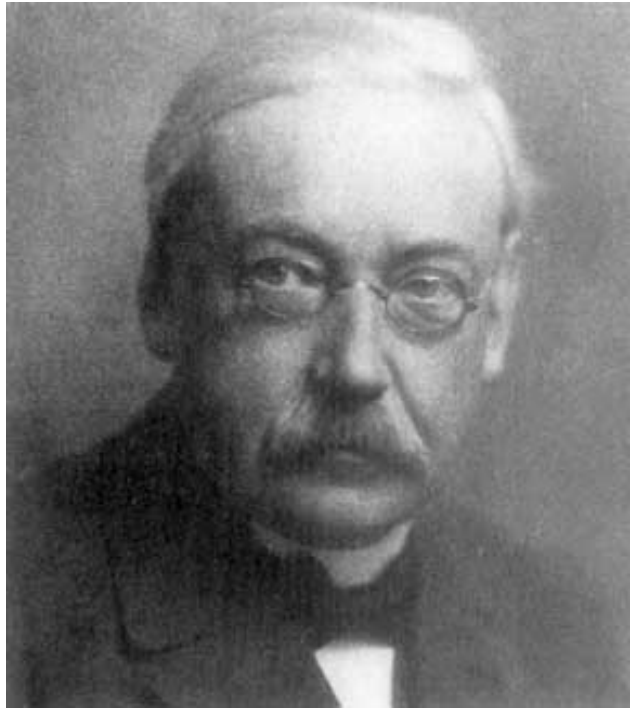

(b) Erik Ivar Fredholm

Figure A.1: Frigyes Riesz (1880 - 1956) and Erik Ivar Fredholm (1866 - 1927).

\section{A.2 Riesz-Fredholm theory}

Consider an operator equation of the second kind

$$
(I-A) \varphi=f
$$

with a compact linear operator $A: X \mapsto X$ defined on a normed space $X$. The theory for solving these equations was developed by Frigyes Riesz (1880 - 1956) in his work [42] and initiated through the work [12] of Erik Ivar Fredholm (1866 - 1927). Frigyes Riesz was a Hungarian mathematician. Erik Ivar Fredholm was a Swedish mathematician who had a fundamental impact on the theory of integral equations. From both a picture is shown in Figure A.1. The theory presented here follows [23, Chapter 3 and 4 ].

Some of the analysis done by Riesz for this kind of integral equations can be summed up in the following Theorem.

Theorem A.2.1 (Invertibility). Let $X$ be a normed space and the operator $A: X \mapsto X$ be compact and linear. Then the nullspace of the operator $I-A$

$$
N(I-A):=\{\varphi \in X:(I-A) \varphi=0\}
$$

is a finite dimensional subspace of $X$. Further, $I-A$ is injective if and only if it is surjective. If $I-A$ is injective (and therefore bijective), then the inverse operator $(I-A)^{-1}: X \mapsto X$ is bounded.

This Theorem can be reformulated in terms of solvability of an operator equation of the second kind. 
Theorem A.2.2 (Riesz - solvability). Let $X$ be a normed space and $A: X \mapsto X$ a linear compact operator. If the homogeneous equation

$$
(I-A) \varphi=0
$$

only has the trivial solution $\varphi=0$, then for each $f \in X$ the inhomogeneous equation

$$
(I-A) \varphi=f
$$

has a unique solution $\varphi \in X$ and this solution depends continuously on $f$. If the homogeneous equation (A.4) does have nontrivial solutions, then it has a finite number of linear independent solutions. Then the inhomogeneous equation (A.5) is either unsolvable or its general solution is a superposition of the linear independent solutions for the homogenous equation plus a particular solution of the inhomogeneous equation.

Proof. see Corollary 3.5 in [23].

The Riesz Theorem (Theorem A.2.2) implies that if $(I-A)$ is injective it is continuous invertible. Further, to show existence of a solution of (A.5) it is sufficient to consider the simpler problem wether (A.4) has only the trivial solution, which in scattering theory is usually established with a uniqueness proof of a corresponding scattering problem. The Theorem also does not make an explicit statement about the solvability of (A.5) if (A.4) does have nontrivial solutions. This question is settled by the Fredholm alternative [12], which will be presented now.

Theorem A.2.3 (Fredholm alternative). Let $f \subset \mathbb{R}^{m}$ be either a domain as in Theorem A.1.3 or a boundary $\partial D$ as in Theorem A.1.6 and let $K$ be a continuous or weakly singular kernel. Then $\langle C(D), C(D)\rangle$ is a dual system with the bilinear form

$$
\langle\varphi, \psi\rangle:=\int_{D} \varphi(x) \psi(x) \mathrm{d} x, \quad \varphi, \psi \in C(D) .
$$

The homogeneous equations are given by

$$
\begin{array}{ll}
\varphi(x)-\int_{D} K(x, y) \varphi(y) \mathrm{d} y=0, & x \in D \\
\psi(x)-\int_{D} K(y, x) \psi(y) \mathrm{d} y=0, & x \in D
\end{array}
$$

and the inhomogeneous equations are written as

$$
\begin{aligned}
& \varphi(x)-\int_{D} K(x, y) \varphi(y) \mathrm{d} y=f(x), \quad x \in D \\
& \psi(x)-\int_{D} K(y, x) \psi(y) \mathrm{d} y=g(x), \quad x \in D
\end{aligned}
$$

Then, there are two cases of solvability.

1. The homogenous integral equations only have the trivial solutions $\varphi=\psi=0$ and the inhomogeneous integral equations have a unique solution $\varphi, \psi$ for each right hand side $f, g$, respectively. 


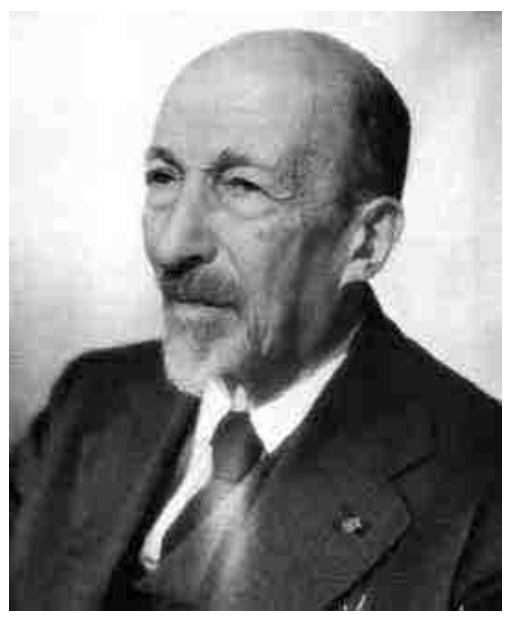

Figure A.2: Jacques Solomon Hadamard (1865 - 1963).

2. The homogeneous integral equations have the same finite number of linearly independent solutions and the inhomogeneous equations are solvable if and only if the righthand sides $f, g$ satisfy

$$
\begin{aligned}
& \int_{D} f(x) \psi(x) \mathrm{d} x=0 \\
& \int_{D} g(x) \varphi(x) \mathrm{d} x=0
\end{aligned}
$$

for all solutions $\psi, \varphi$ of the homogeneous equations respectively.

This establishes the theory for solvability of integral equations of the second kind.

\section{A.3 III-posed problems}

In inverse scattering theory the concept of ill-posedness for integral equations is in particular interest and is introduced here. The ill-posed solutions of these integral equations are either non-unique, non-existent or instable. First, to understand exactly what a ill-posed problem is, define a well-posed problem. Jacques Solomon Hadamard (1865 - 1963) introduced three properties for well-posed problems for boundary value problems for partial differential equations [15].

Definition A.3.1 (Well-posed problem). Let $A: U \mapsto V$ be a not necessarily linear operator from a subset $U$ of a normed space $X$ into a subset $V$ of a normed space $Y$ and consider the operator equation

$$
A \varphi=f
$$

Then, a well-posed problem has the three properties:

a) Existence. $A$ is surjective. 
b) Uniqueness. $A$ is injective.

c) Continuous dependence of the solution on the data. The inverse operator $A^{-1}: V \mapsto$ $U$ exists and is continuous.

If one of the three properties is not fulfilled, (A.6) is called an ill-posed problem.

According to this Definition, there are three types of ill-posedness. If a) is not fulfilled there is no solution (nonexistence), if $\mathrm{b}$ ) is not fulfilled there may be more than one solution (nonuniqueness) and if c) is not fulfilled the solution of (A.6) does not depend continuously on the data $f$ (instability). The case of instability is of primary interest in the study of applied ill-posed problems and is the only case to occur in this work.

Theorem A.3.2 (Ill-posedness of equations of the first kind). Let $A: X \mapsto Y$ be a linear compact operator from a normed space $X$ into a normed space $Y$. If $X$ is of infinite dimension the equation of the first kind $A \varphi=f$ is ill-posed.

Proof. Assume that $A^{-1}: X \mapsto Y$ exists and is continuous. Then, consider $I=A^{-1} A$. The product of a continuous and a compact operator is compact (see [23, Theorem 2.16]), hence the Identity operator is compact. From Theorem 2.20 in [23] it follows that $X$ must be finite dimensional and therefore $A^{-1}$ cannot be continuous and then the equation of the first kind is ill-posed.

\section{A.4 Tikhonov regularisation}

An ill-posed equation is numerically unstable and has to be treated properly, since inevitable round-off errors are amplified. A numerical approximation to a given equation may be viewed as the solution to perturbed data. Because $A^{-1}$ is noncontinuous, a straight forward numerical implementation of the equation would then lead to a very bad numerical approximation to the solution. This means that the condition number of the numerical approximation matrix for $A$ would grow with the quality of the discretisation, i.e. the approximation to the equation of the first kind would become worse.

The numerical approximation can be viewed as having pertubed data $f^{\delta}$. Given a perturbed right hand side $f^{\delta}$ with known error level

$$
\left\|f^{\delta}-f\right\| \leq \delta
$$

it cannot be expected that $f^{\delta} \in A(X)$, the range of $A$. This means it is necessary to find a good approximate and stable solution $\varphi^{\delta}$ to the true solution $\varphi$, i.e. $\varphi^{\delta}$ depends continuously on the data $f^{\delta}$. Stable methods for constructing an approximate solution to an ill-posed problem are called regularisation schemes and are presented in the following Definition. 
Definition A.4.1 (Regularisation scheme). Let $X$ and $Y$ be normed spaces and let $A: X \mapsto Y$ be an injective bounded linear operator. Then a family of bounded linear operators $R_{\alpha}: Y \mapsto X, \alpha>0$ with the property of pointwise convergence

$$
\lim _{\alpha \rightarrow 0} R_{\alpha} \varphi=A^{-1} A \varphi=\varphi
$$

for all $\varphi \in X$ is called a regularisation scheme for the operator $A$. The parameter $\alpha$ is called the regularisation parameter.

Remark. It can be shown that $R_{\alpha}$ can not be uniformly bounded with respect to $\alpha$.

For analysing the approximation error, first write down the regularised solution

$$
\varphi_{\alpha}^{\delta}:=R_{\alpha} f^{\delta},
$$

which comes from applying the regularisation scheme to the equation $A \varphi^{\delta}=f^{\delta}$. For looking at the approximation error first take the difference of the solutions

$$
\varphi_{\alpha}^{\delta}-\varphi=R_{\alpha} f^{\delta}-R_{\alpha} f+R_{\alpha} A \varphi-\varphi .
$$

Then, using the triangle inequality leads to the estimate

$$
\left\|\varphi_{\alpha}^{\delta}-\varphi\right\| \leq \delta\left\|R_{\alpha}\right\|+\left\|R_{\alpha} A \varphi-\varphi\right\| .
$$

Thus, the error consists of two parts. The first term reflects the influence of the incorrect data and the second term can be seen as the approximation error between $R_{\alpha}$ and $A^{-1}$. With $\alpha \rightarrow 0$ the first term is increasing due to the remark after Regularisation schemes (Definition A.4.1) and the second term is decreasing due to (A.7). Hence, every regularisation scheme and its application has to carefully adjust $\alpha$ in dependence of the problem and the error level $\delta$ to have a low total error level for the regularised solution. A regularisation strategy should have the property that the approximate solution converges to the true solution, when the error level tends to zero, which is summed up in the following Definition.

Definition A.4.2 (Regular strategy). A strategy for a regularisation scheme $R_{\alpha}, \alpha>0$ is a rule for the choice of the regularisation parameter $\alpha=\alpha(\delta)$ depending on the error level $\delta$. A strategy is called regular if for all $f \in A(X)$ and all $f^{\delta} \in Y$ with $\left\|f^{\delta}-f\right\| \leq \delta$ it holds that

$$
\lim _{\delta \rightarrow 0} R_{\alpha} f^{\delta}=A^{-1} f
$$

There are a number of different regularisation methods which have been developed in the literature. In this work the Tikhonov regularisation is used, which is presented next. The method is named after Andrey Nikolayevich Tychonoff (1906 - 1993) who was a Russian mathematician with a wide range of mathematical contributions. In particular in the scope of this work his book [44] is worth mentioning. 
Appendix A Integral equations

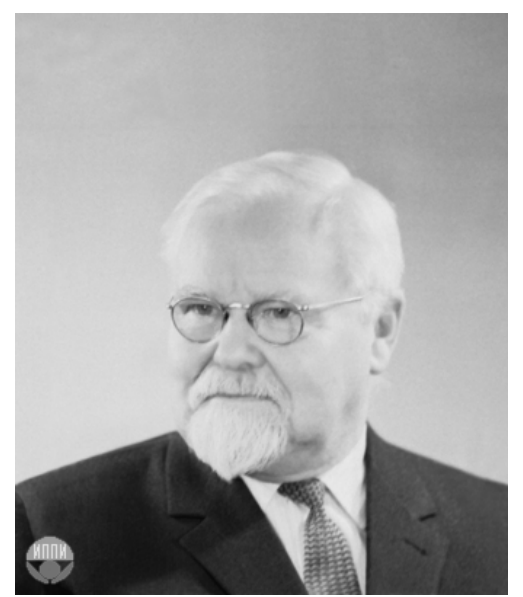

Figure A.3: Andrey Nikolayevich Tychonoff (1906 - 1993).

Theorem A.4.3 (Tikhonov regularisation). Let $A: X \mapsto Y$ be a compact linear operator. Then for each $\alpha>0$ the operator $\left(\alpha I+A^{*} A\right): X \mapsto X$ is bijective and has a bounded inverse. Furthermore, if $A$ is injective then

$$
R_{\alpha}:=\left(\alpha I+A^{*} A\right)^{-1} A^{*}
$$

describes a regularisation scheme with $\left\|R_{\alpha}\right\| \leq \frac{1}{2 \sqrt{\alpha}}$.

Proof. See proof of Theorem 15.23 in [23] 


\section{Appendix B}

\section{Common tools for scattering theory}

In this chapter some common tools for acoustic and electromagnetic scattering are introduced. First, Green's Theorem is described in Section B.1. The fundamental solutions for the acoustic and electromagnetic problem are using Hankel- and Bessel functions respectively, which are briefly discussed in Section B.3.

\section{B.1 Green's theorem and formula}

One of the basic tools for studying the Helmholtz equation are the Green's theorems and the Green's formula which originate from George Green (1793-1841). The original title page of the essay of Green containing Green's theorem is shown in Figure B.1.

Theorem B.1.1 (Green's theorem). Let $D$ be a bounded domain of class $C^{1}$ and let $v$ denote the unit normal vector to the boundary $\partial D$ directed into the exterior of $D$. Then, for $u \in C^{1}(\bar{D})$ and $v \in C^{2}(\bar{D})$ Green's first theorem is given by

$$
\int_{D}\{u \Delta v+\operatorname{grad} u \cdot \operatorname{grad} v\} \mathrm{d} x=\int_{\partial D} u \frac{\partial v}{\partial v} \mathrm{~d} s
$$

and for $u, v \in C^{2}(\bar{D})$ Green's second theorem is given by

$$
\int_{D}(u \Delta v-v \Delta u) \mathrm{d} x=\int_{\partial D}\left(u \frac{\partial v}{\partial v}-v \frac{\partial u}{\partial v}\right) \mathrm{d} s
$$

Proof. Applying Gauss divergence theorem to ( $u \operatorname{grad} v)$ proves (B.I). Interchanging $u$ and $v$ and subtracting the resulting equation from (B.1) proves (B.2).

A consequence of Green's theorems which is of interest here is Green's formula, which is also known as Helmholtz representation and is introduced in the next Theorem. It leads to some special properties of the Green's formula with respect to the Helmholtz equation which are used later on.

With the Helmholtz equation (Definition 2.1.1) Green's formula can be properly introduced.

Theorem B.1.2 (Green's formula / Helmholtz representation). Let $D$ be a bounded domain of class $C^{2}$ and let $v$ denote the unit normal vector to the boundary $\partial D$ directed into the exterior of $D$. Let $u \in C^{2}(D) \cap C(\bar{D})$ and $v \in C^{2}\left(\mathbb{R}^{3} \backslash \bar{D}\right) \cap C\left(\mathbb{R}^{3} \backslash D\right)$ be solutions to 
A N ESSAY

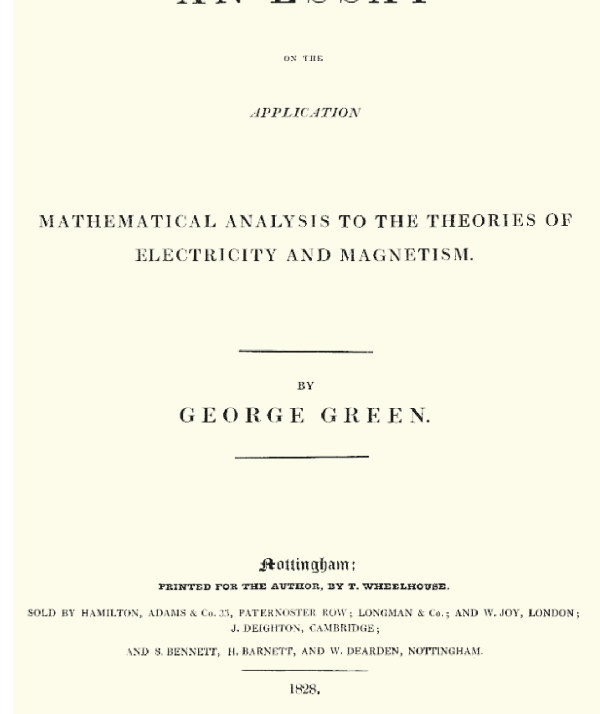

Figure B.1: Original essay on Green's theorem by George Green (1793 - 1841).

the Helmholtz equation which possess normal derivatives on the boundary in the sense that the limits

$$
\begin{array}{ll}
\frac{\partial u}{\partial v}(x)=\lim _{h \rightarrow+0} v(x) \cdot \operatorname{grad} u(x-h v(x)), & x \in \partial D \\
\frac{\partial v}{\partial v}(x)=\lim _{h \rightarrow+0} v(x) \cdot \operatorname{grad} v(x+h v(x)), & x \in \partial D
\end{array}
$$

exists uniformly on $\partial D$. Assume further that $v$ is radiating, then Green's formula

$$
\begin{aligned}
& u(x)=\int_{\partial D}\left\{\frac{\partial u}{\partial v}(y) \Phi(x, y)-u(y) \frac{\partial \Phi(x, y)}{\partial v(y)}\right\} \mathrm{d} s(y), \quad x \in D \\
& v(x)=\int_{\partial D}\left\{v(y) \frac{\partial \Phi(x, y)}{\partial v(y)}-\frac{\partial v}{\partial v}(y) \Phi(x, y)\right\} \mathrm{d} s(y), \quad x \in \mathbb{R}^{3} \backslash \bar{D}
\end{aligned}
$$

holds, where the volume integrals exist as improper integrals.

Proof. case $x \in D$ : Let $\partial D_{h}$ be a parallel surface ([9],[23]). Define the sphere contained in $D_{h}$ as $\Omega(x ; R):=\left\{y \in \mathbb{R}^{m}:|x-y|=R\right\}$ and direct the normal into the interior of $\Omega(x ; R)$. Now apply Green's second theorem (Theorem B.1.1) to the functions $u$ and $\Phi(x, \cdot)$ in the domain $D_{R}:=\left\{y \in D_{h}:|x-y|>R\right\}$ to obtain

$$
\int_{\partial D_{h} \cup \Omega(x ; R)}\left\{\frac{\partial u}{\partial v}(y) \Phi(x, y)-u(y) \frac{\partial \Phi(x, y)}{\partial v(y)}\right\} \mathrm{d} s(y)=0 .
$$

Using the fundamental solution and its derivative together with the mean value theorem in $\Omega(x ; R)$ leads to

$$
\lim _{R \rightarrow 0} \int_{\Omega(x ; R)}\left\{u(y) \frac{\partial \Phi(x, y)}{\partial v(y)}-\frac{\partial u}{\partial v}(y) \Phi(x, y)\right\} \mathrm{d} s(y)=u(x) .
$$


Then passing to the limit $h \rightarrow 0$ from the parallel surface finishes the proof for the case $x \in D$.

case $x \in \mathbb{R}^{m} \backslash \bar{D}$ : First, show that

$$
\int_{\Omega_{R}}|u|^{2} \mathrm{~d} s=O(1), \quad r \rightarrow \infty
$$

where $\Omega_{R}$ denotes the sphere of radius $R$ with center in the origin. Consider

$$
\int_{\Omega_{R}}\left|\frac{\partial u}{\partial v}-i \kappa u\right|^{2} \mathrm{~d} s
$$

where $v$ is the outward normal to $D_{R}$. From the radiation condition (Definition 2.1.2) it follows that

$$
\lim _{R \rightarrow \infty} \int_{\Omega_{R}}\left\{\left|\frac{\partial u}{\partial v}\right|^{2}+\kappa^{2}|u|^{2}+2 \kappa \Im\left(u \frac{\partial \bar{u}}{\partial v}\right)\right\} \mathrm{d} s=\int_{\Omega_{R}}\left|\frac{\partial u}{\partial v}-i \kappa u\right|^{2} \mathrm{~d} s=0 .
$$

For sufficiently large $R$ apply Green's first Theorem (Theorem B.1.1) in the domain $D_{R}:=\left\{y \in \mathbb{R}^{m} \backslash \bar{D}:|y|<r\right\}$ to obtain

$$
\int_{\Omega_{r}} u \frac{\partial \bar{u}}{\partial v} \mathrm{~d} s=\int_{\partial D} u \frac{\partial \bar{u}}{\partial v} \mathrm{~d} s-\kappa^{2} \int_{D_{R}}|u|^{2} \mathrm{~d} y+\int_{D_{R}}|\operatorname{grad} u|^{2} \mathrm{~d} y
$$

Taking the imaginary part of (B.6) and inserting in (B.5) leads to

$$
\lim _{r \rightarrow \infty} \int_{\Omega_{R}}\left\{\left|\frac{\partial u}{\partial v}\right|^{2}+\kappa^{2}|u|^{2}\right\} \mathrm{d} s=-2 \kappa \Im \int_{\partial D} u \frac{\partial \bar{u}}{\partial v} \mathrm{~d} s
$$

Both terms on the left hand side of (B.7) are nonnegative and therefore the terms has to be individually bounded for $r \rightarrow \infty$ since the right hand side is bounded. Hence, (B.4) is proven. Now from (B.4) and the radiation condition it follows that

$$
\frac{\partial \Phi(x, y)}{\partial v(y)}-i \kappa \Phi(x, y)=O\left(\frac{1}{r^{2}}\right), \quad r \rightarrow \infty
$$

which is valid uniformly for $y \in \Omega_{R}$. Then, applying the Cauchy-Schwarz inequality leads to

$$
I_{1}:=\int_{\Omega_{R}} u(y)\left\{\frac{\partial \Phi(x, y)}{\partial v(y)}-i \kappa \Phi(x, y)\right\} \mathrm{d} s(y) \rightarrow 0, \quad r \rightarrow \infty
$$

Similar, from the radiation condition and $\Phi(x, y)=O(1 / r)$ for $y \in \Omega_{R}$ and again applying Cauchy-Schwarz yields

$$
I_{2}:=\int_{\Omega_{R}} \Phi(x, y)\left\{\frac{\partial u}{\partial v}(y)-i \kappa u(y)\right\} \mathrm{d} s(y) \rightarrow 0, \quad r \rightarrow \infty
$$

Then

$$
\int_{\Omega_{R}}\left\{u(y) \frac{\partial \Phi(x, y)}{\partial v(y)}-\frac{\partial u}{\partial v}(y) \Phi(x, y)\right\} \mathrm{d} s(y)=I_{1}-I_{2} \rightarrow 0, \quad r \rightarrow \infty
$$

from which the proof is completed applying the first case for $D_{R}$ and taking the limit $r \rightarrow \infty$. 


\section{B.2 Stratton-Chu formulas}

Here, the equivalent to Green's formula (Theorem B.1.2) in the case of Maxwell equations is presented.

Theorem B.2.1 (Stratton-Chu formulas). Let D be a bounded obstacle with open complement of class $C^{2}$ with normal vector $v$ directed into the exterior (as in Definition 6.1.1).

1. Let $E, H \in C^{1}\left(\mathbb{R}^{3} \backslash \bar{D}\right) \cap C\left(\mathbb{R}^{3} \backslash D\right)$ be radiating solutions to the Maxwell equations (6.4). Then for $x \in \mathbb{R}^{3} \backslash \bar{D}$ the Stratton-Chu formulas are given by

$$
\begin{aligned}
E(x)= & \operatorname{curl} \int_{\partial D} \nu(y) \times E(y) \Phi(x, y) \mathrm{d} s(y) \\
& -\frac{1}{i \kappa} \text { curl curl } \int_{\partial D} v(y) \times H(y) \Phi(x, y) \mathrm{d} s(y)
\end{aligned}
$$

and

$$
\begin{aligned}
H(x)= & \operatorname{curl} \int_{\partial D} v(y) \times H(y) \Phi(x, y) \mathrm{d} s(y) \\
& +\frac{1}{i \kappa} \operatorname{curlcurl} \int_{\partial D} v(y) \times E(y) \Phi(x, y) \mathrm{d} s(y) .
\end{aligned}
$$

2. Let $E, H \in C^{1}(D) \cap C(\bar{D})$ be solutions to the Maxwell equations. Then for $x \in D$ the Stratton-Chu formulas are given by the formulas (B.8) and (B.9) where the right hand sides are multiplied with $(-1)$.

Proof. A proof for case 1 is the proof for Theorem 6.6 in [8] and for case 2 a proof is equivalent to the proof of Theorem 6.2 in [8].

Remark. The Theorem covers only the case of homogeneous background medium, but the formulas can be analogous be derived for a layered background medium. The Stratton Chu formulas are distributions of magnetic and electric dipoles over the boundary and as such justify that (6.20) can be seen as a fundamental solution to the time harmonic Maxwell equations.

\section{B.3 Spherical Bessel and Hankel functions}

In this work, spherical Bessel functions are extensively used for the Green's tensor in a two-layered medium in electromagnetic scattering. Also, Hankel functions are used for the two-dimensional fundamental solution for the Helmholtz equation, i.e. for the acoustic case.

Theorem B.3.1 (Spherical Bessel differential equation). Provided $f$ solves the spherical Bessel differential equation given as

$$
t^{2} f^{\prime \prime}(t)+2 t f^{\prime}+\left[t^{2}-n(n+1)\right] f(t)=0,
$$




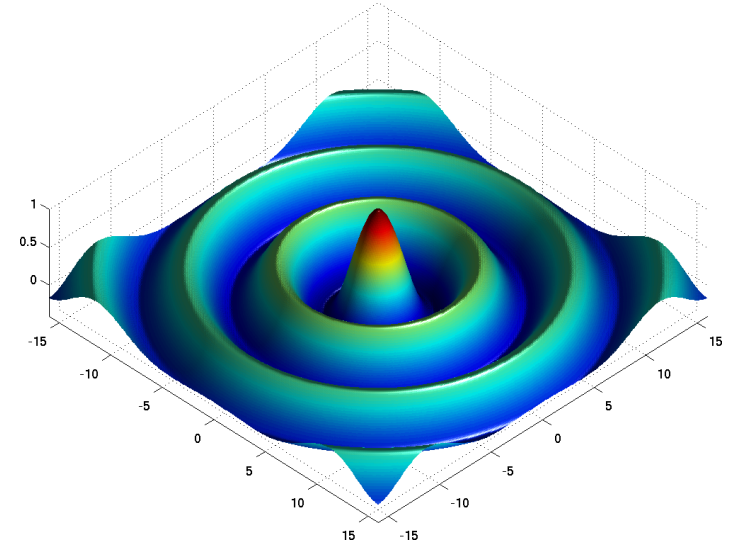

(a) $J_{0}$

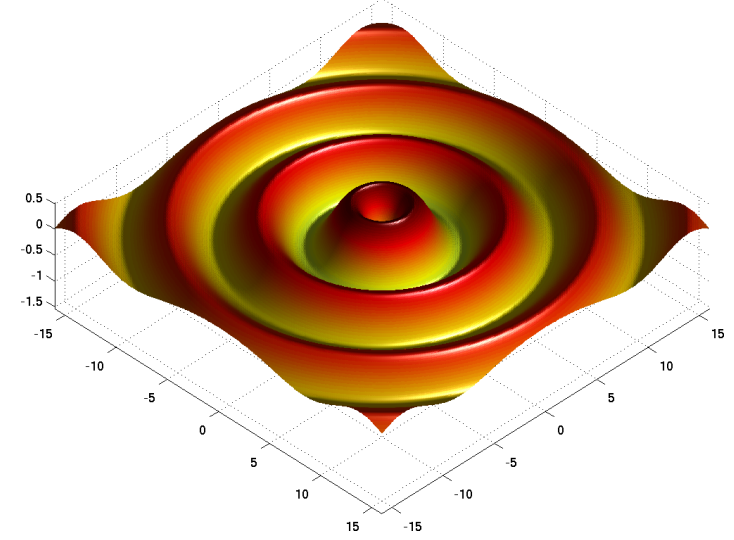

(b) $N_{0}$

Figure B.2: Bessel functions of order 0.

with $n \in \mathbb{N}$, then

$$
u(x)=f(\kappa|x|) Y_{n}(\hat{x})
$$

solves the Helmholtz equation, where $Y_{n}$ are the spherical harmonics of order $n$ and $\hat{x}=$ $x /|x|$.

Theorem B.3.2 (Spherical Bessel, Neumann and Hankel functions). Let $n \in \mathbb{N}$. Then, the spherical Bessel functions

$$
J_{n}(t)=\sum_{p=0}^{\infty} \frac{(-1)^{p} t^{n+2 p}}{2^{p} p ! 1 \cdot 3 \cdots(2 n+2 p+1)}
$$

and the spherical Neumann functions

$$
N_{n}(t)=-\frac{(2 n) !}{2^{n} n !} \sum_{p=0}^{\infty} \frac{(-1)^{p} t^{2 p-n-1}}{2^{p} p !(-2 n+1)(-2 n+3) \cdots(-2 n+2 p-1)}
$$

represent solutions to the spherical Bessel differential equation (Theorem B.3.1). $I_{n}$ is an analytic function in $\mathbb{R}$ and $N_{n}$ is analytic in $(0, \infty)$. The following linear combinations are called the spherical Hankel functions

$$
H_{n}^{(1)}=J_{n}+i N_{n}, \quad H_{n}^{(2)}=J_{n}-i N_{n}
$$

of the first and second kind of order $n$.

The Bessel functions have some recurrence relations and properties which are useful in this work and are summed up here. 
Theorem B.3.3 (Bessel functions - Recurrence relations). The following recurrence relations hold for the spherical Bessel functions of order $n$.

$$
\begin{aligned}
\frac{2 n}{x} J_{n}(x) & =J_{n-1}(x)+J_{n+1}(x) \\
J_{-n}(x) & =(-1)^{n} J_{n}(x) \\
2 J_{n}^{\prime}(x) & =J_{n-1}(x)-J_{n+1}(x) \\
J_{n+1}(x) & =\frac{n}{x} J_{n}(x)-J_{n}^{\prime}(x) \\
J_{n-1}(x) & =\frac{n}{x} J_{n}(x)+J_{n}^{\prime}(x)
\end{aligned}
$$

Because integrals over Bessel functions have some broader application they were given the name Hankel transforms and are examined in the following.

Definition B.3.4 (Hankel transform). For the given function $f:[0, \infty) \mapsto \mathbb{C}$ the function $g:[0, \infty) \mapsto \mathbb{C}$ defined by

$$
g(r):=\int_{0}^{\infty} f(\lambda) J_{n}(\lambda r) \lambda \mathrm{d} \lambda
$$

with Bessel-functions $J_{n}$ (see Section B.3) of order $n \in \mathbb{N}_{0}$, is called the Hankel transform of $f$.

Since there exist some different normalisations of the Fourier transform it is defined here.

Definition B.3.5 (Fourier transform). Let $f \in L^{1}\left(\mathbb{R}^{m}\right)$ be a function. Then the Fourier transform is defined as

$$
\hat{f}(y):=\mathcal{F}(f)(y):=\frac{1}{(2 \pi)^{m / 2}} \int_{\mathbb{R}^{m}} e^{-i x \cdot y} f(x) \mathrm{d} x .
$$

The inverse Fourier transform as

$$
\mathcal{F}^{-1}(f)(y):=\frac{1}{(2 \pi)^{m / 2}} \int_{\mathbb{R}^{m}} e^{i x \cdot y} f(x) \mathrm{d} x .
$$

The fundamental solution of the Helmholtz equation (Definition 2.2.1) can be expressed through a Hankel transform which is called the Sommerfeld integral or Sommerfeld identity [43, Page 242].

Theorem B.3.6 (Sommerfeld integral). Let $x, y \in \mathbb{R}^{3}, x \neq y$ and abbreviate the radius as $r:=\sqrt{\left(x_{1}-y_{1}\right)^{2}+\left(x_{2}-y_{2}\right)^{2}}$. Then, the fundamental solution $\Phi$ of the Helmholtz equation for wave number $\kappa$ with $\Im \kappa>0$ can be expressed as the Sommerfeld integral

$$
\Phi(x, y)=\frac{1}{4 \pi} \int_{0}^{\infty} \frac{\lambda}{\alpha} e^{-\alpha\left|x_{3}-y_{3}\right|} J_{0}(\lambda r) \mathrm{d} \lambda
$$


with $\alpha:=\sqrt{\lambda^{2}-\kappa^{2}}$ and using the branch $\sqrt{-1}=-i$ of the square root which corresponds to $\Re \alpha>0$.

Proof. The idea is to analyse a cylindrical function with the Fourier transform. Let $\mathfrak{f}: \mathbb{R}^{2} \backslash\{0\} \mapsto \mathbb{C}$ be a function which in polar coordinates

$$
\lambda_{1}=r \cos \varphi, \quad \lambda_{2}=r \sin \varphi,
$$

has the property

$$
\mathfrak{f}\left(\lambda_{1}, \lambda_{2}\right)=\breve{f}(r) e^{i n \varphi}
$$

with $n \in \mathbb{N} \cup\{0\}$. Using the two-dimensional Fourier transform for $\lambda_{1}$ and $\lambda_{2}$ yields

$$
\mathfrak{f}\left(\lambda_{1}, \lambda_{2}\right)=\frac{1}{4 \pi^{2}} \int_{-\infty}^{\infty} \int_{-\infty}^{\infty} \int_{-\infty}^{\infty} \int_{-\infty}^{\infty} \mathfrak{f}(\xi, \eta) e^{i \beta\left(\lambda_{1}-\xi\right)} e^{i \gamma\left(\lambda_{2}-\eta\right)} \mathrm{d} \xi \mathrm{d} \eta \mathrm{d} \beta \mathrm{d} \gamma
$$

With the polar coordinates

$$
\begin{array}{ll}
\xi=\rho \cos \psi, & \beta=\sigma \cos \omega \\
\eta=\rho \sin \psi, & \gamma=\sigma \sin \omega
\end{array}
$$

and the trigonometric identity

$$
\cos (x-y)=\cos (x) \cos (y)+\sin (x) \sin (y)
$$

this becomes

$$
\breve{\mathfrak{f}}(r) e^{i n \varphi}=\frac{1}{4 \pi^{2}} \int_{0}^{\infty} \int_{-\pi}^{\pi} \int_{0}^{\infty} \int_{-\pi}^{\pi} \breve{\mathfrak{f}}(\rho) e^{i n \psi} e^{i \sigma r \cos (\omega-\varphi)} e^{-i \sigma \rho \cos (\psi-\omega)} \rho \mathrm{d} \psi \mathrm{d} \rho \sigma \mathrm{d} \omega \mathrm{d} \sigma .
$$

Dividing the equation through $e^{i n \varphi}$ yields

$$
\begin{aligned}
\breve{\mathfrak{f}}(r)=\int_{0}^{\infty} \int_{-\pi}^{\pi} \int_{0}^{\infty} \breve{\mathfrak{f}}(\rho) \frac{1}{2 \pi} \int_{-\pi}^{\pi} e^{-i \sigma \rho \cos (\psi-\omega)} e^{i n\left(\psi-\omega+\frac{\pi}{2}\right)} \rho \mathrm{d} \psi \mathrm{d} \rho \\
\frac{1}{2 \pi} e^{i \sigma r \cos (\omega-\varphi)} e^{i n\left(\omega-\varphi-\frac{\pi}{2}\right)} \sigma \mathrm{d} \omega \mathrm{d} \sigma .
\end{aligned}
$$

Using the integral representation of the Bessel functions ([46, Page 31])

$$
\begin{aligned}
J_{n}(z) & =\frac{1}{2 \pi} \int_{-\pi}^{\pi} e^{i z \cos a} e^{i n\left(a-\frac{\pi}{2}\right)} \mathrm{d} a \\
& =\frac{1}{2 \pi} \int_{-\pi}^{\pi} e^{-i z \cos b} e^{i n\left(b+\frac{\pi}{2}\right)} \mathrm{d} b
\end{aligned}
$$

and the substitution

$$
a=\omega-\varphi, \quad b=\psi-\omega
$$

the equation becomes

$$
\breve{\mathfrak{f}}(r)=\int_{0}^{\infty} \int_{0}^{\infty} \breve{\mathfrak{f}}(\rho) J_{n}(\sigma \rho) \rho \mathrm{d} \rho J_{n}(\sigma r) \sigma \mathrm{d} \sigma .
$$


This can be written as the system

$$
\begin{aligned}
\breve{f}(r) & =\int_{0}^{\infty} \mathfrak{g}(\sigma) J_{n}(\sigma r) \sigma \mathrm{d} \sigma & , r \in(0, \infty) \\
\mathfrak{g}(\sigma) & =\int_{0}^{\infty} \breve{\mathfrak{f}}(\rho) J_{n}(\sigma \rho) \rho \mathrm{d} \rho & , \sigma \in(0, \infty) .
\end{aligned}
$$

Now, leaving the general separation and choosing the function $\breve{f}$ as

$$
\breve{f}(r)=\frac{e^{i \kappa r}}{r},
$$

with $y \in \mathbb{R}^{3}$ be fixed, $x_{3}=y_{3}$ and $r>0$. Further, renaming $\lambda=\sigma, f=\mathfrak{g}$ and choosing $n=0$ in (B.25) the system changes to

$$
\begin{gathered}
\frac{e^{i \kappa r}}{r}=\int_{0}^{\infty} f(\lambda) J_{0}(\lambda r) \lambda \mathrm{d} \lambda \quad, r \in(0, \infty) \\
f(\lambda)=\int_{0}^{\infty} e^{i \kappa r} J_{0}(\lambda \rho) \mathrm{d} r \quad, \lambda \in(0, \infty) .
\end{gathered}
$$

The integral in (B.26) is well defined since $\Im(\kappa)>0$. Now, the fundamental solution $\Phi$ can be written as

$$
\Phi(r)=\frac{1}{4 \pi} \frac{e^{i \kappa r}}{r}=\frac{1}{4 \pi} \int_{0}^{\infty} \lambda f(\lambda) J_{0}(\lambda r) \mathrm{d} \lambda
$$

Inserting the integral representation of the Bessel function $J_{0}$ leads to

$$
f(\lambda)=\frac{1}{2 \pi} \int_{-\pi}^{\pi} \int_{0}^{\infty} e^{i \rho(\kappa+\lambda \cos \omega)} \mathrm{d} \rho \mathrm{d} \omega
$$

Again using the property $\Im(\kappa)>0$ gives

$$
f(\lambda)=-\frac{1}{2 \pi i} \int_{-\pi}^{\pi} \frac{1}{\kappa+\lambda \cos \omega} \mathrm{d} \omega
$$

Then, the integral [14, Page 99] now leads to

$$
\begin{aligned}
f(\lambda) & =-\frac{1}{2 \pi i} \frac{2 \pi}{\sqrt{\kappa^{2}-\lambda^{2}}} \\
& =\frac{1}{\alpha},
\end{aligned}
$$

which is an explicit form of $f$. Finally, the fundamental solution for $x_{3}=y_{3}$ and $r>0$ can be represented as

$$
\Phi(r)=\frac{1}{4 \pi} \int_{0}^{\infty} \frac{\lambda}{\alpha} J_{0}(\lambda r) \mathrm{d} \lambda, r \in(0, \infty) .
$$

For the case $x_{3}>y_{3}$ the following ansatz will be used: Let $\lambda \in(0, \infty)$ be fixed and define

$$
g\left(x_{1}, x_{2}\right):=\frac{\lambda}{\alpha} J_{0}(\lambda r)
$$


Then find a function $h: \mathbb{R} \rightarrow \mathbb{C}$ with the property $h\left(y_{3}\right)=1$ such that the function $F\left(x_{1}, x_{2}, x_{3}\right):=g\left(x_{1}, x_{2}\right) h\left(x_{3}\right)$ fullfills the Helmholtz equation for a wave number $\kappa$ and $F$ tends to zero for $x_{3} \rightarrow \infty$. Then, the function $h$ has to solve the boundary value problem

$$
\begin{aligned}
h^{\prime \prime}\left(x_{3}\right) & =\left(\lambda^{2}-\kappa^{2}\right) h\left(x_{3}\right) \\
h\left(y_{3}\right) & =1 \\
h\left(x_{3}\right) & \rightarrow 0 \quad, x_{3} \rightarrow \infty .
\end{aligned}
$$

The unique solution to this problem is

$$
h\left(x_{3}\right)=e^{-\alpha\left(x_{3}-y_{3}\right)}
$$

For the case $x_{3}<y_{3}$ analogously the solution is

$$
h\left(x_{3}\right)=e^{\alpha\left(x_{3}-y_{3}\right)} .
$$

Thus, for $x, y \in \mathbb{R}^{3}$ with $r>0$ and using $F$, the fundamental solution can be represented as

$$
\Phi(r)=\frac{e^{i \kappa|x-y|}}{4 \pi|x-y|}=\frac{1}{4 \pi} \int_{0}^{\infty} \frac{\lambda}{\alpha} e^{-\alpha\left|x_{3}-y_{3}\right|} J_{0}(\lambda r) \mathrm{d} \lambda .
$$

For the case $r=0$ for $x_{3} \neq y_{3}$ elementary integration yield

$$
\frac{1}{4 \pi} \int_{0}^{\infty} \frac{\lambda}{\alpha} e^{-\alpha\left|x_{3}-y_{3}\right|} \mathrm{d} \lambda=\frac{e^{i \kappa|x-y|}}{4 \pi|x-y|}=\Phi(|x-y|),
$$

the existence of the integral is ensured through $\Re \alpha>0$.

Remark. The Theorem remains valid for the limit $\Im \mathcal{K} \rightarrow 0$. The physical interpretation of the Sommerfeld identity is that a spherical wave can be expanded into a summation of cylindrical waves in $r$ direction, multiplied by a plane wave in the $z$ direction. 
Appendix B Common tools for scattering theory

138 


\section{Appendix C}

\section{Numerical treatment}

In this work integral equations of the second kind are employed. The numerical solution of such equations needs proper handling of the integral operators. An Nyström method is chosen to handle the operators throughout this work.

\section{C.1 Nyström method}

Consider numerical integration of an integral operator of the form

$$
(A \varphi)(x)=\int_{\partial D} K(x, y) \varphi(y) \mathrm{d} y, \quad x \in \partial D
$$

with continuous or weakly singular kernel $K$. Then, with the points $x_{k}, k=1, \ldots, n$ an approximation to the integral operator through a quadrature rule is given by

$$
\left(A_{n} \varphi\right)(x):=\sum_{k=1}^{n} \alpha_{k} K\left(x, x_{k}\right) \varphi\left(x_{k}\right), \quad x \in \partial D .
$$

Note that the $x_{k}$ are also dependent on the total number of points $n$ which for simplicity is neglected in the notation. The solution to the integral equation of the second kind

$$
\varphi-A \varphi=f
$$

is then approximated by the solution of

$$
\varphi_{n}-A_{n} \varphi_{n}=f
$$

This now can be reduced to a finite-dimensional linear system. The Nyström method now consists of interpolating the density $\varphi$ on the quadrature points which is written up in the following theorem. The method has its name from the mathematician Evert J. Nyström (1895-1960) and was published in [31] and the representation here follows [8].

Theorem C.1.1 (Nyström method). Consider the semi-discretized integral equation of the second kind

$$
\varphi_{n}(x)-\sum_{k=1}^{n} \alpha_{k} K\left(x, x_{k}\right) \varphi_{n}\left(x_{k}\right)=f(x), \quad x \in \partial D .
$$


Then the values $\varphi_{n, j}=\varphi_{n}\left(x_{j}\right), j=1, \ldots, n$ at the quadrature points satisfy the linear system

$$
\varphi_{n, j}-\sum_{k=1}^{\infty} \alpha_{k} K\left(x_{j}, x_{k}\right) \varphi_{n, k}=f\left(x_{j}\right), j=1, \ldots, n .
$$

Conversely, let the $\varphi_{n, j}=\varphi_{n}\left(x_{j}\right), j=1, \ldots, n$ be a solution of the (C.2). Then, the function $\varphi_{n}$ defined by

$$
\varphi_{n}(x):=f(x)+\sum_{k=1}^{n} \alpha_{k} K\left(x, x_{k}\right) \varphi_{n, k}, \quad x \in \partial D
$$

solves (C.1). Equation (C.3) is called the Nyström interpolation formula.

Remark. This especially means that the solutions $\varphi_{n}$ of (C.I) are completely determined by their values at the nodes $\left\{x_{j}\right\}$.

For the numerical calculations the Nyström method is used with trapezoidal rule with ignoring the singularity. This is a further approximation to the operator but which can be shown to be convergent to the original. This hasn't a good convergence rate but trapezoidal rules with better convergence rates in this weakly singular case and in higher dimensions is complex and neglected in this work.

\section{C.2 Fast Hankel transform}

The fast Hankel transform is used for fast numerical calculations of Hankel integrals. These are extensively used for the Green's tensor for a two-layered medium in a setting for time-harmonic electromagnetic waves (see Part II), thus the proper and fast numerical treatment of the Hankel integrals is an important goal. The method used here for the fast Hankel transform follows the paper of Johansen and Sørensen [17]. The basic idea of the fast Hankel transform is to reformulate it as a convolution integral with an analytically given kernel which only needed to be calculated once.

The numerical integration of the Hankel transform (B.19) becomes costly, when $r$ becomes large. This behaviour descends from the oscillations of the Bessel-function $J_{n}(r)$ for large $r$ (see left image of Figure C.I).

The first step to efficiently handle the integral numericly is the following transformation.

Theorem C.2.1 (Fast Hankel transform - substitution). With the substitution

$$
x=\log (r), \quad y=-\log (\lambda)
$$

and the definitions

$$
\begin{gathered}
G(x):=e^{x} g\left(e^{x}\right), \quad F(y):=e^{-y} f\left(e^{-y}\right) \\
K_{n}(x):=e^{x} J_{n}\left(e^{x}\right)
\end{gathered}
$$

the Hankel transform can be written as a convolution integral

$$
G(x)=\int_{-\infty}^{\infty} F(y) K_{n}(x-y) \mathrm{d} y .
$$



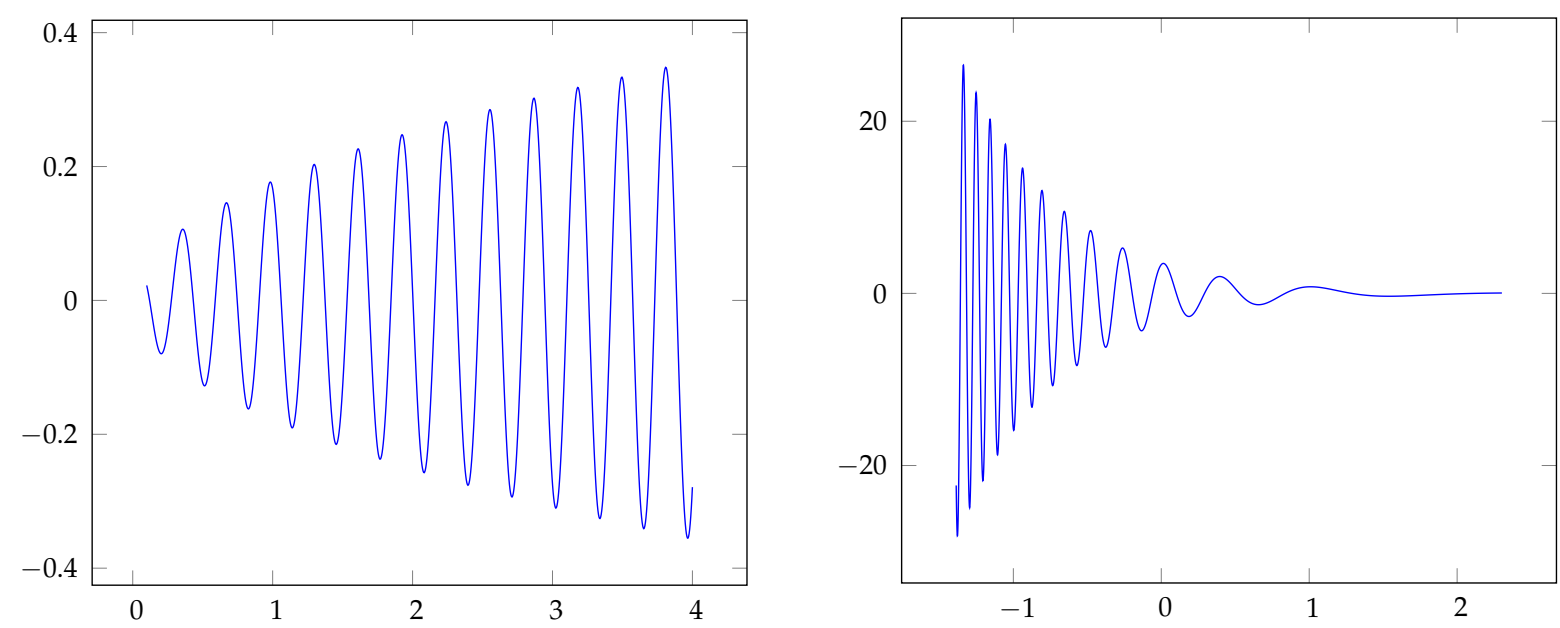

Figure C.1: Example for the kernel of the Hankel transform (left) and the kernel of the substituted Hankel transform (right). Both have order 0.

Proof. With the substitution (C.4) the Hankel transform (B.19) becomes

$$
g\left(e^{x}\right)=\int_{-\infty}^{\infty} f\left(e^{-y}\right) J_{n}\left(e^{x-y}\right) e^{-y}\left(e^{-y}\right) \mathrm{d} y,
$$

and with the definitions (C.5) this leads to

$$
G(x)=\int_{-\infty}^{\infty} F(y) e^{x-y} J_{n}\left(e^{x-y}\right) \mathrm{d} y .
$$

Finally, with (C.6) this transforms to the convolution integral

$$
G(x)=\int_{-\infty}^{\infty} F(y) K_{n}(x-y) \mathrm{d} y
$$

which proves the theorem.

The now changed behaviour of the substituted hankel transform can be seen in the right image of Figure C.1. Now justify the chosen substitution through examining its properties.

Theorem C.2.2 (Fast Hankel transform - substitution properties). The Kernel of the substitution has the property that

$$
\left|\hat{K}_{n}(s)\right|=1, \quad n>1, \quad \Im(s)=0 .
$$

This ensures the existence of the inverse transform and the symmetry of the Hankel transform is preserved. F and $G$ have the same spectra

$$
|\hat{G}(s)|=|\hat{F}(s)|
$$

and the noise in $F$ is neither amplified nor diminished by the convolution with $\mathrm{K}$. 
Proof. Taking the Fourier transform

$$
\hat{K}_{n}(s)=\frac{1}{2 \pi} \int_{-\infty}^{\infty} K_{n}(x) e^{-i x s} \mathrm{~d} x
$$

and using the convolution theorem leads to

$$
\hat{G}(s)=\hat{F}(s) \cdot \hat{K}_{n}(s) .
$$

Using the explicit expression of $K_{n}(x)$ from (C.6) in (C.10), substituting back and using the integral 11.4.16 from [1] leads to

$$
\hat{K}_{n}(s)=\int_{0}^{\infty} J_{n}(t) t^{-i s} \mathrm{~d} t=2^{-i s} \frac{\Gamma((n+1-i s) / 2)}{\Gamma((n+1+i s) / 2)} .
$$

Convergence conditions in (C.12) are given by

$$
\Re(-i s) \leq \frac{1}{2}, \quad \Re(n-i s)>-1
$$

which for real $s$ and $n$ obviously is fulfilled for all $n>-1$. Since the Gamma function fulfils the Schwarz reflection principle ([1, 6.1.23])

$$
\Gamma(\bar{z})=\bar{\Gamma}(z)
$$

the following property holds true for real $s$ and $n>1$

$$
\left|\hat{K}_{n}(s)\right|=1
$$

which implies that the inverse Fourier transform exists. This also means that $F$ and $G$ have the same spectra

$$
|\hat{G}(s)|=|\hat{F}(s)|\left|\hat{K}_{n}(s)\right|=|\hat{F}(s)|
$$

Hence, the noise in $F$ is neither amplified nor diminished by the convolution with $K_{n}$. This completes the proof of the theorem.

Remark (Justification of the substitution). The Theorem is valid for all $n>-1$ and thus covers a wide range of problems. Further, since the noise in $F$ is neither amplified nor diminished by the convolution with $K_{n}$, the numerical realization of the Hankel transform can be simplified in some important steps. It is possible to get an analytic representation of $K_{n}$, which then only needs to be calculated once.

The kernel $K_{n}(r)$ still oscillates exponentially with $r$ getting larger and has a significant distribution to the integral and as such cannot be neglected. In the case of slowly changing $F(y)$, i.e. it is nearly constant over a period of the fast oscillating function $K_{n}(r)$, then the integral over a period of $K_{n}(r)$ is nearly zero. The main idea of the fast Hankel transform is to low pass filter the function $K_{n}(r)$ which reduces the fast oscillating behaviour and leaves the underlying low oscillations of significance for the integral. 
The numerical calculation of the convolution integral (C.7) in a fast way is the goal of this paragraph. First, create equidistant points $y_{p}:=p h, p \in \mathbb{Z}$ with stepsize $h \in \mathbb{R}$. Approximate the function $F$ with

$$
\tilde{F}(y):=\sum_{p=-\infty}^{\infty} F\left(y_{p}\right) P\left(y-y_{p}\right),
$$

with interpolating function $P$ which has the property

$$
P(p h)=\left\{\begin{array}{ll}
1 & , p=0 \\
0 & , p \neq 0
\end{array},\right.
$$

thus the supporting points are reproduced. Then the approximation of the function $G(x)$ from (C.7) becomes

$$
\tilde{G}(x):=\int_{-\infty}^{\infty} \tilde{F}(y) K_{n}(x-y) \mathrm{d} y=\sum_{p=-\infty}^{\infty} F\left(y_{p}\right) \tilde{K}_{n}\left(x-y_{p}\right)
$$

where

$$
\tilde{K}_{n}(x):=\int_{-\infty}^{\infty} P(y) K_{n}(x-y) \mathrm{d} y .
$$

Then, evaluating on equidistant points $x_{m}:=m h, m \in \mathbb{Z}$ leads to

$$
\tilde{G}\left(x_{m}\right)=\sum_{p=-\infty}^{\infty} F\left(y_{p}\right) \tilde{K}_{n}\left(x_{m}-y_{p}\right)=\sum_{p=-\infty}^{\infty} F\left(y_{p}\right) \tilde{K}_{n}((m-p) h) .
$$

The function $\tilde{K}_{n}\left(x_{m}-y_{p}\right)=\tilde{K}_{n}((m-p) h)$ now only depends on $h$ and the order of the Bessel function and hence it is sufficient to calculate $\tilde{K}_{n}$ once for all differences $m-p$ and later interpolate for arbitrary points if necessary.

Further, it is possible to give an analytic expression for $\tilde{K}_{n}$, which is covered by the following Theorem.

Theorem C.2.3 (Analytic form of $\tilde{K}_{n}$ ). Choosing the interpolating function as a smoothed sinc-function

$$
P(y):=\frac{\sin \left(s_{N} y\right)}{s_{N} y} \cdot a \frac{s_{N} y}{\sinh \left(a s_{N} y\right)}=a \frac{\sin \left(s_{N} y\right)}{\sinh \left(a s_{N} y\right)}
$$

where $s_{N}:=\pi / h$ is the Nyquist frequency and $a:=h /(d \pi)$ a smoothness factor with $d \in \mathbb{N} \cup\{0\}$. For $a=0$ this smoothed function is the sinc-function. Then (C.14) is given analytical as

$$
\begin{aligned}
\tilde{K}_{n}(x)= & c \cdot e^{(n+1) x} \sum_{k=0}^{\infty} \frac{(-1)^{k}}{4^{k} \Gamma(k+1) \Gamma(n+1+k)} e^{2 k x} \\
& -2 a \cos \left(x s_{N}\right) e^{x s_{N} / d} \Im\left\{\sum_{l=0}^{\infty} e^{x 2 l s_{N} / d} \hat{K}_{n}\left(s_{N}-i(2 l+1) a s_{N}\right)\right\},
\end{aligned}
$$




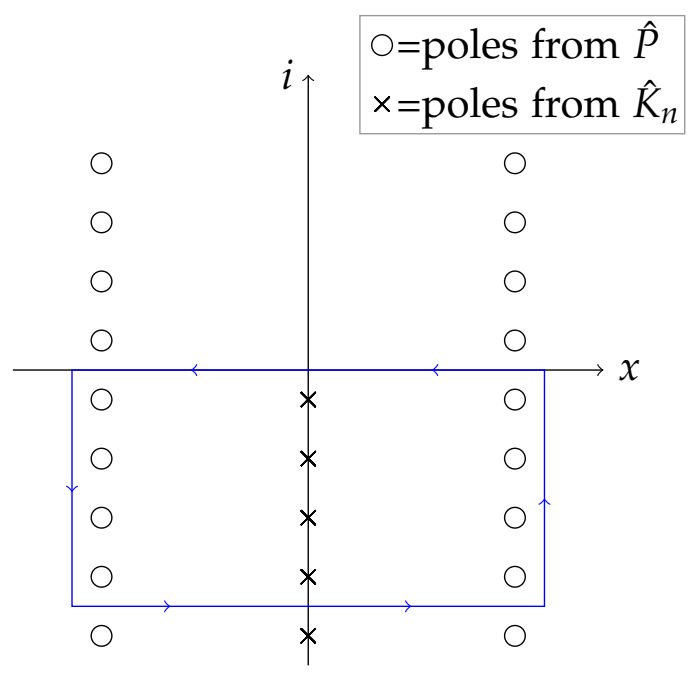

Figure C.2: Integration Path $C$ for residue Theorem and the poles of the functions $\hat{P}$ and $\hat{K}_{n}$. Note: the integration path is extended to $-\infty$ in the imaginary axis.

where $c$ is an oscillating exponential factor defined as

$$
c=i h\left(\frac{1-e^{s_{N} \pi d / 2}}{2+(-1)^{d(n+1)}}\right) 2^{-(n+1)}
$$

Proof. First, write (C.14) as a Fourier integral through the convolution theorem

$$
\tilde{K}_{n}(x)=\frac{1}{2 \pi} \int_{-\infty}^{\infty} \hat{P}(s) \hat{K}_{n}(s) e^{i s x} \mathrm{~d} s .
$$

The goal is to use the residue Theorem for the integration. For this the residues of both $\hat{P}$ and $\hat{K}_{n}$ has to be determined and the integration in the chosen path $C$ in the complex plane has to vanish besides the integration on the real axis. The integration path $C$ is chosen as shown in Figure C.2.

The function $\hat{K}_{n}(s)$ (C.12) is an analytic function in the complex plane except in the poles

$$
p_{k, \hat{K}_{n}}:=-i(n+1+2 k), \quad k \in \mathbb{N}_{0}
$$

with the residues

$$
R_{k, \hat{K}_{n}}:=(-1)^{k} \frac{2^{-(n+1+2 k)}}{\Gamma(k+1) \Gamma(n+1+k)}, \quad k \in \mathbb{N}_{0} .
$$

In the lower halfplane the function $\hat{K}_{n}(s)$ decays faster than any exponential function for $\Im(s) \rightarrow-\infty$ and as such the integration over the path vanishes besides the integration on the real axis.

Further, the interpolating function $P$ has the Fourier transform

$$
\hat{P}(s)=\frac{h}{2}\left(\tanh \left(\frac{\pi\left(s+s_{N}\right)}{2 a s_{N}}\right)-\tanh \left(\frac{\pi\left(s-s_{N}\right)}{2 a s_{N}}\right)\right) .
$$



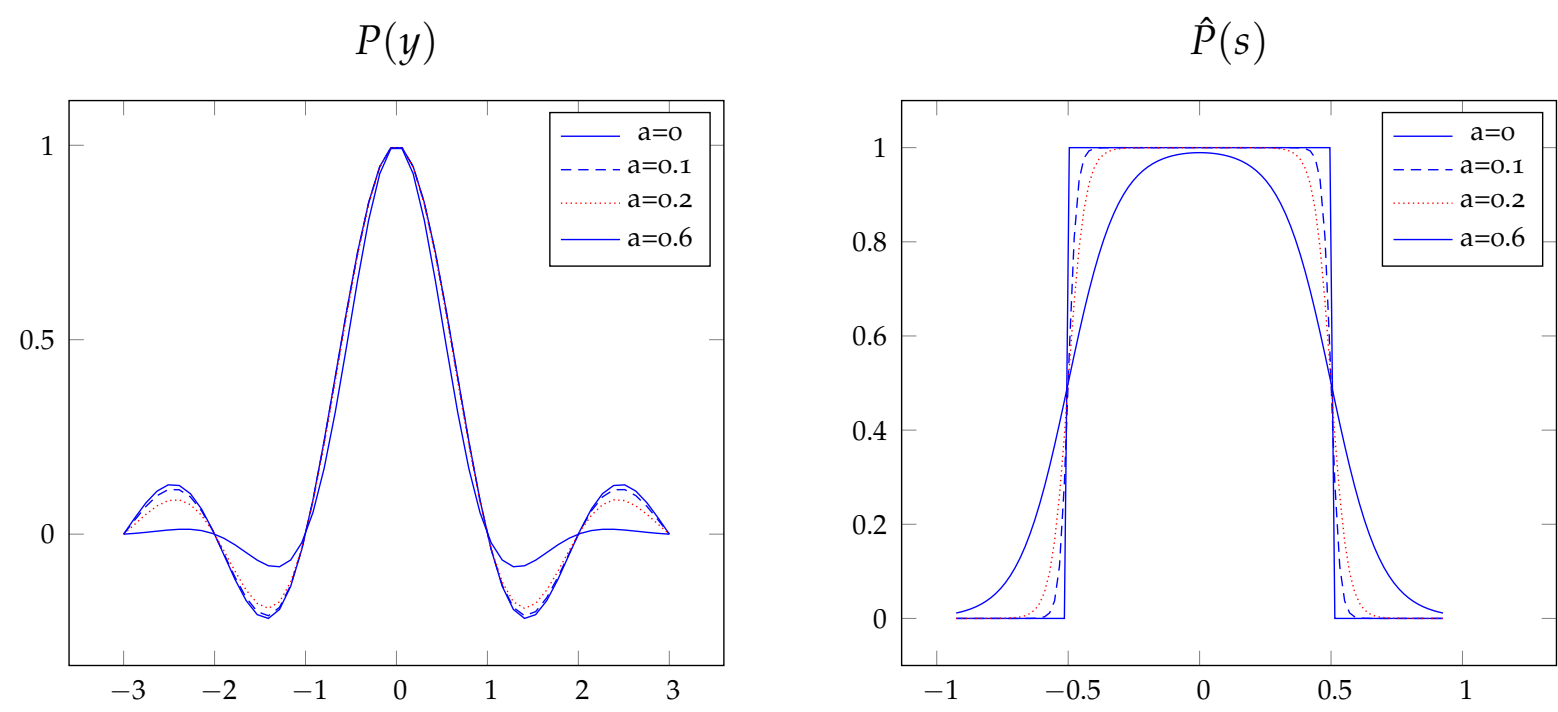

Figure C.3: Example for the dependence of $P(y)$ (left) and $\hat{P}(s)$ (right) on the smoothness parameter $a$.

This is shown in Figure C.3 for some values of the smoothness factor $a$. For $a=0$ the function resembles the sinc-function, and with this function the interpolation (C.14) is exact if $\hat{K}_{n}(s)$ is bandlimited and $|s|>s_{N}$ due to the Shannon sampling Theorem. On the downside the sinc-function would make the integral numericly more costly because it only decays with $1 /|x|$. Smoothing the sinc-function leads to a faster decay, and thus lesser numerical cost, with only a small decrease of accuracy for the interpolation.

The poles of the function $\hat{P}$ in the complex plane are given as

$$
p_{k, \hat{P}}^{ \pm}:= \pm s_{N}-i(2 k+1) a s_{N}, \quad k \in \mathbb{N}_{0}
$$

In the lower halfplane the function $\hat{P}(s)$ decays faster than any exponential function for $\Im(s) \rightarrow \infty$.

Now, it is possible to evaluate the whole integral in (C.17) with the residue Theorem:

$$
\begin{aligned}
\tilde{K}_{n}(x)= & i \sum_{k=0}^{\infty} \hat{P}\left(p_{k, \hat{K}_{n}}\right) R_{k, \hat{K}_{n}} e^{i x p_{k, \hat{K}_{n}}} \\
& +i \sum_{l=0}^{\infty}\left\{R_{l, \hat{P}}^{+} \hat{K}_{n}\left(p_{l, \hat{P}}^{+}\right) e^{i x p_{l, \hat{P}}^{+}}+R_{l, \hat{P}}^{-} \hat{K}_{n}\left(p_{l, \hat{P}}^{-}\right) e^{i x p_{l, \hat{P}}^{-}}\right\}
\end{aligned}
$$


Inserting all the poles and residues and making minor simplifications leads to

$$
\begin{aligned}
& \tilde{K}_{n}(x)= i \frac{h}{2}\left(\tanh \left(\frac{-\pi d\left(i(n+1)+s_{N}\right)}{2}\right)-\tanh \left(\frac{-\pi d\left(i(n+1)-s_{N}\right)}{2}\right)\right) \\
& \cdot 2^{-(n+1)} e^{(n+1) x} \sum_{k=0}^{\infty} \frac{(-1)^{k}}{4^{k} \Gamma(k+1) \Gamma(n+1+k)} e^{2 k x} \\
&+i a \sum_{l=0}^{\infty} e^{\frac{x(2 l+1) s_{N}}{d}}\left\{\hat{K}_{n}\left(s_{N}-i(2 l+1) a s_{N}\right) e^{i x s_{N}}-\hat{K}_{n}\left(-s_{N}-i(2 l+1) a s_{N}\right) e^{-i x s_{N}}\right\} .
\end{aligned}
$$

Using the exponential representation of the tangens hyperbolicus yields

$$
\begin{aligned}
& \tilde{K}_{n}(x)=i h\left(\frac{1-e^{s_{N} \pi d / 2}}{2+(-1)^{d(n+1)}}\right) 2^{-(n+1)} e^{(n+1) x} \sum_{k=0}^{\infty} \frac{(-1)^{k}}{4^{k} \Gamma(k+1) \Gamma(n+1+k)} e^{2 k x} \\
& +i a \sum_{l=0}^{\infty} e^{\frac{x(2 l+1) s_{N}}{d}}\left\{\hat{K}_{n}\left(s_{N}-i(2 l+1) a s_{N}\right) e^{i x s_{N}}-\hat{K}_{n}\left(-s_{N}-i(2 l+1) a s_{N}\right) e^{-i x s_{N}}\right\}
\end{aligned}
$$

Then, with the identity

$$
\hat{K}_{n}(-s+i t)=\overline{\hat{K}_{n}(s+i t)},
$$

and using the Schwartz reflecting principle in the last sum only real parts remain and thus can be simplified to

$$
\begin{aligned}
\tilde{K}_{n}(x)= & i h\left(\frac{1-e^{s_{N} \pi d / 2}}{2+(-1)^{d(n+1)}}\right) 2^{-(n+1)} e^{(n+1) x} \sum_{k=0}^{\infty} \frac{(-1)^{k}}{4^{k} \Gamma(k+1) \Gamma(n+1+k)} e^{2 k x} \\
& -2 a \cos \left(x s_{N}\right) e^{x s_{N} / d} \Im\left\{\sum_{l=0}^{\infty} e^{x 2 l s_{N} / d} \hat{K}_{n}\left(s_{N}-i(2 l+1) a s_{N}\right)\right\},
\end{aligned}
$$

which completes the proof.

Numericly calculating the $\hat{K}_{n}$ has some difficulties which are in detail analysed in [17]. In this paper of Johansen also the sampling error is determined analytically.

Summarising, the fast Hankel transform speeds up the numerical calculations by a large factor. It beats the fast Fourier transform by approximately a factor of 25. This is accomplished mainly through a low pass filtering of the Kernel function $K_{n}$, which then is given analytically and can be calculated a priori. Further, it can be shown that $\tilde{K}_{n}$ is decaying exponentially and thus the infinite sum in (C.15) can be calculated efficiently (see [17]). 


\section{Bibliography}

[1] M. Abramowitz and I. Stegun. Handbook of Mathematical Functions. Dover Publications, New York, 1965. 142

[2] I. Akduman and R. Potthast. On the reconstruction of buried objects from surface measurements of electromagnetic fields. In Proceedings of the European Symposium on Numerical Methods in Electrodynamics, Toulouse, 2002. 83

[3] T. Angell and A. Kirsch. The conductive boundary condition for Maxwell's equations. Journal on Applied Mathematics, 52(6):1597-1610, Dec 1992. (doi:10.1137/0152092). 87

[4] H. Brakhage and P. Werner. Über das Dirichletsche Aussenraumproblem für die Helmholtzsche Schwingungsgleichung. Arch. Math., 16:325-329, 1965. ISSN 0003-9268. 26

[5] O. P. Bruno and L. A. Kunyansky. A Fast, High-Order Algorithm for the Solution of Surface Scattering Problems: Basic Implementation, Tests, and Applications. Journal of Computational Physics, 169(1):80-110, 2001. ISSN 0021-9991. (doi:10.1006/jcph.2001.6714) . 105

[6] O. P. Bruno and L. A. Kunyansky. Surface scattering in three dimensions: an accelerated high-order solver. R. Soc. Lond. Proc. Ser. A Math. Phys. Eng. Sci., 457 (2016):2921-2934, 2001. ISSN 1364-5021. (doi:10.1098/rspa.2001.0882) . 105

[7] D. Colton and A. Kirsch. A simple method for solving inverse scattering problems in the resonance region. Inverse Problems, 12(4):383-393, 1996. ISSN 02665611. (doi:10.1088/0266-5611/12/4/003) . 2

[8] D. Colton and R. Kress. Inverse acoustic and electromagnetic scattering theory, volume 93 of Applied Mathematical Sciences. Springer-Verlag, Berlin, second edition, 1998. ISBN 3-540-62838-X. 6, 17, 19, 20, 21, 24, 25, 26, 28, 32, 34, 35, 66, 75, 81, $82,91,102,105,132,139$

[9] D. L. Colton and R. Kress. Integral equation methods in scattering theory. Pure and Applied Mathematics. John Wiley \& Sons Inc., New York, 1983. ISBN o-47186420-X. A Wiley-Interscience Publication. 26, 75, 130

[10] F. Delbary, K. Erhard, R. Kress, R. Potthast, and J. Schulz. Inverse electromagnetic scattering in a two-layered medium with an application to mine detection. Inverse Problems, 24(1):015002 (18pp), 2008. (doi:10.1088/02665611/24/1/o15002). URL http://stacks.iop.org/0266-5611/24/015002.5, 66 
[11] K. Erhard and R. Potthast. A numerical study of the probe method. SIAM J. Sci. Comput., 28(5):1597-1612 (electronic), 2006. ISSN 1064-8275. (doi:10.1137/040607149) . 49

[12] E. Fredholm. Sur une classe d'équations fonctionnelles. Acta Mathematica, 27: 365-390, 1903. 123, 124

[13] M. Ganesh and I. Graham. A high-order algorithm for obstacle scattering in three dimensions. Journal of Computational Physics, 198(1):211-242, 2004. ISSN 0021-9991. (doi:10.1016/j.jcp.2004.01.007) • 105

[14] W. Gröbner and N. Hofreiter. Integraltafel - Bestimmte Integrale, volume 2. Springer Verlag, Wien - New York, 1966. 136

[15] J. Hadamard. Lectures on Cauchy's problem in linear partial differential equations. Dover Publications, New York, 1953. 125

[16] M. Ikehata. Enclosing a polygonal cavity in a two-dimensional bounded domain from Cauchy data. Inverse Problems, 15(5):1231-1241, 1999. ISSN 0266-5611. 2

[17] H. K. Johansen and K. Sørensen. Fast Hankel transforms. Geophysical prospecting, 27:876-901, 1979. 107, 140, 146

[18] A. Kirsch. Characterization of the shape of a scattering obstacle using the spectral data of the far field operator. Inverse Problems, 14(6):1489-1512, 1998. ISSN 0266-5611. (doi:10.1088/0266-5611/14/6/oo9) . 2

[19] A. Kirsch and R. Kress. On an integral equation of the first kind in inverse acoustic scattering. In Inverse problems (Oberwolfach, 1986), volume 77 of Internat. Schriftenreihe Numer. Math., pages 93-102. Birkhäuser, Basel, 1986. 3, 6, 34

[20] A. Kirsch and R. Kress. A numerical method for an inverse scattering problem. In Inverse and ill-posed problems (Sankt Wolfgang, 1986), volume 4 of Notes Rep. Math. Sci. Engrg., pages 279-289. Academic Press, Boston, MA, 1987. 3, 6, 34

[21] A. Kirsch and R. Kress. An optimization method in inverse acoustic scattering (invited contribution). In Boundary elements IX, Vol. 3 (Stuttgart, 1987), pages 3-18. Comput. Mech., Southampton, 1987. 3, 6, 34

[22] R. Kress. Electromagnetic waves scattering: Specific theoretical tools. In Pike and Sabatier, editors, Scattering, pages 175-190. Academic Press, London, 2001. 86,92

[23] R. Kress. Linear integral equations, volume 82 of Applied Mathematical Sciences. Springer-Verlag, New York, second edition, 1999. ISBN o-387-98700-2. 19, 47, $123,124,126,128,130$

[24] S. Kusiak and J. Sylvester. The scattering support. Communications on pure and applied mathematics, LVI(11):1525-1548, 2003. 31 
[25] J. C. Lagarias, J. A. Reeds, M. H. Wright, and P. E. Wright. Convergence properties of the Nelder-Mead simplex method in low dimensions. SIAM Journal on Optimization, 9(1):112-147, 1998. ISSN 1052-6234. (doi:10.1137/S1052623496303470) . URL http://link.aip.org/link/?SJE/9/112/1. 7, 96

[26] R. M. Lewis, V. Torczon, and M. W. Trosset. Direct search methods: then and now. J. Comput. Appl. Math., 124(1-2):191-207, 2000. ISSN 0377-0427. (doi:10.1016/So377-0427(00)00423-4) . Numerical analysis 2000, Vol. IV, Optimization and nonlinear equations. 97

[27] D. R. Luke and R. Potthast. The no response test - a sampling method for inverse scattering problems. SIAM Journal on Applied Mathematics, 63(4):1292-1312, 2003. ISSN oo36-1399. (doi:10.1137/Soo36139902406887) . 2, 49

[28] P. Monk. Finite Element Methods for Maxwell's Equations. Clarendon Press / Oxford University Press, 2003. ISBN 978-0198508885. 66

[29] C. Müller. Foundations of the Mathematical Theory of Eletromagnetic Waves, volume 155 of Die Grundlehren der mathematischen Wissenschaften in Einzeldarstellungen. Springer-Verlag, 1969. 87

[30] J. A. Nelder and R. Mead. A simplex method for function minimization. Computer Journal, 7:308-313, 1965. 96

[31] E. J. Nyström. Über die praktische Auflösung von Integralgleichungen mit Anwendungen auf Randwertaufgaben. Acta Mathematica, 54:185-2-4, 1930. 139

[32] O. Panich. On the question of the solvability of the exterior boundary-value problems for the wave equation and Maxwell's equations. Usp. Mat Nauk, 2oA: 221-226, 1965. Russian. 27

[33] M. Petry. Über die Streuung zeitharmonischer Wellen im geschichteten Raum. PhD thesis, Georg-August University of Göttingen, 1993. 66, 90, 107

[34] R. Potthast. Stability estimates and reconstructions in inverse acoustic scattering using singular sources. J. Comput. Appl. Math., 114(2):247-274, 2000. ISSN 03770427. (doi:10.1016/So377-0427(99)00201-o) . 2, 4, 6, 43

[35] R. Potthast. Point sources and multipoles in inverse scattering theory, volume 427 of Chapman $\mathcal{E}$ Hall/CRC Research Notes in Mathematics. Chapman \& Hall/CRC, Boca Raton, FL, 2001. ISBN 1-58488-252-2. 3, 4, 6, 19, 25, 30, 38, 39, 43, 49

[36] R. Potthast. A survey on sampling and probe methods for inverse problems. Inverse Problems, 22(2):R1-R47, 2006. ISSN 0266-5611. (doi:10.1088/o266$5611 / 22 / 2 / \operatorname{RoI})$. 2, 49

[37] R. Potthast. A point source method for inverse acoustic and electromagnetic obstacle scattering problems. IMA J. Appl. Math., 61(2):119-140, 1998. (doi:10.1093/imamat/61.2.119) . 6, 43 
[38] R. Potthast. Point sources and multipoles in inverse scattering theory. Habilitation thesis, Göttingen, 1999. 6, 43

[39] R. Potthast. A set-handling approach for the no-response test and related methods. Mathematics and Computers in Simulation, 66:281-295, 2004. ISSN 0378-4754. (doi:10.1016/j.matcom.2004.02.012) · 40

[40] R. Potthast and J. Schulz. A multiwave range test for obstacle reconstructions with unknown physical properties. Journal of Computational and Applied Mathematics, 205:53-71, 2007. ISSN 0377-0427. (doi:10.1016/j.cam.2006.04.040) . 2

[41] R. Potthast, J. Sylvester, and S. Kusiak. A 'range test' for determining scatterers with unknown physical properties. Inverse Problems, 19(3):533-547, 2003. ISSN 0266-5611. (doi:10.1088/0266-5611/19/3/304) . 1, 29, 31, 33, 37, 99

[42] F. Riesz. Über lineare Funktionalgleichungen. Acta Mathematica, 41:71-98, 1918. 123

[43] A. Sommerfeld. Partial Differential Equations in Physics. Academic Press, New York, 1949. 16, 134

[44] A. N. Tikhonov and V. Y. Arsenin. Solutions of ill-posed problem. Winston, New York, 1977. 127

[45] V. Torczon. On the convergence of pattern search algorithms. SIAM J. Optim., 7 (1):1-25, 1997. ISSN 1052-6234. (doi:10.1137/S1052623493250780) . 97

[46] G. N. Watson. Theory of Bessel functions. Cambridge University Press, 1922. 135

[47] L. Wienert. Die numerische Approximation von Randintegraloperatoren fuer die Helmholtzgleichung im R3. PhD thesis, Georg-August University of Göttingen, 1990. 105

[48] P. Wilde. Über das Transmissionsproblem bei der vektoriellen Helmholtzgleichung. $\mathrm{PhD}$ thesis, Georg-August University of Göttingen, 1985. 90

[49] M. H. Wright. Direct search methods: once scorned, now respectable. In $\mathrm{Nu}$ merical analysis 1995 (Dundee, 1995), volume 344 of Pitman Res. Notes Math. Ser., pages 191-208. Longman, Harlow, 1996. 97 


\section{List of Figures}

1.1 Setup and idea of the range test (a) and the multiwave range test (b). . . 3

1.2 Common setting for mine detection with a handheld detector(C)2003 Foerster Instruments Incorporated) . . . . . . . . . . . . . . . 4

2.1 Hermann Ludwig Ferdinand von Helmholtz (1821 - 1894) and Leonhard Euler (1707 [Pleaseinsertintopreamble] 1783) . . . . . . . . . . 14

2.2 Arnold Johannes Wilhelm Sommerfeld (1868 - 1951)(public domain). . . . 16

2.3 Franz Rellich $(1906-1955)$ (unknown) . . . . . . . . . . . . . . . 18

2.4 Real part of the fundamental solution for the Helmholtz equation in 2D. 19

3.1 Setup of acoustic scattering. . . . . . . . . . . . . 24

4.1 Setup and idea of the range test. . . . . . . . . . . 30

4.2 Setup and idea of the potential method a) and the modified potential

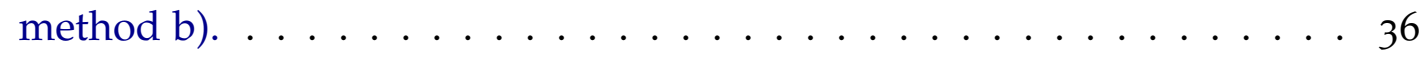

4.3 Idea and setup of the multiwave range test. . . . . . . . . . . . . . . . . . . . . 39

4.4 Configuration and area of small $\left\|\psi_{x}\right\|$ (below). . . . . . . . . . . . . . . 41

4.5 Configuration and area of small $\left\|\psi_{x}\right\|$ (right). . . . . . . . . . . . . . . 42

4.6 Idea and setup of the singular sources method. . . . . . . . . . . 43

4.7 The Diagram shows the relation between the one-wave range test, the potential method, the multiwave range test and the singular sources

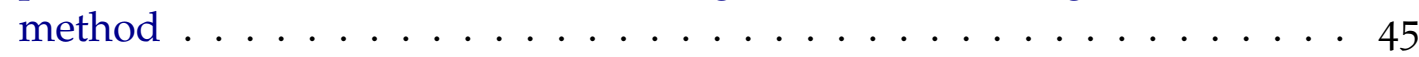

5.1 Illustration of the total field from calculating the direct scattering problem for a boat-like scatterer. . . . . . . . . . . . . . . . 48

5.2 Reconstruction of the shape with the range test. . . . . . . . 50

5.3 Field reconstructions with the modified method of Kirsch-Kress in the case of Dirichlet boundary conditions. . . . . . . . . . . . 51

5.4 Field reconstructions with the modified method of Kirsch-Kress in the case of Neumann boundary conditions. . . . . . . . . . . . . . . 52

5.5 Reconstruction of the shape with the multiwave range test. . . . . . . 53

5.6 Reconstruction of the shape with the singular sources method. . . . . 53

6.1 Common setting for mine detection with a handheld detector(@)2003 Foerster Instruments Incorporated) . . . . . . . . . . . . . . . . . 60

6.2 Scheme where the different Green's tensors are valid. . . . . . . . . . . 65

6.3 Wire loop of source $\Gamma_{s}$ and receiver $\Gamma_{r} \ldots \ldots \ldots \ldots \ldots$

8.1 Simplices after an iteration step. . . . . . . . . . . . . . 98 


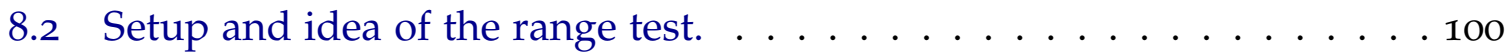

9.1 Triangulation of a sphere and ellipse in $(0,0,-0.1) \mathrm{m} . \ldots \ldots . . \ldots 106$

9.2 Setting for the simulation. . . . . . . . . . . . . 108

9.3 Simulated induced voltages of a homogeneous background medium with a sphere and an ellipse. . . . . . . . . . . . . 108

9.4 Relative error regarding number of triangles in a double logarithmic plot. . . . . . . . . . . . . . . . . . . 110

9.5 Setting for the reconstructions with the Nelder-Mead simplex method. . III

9.6 Shape reconstructions in a homogenous background with a perfect

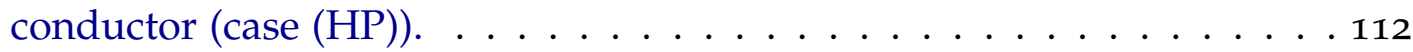

9.7 Shape reconstructions in a layered background with a perfect conduc-

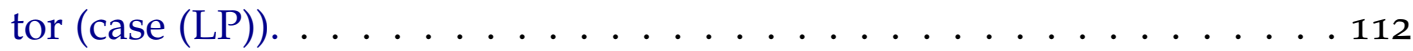

9.8 Shape reconstructions in a homogenous background with a conducting obstacle (case $(\mathrm{HT}))$. . . . . . . . . . . . . . . . 113

9.9 Shape reconstructions in a layered background medium with a conducting obstacle (case (LT)) . . . . . . . . . . . . . . . 113

9.10 Setting for the reconstructions with the range test. . . . . . . . . . 114

9.11 Reconstructions with the range test for an ellipse in a slice horizontally through the center of the obstacle. . . . . . . . . . . . 115

9.12 Reconstructions with the range test for an ellipse. . . . . . . . . . . 116

9.13 Reconstructions with the range test for a sphere in a slice horizontally through the center of the obstacle. . . . . . . . . . . . 117

9.14 Reconstructions with the range test for a sphere. . . . . . . . . . 117

A.1 Frigyes Riesz $(1880-1956)$ (public domain). . . . . . . . . . . . . . . 123

A.2 Jacques Solomon Hadamard $(1865$ - 1963) (public domain). . . . . . . . . 125

A.3 Andrey Nikolayevich Tychonoff (1906 - 1993)(C)2006 Russian Academy of Sciences). . . . . . . . . . . . . . . . . . . . . 128

B.1 Original essay on Green's theorem by George Green (1793 - 1841) (public domain). . . . . . . . . . . . . . . . . . 130

B.2 Bessel functions of order $0 . \ldots \ldots \ldots \ldots \ldots \ldots$

C.1 Integration Path $C$ for residue Theorem and the kernel of the substituted Hankel transform. . . . . . . . . . . . . . . . . . . . 141

C.2 Integration Path $C$ for residue Theorem. . . . . . . . . . . . . . . 144

C.3 Example for the dependence of $P(y)$ and $\hat{P}(s)$ on the smoothness parameter $a \ldots \ldots \ldots \ldots \ldots \ldots \ldots \ldots$ 


\section{List of Tables}

6.1 Fundamental physical constants. . . . . . . . . . . . . . . 78

6.2 Example for some material constants. . . . . . . . . . . . 78

9.1 Convergence rates. . . . . . . . . . . . . . . . 109 
Nomenclature

154 


\section{Nomenclature}

$C^{k} \quad$ domain of class $C^{k}, 122$

$C^{k}(D) \quad$ function which is $k$-times continuously differentiable on the domain $D, 122$

$C^{l} \quad$ l-times continuous differentiable functions, 8

$C^{l}(D) \quad$ space of $l$-times continously differentiable functions on $D, 8$

$C^{l, \alpha}(D) \quad$ space of $l$-times Hölder continously differentiable functions on $D, 8$

$C_{t, d}(\partial D) \quad$ space of continuous tangential fields posessing a surface Divergence, 74

$C_{t, d}^{0, \alpha}(\partial D) \quad$ space of Hölder continuous tangential fields posessing a surface Divergence, 74

$C_{t}(\partial D) \quad$ space of continuous tangential fields, 74

$C_{t}^{0, \alpha}(\partial D) \quad$ space of Hölder continuous tangential fields, 74

$D^{\alpha} f(x) \quad$ partial derivatives of $f(x)$ with multi-index $\alpha, 8$

$D_{0} \quad$ free space without obstacle, 59

$D_{1} \quad$ upper halfspace, 59

$D_{2} \quad$ lower halfspace without obstacle, 59

$D_{3}, D \quad$ obstacle, 59

$G_{E, j} \quad$ Electric Green's tensor. $j=0$ : free space, $j=3$ inside obstacle, $j=1,2$ : the two layers., 65

$G_{H, j} \quad$ Magnetic Green's tensor. $j=0$ : free space, $j=3$ inside obstacle, $j=1$, 2: the two layers., 65

$H_{n}^{(0,1)} \quad$ spherical Hankel functions of first and second kind with order $n, 133$

$J_{n} \quad$ spherical Bessel functions of order $n, 133$

K double layer operator, 20

$K^{\prime} \quad$ adjoint of the double layer operator, 20 


\begin{tabular}{|c|c|}
\hline$M_{j}$ & magnetic dipole operator, 75 \\
\hline$N_{j}$ & electric dipole operator, 75 \\
\hline$N_{n}$ & spherical Neumann functions of order $n, 133$ \\
\hline$R_{\alpha}$ & regularised inverse, 127 \\
\hline$S$ & single layer operator, 19 \\
\hline$S_{0}$ & Single layer operator in the limit $\kappa \rightarrow 0,27$ \\
\hline$T$ & normal derivative of double layer operator, 20 \\
\hline$Y_{n}$ & spherical harmonics of order $n, 133$ \\
\hline$\Gamma_{r}$ & curve of the receiver wire loop, 77 \\
\hline$\Gamma_{S}$ & curve of the source wire loop, 76 \\
\hline $\mathbb{N}_{0}$ & Natural numbers $\mathbb{N}$ with $0 ., 8$ \\
\hline S & unit sphere, 17 \\
\hline$\Theta$ & configuration, 40 \\
\hline$|a|$ & euclidean norm, 8 \\
\hline$\alpha$ & regularisation parameter, 127 \\
\hline$a \odot b$ & $\boldsymbol{a} \odot \boldsymbol{b}:=\left(a_{1} b_{1}, \ldots, a_{n} b_{n}\right), 107$ \\
\hline $\mathrm{cS}_{\kappa} \operatorname{supp} u_{\infty}$ & convex scattering support of the far field $u^{\infty} ., 31$ \\
\hline$\epsilon_{j}$ & electric permittivity, 59 \\
\hline$\frac{\partial u_{ \pm}}{\partial v}$ & jump relation of the single layer potential, 21 \\
\hline$\gamma_{m}$ & constant for far field patterns, 17 \\
\hline$\hat{x}$ & normalised $x$, i.e. directions., 16 \\
\hline$\kappa$ & wave number defined as $\kappa:=\frac{\omega}{c}, 15$ \\
\hline$\kappa$ & wave number, 60 \\
\hline$\mu_{j}$ & magnetic permeability, 59 \\
\hline$\|\cdot\|_{\infty}$ & supremum norm, 8 \\
\hline$v$ & normal vector, 16 \\
\hline$\omega$ & frequency of electromagnetic wave, 59 \\
\hline
\end{tabular}




$\begin{array}{ll}\omega & \text { frequency, 15 } \\ \bar{a} & \text { conjugate complex of } a, 8 \\ \sigma_{j} & \text { electric conductivity, } 59 \\ \Phi & \text { fundamental solution of the Helmholtz equation, 19 } \\ a \cdot b & \text { bilinear scalar product, } 8 \\ c_{S} & \text { Speed of sound, 15 } \\ u^{\infty} & \text { far field pattern, 17 } \\ v_{ \pm} & \text {jump relation of the double layer potential, 21 } \\ (\text { HP) } & \text { homogeneous background medium with perfectly conducting obsta- } \\ \text { cle, } 79 & \text { homogeneous background medium with conducting obstacle, } 79 \\ (\text { LP) } & \text { layered background medium with perfectly conducting obstacle, } 79 \\ \text { (LT) } & \text { layered background medium with conducting obstacle, } 79\end{array}$


Nomenclature

158 


\section{Index}

A

acoustic scattering problem $\ldots \ldots \ldots \ldots 24$

adiabatic hypothesis .............. 14

\section{B}

Bessel differential equation, sph. . . . 132

Bessel functions, spherical ... 68, 133, 134

recurrence relations ........... 134

Biot-Savart's law ................ 76

boundary condition

perfect conductor $\ldots \ldots \ldots \ldots \ldots \ldots 62$

transmission ................62 62

Dirichlet ................... 16

Neumann .................... 16

sound-hard ................... 16

sound-soft .................. 16

bounded operators ............. 121

C

classes of domains .............. 122

compact operators ............... 121

configuration $\ldots . \ldots \ldots \ldots \ldots \ldots \ldots, 40$

convex scattering support ....... 31, 101

dipole

electric $\ldots \ldots \ldots \ldots \ldots \ldots \ldots \ldots, 76$

magnetic .................. 76

direct acoustic scattering

Dirichlet .................... 25

Neumann .................... 27

direct electromagnetic scattering ..... 79

homogeneous, conductor (HT) ...88

homogeneous, perfect conductor (HP) 80

layered, conductor (LT) ........ 91

layered, perfect conductor (LP) . . 8 83

Dirichlet boundary condition ........ 16

double layer operator $\ldots \ldots \ldots \ldots \ldots .20$

adjoint $\ldots \ldots \ldots \ldots \ldots \ldots \ldots \ldots, 20$ normal derivative $\ldots . \ldots \ldots \ldots \ldots 20$

double layer potential ............ 20

jump relation $\ldots \ldots \ldots \ldots \ldots \ldots .21$

\section{E}

electric conductivity $\ldots \ldots \ldots \ldots \ldots \ldots 59$

electric dipole $\ldots \ldots \ldots \ldots \ldots \ldots \ldots \ldots, 76$

electric dipole operator $\ldots \ldots \ldots \ldots \ldots .75$

electric permittivity $\ldots \ldots \ldots \ldots \ldots \ldots . \ldots 59$

electromagnetic inverse problem .... 95

equation of continuity ............ 13

Equivalence of radiation conditions .. 63

Euler's equation ................. 13

Euler, Leonhard ................. 14

existence

acoustic Dirichlet ............. 26

acoustic Neumann ............. 27

electromagnetic (HP) ......... 81

electromagnetic $(\mathrm{HT}) \ldots \ldots \ldots . \ldots 90$

electromagnetic (LP) ........... 86

electromagnetic (LT) ........... 94

\section{$\mathbf{F}$}

far field pattern $\ldots \ldots \ldots \ldots \ldots \ldots \ldots \quad 17$

Faraday's law ................... 77

Fast Hankel transform ............ 140

Foerster MINEX 2FD 4.500 ........ 107

Fourier transform .............. 134

Fredholm alternative ......... 124, 125

Fredholm, Erik Ivar ............. 123

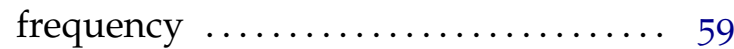

fundamental solution, acoustic ...... 19

G

Gamma function $\ldots \ldots \ldots \ldots \ldots \ldots, 142$

Green's

first theorem ................ 129

formula ................... 130

second theorem ............... 129

Green's tensor $\ldots \ldots \ldots \ldots \ldots \ldots \ldots \ldots, 72$ 
free space $\ldots . \ldots \ldots \ldots \ldots \ldots \ldots 65$

layered media ................ 66

Green, George ................. 129

H

Hadamard, Jacques Solomon ....... 125

handheld mine detection $\ldots \ldots \ldots \ldots .76$

Hankel function ............. 19, 133

Hankel transform ............... 134

Helmholtz equation ......... 13, 15, 23

Helmholtz representation ......... 130

Helmholtz, Hermann von .......... 13

Huygens principle ................ 19

\section{I}

ill-posed problem ............... 126

integral equation

solvability ................. 124

integral operators

domain $\ldots \ldots \ldots \ldots \ldots \ldots \ldots \ldots, 122$

surface $\ldots \ldots \ldots \ldots \ldots \ldots \ldots \ldots, 122$

\section{J}

jump relation

double layer potential .......... 21

single layer potential ........... 21

vector surface potential $\ldots . \ldots \ldots .74$

$\mathrm{K}$

Kirsch-Kress method, modified ...... 36

\section{M}

magnetic dipole $\ldots \ldots \ldots \ldots \ldots \ldots \ldots, 76$

magnetic dipole operator ........... 75

magnetic permeability ............. 59

Maxwell equations ................ 59

time-harmonic ........... 60, 67

mixed reciprocity relation $\ldots \ldots \ldots \ldots .25$

multiwave range test $\ldots \ldots \ldots \ldots \ldots \ldots, 42$

inverse problem $\ldots \ldots \ldots \ldots \ldots \ldots 3^{8}$

\section{$\mathrm{N}$}

Nelder-Mead algorithm ........... 99

Neumann boundary condition ....... 16

Neumann functions, spherical ...... 133

normal vector $\ldots \ldots \ldots \ldots \ldots \ldots \ldots .16$

Nyquist frequency .............. 143

Nyström interpolation formula ..... 140

Nyström method ............. 105, 140
Nyström, Evert J.

\section{0}

operator equation

first kind $\ldots \ldots \ldots \ldots \ldots \ldots \ldots \ldots \ldots$

second kind ................ 123

operators

bounded ..................... 121

compact $\ldots \ldots \ldots \ldots \ldots \ldots \ldots \ldots$. 121

integral .................... 122

integral, surface $\ldots \ldots \ldots \ldots \ldots \ldots 122$

\section{$\mathbf{P}$}

perfect conductor boundary condition 62 plane wave

acoustic ................... 23

electromagnetic .............. 114

potential method

inverse problem ................ 35

potential method, modified ......... 36

\section{$\mathbf{R}$}

radiation condition

Silver-Müller .................. 63

Sommerfeld .................. 16

range test, acoustic $\ldots \ldots \ldots \ldots \ldots \ldots . \ldots 33$

inverse problem $\ldots \ldots \ldots \ldots \ldots \ldots 29$

multiwave $\ldots \ldots \ldots \ldots \ldots \ldots \ldots, 42$

range test, electromagnetic ........ 103

inverse problem .............. 99

reciprocity relation

far field $\ldots \ldots \ldots \ldots \ldots \ldots \ldots \ldots 24$

mixed ...................... 25

regular strategy $\ldots \ldots \ldots \ldots \ldots \ldots . \quad 127$

regularisation

parameter ................. 127

scheme ................... 127

Rellich's lemma

acoustic ................... 17

acoustic, extended ............. 25

electromagnetic, extended ....... 64

Rellich, Franz ................ 17

Riesz, Frigyes ............... 123

S

scattering support $\ldots \ldots \ldots \ldots \ldots .6$ 31, 101

Schwartz reflection principle ...... 142

Silver-Müller radiation condition .... . 63

single layer operator .............. 19 
single layer potential $\ldots \ldots \ldots \ldots \ldots \ldots \quad 19$

jump relation $\ldots \ldots \ldots \ldots \ldots \ldots .21$

singular sources method

inverse problem ............... 43

singular sources method, alternative .. 44

Sommerfeld identity/integral ...... 135

Sommerfeld radiation condition ...... 16

Sommerfeld, Arnold .............. 16

speed of sound $\ldots \ldots \ldots \ldots \ldots \ldots \ldots \ldots$

spherical harmonics .............. 133

state equation $\ldots \ldots \ldots \ldots \ldots \ldots \ldots . . \ldots \ldots$

Stratton-Chu formulas ........... 132

surface divergence $\ldots \ldots \ldots \ldots \ldots \ldots . \ldots 73$

surface gradient $\ldots \ldots \ldots \ldots \ldots \ldots \ldots, 73$

\section{T}

Tikhonov regularisation $\ldots \ldots \ldots \ldots \ldots, 128$

Tikhonov, Andrey Nikolayevich ..... 127

time harmonic fields $\ldots . \ldots \ldots \ldots .60$

time-harmonic Maxwell equations .... 60

total field ...................... 23

transmission boundary condition .... 62

transmission conditions

air-soil

U

uniqueness

acoustic Dirichlet ............. 25

acoustic Neumann ............. 27

electromagnetic (HP) .......... 80

electromagnetic (HT) ......... 88

electromagnetic (LP) ..........8 83

electromagnetic (LT) .......... 91

V

Vector Helmholtz equation ......... 61

vector surface potential ............ 74

jump relation $\ldots \ldots \ldots \ldots \ldots \ldots .74$

W

wave equation $\ldots \ldots \ldots \ldots \ldots \ldots \ldots \ldots$. 15

wave number $\ldots \ldots \ldots \ldots \ldots \ldots \ldots .15$

electromagnetic ..............6 60

weakly singular

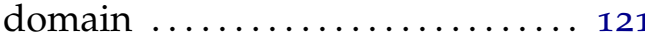

surface $\ldots \ldots \ldots \ldots \ldots \ldots \ldots \ldots . \ldots \ldots$

well-posed problem .............. 126

equations of the first kind ...... 126

wire loop $\ldots \ldots \ldots \ldots \ldots \ldots \ldots \ldots, 77$ 


\section{Curriculum Vitae}

\section{Personal Data}

Name:

Jochen Schulz

Date of birth:

17.05.1976

Place of birth:

Hannover

Citizenship:

Germany

Family status:

Unmarried

Parents:

Karla Schulz, née Kerner and Hermann Schulz

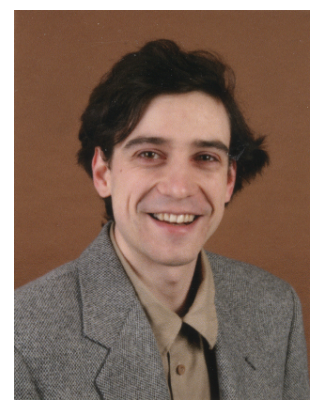

\section{Education:}

$1982-1986$

$1986-1988$

1988 - 1995

May 1995
Primary school Wettbergen, Hannover.

Middle School Martensplatz, Hannover.

Secondary school Humboldtschule Hannover.

University-entrance diploma (Abitur).

\section{Educational background:}

Oct. 1995 - Feb. 2001

Jan. 2000 - Jan. 2001

$1998-1999$

Apr. 2000 - Oct. 2001

since October 2001

Jan. 2002 - Dec. 2002

Oct. 2002 - Feb. 2003

Jul. 2003 - Aug. 2003

Jul. 2005 - Sep. 2005

Oct. 2005 - Jun. 2007
Study of physics at the Georg-August university of Göttingen. Diploma thesis with the titel: "Evolution von Galaxien mit unterschiedlichen Bulge- und Scheiben-Anteilen im Vergleich mit Beobachtungen".

System-administrator of a heterogeneous network in the institute of biochemistry II.

Software-developer and system-administrator for the institute of marketing and trade and the company eResult. Development and support of an online-panel.

Ph.D. studies in the institute for numerical and applied mathematics, university of Göttingen.

Research assistant for a project regarding EIT on trees in the university of applied sciences Hildesheim/Holzminden/Göttingen. Assistant for the lecture "Mathematics and Scientific Computing for Inverse Problems".

Research project together with SERATEC GmbH regarding parameter identification.

Research project together with the german aerospace center (DLR) regarding optical measurements of surfaces.

Research assistant in the network "Metal detectors for Humanitarian Demining: Development potentials for data analysis and measurement techniques" which was funded by the german federal ministry of education and research (BMBF). 